\title{
Developments and recent advancements in the field of endogenous amino acid selective bond forming reactions for bioconjugation
}

Cite this: Chem Soc Rev. 2015, 44, 5495

Received 19th January 2015

DOI: $10.1039 / \mathrm{c} 5 \mathrm{cs} 00048 \mathrm{c}$

www.rsc.org/chemsocrev

\author{
Oleksandr Koniev ${ }^{\mathrm{ab}}$ and Alain Wagner*a
}

Bioconjugation methodologies have proven to play a central enabling role in the recent development of biotherapeutics and chemical biology approaches. Recent endeavours in these fields shed light on unprecedented chemical challenges to attain bioselectivity, biocompatibility, and biostability required by modern applications. In this review the current developments in various techniques of selective bond forming reactions of proteins and peptides were highlighted. The utility of each endogenous amino acid-selective conjugation methodology in the fields of biology and protein science has been surveyed with emphasis on the most relevant among reported transformations; selectivity and practical use have been discussed.

\section{Contents}

\section{Introduction \\ 1 In-chain conjugation \\ 1.1 Lysine \\ 1.1.1 Isocyanates and isothiocyanates \\ 1.1.2 Activated esters \\ 1.1.3 Reductive amination of aldehydes \\ 1.1.4 Sulfonyl halides and sulfonates \\ 1.1.5 Fluorobenzenes \\ 1.1.6 Imidoesters \\ 1.1.7 Miscellaneous amine-selective reagents \\ 1.2 Serine and Threonine \\ 1.3 Cysteine \\ 1.3.1 $\alpha$-Halocarbonyls \\ 1.3.2 Maleimides \\ 1.3.3 Vinyl sulfones \\ 1.3.4 Thiol-ene coupling \\ 1.3.5 Thiol-yne coupling \\ 1.3.6 Disulfide reaction \\ 1.3.7 Disulfide rebridging \\ 1.3.8 Transforming to dehydroalanine \\ 1.3.9 Miscellaneous thiol-selective reagents \\ 1.4 \\ 1.4.1 Malondialdehydes \\ 1.4.2 Metallocarbenoids}

${ }^{a}$ Laboratory of Functional Chemo-Systems (UMR 7199), Labex Medalis, University of Strasbourg, 74 Route du Rhin, 67401 Illkirch-Graffenstaden, France. E-mail: alwag@unistra.fr

${ }^{b}$ Syndivia SAS, 4 rue Boussingault, 67000 Strasbourg, France
$1.5 \quad$ Histidine
1.5.1 Epoxides
1.5.2 Complexes with transition metals
1.5.3 Michael addition
1.5.4 Miscellaneous histidine-selective reagents
1.6 Tyrosine
1.6.1 O-Derivatisation
1.6.2 O-Oxidatiove coupling
1.6.3 Diazonium reagents
1.6.4 Mannich-type reaction
1.6.5 Dicarboxylates and dicarboxamides
1.7 Arginine
1.8 Aspartic and glutamic acids
1.9 Methionine
$2 \quad N$-terminal conjugation
$2.1 \quad \alpha$-Amino groups
2.1.1 Classical approaches
2.1.2 Ketene-mediated conjugation
2.1.3 Transamination
2.1.4 2-Pyridinecarboxyaldehydes (2PCA)
2.2 Serine and Threonine
2.2.1 $O \rightarrow \mathrm{N}$ shift of oxazolidines
2.2.2 Periodate oxidation
2.2.3 Phosphate-assisted ligation
2.2.4 Indirect approaches
2.3 Cysteine
2.3.1 Native Chemical Ligation
2.3.2 Thiazolidine formation
2.3.3 2-Cyanobenzothiazoles (CBT)
2.4 Tryptophan
2.4.1 Sulfenylation-coupling 
2.4.2 Pictet-Spengler reaction

2.4.3 $\mathrm{N}$-Acyl tryptophan isopeptides

$2.5 \quad$ Histidine

2.6 Selenocysteine

2.7 Proline

$3 \quad C$-terminal conjugation

4 Sequence-selective approaches

5 Proximity-driven modifications

Conclusions

\section{Introduction}

Bioconjugation is a set of techniques allowing site-specific creation of a covalent link between a biomolecule and an exogenous moiety that endow it with desirable properties. This novel hybrid having the combined properties of its individual components can serve, for instance, as a more stable and efficient therapeutic, ${ }^{1-4}$ an assembly for studying proteins in the biological context, ${ }^{5-11}$ a new protein-based material, ${ }^{12-17}$ a microarray, ${ }^{18,19}$ a biological material, ${ }^{20-24}$ a tool for immobilisation, ${ }^{25}$ and for elucidation of the structure ${ }^{26,27}$ of proteins.

A large number of reactions exist to modify proteins. ${ }^{28}$ However, site-specific conjugation continues to attract considerable research efforts to develop new methodologies that match continuously increasing requirements of modern applications in terms of selectivity, stability, mildness, and preserving biomolecule integrity. For the purpose of this overview, the focus will remain on recent developments in bond-forming approaches in bioconjugation of native amino acid residues. Among about 20 different amino acids involved in protein composition, only a smaller number comprises appropriate targets for practical bioconjugation methods. In fact, only one-third of all amino acid residues represent chemical targets for the vast majority of bond-forming approaches.

The bioconjugation methodology of choice is selected according to the intrinsic reactivity of the targeted amino acid residue

(acidity/basicity, electrophilicity/nucleophilicity, oxido-reductive characteristics) and its specific special environment (in-chain, $\mathrm{N}$-terminal, C-terminal, location in a specific sequence, accessibility, etc.). In this review we will thus present the known bioconjugation strategies in regard to these parameters ranked in a descending order of frequency they are reported in literature.

\section{In-chain conjugation}

\subsection{Lysine}

The use of chemical groups that react with primary amines is one of the oldest and most versatile techniques for protein conjugation. Virtually all proteins contain primary amino groups in their structure. They can be divided into two groups: the $\alpha$-amino group situated in the $\mathrm{N}$-terminus of most polypeptide chains and $\varepsilon$-amino groups of lysine residue (Lys, K). Because these amino groups possess $\mathrm{p} K_{\mathrm{a}}$ values of about 8 and 10 (for $\alpha$ - and $\varepsilon$-amines respectively), in a vast majority of cases they are protonated at physiological $\mathrm{pH}$ and, therefore, occur predominantly on the solvent-exposed outside surfaces of protein tertiary structures. As a result, they become easily accessible to conjugation reagents introduced into the aqueous media.

Deprotonated primary amines are the most nucleophilic among the available functional groups present in a typical protein. However, protonation drastically decreases their reactivity. As a consequence, despite the generally higher intrinsic nucleophilicity of Lys $\varepsilon$-amino groups, they require higher $\mathrm{pH}$ values to be uncovered by deprotonation, which allows distinguishing $\alpha$ - and $\varepsilon$-amino groups by adjusting the $\mathrm{pH}$. That is to say, at the higher $\mathrm{pH}$ level, when both types of primary amines are deprotonated, Lys side chain amino groups are generally more reactive towards electrophiles, while at the lower $\mathrm{pH}$ it is the opposite because of their prior protonation (Fig. 1). At the acidic $\mathrm{pH}$ all amines are protonated and possess no significant nucleophilicity compared to other side chains present in proteins.

Dr Oleksandr (Sasha) Koniev received an $\mathrm{MSc}$ in molecular chemistry from the University of Toulouse (France) in 2010. He subsequently joined the group of Dr Alain Wagner for his graduate studies, where he developed novel bioselective ligation reactions and obtained his PhD from the University of Strasbourg in 2014. The same year, Dr Koniev cofounded and became CEO of Syndivia, a biotechnology company that provides advanced bioconjugation techniques for the development of antibody-drug conjugates. His research interests focus on designing new bioconjugation approaches allowing preparation of biological materials with enhanced performance and their application in anticancer therapies.

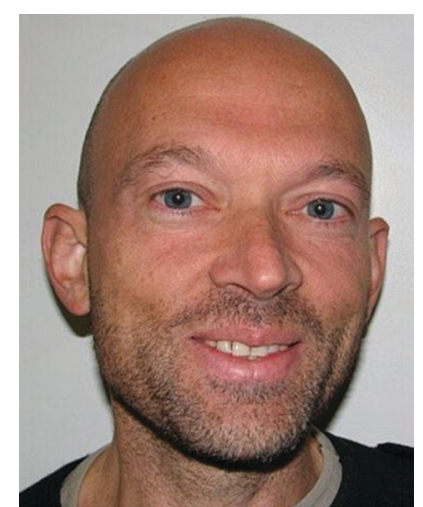

Alain Wagner
Dr Alain Wagner received his $\mathrm{PhD}$ in organic chemistry from Université Louis Pasteur (1991, Strasbourg, France), followed by a post-doctoral fellowship with Prof. Peter Schultz at Affymax Research Institute (1991-1993, Palo Alto, USA). He joined CNRS in 1994 to develop projects involving chemistry and biology. Alain Wagner co-founded a number of successful start-up companies: Novalix Pharma (2002), Phytodia (2007), eNovalys (2009), and most recently Syndivia (2014). Returning to the CNRS (2008), he took the lead of the Laboratory of Functional Chemo-Systems. Dr Wagner has published more than 120 articles in peer-reviewed journals and he is an inventor of 17 patents. 


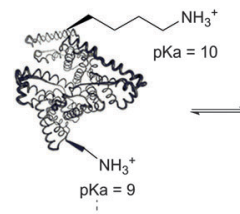

6

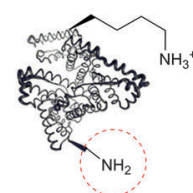

8

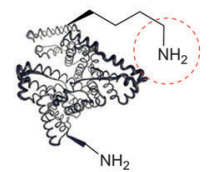

$10 \mathrm{pH}$

Fig. 1 Deprotonation of different types of amino groups present in protein structure (more nucleophilic amine is encircled in red). Lysine $\varepsilon$-amino groups are more nucleophilic, but also more difficult to deprotonate. Generally, a pH of 8.5-9.5 is optimal for modifying lysine residues, while near neutral $\mathrm{pH}$ favours selective modification of $\mathrm{N}$-termini.

In particular, free (non-disulfide-bonded) Cys residues are much stronger nucleophiles and, if accessible, will readily be modified by most amine-reactive reagents.

It is also to be mentioned that like any other parameters, nucleophilicity and basicity, as well as solvent exposure and accessibility of a particular amino group, are influenced by the microenvironment and can vary substantially, regarding the substrate. For instance, Westheimer and Schmidt have found the actual $\mathrm{p} K_{\mathrm{a}}$ of the amino group situated in the active site of acetoacetate decarboxylase to be 5.9 , which is $4 \mathrm{p} K_{\mathrm{a}}$ units less than that of an "ordinary" $\varepsilon$-amino group of lysine. ${ }^{29}$

Depending on reaction conditions, selective modification of either N-termini (see Section 2) or Lys $\varepsilon$-amino groups can be achieved by using various chemical reagents. They generally belong to one of the following classes (in the order of relevancy): activated esters (fluorophenyl esters, NHS ( $N$-hydroxysuccinimides), ${ }^{30}$ sulfo-NHS, acyl azides), isothiocyanates, isocyanates, ${ }^{31}$ aldehydes, anhydrides, sulfonyl chlorides, carbonates, fluorobenzenes, epoxides and imidoesters. Among this vast variety of reactive functions, NHS esters (and their more soluble sulfo-NHS analogues) and imidoesters represent the most popular amine-specific functional groups that are incorporated into commercially available reagents for protein conjugation and labelling. ${ }^{28}$

Despite their name, amine-reactive reagents are not always entirely selective for amines. Firstly, as already mentioned before, they will react with any other stronger nucleophile, if the latter is present and accessible on a protein surface. Particularly, it concerns cysteine, tyrosine, serine and threonine side chains. Secondly, depletion of these highly activated reagents by hydrolysis is inevitable in aqueous solution. The rate of both side-reactions depends on the particular substrate, the conjugation partner, $\mathrm{pH}$, temperature, and buffer composition. Evidently, buffers that contain free amines, such as TRIS (tris(hydroxymethyl)aminomethane), must be avoided when using any amine-reactive probes, since the rate of the reaction with buffer would greatly exceed that with protein amino groups.

1.1.1 Isocyanates and isothiocyanates. Amines undergo a reaction with isocyanates to readily form stable ureas. However, because of their susceptibility to deterioration during storage, ${ }^{32}$ isocyanates are much more difficult to manipulate with and thus are not as well commercially accessible as corresponding isothiocyanates. They can though be easily prepared prior to use from more stable acyl azides by Curtius rearrangement.

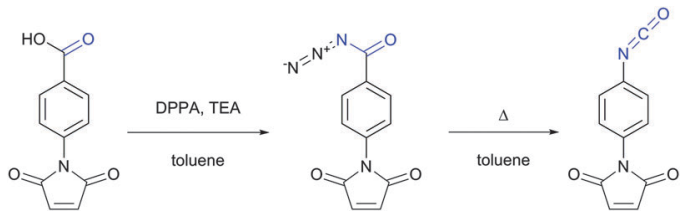

Fig. 2 Synthesis of the $p$-maleimidophenyl isocyanate crosslinker via Curtius rearrangement proposed by Palumbo and associates. ${ }^{33}$

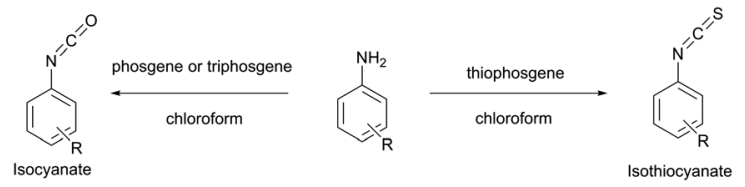

Fig. 3 Synthesis of isothiocyanates and isocyanates from the corresponding aromatic amines. ${ }^{36}$

Using this approach, for instance, Palumbo and colleagues have elaborated the synthesis of a heterobifunctional amine-thiol crosslinker containing an isocyanate group on one end and a thiol reactive maleimide group on the other end (Fig. 2). ${ }^{33}$

Several early studies were devoted to the elaboration of isocyanate conjugation methodology, ${ }^{34,35}$ but proven to be especially laborious and complicated mainly due to the high reactivity and low stability of isocyanates. Therefore they are of deferred interest today, being completely displaced by isothiocyanate-mediated approaches. Both isothiocyanates and isocyanates can be obtained from the corresponding aromatic amines upon reaction with thiophosgene and phosgene respectively (Fig. 3). ${ }^{36}$

Isothiocyanate-based selective amino group modification was first reported in 1937 by Todrick and Walker, ${ }^{37}$ who found that the reaction of allyl isothiocyanate with cysteine in alkaline medium results selectively in thiourea - the product of amine addition to isothiocyanate. In 1950, exploiting the selectivity of amino-terminal labelling of the peptide with phenylisothiocyanate, Edman has developed a method for peptide sequencing that has changed cardinally the protein science and is known today as Edman degradation. ${ }^{38}$ Only 30 years later, Podhradský et al. have examined the reaction of isothiocyanates on complex substrates and demonstrated that the addition of the thiol and phenolate functions of cysteine and tyrosine residues is always prevalent, and that only at $\mathrm{pH}>5$ amino groups start to manifest themselves in the reaction. ${ }^{39}$ While thiol and alcohol additions result in reversible reactions to give dithiocarbamates and $O$-thiocarbamates respectively, amines add themselves irreversibly, thus shifting the reaction equilibrium towards thioureas (Fig. 4). One should however keep in mind that, despite the reversibility of the addition of thiols and alcohols to isocyanates, they can enhance the kinetics of their hydrolysis to unreactive amines or ureas and therefore significantly decrease the yield of the conjugation. Moderately reactive but quite stable in water and most solvents, isothiocyanates represent thus an appropriate alternative to the unstable isocyanates. As a consequence, they are much more popular in bioconjugation.

Ever since the introduction of fluorescent isothiocyanate dyes as more stable analogues of corresponding isocyanates for fluorescent labelling of antibodies by Riggs et al. ${ }^{40}$ in 1958, 


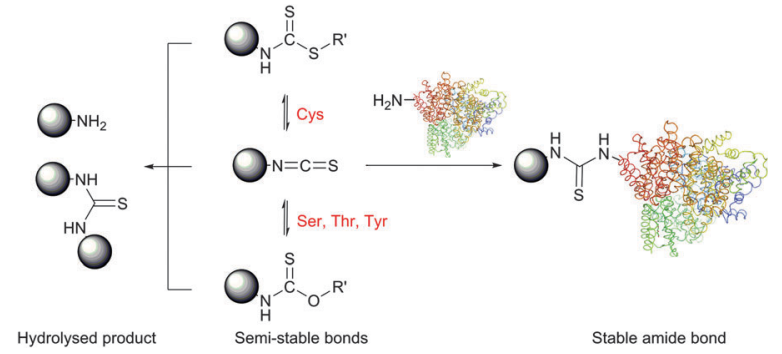

Fig. 4 Reaction of isothiocyanates with nucleophilic amino acid residues present in proteins. Only the reaction of lysine and $\mathrm{N}$-terminal residues (considerable at $\mathrm{pH}>5$ ) results irreversibly in obtaining the thiourea. Although the reactions of thiol and alcohol groups with isothiocyanates are reversible, they can largely accelerate the rate of isothiocyanate hydrolysis.

they have found widespread use in research laboratories and proved to be an effective means for tagging proteins at specific sites. $^{41}$

Fluorescein isothiocyanate (FITC) is arguably one of the most commonly used fluorescent derivatisation reagents for proteins. For instance, it was reported by Tuls et ll. $^{42}$ that cytochrome P-450 can be selectively labelled by FITC with 75\% yield of a single-labelled LYS-338 conjugate in TRIS (particularly inappropriate buffer for amine-reactive reagents though) at $\mathrm{pH} 8.0$ and $0{ }^{\circ} \mathrm{C}$. Burtnick ${ }^{43}$ has described selective labelling of one out of 34 lysine residues of actin in borate buffer with 35-fold excess of the reagent at $\mathrm{pH}$ 8.5. Such a high level of selectivity towards the LYS-61 residue over other 33 lysine residues present in proteins (Fig. 5, shown in red) remains unclear, but it is hypothesised to be due to an anomalously low $\mathrm{p} K_{\mathrm{a}}$ value thereof. Following reports of Miki and collaborators ${ }^{44,45}$ further confirmed the selectivity of this labelling, yet without any explanation of such specificity. Bellelli et al. ${ }^{46}$ were able to covalently label ricin ( $\mathrm{pH} \mathrm{8.1,} 6^{\circ} \mathrm{C}$ for $4 \mathrm{~h}$ ). In fact, the targets of the isothiocyanate-mediated labelling of proteins elaborated over the last 60 years are even difficult to enumerate. It was proven to be effective in diverse applications such as tagging of antibodies (usually in carbonate-bicarbonate buffer, $\mathrm{pH} 9$ ), ${ }^{4-53}$ bleaching-based measurement of membrane protein diffusion of FITC-labelled cells ( $\mathrm{pH} 9.5,24{ }^{\circ} \mathrm{C}$ ), ${ }^{54}$ surface topography of the Escherichia coli ribosomal subunit, ${ }^{55} \alpha$-actinin distribution

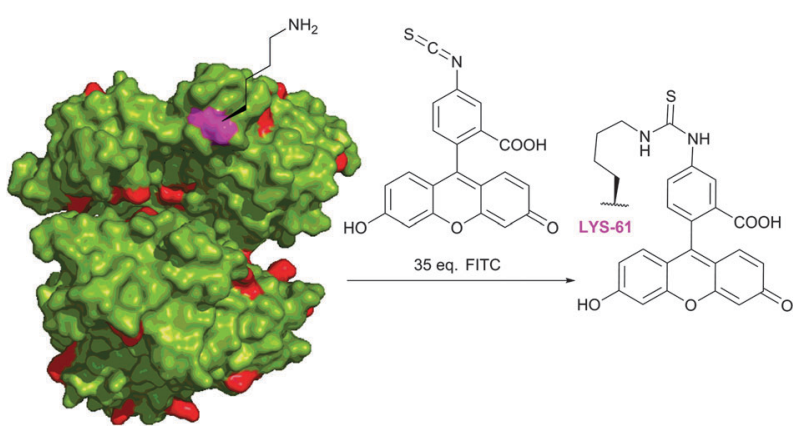

Fig. 5 Selective fluorescent labelling of the Lys-61 residue (shown in magenta) of rabbit skeletal muscle G-actin (pdb: 2VYP) reported by Burtnick. $^{43}$ in living and fixed fibroblasts, ${ }^{56}$ characterisation of a proton pump on lysosomes, ${ }^{57}$ and hematopoietic stem cells. ${ }^{58}$

The most stunning examples include ${ }^{125}$ I labelling by means of isothiocyanates, elaborated by Shapiro and colleagues ${ }^{59}$ for regional differentiation of the sperm surface (TRIS, $\mathrm{pH}$ 7.7, $12{ }^{\circ} \mathrm{C}$ for $30 \mathrm{~min}$ ), and the application of a similar methodology by Schirrmacher et al. ${ }^{60}$ for ${ }^{18} \mathrm{~F}$ radioactive labelling of RSA, apotransferrin and bovine IgG (pH 9.0, room temperature for 10-20 min). Conjugation of antibodies with chelating agents for further radiometal labelling of antibodies has been described by several groups ${ }^{61-63}$ and is based on the use of phenylisothiocyanate-containing probes. Brechbiel et al. ${ }^{64}$ went even further by combining the chelating functionality with the biotin fragment in a scaffold of trifunctional conjugation reagents. The preparation of silica nanoparticles coated with isothiocyanate groups and their use in apoptosis detection has recently been elaborated. ${ }^{65}$

The classical protocol of isothiocyanate labelling involves the use of 5-10 equivalents at a slightly basic $\mathrm{pH}$ in the range of 9.0-9.5. ${ }^{66,67}$ Resulting thioureas are reasonably stable in aqueous medium and provide a suitable degree of conjugation. ${ }^{68}$ For example, Sandmaier and colleagues ${ }^{69}$ have recently demonstrated that radiolabelling of the anticanine CD45 antibody using isocyanate and isothiocyanate provides a more specific delivery to the targeted CD45-expressing cells than a method exploiting thiol-maleimide conjugation (see Section 1.3.2). However, it has been shown by Banks and Paquette ${ }^{70}$ that, compared to NHS ester based methodology, antibody conjugates prepared with isothiocyanates are less hydrolytically stable and deteriorate over time. Moreover, the reaction of NHS esters for amine labelling was found to be faster, to give more stable conjugates for both model amino acids and proteins, and to proceed readily at lower $\mathrm{pH}$, compared to isothiocyanates. Consequently, NHS esters are preferable to isothiocyanates in many respects for synthesizing bioconjugates.

1.1.2 Activated esters. Because of the poor leaving ability of the alkoxy groups, alkyl esters of carboxylic acids are inert to amines in aqueous media. ${ }^{71}$ However, their substitution by good leaving groups activates the carbonyl and renders it susceptible to nucleophilic attack. It is worth remarking that not only does such activation increase the reactivity of these reagents towards free amino groups, but also often augment their tendency to degrade in the presence of water. ${ }^{72}$ Although many activating moieties have appeared over years, only a limited number of them are of significant importance in bioconjugation today. For instance, formerly of significant importance, especially in the field of peptide synthesis, activated phenyl esters are almost of no use today in bioconjugation because of their lower kinetics and lower stability compared to succimidyl esters. ${ }^{73-75}$ However, they continue to reappear in certain studies. ${ }^{23}$

$\mathrm{N}$-Hydroxysuccinimide (NHS) activated esters were introduced in 1963 by Anderson et al. as a better alternative to phenyl esters in forming the peptide bond. ${ }^{30,76,77}$ Possessing high selectivity towards aliphatic amines, NHS esters are today 


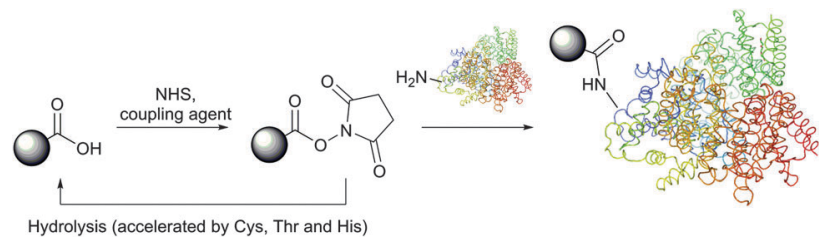

Fig. 6 Preparation of activated NHS-esters and their reaction with nucleophilic amino acid residues present in proteins. Similarly to isothiocyanates, the reversible reactions of thiol and alcohol groups with NHS-esters largely increase the rate of hydrolysis thereof.

considered among the most powerful protein-modification reagents. Although several studies drew attention to a certain reactivity of NHS-activated esters with tyrosine,${ }^{78-83}$ histidine, ${ }^{84}$ serine and threonine (especially when situated in certain locations, see Section 1.2), ${ }^{85-90}$ these side reactions possess largely decreased rates compared to the reaction with free amines and do not generally hinder the amine-selective derivatisation. High concentrations of nucleophilic thiols should however be avoided because, similarly to isothiocyanates, they may increase the rate of probe degradation by forming more easily hydrolysable intermediates (Fig. 6).

The optimum $\mathrm{pH}$ for NHS-mediated labelling in aqueous systems was found to be lower than for other amine-selective reagents and ranges from 7 to 8 units (compared with 9-9.5 for isothiocyanates), which enlarges the prospect of its suitability for modifying alkaline-sensitive proteins. Several elaborated studies of the kinetics, ${ }^{72}$ the stoichiometry, ${ }^{91}$ and the selectivity (in-chain versus $\mathrm{N}$-terminal modification) ${ }^{92}$ of NHS-mediated protein tagging have been recently reported.

Depending on the $\mathrm{pH}$ of the reaction solution and temperature, NHS esters are hydrolysed by water (possessing a half-life of 4-5 hours at $\mathrm{pH} 7,1$ hour at $\mathrm{pH} 8$ and 10 minutes at $\mathrm{pH} 8.6),{ }^{84,93}$ but are stable to storage if kept well desiccated. Virtually any molecule containing an acid functionality, or a moiety which can give an acid, can be transformed into its $N$-hydroxysuccinimide ester. While the activation with NHS generally decreases the watersolubility of the carboxylate molecule, the utilisation of sulfo-NHS ${ }^{94}$ preserves or even increases the water-solubility of the modified molecule by virtue of the charged sulfonate group. The development of new reagents based on NHS chemistry can be sometimes challenging, ${ }^{95}$ but the derivatives are frequently of very important use. $^{96-100}$ Many NHS derivatives for the preparation of affinity reagents, fluorescent probes and cross-coupling reagents are now commercially available, enabling wide access to investigations.

The formed conjugates are linked by means of a very stable aliphatic amide bond with half-lives in the range of 7 years in water. ${ }^{101}$ This excellent stability and biocompatibility of the obtained bonds have provided an exceptional importance of NHS esters in the field of bioconjugation.

NHS ester-mediated covalent conjugation for protein modification has been first accomplished by Becker et al., who studied biotin transport first in yeast ${ }^{74}$ and then applied this technique to the covalent attachment of biotin to bacteriophage $\mathrm{T} 4 .^{102}$ Since then, the field of NHS-mediated conjugation of proteins has been unceasingly expanding its employability in countless applications.
Cross-linking of proteins often implies using NHS-containing homobifunctional or heterobifunctional cross-linking reagents. These were used for elucidation of protein-protein ${ }^{103-107}$ and protein-drug interactions, ${ }^{108}$ protein structural and subunit analysis, ${ }^{26,109}$ create protein complex models, ${ }^{110}$ and preparation of protein conjugates with enzymes, drugs or other macromolecules. ${ }^{111-113}$

Homobifunctional NHS cross-linkers are generally used in reaction procedures to randomly "fix" or polymerize peptides or proteins through their amino groups. Adding such crosslinkers to a cell lysate will result in the random conjugation of interacting proteins, protein subunits, and any other polypeptides whose Lys side chains happen to be in close proximity to each other. This represents a methodology for capturing a "snapshot" of all protein interactions at a certain instant of time. Using this approach, for instance, Sinz and collaborators were able to elucidate binding of calmodulin to mettilin, a polypeptide and principal component of honeybee venom, without chromatographic separation techniques. ${ }^{114}$ Cross-linking of the proteins with a number of different length NHS-homobifunctional cross-linkers, and the following digestion of obtained products with trypsin and analysis by HPLC enabled the possibility of three-dimensional structure modelling of the calmodulin-melittin complex (Fig. 7).

Several applications however require the precision of crosslinking which cannot be provided by homobifunctional crosslinkers. For example, the preparation of an antibody-drug conjugate (ADC) implies selective linking of a cytotoxic payload to each molecule of the antibody without causing any antibodyto-antibody linkages to form. For such application the combination of different selective approaches in one linker is needed.

Therefore, heterobifunctional crosslinkers are designed to possess different reactive groups at either end. These reagents allow for sequential conjugations that diminish undesirable self-conjugation and polymerisation. Sequential procedures involve two-step processes, where heterobifunctional reagents

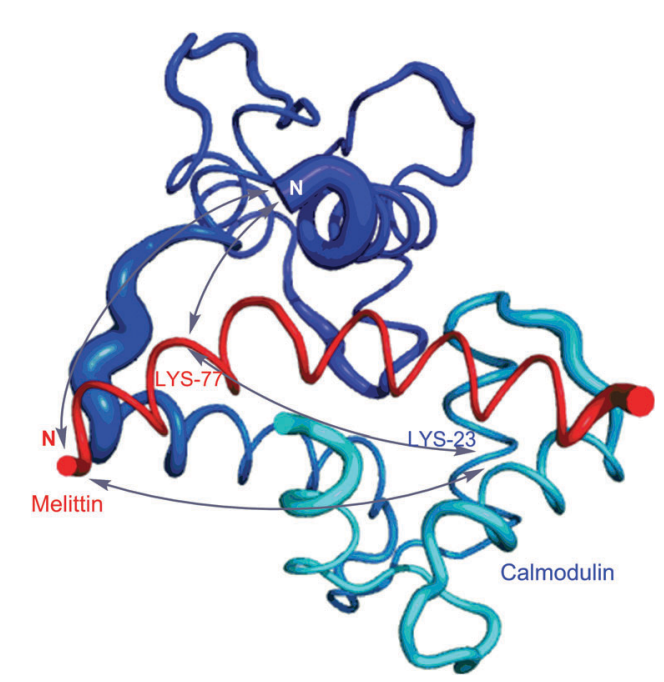

Fig. 7 Mode of binding of melittin in the calmodulin-melittin complex (pdb: $2 M L T$ and 1CDL) calculated from ambiguous distance restraints derived from the cross-linking data by Sinz and associates. ${ }^{114}$ 
(often in excess to ensure high conversion levels) are reacted with one protein using the most labile group of the crosslinker first. After eliminating the excess of the nonreacted crosslinker, the second protein is added to a solution containing modified first protein and another reaction occurs with the second reactive group of the crosslinker. According to the Pierce website (Rockford, IL, USA), the most popular heterobifunctional crosslinkers are those having amine-reactive NHS esters at one end and thiol-reactive maleimides (see Section 1.3.2) at the other end. Because of its less stability in aqueous solution compared to maleimide, the NHS-ester group should usually be reacted first. Takeda and co-workers ${ }^{115}$ used a bifunctional reagent that contained a NHS function and a benzylthioester function to prepare a DNA-protein hybrid. One of the fastest growing fields requiring heterobifunctional crosslinkers today is targeted drug delivery therapies - ADCs. ${ }^{22,116-120}$ They are constituted of three main components: one monoclonal antibody (mAb), targeting specific signs or markers of cancer cells, one cytotoxic agent, and one linker molecule that allows covalent drug binding to the mAb. The composition of trastuzumab emtansine (Kadcyla ${ }^{\mathbb{R}}$, Genentech), an in clinic ADC for treatment of HER2-positive metastatic breast cancer, is depicted in Fig. 8.

The first example of a "cleavable" NHS cross-linking reagent, DSP, was reported by Lomant and Fairbanks ${ }^{93}$ and allowed effecting the reversal of the previously conjugated fragments under mild conditions of disulfide bond reduction. Further advances in the field have resulted in various types of linkers, cleavable under mild nucleophilic conditions (EGS), ${ }^{121}$ at basic $\mathrm{pH}$ (BSOCOES), ${ }^{122}$ in the presence of periodate (DST), ${ }^{123}$ or enzymatically. ${ }^{124}$ These found their applicability for studies in basic and applied research. The reader is directed to a recent review by Leriche $e t$ al. ${ }^{125}$ that provides an overview of chemical functions that can be used as cleavable agents and to a publication by Jin Lee ${ }^{126}$ for an overview of commercially available cross-linking reagents.

Other combinations of functionalities have been studied over the last 20 years and resulted in elaboration of heterotrifunctional ${ }^{127}$ linkers usually combining two bioselective reactive groups and a functionality for anchoring the obtained conjugate (e.g. the biotin moiety).

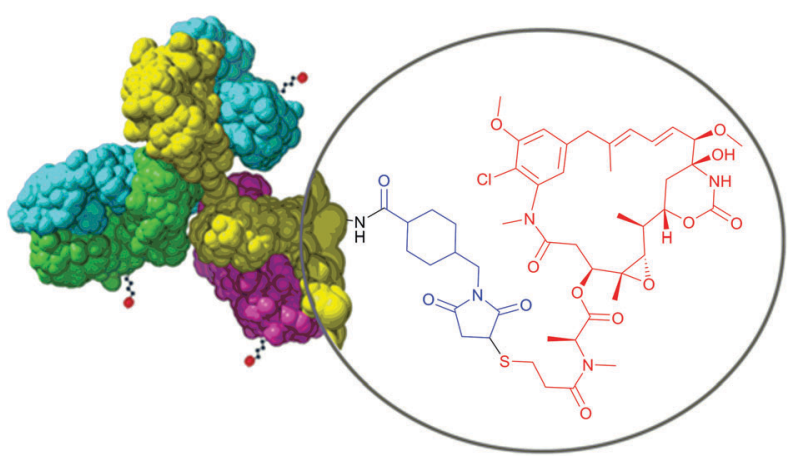

Fig. 8 Structure of an antibody drug conjugate (ADC) Kadcyla ${ }^{\circledR}$. SMCC linker (shown in blue) serves for conjugation of the antibody and cytotoxic payload (Mertansine, shown in red).
Many chemical probes widely used in bioconjugation contain the NHS-fragment in their structure and are designed to react with free amino groups of proteins. For example, biotinylation ${ }^{128-130}$ as well as PEGylation ${ }^{2}$ of proteins are most commonly achieved using NHS-activated probes today. It was recently reported by Anderson and collaborators that biotinylation of antibodies with NHS-biotin and their following adsorption on the surface of nanocrystal quantum-dots (QD) results in obtaining highly efficient QD-antibody conjugates for the detection of protein toxins. ${ }^{131}$ Other types of protein immobilisation on matrices have also been reported. ${ }^{84,132,133}$ The Bolton-Hunter reagent (SHPP), ${ }^{134}$ allowing the conjugation of tyrosine-like residues for increasing the yield of subsequent (radio)iodination, is also based on $N$-hydroxysuccinimide chemistry. ${ }^{135-140}$ Elaborated in 1982 by Ji et $a l .{ }^{141}$ structurally similar SHPP photoactivable heterobifunctional probes for cross-linking experiments have been used in more than 100 studies ever since. The NHS ester-based strategy for isobaric, stable isotope labelling of peptides ${ }^{142-144}$ has recently found more widespread application in proteomic studies with simultaneous developments in enhancing peptide detection by electrospray ionisation mass spectrometry. ${ }^{78,145}$ This list can be continued and arguably utilisation of NHSmediated techniques can be found in all major fields of protein conjugation and represents a gold standard in bioconjugation.

1.1.3 Reductive amination of aldehydes. Aliphatic and aromatic amines react under mild aqueous conditions with aldehyde groups to form an imine (known as a Schiff base). This intermediate can then be selectively reduced by a mild reducing agent, such as sodium cyanoborohydride, ${ }^{146}$ to give a stable alkylamine bond. Although this approach for amine modification is not used in protein conjugations as frequently as the activated ester or isothiocyanate method, it is to be considered as preferable when the molecule to be attached has an aldehyde group (or can be easily converted to an aldehyde) because of its simplicity and mild reaction conditions.

Historically, the conjugation of oligosaccharides to proteins has become the first target for this approach. In 1974, relying on the exceptional ability of the cyanoborohydride anion described three years earlier by Borch ${ }^{146}$ to reduce selectively Schiff bases generated in situ from an amine and an aldehyde, Gray has illustrated the possibility of mild synthesis of carbohydrate coated bovine serum albumin (BSA) and P150 protein (Fig. 9) ${ }^{147}$ However, because of low kinetics of conjugation, only 4 out of 59 BSA lysine residues (presumably those possessing the lowest $\mathrm{p} K_{\mathrm{a}}$ values) were derivatised after 300 hours of reaction.

Reductive amination of proteins proceeds most readily at $\mathrm{pH}$ 6.5-8.5 where the reduction of aldehydes and ketones is negligible, and, if feasible, in an alcoholic solution under dehydrating conditions where the rate-limiting formation of the imine is favoured. According to Allred and colleagues, ${ }^{148}$ the addition of sodium sulfate $(500 \mathrm{mM})$ may largely improve the coupling efficiency in aqueous media.

To date, reductive amination has played a central role in the synthesis of carbohydrate-protein conjugates, ${ }^{20,149-151}$ which have been used for years to study the molecular recognition of carbohydrates. ${ }^{152}$ Among these conjugates, polysaccharide-protein 

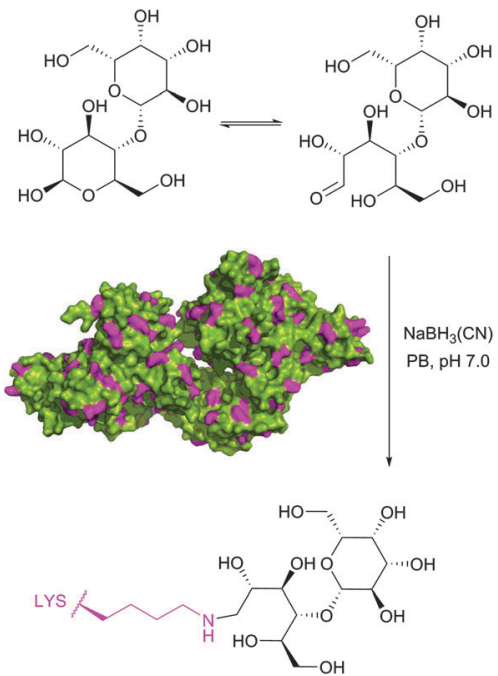

Fig. 9 First example of reductive amination of lactose by bovine serum albumin (BSA, pdb: 3V03; lysine residues are shown in magenta) described by Gray. ${ }^{147}$

conjugate vaccines such as Menactra, HIBTiter, and Prevnar are FDA approved and used routinely for the prevention of invasive bacterial infections (Fig. 10), ${ }^{20,153}$ and potential anti-infective and anti-cancer agents are currently in clinical trials. ${ }^{23,151,154,155}$ The reader is directed to a recent comprehensive review of Adamo et $a .^{24}$ covering the current status and future perspectives of carbohydrate-protein conjugates.

Another reported application of reductive amination includes the preparation of an organic trialdehyde to be used as a template for the synthesis of three-helix bundle proteins, ${ }^{156}$ protein PEGylation $^{157,158}$ and immobilisation. ${ }^{159}$

Reductive amination however possesses several drawbacks preventing it from being generally applicable to protein conjugation. ${ }^{160}$ The most important is the necessity to use watersensitive sodium cyanoborohydride, which has the potential for

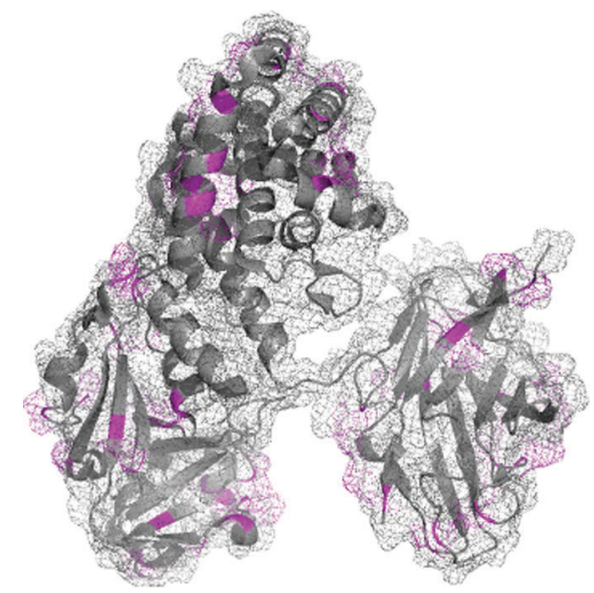

Fig. 10 Structure of Prevnar 13 vaccine. The bacterial capsule sugars, a characteristic of the pathogens, are linked to CRM197, a nontoxic recombinant variant of diphtheria toxin (pdb: 4AEO), by reductive amination at lysine residues and $\mathrm{N}$-terminus (shown in magenta).

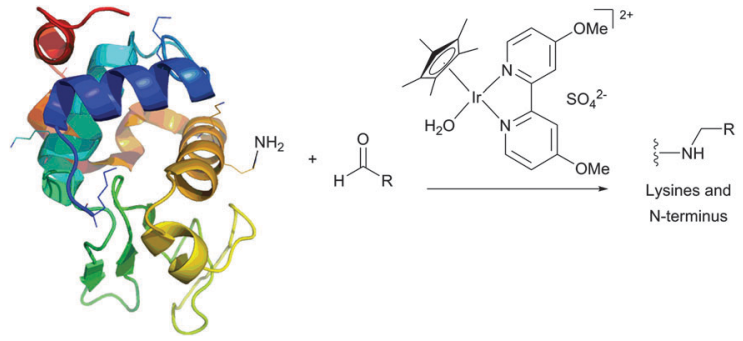

Fig. 11 Modification of lysozyme (pdb: 2LYZ) using reductive alkylation with a water-stable iridium catalyst developed by McFarland and Francis. ${ }^{161}$

reducing disulfide bonds within proteins. As an alternative, McFarland and Francis ${ }^{161}$ have reported a water-stable iridium catalyst (Fig. 11). However, the efficiency of the method is lower than that of the classical reduction with cyanoborohydride.

1.1.4 Sulfonyl halides and sulfonates. Introduced in 1952 by Weber $^{162}$ for fluorescent labelling of macromolecules, dansyl chloride (DNSC) was the first widely used sulfonyl chloride for the modification of proteins. It has gained incontestable popularity for the study of proteins after Hartley and Massey have successfully used it for the determination of the active centre of chymotrypsin. ${ }^{163}$ DNSC-Edman degradation was proposed by Gray $^{164}$ to improve the ease and reproducibility of a classical isothiocyanate-based Edman degradation. ${ }^{38}$

Sulfonyl halides are highly reactive but also very unstable, especially in aqueous media at the $\mathrm{pH}$ required for reaction with aliphatic amines. For example, Haugland and collaborators ${ }^{165}$ have demonstrated that the rate of hydrolysis of Texas Red (one of the most widely used long-wavelength fluorescent probes) ${ }^{166}$ and Lissamine rhodamine B sulfonyl chloride was much higher (complete hydrolysis within 5 minutes in $\mathrm{pH} 8.3$ aqueous solution) than that of corresponding NHS esters (both retained most of their reactivity for more than an hour under the same conditions). Yet, the formed sulfonamide bonds are extremely stable and even survive amino acid hydrolysis, ${ }^{164,167}$ which makes sulfonamide conjugates useful for the applications where the stability of the conjugation bond is a crucial feature.

Optimal conditions of protein modification by sulfonyl chlorides are those under which free amino groups most effectively compete with water for a limited amount of the reagent. It is thus best done at low temperature at $\mathrm{pH} 8.5-9.5{ }^{168}$ At lower $\mathrm{pH}$ values, the unreactive protonated form of amines slows the labelling reaction compared to the hydrolysis by water, above this range the reagent is hydrolysed too rapidly. ${ }^{169,170}$ In practical experiments, a several-fold excess of the reagent is usually added, providing the unused probe is hydrolysed to the corresponding unreactive sulfonic acid after labelling. It must be borne in mind that unlike other amine-selective reagents, sulfonyl chlorides are unstable in dimethylsulfoxide, classically used for the preparation of stock solutions, and should never be used in this solvent (Fig. 12). ${ }^{171}$

Apart from being reported for fluorescent labelling of proteins, ${ }^{172}$ sulfonyl chlorides were used to incorporate a chelate moiety into proteins, ${ }^{173}$ to study hydrodynamic properties or introduce long-lived fluorescence labels into macromolecules 


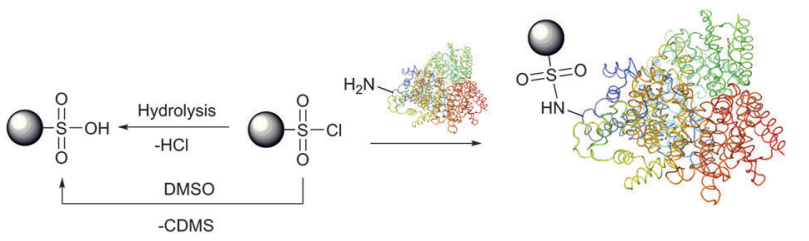

Fig. 12 Reaction of sulfonyl chlorides with amino groups present in proteins Hydrolysis of the starting material by water or dimethylsulfoxide ${ }^{171}$ (chlorodimethylsulfide, CDMS, is a leaving group) results in obtaining unreactive sulfonic acid.

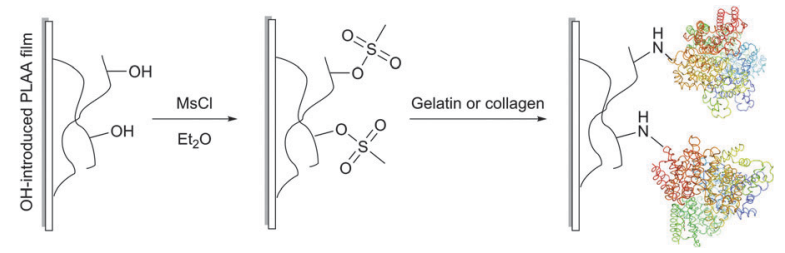

Fig. 13 Schematic representation of the reaction protocol for immobilisation of protein on PLLA film surfaces described by Ma et al. ${ }^{181}$

using tagging with pyrene derivatives ${ }^{174,175}$ or as cross-linking reagents. ${ }^{176}$

Because of their very high reactivity towards nucleophiles, sulfonyl halides also form conjugates with tyrosine, cysteine, serine, threonine, and imidazole residues of proteins; ${ }^{177}$ therefore, they are less selective than either NHS esters or isothiocyanates. These conjugates are however unstable and can be completely hydrolysed under basic conditions.

Covalent immobilisation of proteins on hydroxyl group containing carrying supports (such as agarose, cellulose, diol-silica, or polylactic acid films) is often accomplished by transforming the latter into corresponding sulfonates: tosylate, mesylate, or tresylate, ${ }^{178,179}$ serving as good leaving groups (Fig. 13). ${ }^{180-182}$ Albumin, cytokines and other therapeutic proteins and peptides were reported to undergo mild PEGylation by means of PEG tresylates. ${ }^{183-185}$ Although rather specific to amino groups, the chemistry of tresylate-mediated conjugation is not unique and well defined. For instance, Gais et al. have shown that PEGtresylate conjugation can produce a product that contains a degradable sulfamate linkage resulting in heterodispersity of the reaction. ${ }^{186}$

1.1.5 Fluorobenzenes. Despite their utmost importance for protein modification and amino group quantification since Sanger and Tuppy's work on the structure of insulin, ${ }^{187}$ derivatives of fluoronitrobenzene are of limited usefulness for bioconjugation.

Compared to other aryl halides, fluoro-substituted nitrobenzenes were found to be the most reactive in bimolecular nucleophilic substitution reactions. ${ }^{188}$ They are usually regarded as aminoselective reagents, despite their known reactivity towards thiolates, phenolates and imidazoles, as the products obtained in these reactions are either unstable at alkaline $\mathrm{pH}$ required for the reaction (Tyr and His) or can be thiolysed by excess $\beta$-mercaptoethanol (Cys). ${ }^{189}$

4-Fluoro-7-nitro-2,1,3-benzoxadiazole (NBD-F), which has been introduced as a fluorogenic reagent for more than 30 years ago by
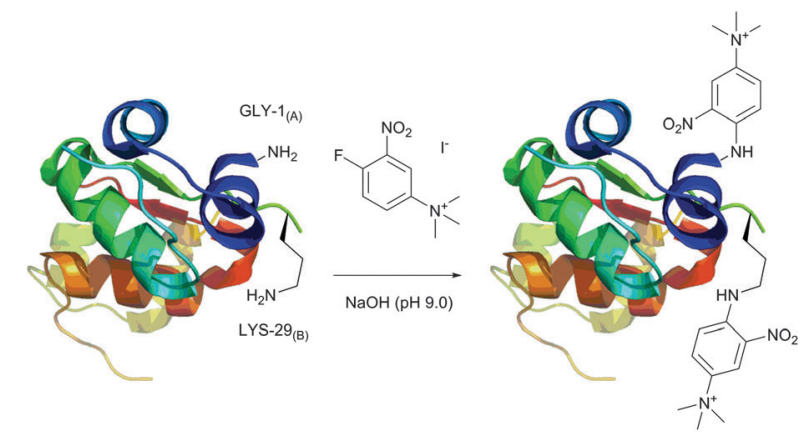

Fig. 14 Derivatisation of bovine insulin with 1-fluoro-2-nitro-4-trimethylammoniobenzene iodide described by Sutton et al. ${ }^{195}$ Only amine-containing residues of $\mathrm{A} 1$ and $\mathrm{B} 1$ chains are shown. Two of the four tyrosine residues present in chains $\mathrm{A} 1$ and $\mathrm{B} 1$ (not shown) also react with the probe under described conditions.

Imai and Watanabe, ${ }^{190}$ still remains important for several applications, mainly pre-column derivatisation and enrichment of peptides. The reader is referred to a recent review by Elbashir et $a{ }^{191}$ providing an excellent overview of the NBD-F applicability to the analysis of peptides and to a complete overview of NBD-mediated methodologies for the fluorescent labelling of amino acid residues by Imai and associates. ${ }^{192,193}$

An elegant approach for improving protein crystallizability, still remaining a major challenge in protein structure research, ${ }^{194}$ was elaborated by Sutton and collaborators ${ }^{195}$ and consists in the introduction of a charged ammonium residue. It exploits the amine-selective derivatisation of protein by 1-fluoro-2-nitro4-trimethylammoniobenzene iodide (Fig. 14) and results in increasing the hydrophilicity thereof. Using their approach, the authors were able to study the binding site ${ }^{196}$ and to obtain crystalline derivatives of modified bovine insulin, ${ }^{197}$ which is especially hard to crystallize without inducing structural changes. ${ }^{198}$ A similar protocol was used by Ladd et al. ${ }^{199}$ for chromophorical PEGylation of proteins with polyethylene glycol fluoronitrobenzene derivatives.

1.1.6 Imidoesters. First investigated by Hunter and Ludwig in $1962,{ }^{200}$ the reaction of imidoesters with peptides reached its climax for protein modification ten years after, when Traut et $a .^{201}$ introduced the 2 -iminothiolane reagent (today carrying Traut's name) for cross-linking. It allowed for producing disulfide-linked dimers of neighbouring proteins on the intact 30 s ribosome from $E$. coli using a two-step procedure: the reaction of ribosomal amino groups with the imidoester function followed by the mild oxidation of the obtained thiolate-charged ribosomes (Fig. 15). An excellent review of the cross-linking studies for the determination of ribosomal structure was published by Nomura. ${ }^{202}$

Imidoesters react with primary amines to form amidine bonds. A high specificity towards amines can be achieved when alkaline conditions ( $\mathrm{pH} 10)$ and amine-free media, such as borate buffer, are used. ${ }^{202}$ This places imidoesters among the most specific agents for amine labeling. Because the resulting amidine bonds are protonated at physiological $\mathrm{pH}$, positive charges near modified sites are preserved during the conjugation 


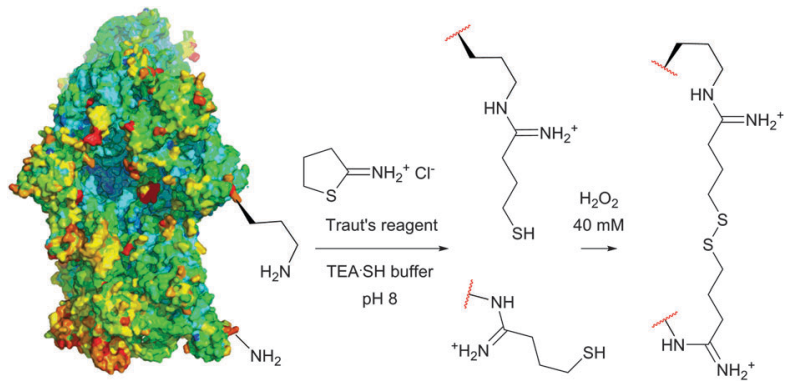

Fig. 15 Procedure of the cleavable crosslinking of the intact 30s ribosomes (pdb: 1J5E) described by Traut et al. ${ }^{201}$ Lysine residues (Lys-72 and Lys-156) were chosen randomly for simplicity purposes (TEASH stands for triethanolamine buffer adjusted to 3\% 3-mercaptoethanol).

with lysines and N-termini. Consequently, as was first demonstrated by Wofsy et al. ${ }^{203}$ such modifications produce little or no significant changes in the conformational properties and biological activities of proteins.

Thiolates obtained after the ring opening of Traut's reagent by free amines enable a plentiful thiol-selective chemistry on modified $\alpha$ - or $\varepsilon$-amino groups of proteins (see Section 1.3). Although, many imidoesters other than Traut's reagent are today commercially available (for example, see DMA, DMP, or DTBP), the amount of described labelling imidoester probes is rather scarce.

Schramm et $a .^{204}$ have described the synthesis of fluorescent imidoester dyes from corresponding nitriles; the approach was later used by Bozler et $a l^{205}$ for the preparation of dansyl containing imidoester and selective modification of lysine residues in the active site of glucose dehydrogenase. New readily available reagents for the attachment of sugars to proteins via imidoester linkage, ${ }^{206}$ hydrophilic spin probes for determining membrane protein interaction using $\mathrm{EPR},{ }^{207}$ immunoreactive probes, ${ }^{208}$ tyrosinelike probes for radioactive labelling with ${ }^{125} \mathrm{I},{ }^{209}$ protein PEGylation reagents, ${ }^{210}$ and the immobilisation of trypsin, yeast alcohol dehydrogenase, and $E$. coli asparaginase onto several types of organic polymer beads ${ }^{211}$ were achieved via imidoester conjugation and proven to have several advantages compared to other existing methodologies, namely, deprivation of solubility issues and retention of positive charge at the reaction site.

1.1.7 Miscellaneous amine-selective reagents. Several methods of amine-selective modification of proteins were not included in the main chapter, either because of scanty information available or their applicability reduced to a specific substrate type and is not general.

Azetidinone chemistry has recently been demonstrated by Barbas and collaborators ${ }^{212,213}$ to have potential for selective lysine labelling of a particular IgG framework, containing a very reactive lysine residue with an unusually low $\mathrm{p} K_{\mathrm{a}}$ of about 6 . Some detailed procedures are described for a smooth opening of a $\beta$-lactam moiety resulting in a $\beta$-alanine peptide bond. ${ }^{213}$

Discovered by Tietze et al. as two-step sequential procedures for coupling of amines, ${ }^{214,215}$ squaric acid diester amine-amine conjugation is now actively developed by Wurm et al., who have recently reported their successful use for the one-pot preparation

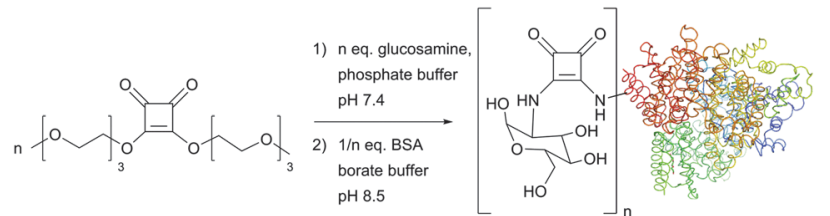

Fig. 16 One-pot, two-step squaric acid diester mediated glycosylation of BSA (pdb: 3V03) described by Wurm et al. ${ }^{217}$ Up to 22 lysine residues of the 59 present in BSA (30-35 are available for post-modification) could be glycosylated with 25 -fold excess of squaric diester and glucosamine in 12 hours.

of poly(glycerol)-protein ${ }^{216}$ and glycol-protein conjugates ${ }^{217}$ in aqueous media (Fig. 16).

Dichlorotriazine derivatives were described for amine-selective conjugation mainly as fluorescent dyes ${ }^{218,219}$ and PEGylation probes. ${ }^{220-222}$ They were shown to possess high reactivity towards protein amines. However, as was demonstrated by Abuchowski et al. ${ }^{220}$ because the hydrolysis of dichlorotriazine occurs readily under slightly basic conditions $(\mathrm{pH} 9.2)$ needed for reaction to take place with sufficient selectivity towards amines, a considerable excess of the probe must be used in the coupling reaction. Banks and Paquette ${ }^{70}$ have conducted a comparative study of three fluorescent probes, differing only in the moiety responsible for the reactivity with amines: CFSE (NHS ester), DTAF (dichlorotriazine) and FITC (isothiocyanate). It was found that the rate of conjugation is significantly faster for the NHS ester compared to the diclorotriazine probe, which, in turn, reacts faster than the isothiocyanate derivative. Each conjugate provided a satisfactory level of stability in solution over a period of 1 week at room temperature, although the hydrolysis of the remaining, relatively inert, chloro group of DTAF was observed (Fig. 17).

Arpicco et al. ${ }^{223}$ have prepared thioimidoester activated PEGcontaining derivatives and shown their superiority over the NHS-activated analogue for gelonin modification (the reaction was conducted in PBS at $\mathrm{pH}$ 7.4). In this particular case, PEGylation with a less active, compared to NHS ester, thioimidoester derivative resulted in the gelonin conjugate with higher inhibiting activity. Ikeda and associates ${ }^{224}$ have recently described a protocol for the preparation of the glutalaldehyde-functionalised PEG reagent, allowing for protein PEGylation under mild reaction conditions. Similarly, the modified protein exhibited higher biological activity than when reacted with a corresponding NHS-activated PEGylation reagent.

$\alpha$-Halocarbonyls, such as iodoacetamides, can modify lysine residues at $\mathrm{pH}>7.0,{ }^{225}$ but the reaction rate is much slower than the reaction with cysteine residues. Another class of

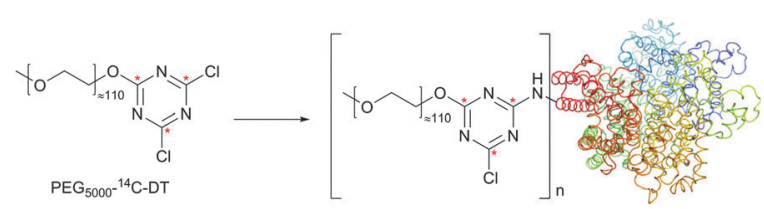

Fig. 17 Modification of BSA (pdb: 3V03) by the PEG- $\left[{ }^{14} \mathrm{C}\right]$ dichlorotriazine probe reported by Abuchowski and colleagues $\left({ }^{14} \mathrm{C}\right.$ atoms are marked with red stars). ${ }^{220}$ 
reagents usually used in cysteine-selective conjugation - vinylsulfones $^{226}$ (see Section 1.3.3) - was recently reported to be applicable for lysine labelling at slightly basic $\mathrm{pH}^{227,228}$

Modification of Lys residues with acid anhydrides, including succinic, citraconic, maleic, trimellitic, cis-aconitic, and various phthalic anhydride derivatives belongs to a pool of classically used protein modification methodologies ${ }^{229}$ and allows for transforming nucleophilic amines into acids and, as a result, enables carboxylate-selective chemistry thereof.

For more details of the practical aspects of using the abovedescribed methodologies, the reader is referred to a recent review by Brun and Gauzy-Lazo ${ }^{230}$ on the preparation of antibody-drug conjugates by lysine conjugation.

\subsection{Serine and threonine}

With $\mathrm{p} K_{\mathrm{a}}$ values $>13$, the hydroxyl groups of serine (Ser, S) and threonine (Thr, T) are rather poor nucleophiles close to physiological pH. No examples of direct conjugation of in-chain serine and threonine have been therefore reported to date.

However, highly amine selective $N$-hydroxysuccinimide (NHS) esters have been documented to give occasional side reactions with hydroxyl side chains..$^{83,90,92,231}$ In a series of experiments, Miller et al. have demonstrated that the presence of histidine in sequences of the type His-AA-Ser/Thr or His-AA$\mathrm{AA}^{\prime}$-Ser/Thr (where AA and $\mathrm{AA}^{\prime}$ stand for any amino acid) can significantly increase the reactivity of hydroxyl groups toward classical amine labelling agents (Fig. 18). ${ }^{85,86,88,232}$

Similarly, Mädler and Zenobi have reported that the guanidinium group of arginine can contribute to the reactivity of hydroxyl groups toward NHS esters and catalyse the nucleophilic substitution. ${ }^{233}$ In both cases, it is hypothesised that the imidazolyl and guanidine moieties of histidine and arginine, respectively, catalyse the reaction by stabilizing the transition state by means of hydrogen bonds and electrostatic interactions. This promoting effect is thought to be responsible for side reactions on several substrates while using cross-linking reagents. ${ }^{92,233}$

Despite the fact that methodologies of selective in-chain serine and threonine labelling are rather scarce, these residues

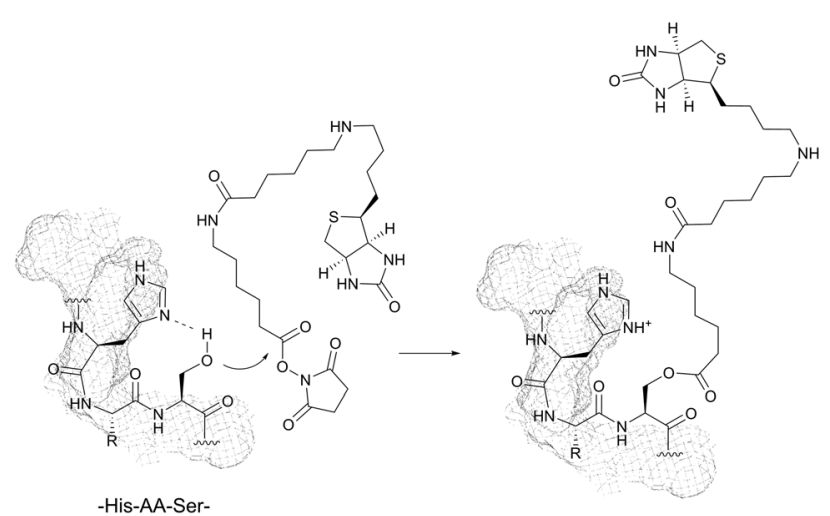

Fig. 18 Nucleophilic attack of serine on the NHS-activated ester gives the stable $\mathrm{O}$-acylated derivative. In addition to His-AA-Ser, this stable intermediate can be formed in the presence of linear sequences of His-AA-Thr and His-AA-Tyr, where AA refers to any amino acid. are of special interest for bioconjugation when located on the $\mathrm{N}$-terminus (see Section 2.2).

\subsection{Cysteine}

Cysteine (Cys, C) is perhaps the most convenient target for bioconjugation owing to the exceptionally high nucleophilicity of its sulfhydryl $(-\mathrm{SH})$ side chain which, and particularly in its deprotonated thiolate form $\left(-\mathrm{S}^{-}\right)$, largely exceeds the reactivity of any other nucleophilic function in proteins. ${ }^{234}$ Furthermore, its relative rarity in proteins present in living organisms $(1-2 \%)^{235,236}$ and the ease of its introduction into a specific site by site-directed mutagenesis allows access to protein assemblies with a single cysteine at a predetermined position. ${ }^{237}$ Even in proteins with multiple cysteines, the multiplicity is usually much smaller compared to lysines, which makes thiol-reactive labelling the preferred approach over amine-reactive methodologies.

In proteins, thiols can also be generated by selectively reducing cystine disulfides with reagents such as dithiothreitol (DTT, D1532), ${ }^{238}$ 2-mercaptoethanol ( $\beta$-mercaptoethanol), or tris[2-carboxyethyl]phosphine (TCEP). ${ }^{239,240}$ Generally, all these reagents must be removed before conducting thiol-selective conjugation, as they will compete with target thiols in proteins otherwise. $^{241}$ Unfortunately, removal of reducing agents is sometimes accompanied by air oxidation of thiols back to disulfides. Although, in contrast to the majority of thiol-reducing agents, TCEP does not contain the thiolate group, there have been several reports that it can react with $\alpha$-halocarbonyls or maleimides and that labelling is inhibited when TCEP is present in the reaction medium. ${ }^{242,243}$

Direct labelling of the thiolate group is usually achieved by either a nucleophilic addition or displacement reaction with the thiolate anion as the nucleophile. The substantially less dissociation energy of sulfhydryl groups compared to the corresponding alcohols provides much higher acidity of the former and, as a consequence, a wider availability of its slightly nucleophilic anionic form at physiological $\mathrm{pH}$.

1.3.1 $\alpha$-Halocarbonyls. First reports on the use of $\alpha$-halocarbonyl electrophiles, namely iodoacetamides, date back to 1935, when Goddard and Michaelis ${ }^{244}$ have first reported their application in modifying and studying keratin. Even today, almost 80 years later, these electrophiles are still among the most widely used for the modification of cysteine, especially in mass spectral analysis and peptide mapping of cysteine containing proteins. ${ }^{245}$ Use of iodo compounds is typical because, as iodide is a better leaving group among other halogens, these render higher reaction rates for conjugation (the relative reactivity is $\mathrm{I}>\mathrm{Br}>\mathrm{Cl}>\mathrm{F}$ ). Iodoacetyl-containing crosslinkers, biotinylation reagents, immobilisation kits, and mass spectrometry tags are now commercially available (e.g. BIAM, SIAB, UltraLink $^{\mathrm{TM}}$ Iodoacetyl Resin and Gel, iodoTMT $\left.{ }^{\mathrm{TM}}\right)$. Although corresponding maleimide reagents are more popular because of their even higher reaction rates, the haloacetyl-mediated conjugations are usually preferred for the applications where the elevated stability and compact size of the generated linkage (compared to maleimide) are crucial. Indeed, such bioconjugates 
degrade to $S$-alkyl cysteine derivatives only during amino acid hydrolysis.

Typically, the reaction of sulfhydryl groups with haloacetamides is conducted under physiological and alkaline conditions $(\mathrm{pH} 7.2-$ 9.0). When iodoacetamides are used, the reaction is preferably carried out under subdued light in order to limit free iodine generation, which has the potential to react with Tyr, His and Trp residues. The reaction is most specific for sulfhydryl groups at $\mathrm{pH}$ 8.3. The iodoacetyl group is known to react with other amino acid side chains, especially when there is no cysteine present or if a gross excess of iodoacetyl is used. For instance, free amino groups, the thioester of methionine, and both imidazolyl side chain nitrogens will react with iodoacetyl groups above $\mathrm{pH} 7$ and 5, although with much slower kinetics. ${ }^{246}$ This, however, can be resolved by the use of less reactive chloroacetamides ${ }^{247}$ or cautious control of $\mathrm{pH}$ and incubation time.

It is to be noted that the local environment has a profound effect on the reactivity of cysteine residues in proteins. If moderately reactive reagents such as iodoacetamide are used for bioconjugation, this difference in reactivities makes it possible to discern different types of Cys moieties present in the protein. Almost half a century ago, Gerwin ${ }^{248}$ reported dramatic differences in the reactivities of chloroacetic acid and chloroacetamide in the modification of the active-site cysteine of streptococcal proteinase, which was found to be due to the influence of the neighbouring histidine residue. As a general trend, cysteine residues possessing lower $\mathrm{p} K_{\mathrm{a}}$ values are more reactive when reaction is conducted under neutral or slightly acidic conditions, owing to their greater degree of dissociation and, as a consequence, higher concentration of the corresponding thiolate anions in the medium. For instance, Kim et al. ${ }^{249}$ have described a method for selective biotinylation of low-p $K_{\mathrm{a}}$ cysteine residues in proteins simply by conducting the reaction at slightly acidic pH (Fig. 19).

Davis and Flitsch ${ }^{250}$ described a procedure for the selective glycosylation of proteins at one or several sites by reacting the carbohydrate-tethered iodoacetamides with cysteine side chains, which allowed for preparing homogeneously glycosylated human erythropoietin $^{251}$ and dihydrofolate reductase. ${ }^{252}$

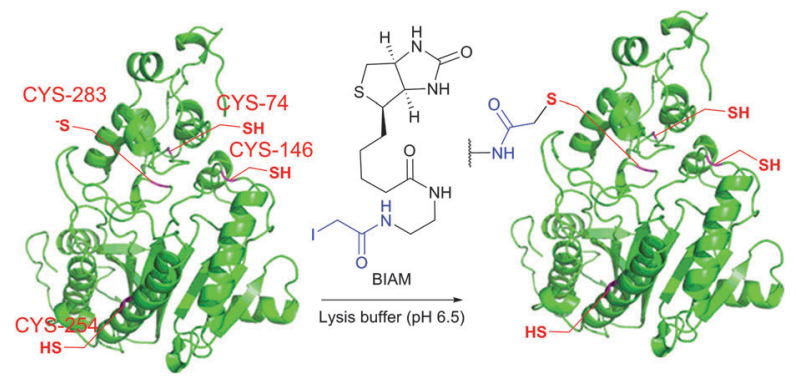

Fig. 19 Biotinylation of the low- $\mathrm{p} K_{\mathrm{a}}$ cysteine residue of rabbit muscle creatine kinase $(\mathrm{CK}, \mathrm{pdb}: 2 \mathrm{CRK})$ by $\mathrm{BIAM}^{249}$ The charge interaction between the negatively charged thiolate and the positively charged amino acid residues nearby results in a significantly lower $\mathrm{p} K_{\mathrm{a}}$ value of the CYS283 residue (6.5). Consequently, selective alkylation thereof becomes possible in the presence of three other cysteine residues with higher $\mathrm{p} K_{\mathrm{a}}$ values $(8.0-9.0)$
In 1948, Mackworth ${ }^{253}$ published his study on the reactivity of the biochemical mechanism of the lachrymatory effect of certain war gases and first reported the reactivity of structurally relevant $\alpha$-bromoacetophenones for the inhibition of several classes of thiol enzymes.

Despite advances made in the investigation of $\alpha$-haloacetophenones and related ketoximes for the modification of the active sites of enzymes, ${ }^{256-259}$ their utility for the conjugation is very limited because of various side reactions.

An interesting approach that allows photochemical conversion of cysteine into corresponding thioaldehyde and then to aldehyde thought to be formed by Norrish type II cleavage was reported by Clark and Lowe. ${ }^{254,255}$ Photolysis of the enzyme, alkylated by a bromoacetophenone derivative, results in spontaneous loss of hydrogen sulfide from the generated thioaldehyde to give the corresponding aldehyde (Fig. 20), which can either be utilised as a locus for aldehyde-selective conjugation or be transformed into the corresponding serine or glycine residue by reduction or transamination respectively.

1.3.2 Maleimides. As early as 1949, maleic acid imides (maleimides), products of the reaction of maleic anhydride and amine derivatives, were introduced by Friedmann as cysteinespecific reagents. ${ }^{260,261}$ Ever since, persistently gaining in popularity maleimide-mediated methodologies represent today perhaps the most often used functional groups for bioconjugation. This is mainly due to their exceptionally fast kinetics and significantly high selectivity toward the cysteine moiety in proteins.

The reason for such remarkable reactivity of maleimide towards thiolates is worth being discussed. In general, the electrophilicity of alkenes is defined by their ability to serve as acceptors of nucleophile's electron density, and thus interrelated to the energy of electrophile's $\pi^{*}$ orbital (its lowest unoccupied molecular orbital, LUMO). Generally speaking, the rule is simple:

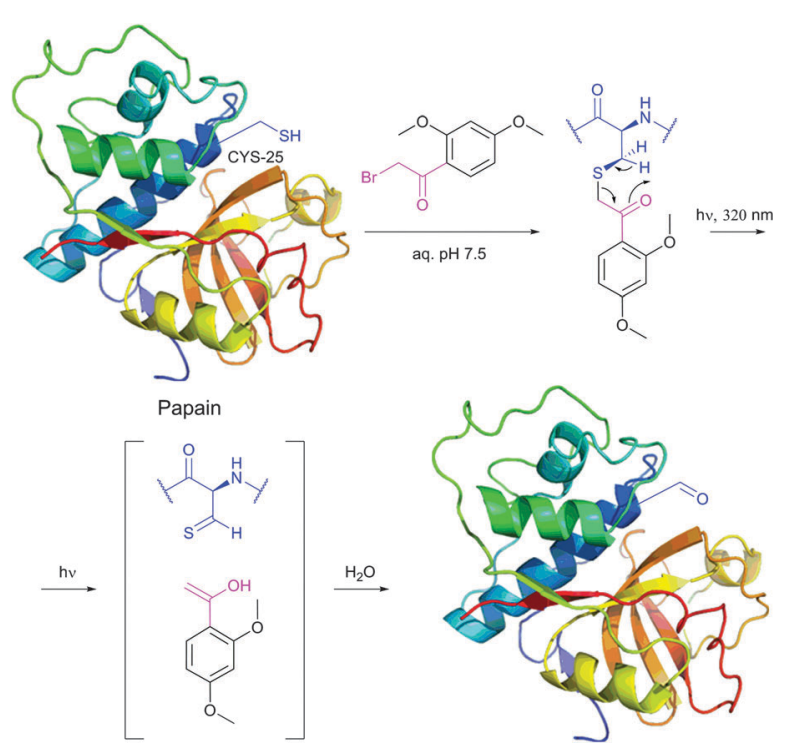

Fig. 20 Photolysis of the CYS-25 residue located in papain's active site (pdb: 1PPN) after its labelling with 2-bromo-2', $4^{\prime}$-dimethoxyacetophenone results in the formation of the unstable thioaldehyde, which readily hydrolyses to give the corresponding aldehyde. ${ }^{254,255}$ 

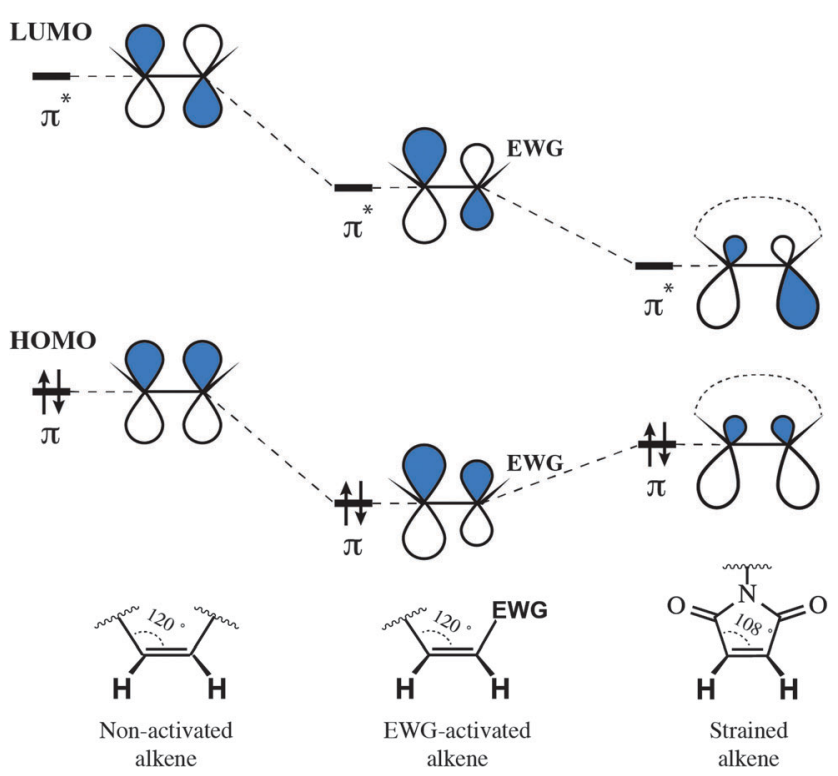

Fig. 21 Influence of an electron-withdrawing group (EWG) and cycle strain on the frontier orbitals of alkenes, $\sigma$-orbitals omitted (the form of the orbital is presented approximately, based on the publication of Merchán et $a{ }^{268}$ ). Decreasing the energy of the LUMO (lowest unoccupied molecular orbital) results in higher reactivity of the electrophile towards nucleophiles. Although via different mechanisms, both the EWG and strain of the cycle activate alkenes for the attack by nucleophiles.

the lower the energy of the alkene's $\pi^{*}$ orbital - the faster its reaction with nucleophiles. There exist two main approaches for decreasing alkene's LUMO energy: the direct attachment of an electron-withdrawing group (EWG) and the straining of the double bond. Although proceeding via two different mechanisms: by decreasing the energy of both orbitals or by diminishing the energetic gap between them, either approach results in lowering the LUMO energy of the alkene and, as a result, in the increase of its reactivity (Fig. 21). The unique reactivity of the maleimide moiety owes to the fact that it exploits these two mechanisms together. ${ }^{262,263}$

To date, a large variety of maleimide-based modifying reagents are available from a number of leading biochemical companies with even more being synthesised in laboratories around the world for specific applications. The applications of these reagents strongly overlap those of iodoacetamides, although maleimides apparently do not react with methionine, histidine or tyrosine. ${ }^{264,265}$

The optimum reaction conditions for maleimide-mediated conjugation, namely conducting the reaction under near neutral conditions (pH 6.5-7.5, Fig. 22), prevent the reaction of maleimide with amines, because the latter requires a higher $\mathrm{pH}$ to occur. At $\mathrm{pH}$ above 8 the hydrolysis of maleimide itself results in obtaining a mixture of isomeric non-strained maleamic acids unreactive toward sulfhydryls and can thus compete with thiol modification. ${ }^{28,266}$ Similarly, maleimide-thiol adducts hydrolyse, which either results in complete deconjugation or causes a significant change in the properties of the conjugate. ${ }^{266}$ Furthermore, especially at $\mathrm{pH}$ above 9 , ring-opening by nucleophilic reaction with an adjacent amine may yield crosslinked products. ${ }^{267}$

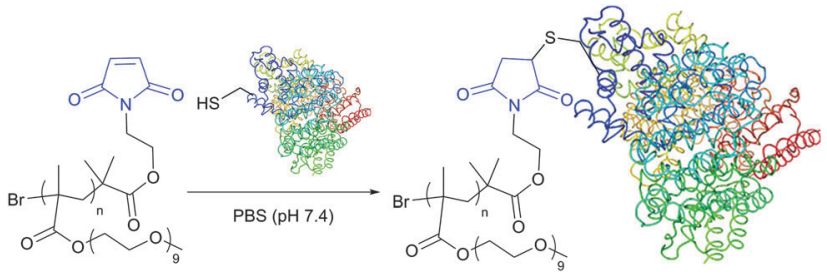

Fig. 22 Conjugation of maleimide-functional poly(PEGMA) to the only free CYS-34 residue of BSA (pdb: 3V03). ${ }^{311}$

Schuber and co-workers ${ }^{269}$ have found that important kinetic discrimination can be achieved between the maleimide and bromoacetyl functions when the reaction with thiols is conducted at pH 6.5 and 9.0, respectively.

Maleimide-NHS heterobifunctional reagents are especially important for the formation of conjugates. Hydrolysis of both the maleimide moiety and the generated thioester linkage is considerably dependent on the type of chemical group adjacent to the maleimide. Interestingly, the cyclohexane ring was found to provide increased maleimide stability to hydrolysis due to its steric effects and its lack of aromatic character. For this reason, succinimidyl-4-( $N$-maleimidomethyl)cyclohexane-1-carboxylate (SMCC) and its water-soluble analogue (sulfo-SMCC) are today among the most popular crosslinkers in bioconjugation. They are often used in the synthesis of the protein-protein or protein-probe assemblies such as antibody-enzyme or antibody-drug conjugates respectively. These include enzyme immunoassays, ${ }^{270-274}$ carrierprotein conjugates, ${ }^{275-277}$ albumin-binding prodrugs, ${ }^{278,279}$ and even approved therapies. ${ }^{117,119,280,281}$

Short homobifunctional maleimides are commonly used to explore and characterize protein structure (i.e., oligomerisation) or protein interactions. ${ }^{282-288}$ Maleimide-mediated immobilisation of biomolecules is often achieved either by direct conjugation ${ }^{13,289,290}$ or by prior biotinylation of the molecule of interest. ${ }^{291}$ The latter approach has been used for protein enrichment, ${ }^{292}$ capture, ${ }^{293,294}$ and immobilisation on modified supports. ${ }^{295-298}$

Most of the optical thiol-selective fluorescent probes often used as sensors for monitoring biological processes are represented by maleimide-containing reagents. ${ }^{299-301}$ Another testament to maleimide utility is its use in glycosylation, ${ }^{302}$ radiolabelling, ${ }^{303,304}$ studying protein interactions, ${ }^{305-308}$ and quantitation of cysteine residues. $^{309,310}$

Despite its successful application as a reagent for the chemical modification of proteins, the irreversibility of maleimide's addition makes it impossible to regenerate the unmodified protein by controlled disassembly of the conjugate. Such necessity is however often desirable for in vitro or in vivo applications. Several studies were devoted to a mild and specific hydrolysis of the imido group in maleimide conjugates. ${ }^{312,313}$ These approaches turned the originally irreversible maleimide-mediated thiol conjugation into the cleavable methodology. However, the harsh reaction conditions of this cleavage (strong basic conditions or the presence of a high amount of imidazole) make them incompatible with many fragile protein substrates.

Monobromomaleimide derivatives, introduced by Baker et al. ${ }^{314}$ in 2009, have expanded the class of reagents for the selective and 


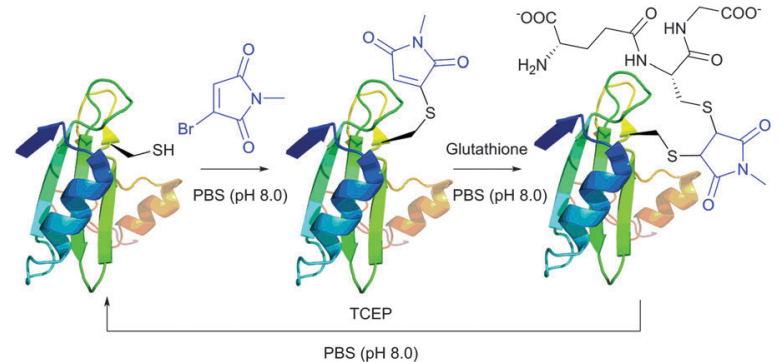

Fig. 23 Conjugate addition of the L111C mutant of the SH2 domain of Grb2 (wild-type pdb: 1JYU) to bromomaleimide followed by second addition of glutathione resulting in the generation of the vicinal biscysteine adduct. ${ }^{316}$

reversible modification of cysteine (Fig. 23). In contrast to methanethiosulfonates (see Section 1.3.6), ${ }^{315}$ monobromomaleimides allow much more stable conjugation of thiolates, which are easily cleavable upon reaction with TCEP by the addition-elimination sequence (Fig. 23).

Moreover, the initial modification of a protein resulted in obtaining a thiol-maleimide moiety, which was shown to be prone to a second thiol addition and resolved another recognised drawback of maleimide-based methodologies, namely the presence of only two points of attachment. ${ }^{316}$ Similar to a non-substituted maleimide, the hydrolysis of thiol-maleimide linkage results in a dramatic decrease in its reactivity towards thiolates, which can be used for "switching off" the linker after the first thiol addition (Fig. 24). ${ }^{317,318}$

1.3.3 Vinyl sulfones. The Michael-type addition of vinyl sulfones (VS) is an attractive strategy for protein conjugation, because of the elevated water stability of VS function and almost quantitative yields of their reaction with thiolates. ${ }^{319-323}$ The reaction of vinyl sulfones with lysine residues has been reported, ${ }^{320,322}$ however, occurring only at high $\mathrm{pH}$ values $(\mathrm{pH}>9.3)$.

Initially, VS-mediated approaches have been used almost exclusively for PEGylation of proteins with end-functionalised PEG derivatives. ${ }^{322,324}$ Several studies on the immobilisation of macromolecules on solid supports using vinyl sulfones were reported, owing to the elaboration of new methods for the preparation of VS-modified surfaces. ${ }^{319,320,325-327}$ Versatile VScontaining probes, namely carbohydrates, ${ }^{227,328}$ chelating agents, ${ }^{329}$ fluorescent tags, ${ }^{330,331}$ and biotinylation reagents ${ }^{331}$ were recently developed and applied in the bioconjugation of proteins (Fig. 25). Ovaa and co-workers ${ }^{332}$ used vinyl sulfone

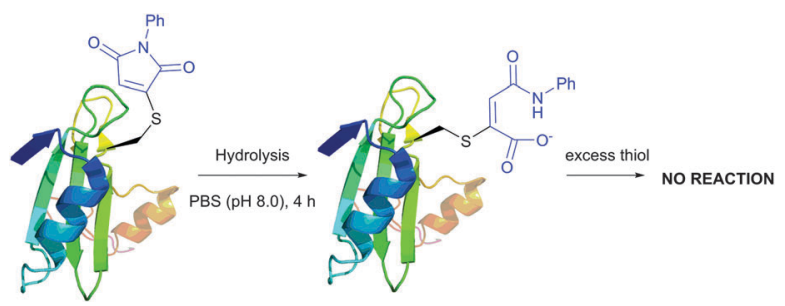

Fig. 24 "Switching off" the linker's reactivity for second thiol addition achieved by hydrolysis of the thiomaleimide linkage on the L111C mutant of the SH2 domain of Grb2 (wild-type pdb: 1JYU). ${ }^{317}$

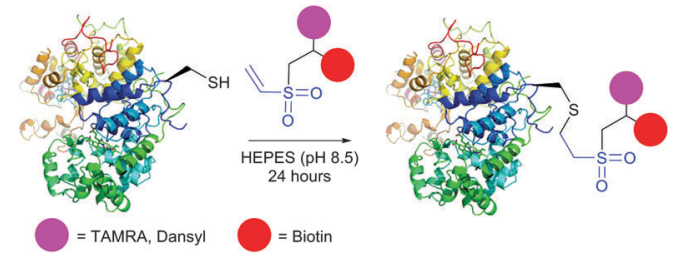

Fig. 25 Bifunctional labelling of horseradish peroxidase (HRP, pdb: 2ATJ) with vinyl sulfone-functionalised tags described by Morales-Sanfrutos et al. ${ }^{330}$

handle to conjugate enzymes to a ubiquitin-like protein. The applications of VS-tags in proteomics have recently gained popularity and have been reviewed by Lopez-Jaramillo et al. ${ }^{331}$

Vinyl sulfones react with thiols to form a stable thioether linkage to the protein under slightly basic conditions ( $\mathrm{pH} 7-8$ ). ${ }^{228}$ The reaction may proceed faster if the $\mathrm{pH}$ is increased, but this usually also increases the amount of side-products (namely the modification of the Lys $\varepsilon$-amino groups and the His imidazole rings). ${ }^{320}$ The main advantage of VS-tags is their elevated stability in aqueous solutions, compared to more reactive thiols and maleimides, which can be subjected to ring opening or addition of water across the double bond. ${ }^{185}$

1.3.4 Thiol-ene coupling. Discovered at the beginning of the last century by Posner, ${ }^{333}$ free-radical-based hydrothiolation of terminal alkenes, also called the thiol-ene coupling reaction (TEC), has emerged as a powerful approach for the chemoselective modification of both peptides and proteins. ${ }^{334,335}$ The initial step of the reaction is light- and/or initiator-induced generation of the thiyl radical. This adds to alkene in an anti-Markovnikov fashion to yield the thioalkyl radical. This leads to the propagation of the radical chain by abstraction of hydrogen from the other thiolate (Fig. 26).

The TEC conjugations (usually conducted in PBS-DMSO buffer at $\mathrm{pH}$ 7.0-7.5) are compatible with oxygen and aqueous media and are usually carried out upon irradiation at $\lambda_{\max }$ $365 \mathrm{~nm}$ in the presence of Vazo44 (2,2'-azobis[2-(2-imidazolin2-yl)propane]dihydrochloride) as an initiator. The resulting thioether linkage is biologically stable and robust.

The first approach to protein conjugation namely glycosylation via TEC was reported by Davis et al. in 2009. ${ }^{336}$ However, it consisted of photoinduced coupling of various glycosyl thiols with site-specifically introduced unnatural L-homoallylglycine. A complementary approach to peptide and protein glycoconjugation by photoinduced coupling on cysteines was first introduced almost at the same time by Dondoni and co-workers. ${ }^{337}$

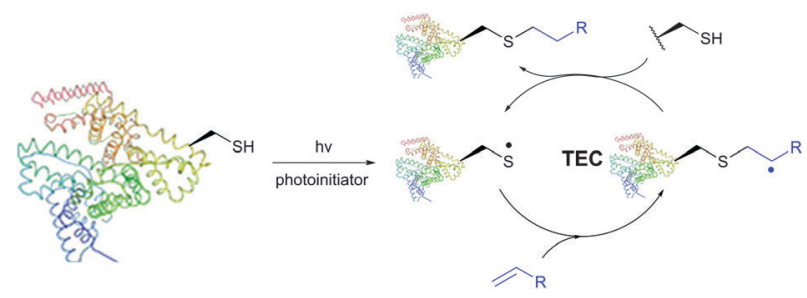

Fig. 26 Mechanism of photoinduced free-radical thiol-ene coupling (TEC). 
The $66 \mathrm{kDa}$ globular bovine serum albumin (BSA) possessing one free CYS-34 residue was selected as a model protein. Surprisingly, it revealed that not only the one CYS-34 SH group, as expected, but also two more $\mathrm{SH}$ groups arising from the $75 \leftrightarrow 91$ disulfide bond were modified. It was suggested that such hyperglycosylation was due to well-documented disulfide bond degradation by UV-irradiation, ${ }^{338}$ namely to an electron transfer process from photoexcited tryptophan residues. Furthermore, prolonged irradiation of the reaction mixture up to 2 hours induced the introduction of seven glycoside residues into BSA. Despite the necessity for UV-irradiation, ensuing side-reactions, and often moderate yields, ${ }^{335}$ the fact that, in contrast to the majority of thiol-selective methodologies, TEC does not exploit elevated nucleophilicity of the thiolate but its readiness for the generation of radicals makes it especially tolerant to a wide range of functional groups. For instance, Garber and Carlson ${ }^{339}$ have used this feature of TEC for selective capping of thiols in the presence of thiophosphorylated groups, free alcohols and amines.

Several approaches involving the combination of cysteineselective methodologies have been recently reported. Stolz and Northrop $^{340}$ studied the reactivity of $N$-allyl maleimides and found this scaffold to be appropriate for consecutive two-step conjugation of thiols: via (1) base-initiated Michael-addition to maleimide moiety and (2) radical-mediated TEC of allyl-fragment. Scanlan and associates ${ }^{341}$ developed the sequential NCL-TEC approach (for more details on NCL, see Section 2.3.1) for the functionalisation of the cysteine thiolate generated at the ligation site during native chemical ligation.

1.3.5 Thiol-yne coupling. After the rise of TEC for bioconjugation, its sister reaction of the hydrothiolation of alkynes, also referred to as thiol-yne coupling (TYC), began to receive increased attention. ${ }^{342}$ Discovered in 1949 by Jones and collaborators, ${ }^{343}$ TYC allows introduction of two thiol fragments across a carbon-carbon triple bond via a free-radical mechanism similar to TEC. The first step of the anti-Markovnikov addition of a thiyl radical to the triple bond yields an intermediate vinyl thioether capable of undergoing a second addition of the thiyl radical through the same mechanism, leading to the 1,2-dithioether (Fig. 27).

TYC occurs under the same reaction conditions as TEC and smoothly proceeds at room temperature in aqueous solutions. First trials on the applicability of TYC on peptides were reported by Dondoni and collaborators in $2010 .^{344}$ The authors have demonstrated the possibility of dual glycosylation of a series

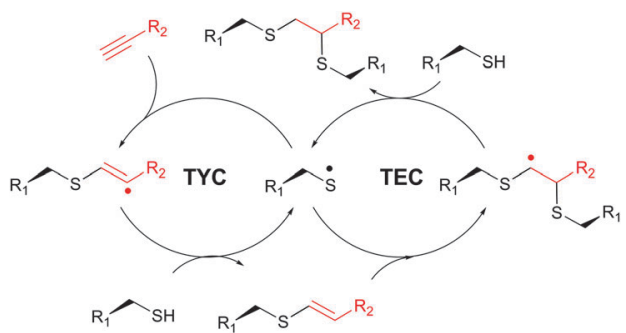

Fig. 27 Mechanism of photoinduced free-radical thiol-yne coupling (TYC).

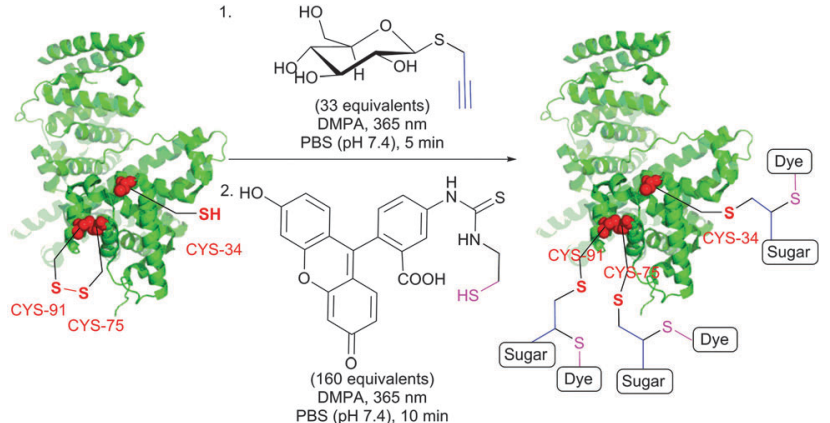

Fig. 28 Glycosylation and fluorescent labelling of bovine serum albumin (BSA, pdb: 3V03) by TYC (first step) followed by TEC (second step) with 2,2-dimethoxy-2-phenylacetophenone (DMPA) as a photoinitiator. ${ }^{345}$

of peptides (up to 8 residues). Later on, Davis and Dondoni have expanded the dual conjugation strategy for achieving sequential glycosylation and fluorescent labelling of BSA (Fig. 28). ${ }^{345}$ Just as with TEC, the reaction also occurs at cysteine residues of the $75 \leftrightarrow 91$ disulfide bridge.

The necessity of using a photo- or a chemical initiator in both TEC and TYC conjugations represents the main drawback of these methodologies, as the presence of free radicals results in a series of side reactions, namely oxidation and crosslinking of proteins.

1.3.6 Disulfide reaction. Simple air oxidation of two thiolates to form a disulfide bond is probably the most straightforward among cysteine-selective conjugation techniques. Very simplistically, it consists of open to air stirring of a protein possessing a free cysteine residue with a thiol-containing probe for several days under basic conditions. $^{346}$ Apparently, a large excess of the thiolate-probe is required in order to reduce the likelihood of protein dimerisation. Treatment with iodine ${ }^{347}$ was reported for the activation of cysteine formation of mixed disulfides. However, restricted control of product distribution and long reaction times largely limits the applicability of these methods for bioconjugation.

Diverse disulfides have been extensively used in the past decade for the modification of cysteine by disulfide exchange. This reversible reaction involves attack of cysteine thiolate at the disulfide, breaking the $\mathrm{S}-\mathrm{S}$ bond, and subsequent formation of a new mixed disulfide. A well-known example of such reaction is colorimetric quantitation of free sulfhydryls with Ellman's Reagent. ${ }^{348}$ Several symmetric disulfide-containing fluorescent probes such as BODIPY L-cystine and fluorescein L-cystine are commercially available. However, because there is no thermodynamic preference for this disulfide exchange to pass one way or another, labelling with non-activated disulfides generally requires use of a large excess of the probe to achieve sufficient levels of tagging. ${ }^{349}$ In contrast, related activated thiols, namely thiosulfates (R-S-SO ${ }_{3}{ }^{-}$), thiosulfonates (R-S-SO ${ }_{2}-\mathrm{R}^{\prime}$, MTS), sulfenyl halides (R-S-X), ${ }^{350}$ pyridyl disulfides, ${ }^{351,352}$ and TNB-thiols (derivatives of 5-thio-2-nitrobenzoic acid) contain good leaving groups, which tautomerise to give unreactive forms thus shifting the reaction equilibrium (Fig. 29).

PEGylation, fluorescent and biotinylation probes containing thiosulfate (commercialised as TS-link reagents) and pyridyl 
a)

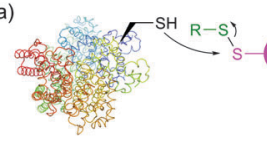

b)

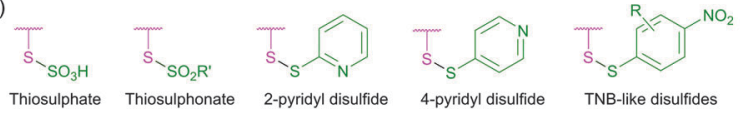

Fig. 29 (a) General scheme of protein labelling via the formation of a disulfide bond. (b) Most relevant examples of activated thiols: thiosulfate, thiosulfonate, 2-pyridyl disulfide, 4-pyridyl disulfide, TNB-like thiol.

disulfide motifs are today widely commercially available. Thiosulfonates were first introduced for bioconjugation by Davis and co-workers ${ }^{353,354}$ in their work on the controlled glycosylation $^{355}$ and further elaborated by Zhao et al. ${ }^{356}$ for site-selective PEGylation of proteins. Recent advances resulted in the further development of thiol activated methodologies towards selenenylsulfides $^{357,358}$ and even methanedithiosulfonates, allowing for synthesizing trisulfide conjugates. ${ }^{359}$ Disulfide-based conjugation was recently reported for the preparation of antibodydrug conjugates and studying the influence of the spacer length on their stability. ${ }^{360}$ Diselene analogues of disulfide PEGylation reagents were proposed by Jevševar et $a l^{361}$ as selective and fast alternatives for coupling. Although high conversion yield required the use of a large molar excess of the probe, this elegant approach represents an interesting technology which deserves further investigation.

The main factor that has gained popularity to methodologies yielding disulfide and selenol-sulfide linkage is the reversibility they afford. However, the resulting conjugates are generally less stable than those obtained using bromomaleimides ${ }^{315}$ (Section 1.3.2) and can be readily cleaved with classical reducing agents such as DTT, $\beta$-mercaptoethanol or TCEP. Yet, in the case of disulfides, the modified protein can be made more stable and resistant to reduction by the corresponding thioether-linked conjugate by means of HMPT-mediated desulfurisation elaborated by Davis and associates (Fig. 30). ${ }^{362}$ The use of hindered disulfides represents another way to increase the resistance of generated conjugates to cleavage. ${ }^{363}$

1.3.7 Disulfide rebridging. Use of thiol reactive reagents often requires the necessity for recombinant introduction of a free cysteine into the protein ${ }^{364}$ because most proteins do not have a free cysteine. ${ }^{365,366}$ This new free unpaired cysteine may cause disulfide scrambling, complicate protein refolding, ${ }^{364}$ or lead to aggregation of the protein. ${ }^{367}$ In contrast, most of the

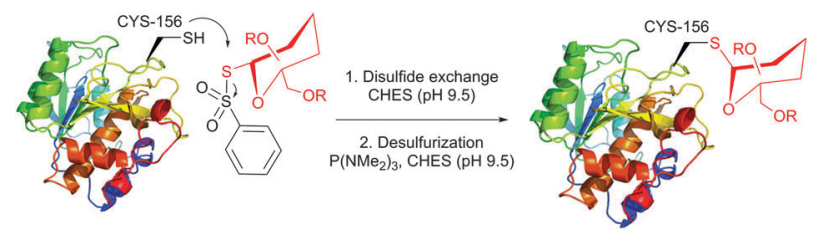

Fig. 30 Two-step protocol for the preparation of thioether linkage via disulfide exchange reaction followed by HMPT-mediated desulfurisation $\left(\mathrm{P}\left(\mathrm{NMe}_{2}\right)_{3}\right.$, hexamethylphospotriamide) of the glycosylated mutant S156C SBL (wild-type pdb: 1GCI). ${ }^{362}$

biologically relevant proteins possess at least one disulfide bond in their structure. ${ }^{368}$ The direct reduction of disulfide bonds followed by conjugation with thiol-selective reagents is, however, usually inadmissible, since these are responsible for their structure, stability, or function ${ }^{369,370}$ and must thus remain bridged after the modification in order not to alter protein tertiary structure.

In an attempt to resolve this problem, Brocchini et al. ${ }^{371}$ developed a clever methodology for the PEGylation of protein disulfide bonds with $\alpha, \beta$-unsaturated bis-thiol alkylating reagents. Covalent rebridging of the two thiols derived from the disulfide after its mild reduction allowed obtaining the modified proteins with retained tertiary structure and biological activity. Interferon $\alpha-2 b$ (IFN) was used in the initial studies, because it is representative of four-helical-bundle proteins with accessible disulfide bonds. Following reduction of the disulfide in IFN's, the two free cysteines were re-joined using a threecarbon linked functional PEG. ${ }^{368,371}$ The methodology was further expanded to PEGylation of therapeutic proteins, ${ }^{368,372,373}$ antigen-binding fragments of immunoglobulin $\mathrm{G},{ }^{374}$ and poly phosphocholine labelling of IFN. ${ }^{375}$ Simultaneously with the introduction of previously mentioned monobromomaleimides, Baker and co-workers have introduced a relevant class of reagents containing a highly reactive dibromomaleimide or dibromopyridazonedione scaffold, allowing rapid and efficient disulfide rebridging by installing a rigid two-carbon linker. ${ }^{316,376}$ This approach was first applied for equimolar PEGylation of 32-amino acid salmon calcitonin (sCT, Fig. 31) (377 $^{37}$ and very recently for the preparation of homogeneous antibody conjugates. ${ }^{378,379}$

Although being very rapid (full conversion is achieved in less than 5 minutes), dibromomaleimide-based conjugation resulted in obtaining a small amount of the multimers while modifying complex polypeptides. ${ }^{378}$ Developed by Baker et al. ${ }^{380}$ and Haddleton et al. $^{381}$ more stable and less reactive dithiophenolmaleimides allowed avoiding this apparent drawback of dibromomaleimide probes. In combination with benzeneselenols known for their efficiency in the catalysis of disulfide cleavage, the dithiophenolmaleimide approach allowed selective antibody fragment conjugation with no detectable formation of multimers and conserving a high reaction rate (Fig. 32). ${ }^{378}$ Haddleton and colleagues $^{382}$ have recently reported an in situ one-pot preparation of oxytocin-polymer conjugates using dithiophenolmaleimidecontaining probes.

Structurally similar to dibromomaleimides, but containing four attachment points instead of three, dibromopyridazinediones (PD)

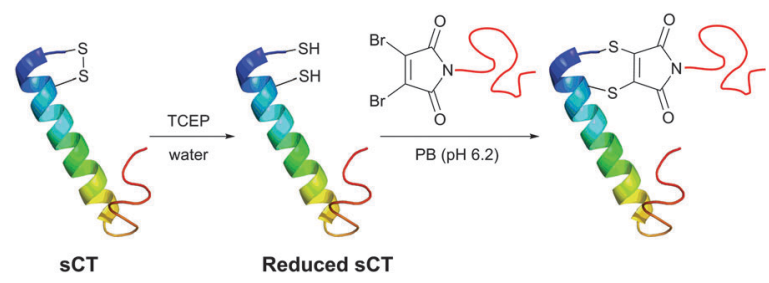

Fig. 31 One-pot reduction-PEGylation of the disulfide bridge of sCT (pdb: 2GLH) followed by rebridging using dibromomaleimide. ${ }^{377}$ 

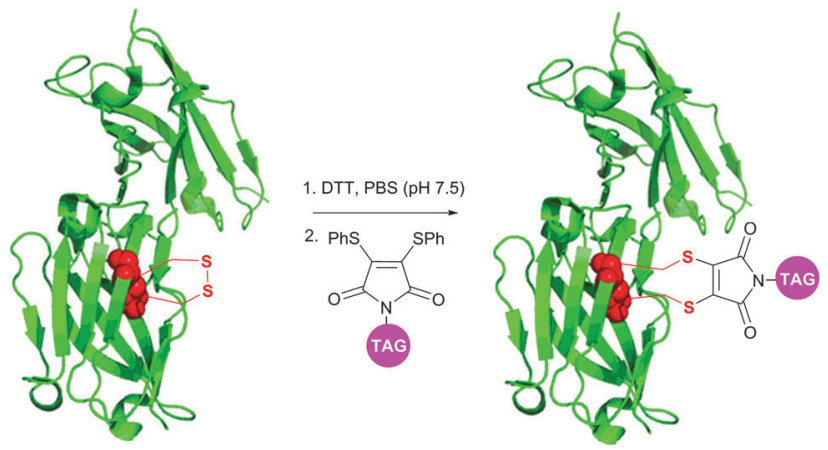

Fig. 32 Dithiophenolmaleimide approach for in situ disulfide bridging of antibody fragments (pdb: 1QOK). ${ }^{378}$

were recently described by Caddick et al. ${ }^{383,384}$ and provided a platform for IgG antibody dual labelling. ${ }^{384}$ Namely, the authors elaborated the preparation of a PD-linker containing two orthogonal reactive handles in its structure: (1) a strained alkyne, which readily reacts with azides in Cu-free strain-promoted azide-alkyne cycloaddition (SPAAC), and (2) a terminal alkyne, which reacts with azides in Cu-catalysed azide-alkyne cycloaddition (CuAAC). The construct obtained after rebridging a reduced antibody with the PD-linker was then used to selectively introduce two distinct functionalities (Fig. 33).

An interesting disulfide stapling-unstapling strategy using dichloro-s-tetrazine was developed by Smith and collaborators (Fig. 34). ${ }^{385,386}$ In addition to their ability to be photochemically cleaved (i.e., unstaple thus regenerating reduced disulphide bonds; Fig. 34a), $S, S$-tetrazine macrocycles provide a possibility for labeling by exploiting the reactivity of the tetrazine in the inverse electron demand Diels-Alder reaction (Fig. 34b).

Organic arsenicals (similar to tetracysteine-selective biarsenical dyes initially developed by Tsien and co-workers, see Section 4) were recently exploited for efficient protein-polymer conjugation (Fig. 35) ${ }^{387}$ It is noteworthy that, in contrast to highly thiol-reactive
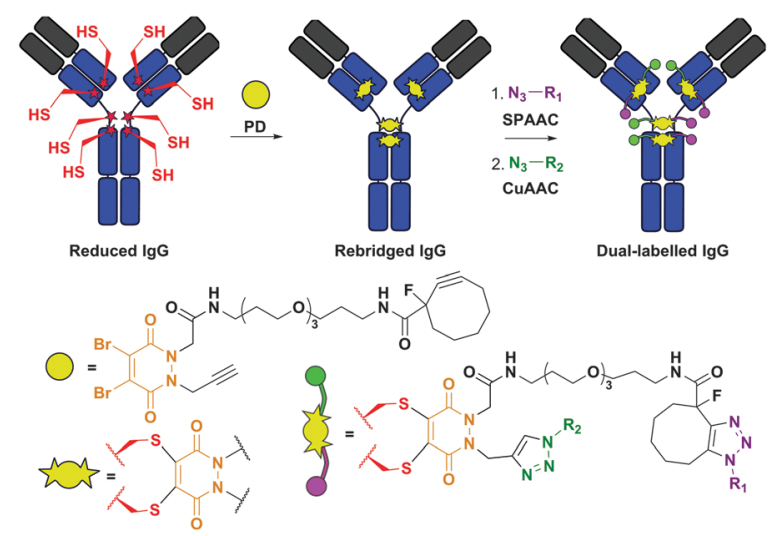

Fig. 33 Dual labelling of IgG antibody using dibromopyridazinediones (PD, shown in orange). ${ }^{384}$ The construct obtained after rebridging of a reduced IgG with a PD-probe containing a strained alkyne (reactive in strain-promoted azide-alkyne cycloaddition, SPAAC) and a terminal alkyne (reactive in Cu-catalysed azide-alkyne cycloaddition, CUAAC) was subsequently labelled with two azide-containing probes $\left(N_{3}-R_{1}\right.$ and $\mathrm{N}_{3}-\mathrm{R}_{2}$ ).

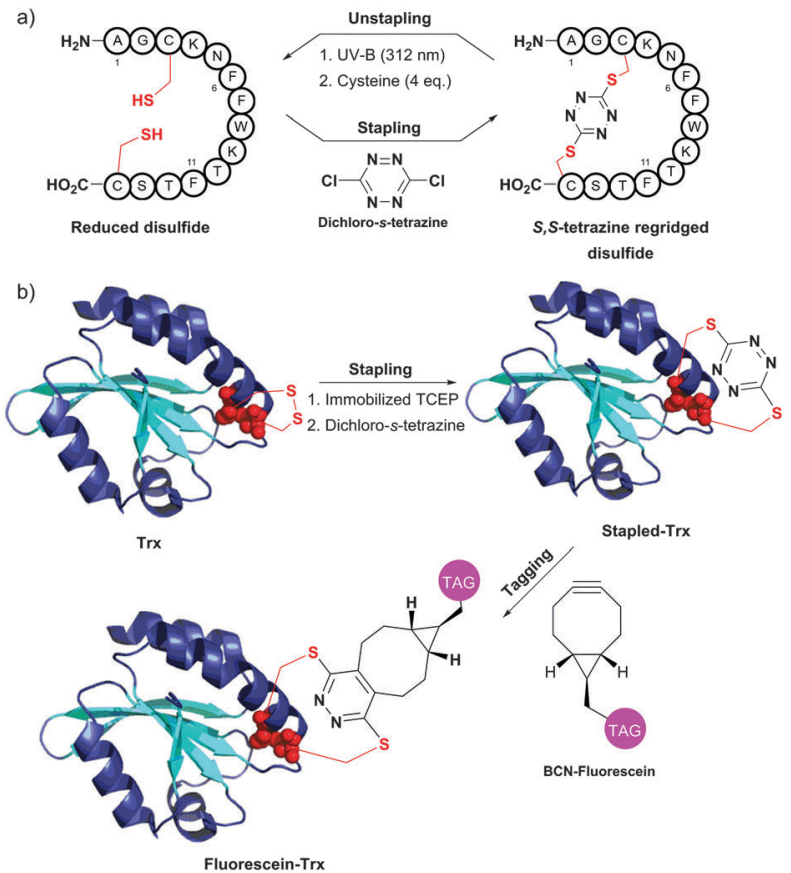

Fig. 34 Protein stapling-unstapling using dichloro-s-tetrazine described by Smith et al. (a) Rebridging of a disulfide bond of a 14-mer peptide using dichloro-s-tetrazine and recovery of the starting product by photocleavage of the resulting S,S-tetrazine macrocycle (irradiation at $312 \mathrm{~nm}$ ) followed by quenching of the thiocyanate intermediate with an excess of cysteine. (b) Rebridging of thioredoxin (Trx, pdb: 2F51) after the disulfide bond reduction followed by tagging of the resulting S,S-tetrazine with a strained alkyne containing probe (BCN-fluorescein).

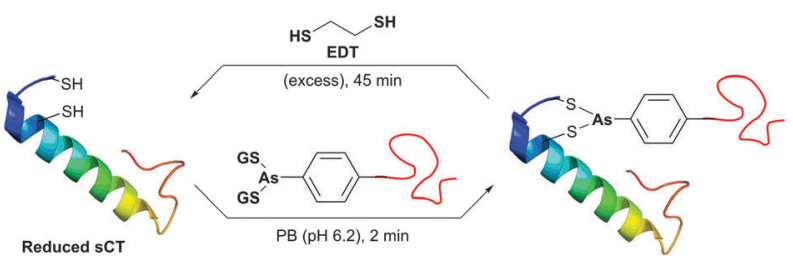

Fig. 35 Rebridging of reduced $\mathrm{SCT}$ (pdb: $2 \mathrm{GLH}$ ) with an organic arsenical $\left((\mathrm{GSH})_{2}-\mathrm{As}_{(\mathrm{II}) \mathrm{PPEGA}} \mathrm{P}_{40}\right)$ followed by release of $\mathrm{SCT}$ upon addition of ethanedithiol (EDT) described by Wilson et al. ${ }^{387}$

dibromomaleimides, these reagents demonstrated enhanced selectivity for disulfide rebridging in the presence of free Cys residues. Namely, while dibromomaleimides reacted near quantitatively within 30 minutes with the free CYS-34 of native BSA, organic arsenicals exhibited limited reactivity and demonstrated only about $20 \%$ labeling over the same period of time. ${ }^{387}$ The authors hypothesised entropy-driven affinity of arsenicals for closely spaced dithiols to be the main reason of such specificity.

1.3.8 Transforming to dehydroalanine. $\beta$-Elimination of thiolate from the cysteine moiety turns this one of the strongest nucleophilic side chain into a dehydroalanine moiety (Dha, Fig. 36) representing an electrophilic centre for reactions with nucleophiles. Such "umpolung" in terms of nucleophilicityelectrophilicity opens an extremely interesting prospect for the 
a)

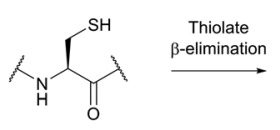

b)

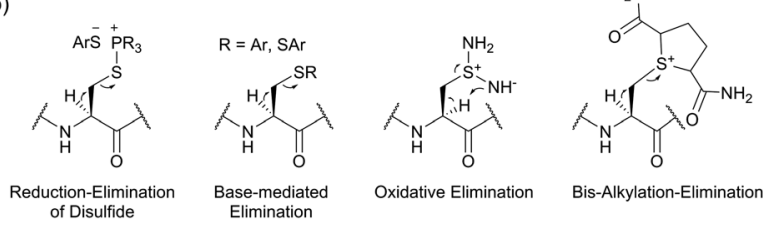

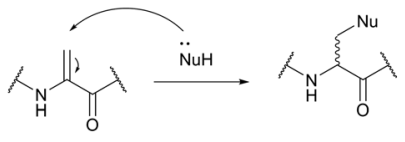

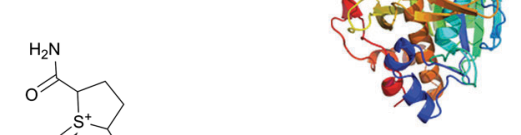

S156C-SBL

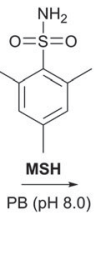

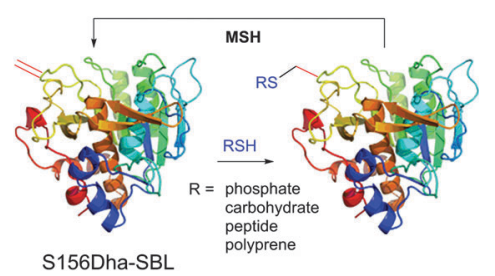

Fig. 37 Conjugation of mutant S156C SBL (wild-type pdb: 1GCI) containing a single, surface-exposed cysteine residue CYS-156 by oxidative elimination followed by conjugation with thiol probes. ${ }^{392}$
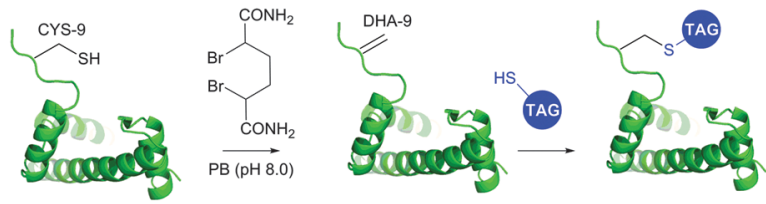

Fig. 38 Dehydroalanine-mediated conjugation on histone 3 mutant H3. ${ }^{393}$

only compatible with a restricted number of protein substrates. Only recently have Davis and collaborators ${ }^{395}$ reported a more general method for Cys $\rightarrow$ Dha transformation by means of water-soluble $\alpha, \alpha^{\prime}$-dibromoadipyl(bis)amide (DBAA) allowing generation of Dha moiety under mild conditions $\left(37^{\circ} \mathrm{C}, \mathrm{pH} 7.0-8.0\right)$ at sufficiently high yields. This approach was evaluated on several model proteins, including SBL (see above), ${ }^{395}$ the single-domain antibody cAb-Lys3 A104C mutant, ${ }^{395}$ histone H3 (Fig. 38), ${ }^{393}$ AurA kinase domain, ${ }^{402}$ and GFP mutant. ${ }^{403}$

1.3.8.3 Other approaches. Despite recent advances in dehydroalanine-mediated conjugation methodologies, the inherent limitations of these methods preclude their general use for peptide and protein modification. None of these approaches enable general, chemo- and site-selective incorporation of dehydroalanine into proteins without the need for prior incorporation of an accessible Cys residue. Several other approaches continue to appear and are designed to overpass this problematic. These are oxidative elimination of aryl-selenocysteine, ${ }^{399,404-408}$ utilising of lacticin synthetase ${ }^{409}$ transformation to selenocysteine thioethers. ${ }^{410}$

1.3.9 Miscellaneous thiol-selective reagents. An example of a simple alkylation reaction that still remains relevant in bioconjugation is aminoethylation. Known for more than half a century, ${ }^{411,412}$ it allows transforming cysteine thiolates into lysine mimicking thialysine residues by means of bromoethylamines or aziridines. Obtained thialysines were validated as appropriate synthetic substrates for further amine-selective transformations (see Section 1.1). Furthermore, the method was recently demonstrated appropriate for providing the access to more peculiar methylated lysine analogues. ${ }^{413}$ The reaction is typically conducted at $\mathrm{pH}>8.5$ to ensure a high level of cysteine deprotonation.

$\mathrm{S}_{\mathrm{N}} \mathrm{Ar}$ substitution chemistry approaches for cysteine modification in proteins were reported by several research groups. ${ }^{395,414,415}$ Davis et al. ${ }^{395}$ utilised Mukaiyama's reagent (2-chloro-1-methylpyridinium iodide) to generate an arylated cysteine as an intermediate for 

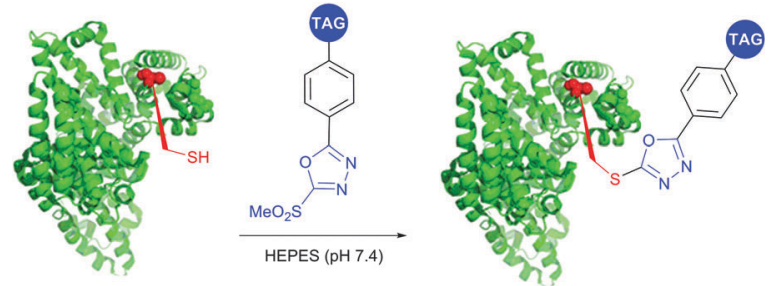

Fig. 39 Labelling of the free cysteine residue of BSA (pdb: 3V03) with Julia-Kocienski-like reagents. ${ }^{417}$

conversion thereof to dehydroalanine. Pentelute and co-workers have expanded the approach towards perfluoroaryls for protein stapling and conjugation. ${ }^{414,415}$ Finally, Barbas and associates ${ }^{416}$ have developed a class of Julia-Kocienski-like methylsulfonylfunctionalised reagents, that reacts rapidly and specifically with thiols under biologically relevant $\mathrm{pH}$ (5.8-8.0). Notably, the resulting conjugates possess superior hydrolytic stability compared to cysteine-maleimide, which makes this methodology appropriate for the preparation of stable protein conjugates and PEGylated proteins (Fig. 39).

A strategy exploiting selective cyclization of peptides containing three cysteines to generate combinatorial libraries of cysteine-rich bicyclic peptides was recently developed. This approach is based on utilisation of homotrifunctional linkers: TBMB (tribromomethyl-), TATA (triacryloyl-), or TBAB (tribromoacetamide-containing reagents). ${ }^{418,419}$

An efficient gold-catalysed allene-mediated coupling reaction has been recently developed by Che and colleagues. ${ }^{420}$ The method allowed direct thiol-selective functionalisation of model peptides and reduced RNase A (Fig. 40).

Reactions of thiols with electron deficient acetylenes have been known for decades, being, however, mostly conducted in organic solvents. ${ }^{421-425}$ Several examples of reactions in aqueous media have been recently reported. ${ }^{417,420,426,427}$ Che and co-workers ${ }^{417}$ have elaborated a versatile method for the selective cysteine labelling of unprotected peptides and proteins in aqueous media with arylalkynone reagents. Notably, modified peptides could be converted back into the unmodified peptides by treatment with thiols under mild reaction conditions (Fig. 41).

Interestingly, in contrast to arylalkynones, structurally similar electron deficient acetylenes - 3-arylpropiolonitriles (APN) - were recently reported as a prominent class of reagents for irreversible tagging of cysteine. ${ }^{428,429}$ A superior stability of resulting conjugates in aqueous and biological media opens an interesting prospect in many fields where stability of obtained conjugates is crucial, e.g. for preparation of antibody conjugates possessing increased plasma stability (Fig. 42 ). ${ }^{429}$

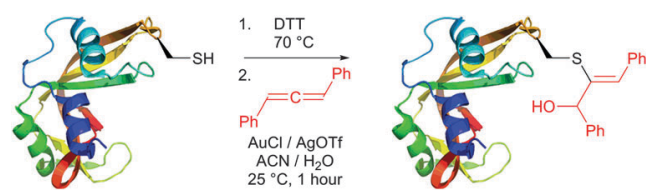

Fig. 40 Cysteine modification of RNase A (pdb: 7RSA) via gold-mediated coupling with allenes. ${ }^{420}$

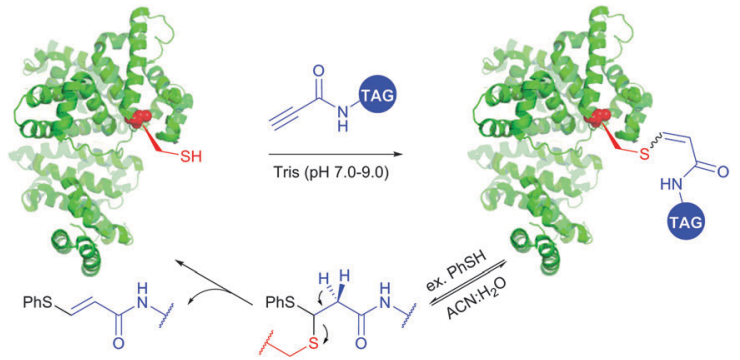

Fig. 41 Cleavable labelling of BSA (pdb: 3V03) with arylalkynone reagents elaborated by Che and associates. ${ }^{417}$

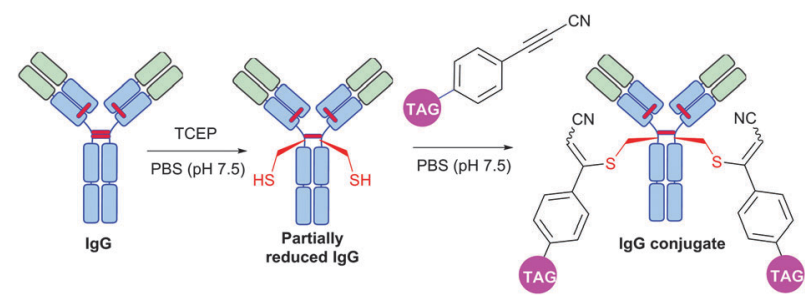

Fig. 42 Preparation of IgG (pdb: 1IGY, red sticks represent four interchain disulfide bonds) conjugates with increased plasma stability using 3-arylpropiolonitriles (APN) described by Wagner and collaborators. ${ }^{429}$

Oxanorbornadienedicarboxylates (OND reagents), strained adducts of furans and electron-deficient alkynes, were found to provide better water stability while retaining selective, rapid, and fluorogenic reactivity towards cysteine compared to corresponding alkynes. ${ }^{430} \alpha, \beta$-Unsaturated ketones and amides (typically acrylamides) can undergo Michael-addition. ${ }^{431,432}$ However, the rate of addition is not generally high enough to provide it with competitive advantages compared with other approaches. Internal Cys residues were reported to accelerate native chemical ligation (see Section 2.3.1), an especially selective approach for N-terminal cysteine conjugation, via cyclic transition states. ${ }^{433-438}$

\subsection{Tryptophan}

Tryptophan (Trp, W) is the second (after cysteine) low abundance amino acid with about $1 \%$ frequency (depending on the living organism), ${ }^{235}$ but approximately $90 \%$ of proteins contain at least one Trp residue in their sequence. ${ }^{439}$ The specific reactivity of tryptophan in proteins is one of the most challenging problems in bioconjugation. In spite of the variety of reagents introduced over the years for selective modification of tryptophan, only a few can be used for conjugation. For instance, such classically used species as Koshland's reagent (2-hydroxy-5-nitrobenzylbromide) ${ }^{440}$ or chlorosulfonium ions ${ }^{441}$ present a high degree of cross reactivity with nucleophilic side chains, nonetheless still being used in numerous studies. These are, for example, investigation of the role of tryptophan in active sites of enzymes, ${ }^{442}$ estimation of its content in proteins, ${ }^{443}$ or determination of the surface accessibility of Trp residues in proteins. ${ }^{444}$

1.4.1 Malondialdehydes. In 2007, further exploring the reactivity of dicarbonyl compounds towards tryptophan described by Teuber and colleagues ${ }^{445} 40$ years earlier, Foettinger et al. ${ }^{446}$ have reported selective reaction of substituted malondialdehydes (MDAs) 

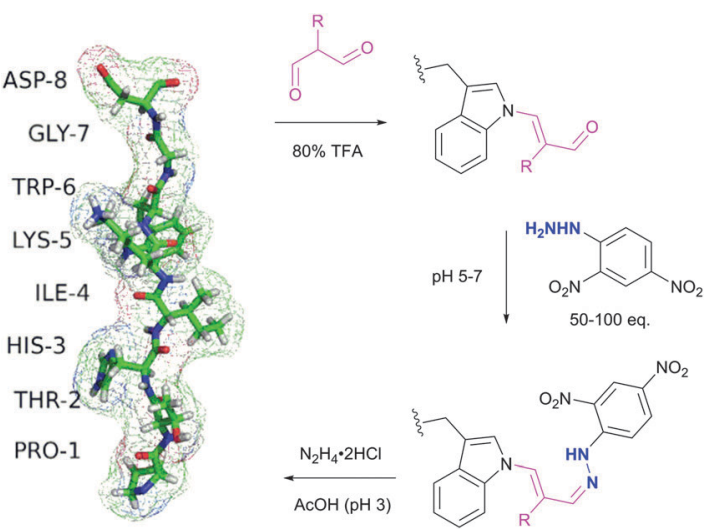

Fig. 43 Selective labelling of Trp side-chain in a 8-mer peptide PTHIKWGD with malondialdehyde described by Foettinger et al. ${ }^{446}$ The peptide structure was simulated using RaptorX web server. ${ }^{447}$

with the indole nitrogen of the Trp side chain of 8-mer peptide PTHIKWGD under acidic conditions (Fig. 43). The obtained substituted acrolein moiety with a remaining reactive aldehyde group can be further converted to a hydrazone using hydrazide compounds, or using other methodologies for aldehyde conjugations. Hydrazine, phenylhydrazine and secondary amines such as pyrrolidine were reported to act as cleavage reagents and allow releasing the free tryptophane after conjugation.

To overpass selectivity issues, namely a known side reaction with Arg side chains, ${ }^{448}$ the conditions for the reactions with MDA, the hydrazone formation and the cleavage of the MDA derivative, had to be optimised concerning $\mathrm{pH}$, buffer, temperature, and reagent. No side reactions of MDAs were observed only under strongly acidic conditions, such as aqueous TFA (80\%). The following hydrazone formation requires approximately 50-100 fold molar reagent excess at a $\mathrm{pH}$ of 5-7 and sometimes increasing the temperature to $50{ }^{\circ} \mathrm{C}$. Although unstable at acidic conditions and when the reagent excess is removed, the hydrazone bond remains firm in alkaline medium ( $\mathrm{pH}>9$ ). The optimal conditions for the cleavage were found using hydrazine (applied as the dihydrochloride salt) in ammonium acetate solution at a $\mathrm{pH} \sim 3$. Demonstrably, these rather rough reaction conditions prevent this methodology from finding widespread use for sensitive protein targets, yet allowing its application in proteomics on peptide digests. ${ }^{449}$

1.4.2 Metallocarbenoids. The same drawback is shared by another approach involving vinyl metallocarbenoids described in 2004 by Francis and collaborators. ${ }^{450}$ The authors have shown, that two Trp residues of horse heart myoglobin can be selectively tagged by a stabilised vinyl diazo compound in the presence of $\mathrm{Rh}_{2}(\mathrm{OAc})_{4}$ (Fig. 44). Difficulties reminiscent to the known instability of the rhodium carbenoid intermediate in aqueous media ${ }^{451}$ were overcome by using an unusual additive - hydroxylamine hydrochloride that was found to facilitate the reaction and enhance efficiency of the tryptophan modification pathway relative to hydrolysis of metallocarbene. However, the use of an excess of the corresponding diazo compound is usually required (at least 4 equivalents) and a 2:3 mixtures of $N$-alkylated versus 2-alkylated products (Fig. 44) are generally obtained in moderate yields. ${ }^{450,451}$

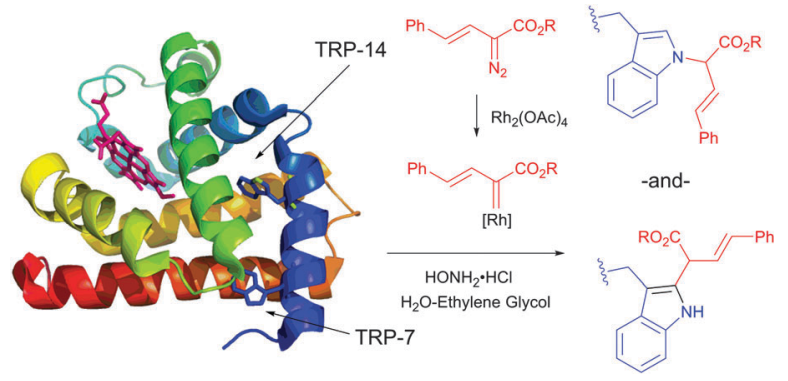

Fig. 44 Modification of horse heart myoglobin (pdb: 1YMB) with rhodium carbenoids described by Francis et al. ${ }^{450}$ A $100 \mu \mathrm{M}$ solution of myoglobin was exposed to stabilised vinyl diazo precursor $(10 \mathrm{mM})$ and $\mathrm{Rh}_{2}(\mathrm{OAc})_{4}$ $(100 \mu \mathrm{M})$ for $7 \mathrm{~h}$. $\mathrm{N}$ - and $\mathrm{C}$-derivatisation of indole rings of both Trp residues - TRP-14 and TRP-7 - were identified by the mass reconstruction. An excess of hydroxylamine hydrochloride $(75 \mathrm{mM})$ is crucial for the efficiency of the conjugation, although its mode of action was not elucidated.

The initially reported reaction conditions tolerated several aqueous solvent systems and proceeded at room temperature. Yet, acidic conditions ( $\mathrm{pH}$ 1.5-3.5) were still necessary for efficient protein labelling and stood out as the main drawback preventing this approach from being generally applicable. For instance, in the same work, authors have stated that myoglobin was denatured and the heme dissociated from the protein due to the high acidity of the medium.

To address these limitations, following efforts of the same group were to improve the $\mathrm{pH}$ range of the tryptophan modification methodology. ${ }^{452}$ For hydroxylamine was found to be ineffective at generating rhodium carbenoids at $\mathrm{pH} \geq 6$, a wide screening of commonly used buffers, as well as additives structurally similar to hydroxylamine $\mathrm{H}_{2} \mathrm{NOH}$, was conducted in order to identify appropriate conditions. From these studies, $t \mathrm{BuNHOH}$ was found to be highly effective at promoting carbenoid addition. Despite the precise mode of action for $t \mathrm{BuNHOH}$ remains unclear, the authors attributed the substantial increase in catalytic activity to a specific interaction between this additive and $\mathrm{Rh}_{2}(\mathrm{OAc})_{4}$. They speculated that, in contrast to hydroxylamine, $t \mathrm{BuNHOH}$ binds to $\mathrm{Rh}_{2}(\mathrm{OAc})_{4}$ through the oxygen, rather than the nitrogen, the latter being disfavoured by the bulky tert-butyl substituent (Fig. 45a), and increases both the stability and the reactivity of the complex at neutral and slightly basic $\mathrm{pH}$.

Interestingly, in the same work, the authors have demonstrated the key role of solvent accessibility of residues in determining the outcome of conjugation on tyrosine using rhodium metallocarbenes. Human FK506 binding protein (FKBP) was identified as a suitable substrate for the study. The only Trp residue (TRP-59) of a wild type FDBP (containing an additional C-terminal threonine residue) is located at the base of the binding pocket, and therefore is unavailable for modification under nondenaturing conditions (Fig. 45b).

To overcome these difficulties, a labelling strategy based on tryptophan mutagenesis followed by chemoselective modification with rhodium carbenoids was utilised. Tryptophan-containing FKBP proteins were expressed in E. coli with C-terminal intein fusions containing a chitin binding domain for affinity 
a)

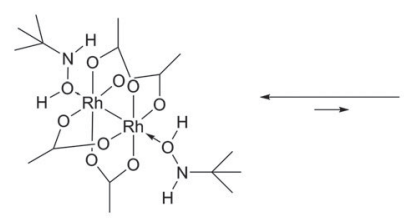

b)

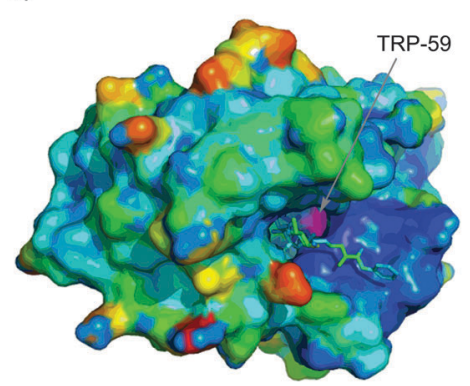

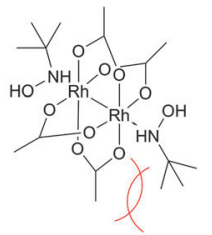

c)

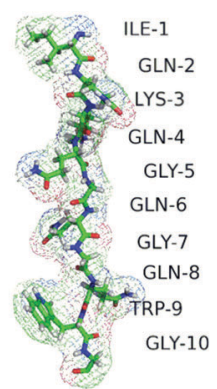

Fig. 45 Optimised metallocarbenoids-based approach, described by Francis and collaborators. ${ }^{452}$ (a) Proposed binding of $t \mathrm{BuNHOH}$ with $\mathrm{Rh}_{2}(\mathrm{OAc})_{4}$ at $\mathrm{pH}$ 6.0. O-Coordination is favoured due to a lower sterical hindrance of tert-butyl groups with acetate ligands. (b) Crystal structure of wild type FKBP (pdb: 1A7X), containing a single, buried tryptophan residue TRP-59 (shown in magenta), which is unavailable for modification under nondenaturating conditions. (c) A peptide tag (IQKQGQGQWG) incorporated into fusion FKBP protein expressed in E. coli as C-terminal intein fusion. The total level of modification was estimated to be in excess of $40 \%$.

purification and short tryptophan-containing peptides (Fig. 45c). Indeed, these newly obtained mutants with solvent accessible Trp residues showed significant level of conjugation (more than $40 \%$ ) under optimised non-denaturating conditions at room temperature.

In 2010, further developing the rhodium-carbenoids methodology for selective tryptophan labelling described 6 years before by Francis et al. ${ }^{453}$ Popp and Ball have reported structure-selective modification of aromatic side chains (expanding its scope to include Tyr and Phe residues) using proximity-driven approach (see Section 5 for details). ${ }^{454}$ Structurally similar to $\mathrm{Rh}_{2}(\mathrm{OAc})_{4}$, metallopeptide complexes with a dirhodium center bounded with two glutamate residues were envisioned to provide delivering of the catalyst to a close proximity of the reactive side chains by exploiting the coiled coil matched peptides, ${ }^{455}$ for molecular peptide-peptide recognition (Fig. 46).

By combining residue-selective chemistry with secondarystructure recognition, the authors have provided a strategy for selective covalent modification of biomolecules. However, only simple diazo reagents without functional handles were used in controlled environments on model peptide substrates.

In the following year, the same group has extended their initial studies to examine the reactivity of whole proteins in a complex, cell-like environment. ${ }^{456}$ For this, the proximity-driven catalysis approach was applied to a recombinant maltose binding protein (MBP), fused with the 21-amino-acid tryptophan-containing coil (almost identical to one used in the initial publication, Fig. 46). Directly after the expression, the lysate was subjected to metallopeptide-catalysed biotinylation. A single band in Western
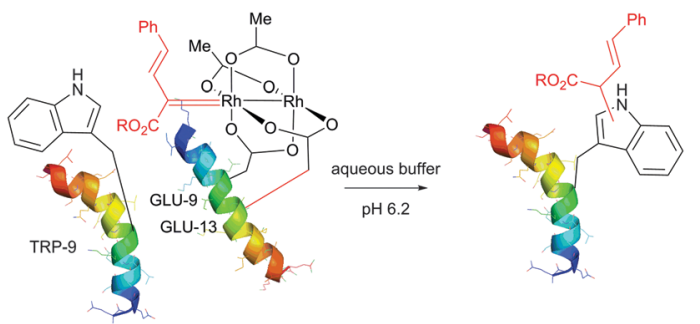

Fig. 46 Selective covalent labelling of the TRP-9 residue of the peptide QEISALEKWISALEQEISALEK with its complementary dirhodium metallopeptide KISALQKQKESALEQKISALKQ described by Popp and Ball. ${ }^{454}$ The rhodium cluster, chelated with two glutamate residues, is brought closer to the reactive Trp residue by peptides coil self-assembling, resulting in selective peptide modification on TRP-9. Peptide structures were simulated using the RaptorX web server. ${ }^{447}$

blot analysis indicated highly selective modification of the modified MBP protein with no nonselective modification to be observed.

\subsection{Histidine}

Histidine (His, $\mathrm{H}$ ) is the only amino acid with a $\mathrm{p} K_{\mathrm{a}}$ in the physiological range, hence often found in active sites of many enzymes and is of crucial importance in mechanisms where abstraction or donation of a proton is needed. Because of its $\mathrm{p} K_{\mathrm{a}}$ value, both the acid and base forms are present at physiological $\mathrm{pH}$ (Fig. 47). Most of the studies on catalytic activity of enzymes and protein-protein interactions involving histidine-containing active centres were done by measuring the influence of site-specific modifications of His residues on the activity of the macromolecule. Main factors influencing histidine reactivity are (1) the $\mathrm{p} K_{\mathrm{a}}$ of the individual His residue, (2) solvent exposure of the residue, and (3) hydrogen bonding of the imidazolium ring. A vast number of studies have been conducted to date, nonetheless, exploiting a small number of chemical functions.

1.5.1 Epoxides. In 2004, Li et al. described two epoxidecontaining fluorescent probes ${ }^{459,460}$ for selective labelling of histidine. A high selectivity of probes towards free histidine in the presence of a 1000-fold excess of other nucleophilic amino acids $^{459}$ and in human serum ${ }^{460}$ (Fig. 48) was achieved after 2 to 3 hours of incubation at $80{ }^{\circ} \mathrm{C}$ in $\mathrm{NaOH}$ solution (pH 11-12).

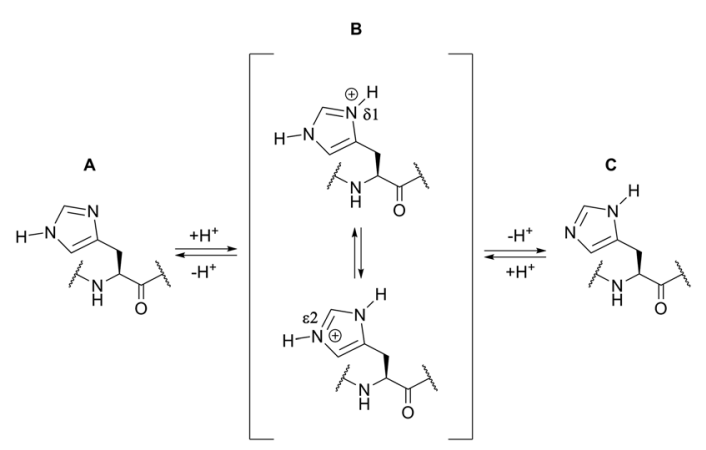

Fig. 47 Tautomerisation equilibrium of the neutral imidazole side chain (base forms A and C) occurring through the acid form B. ${ }^{457}$ Form $A$ is somewhat favoured over $\mathrm{C}$ at neutral and acidic $\mathrm{pH}$, while at basic $\mathrm{pH}$ the form $C$ is preferred. ${ }^{458}$ 

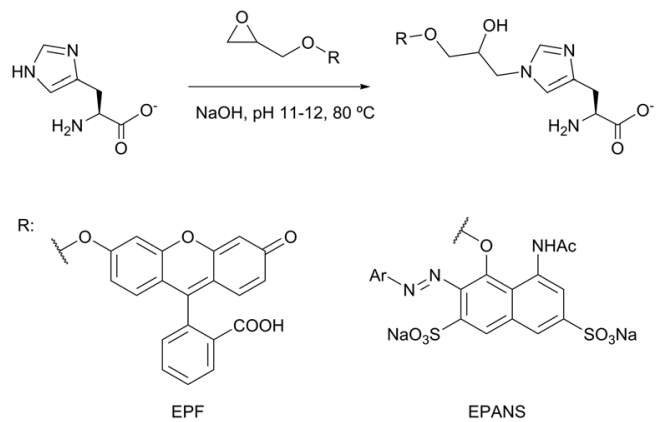

Fig. 48 Fluorescent probes, described by Li et al. for selective ligation of histidine. $^{459,460}$

The authors suggest that these probes can be used for specific labelling of His residues in proteins if a mild reaction condition (lower reaction temperature but longer reaction time) was used, but no example of such application was given. Moreover, considering known reactivity of epoxides with primary amines, thiolates, and hydroxyl groups, such selectivity towards histidine at physiological pH seems improbable. ${ }^{461}$

An affinity-based labelling approach (see Section 4) based on the epoxide opening was developed by Hamachi and collaborators for selective histidine labelling of bovine carbonic anhydrase II. $^{462,463}$ Labelling reagents investigated by the authors must consist of at least three major fragments: (1) a benzenesulfonamide ligand directing specifically to bCA, (2) a reactive electrophilic epoxide for protein labelling, and (3) an exchangeable hydrazone bond between the ligand and the epoxide group for removing the ligand by hydrazone/oxime-exchange and restoring the enzymatic activity (Fig. 49a). Further developing their approach, ${ }^{463}$ the authors added an iodophenyl or acetylene handle on the epoxide-containing fragment to enable the possibility of further derivatisation of the obtained conjugate by Suzuki coupling ${ }^{464}$ or Huisgen cycloaddition $^{465}$ either after or before removing the ligand from the active site of the enzyme (Fig. 49b).

1.5.2 Complexes with transition metals. The affinity of transition metal ions to histidine in aqueous solutions was known for decades. ${ }^{466}$ Copper and nickel ions have the greatest affinity for histidine and this property is the most often used for protein purification by immobilised-metal affinity chromatography (IMAC), exploiting the synergetic coordination effect of oligo-histidine tags (see Section 4). Meanwhile, recently reported by Wang et al., histidine-specific iridium(III)-probe for peptide labelling demonstrates an excellent example of selectivity based on exceptional coordination properties of only one or two His residues (Fig. 50). ${ }^{467}$ Further exploring the advantage of the previously described iridium(III)-complex, used by Wong and colleagues for luminescent labelling of histidine-rich proteins, ${ }^{468}$ authors showed its applicability for histidine labelling in cell-imaging studies.

Although, reckoning obtaining of the coordination complexes to conjugation techniques would be stretching a point, they are however included in this survey because of histidine liability to complexation and increased stability of obtained complexes to decomposition.
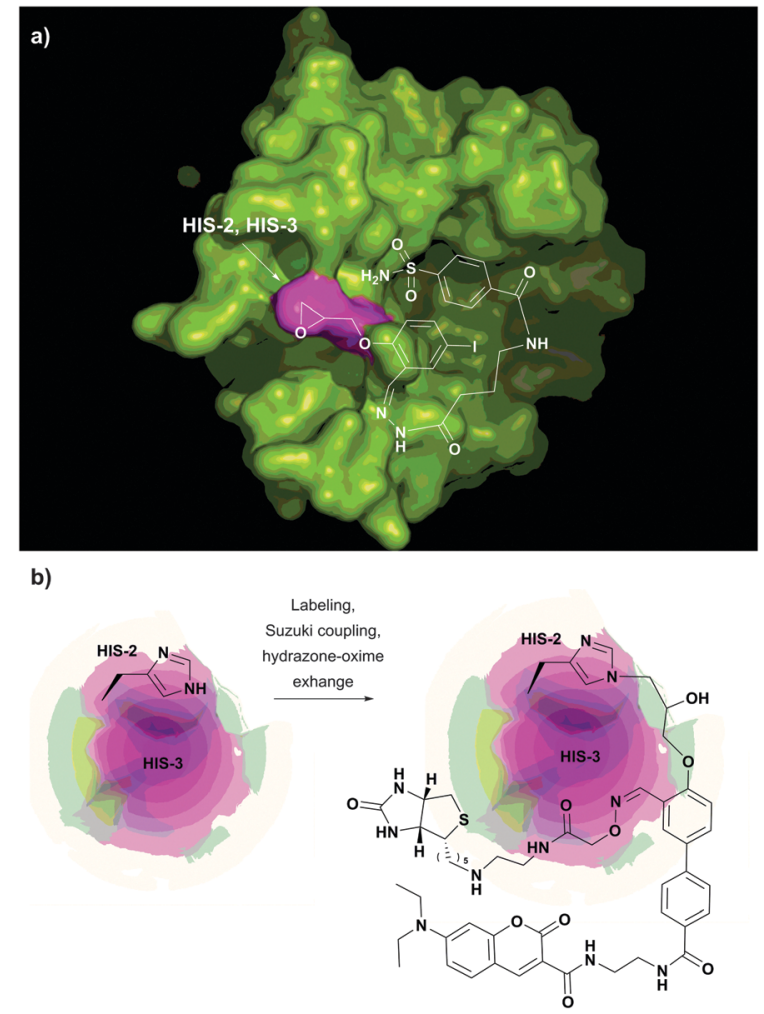

Fig. 49 Affinity labelling, hydrazone-oxime-exchange reaction, and Suzuki coupling reaction on the surface of bCA (pdb: 1V9E) described by Hamachi and collaborators. ${ }^{463}$ (a) Principal structural fragments of the probe: a benzenesulfonamide ligand responsible for targeting bCA; an electrophilic epoxide responsible for reactivity towards vicinal His residues HIS-2 and HIS-3 (shown in magenta), cleavable hydrazone bond, responsible for the recovering of the enzymatic activity; an iodoaryl moiety, utilised for the further bioorthogonal transformation via Suzuki coupling. (b) Dual labelling of bCA in situ: fluorescent labelling of the HIS-2 or HIS-3 alkylated intermediate by Suzuki coupling with a coumarine derivative, followed by biotinylation by hydrazone/oxime-exchange. a)

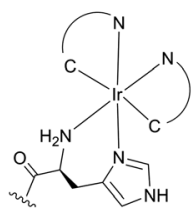

b)

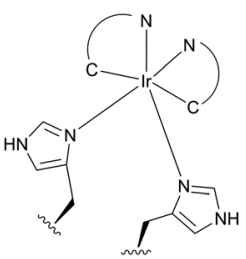

Fig. 50 Luminescent histidine selective peptide tagging. ${ }^{467}$ (a) Labelling of a $\mathrm{N}$-terminal histidine-containing HTat peptide (HRKKRRQRRR). (b) Labelling of a dihistidine motif placed in the middle of a P450dHTat peptide (MLAKGLPPKSVLVKGGHHGRKKRRQRRR).

1.5.3 Michael addition. Several examples of histidineselective Michael addition to the carbon double bond of conjugated aldehydes - 2-alkenals - were found during studies of oxidative modification of proteins. ${ }^{469,470}$ Even though alkenals are known for the modification of the other basic amino acid residues in the protein, ${ }^{471}$ Zamora et al. succeeded in achieving a high level of histidine ligation in bovine albumin by incubation 


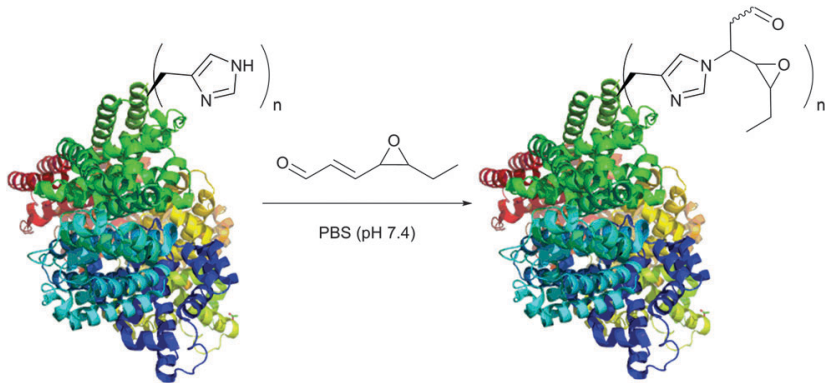

Fig. 51 Michael addition of His residues of bovine albumin (pdb: 3V03) to 4,5-epoxy-2-alkenal described by Zamora et al. 470

in PBS buffer (pH 7.4) at $37{ }^{\circ} \mathrm{C}$ (Fig. 51), however, a reaction of Lys residues was also observed. ${ }^{470}$ Using similar conditions - the incubation in phosphate buffer (pH 7.2) at $37{ }^{\circ} \mathrm{C}$ - Uchida and Stadtman $^{469}$ were able to tag selectively insulin (which contains no sulfhydryl groups) with 4-hydroxynon-2-enal. In both studies, the authors suggest only His residues are modified, but definitive evidence on this point is absent. Obtained conjugates contain active aldehyde residues and represent examples of protein carbonylation, allowing their derivatisation with aldehyde-selective reagents. ${ }^{472}$

1.5.4 Miscellaneous histidine-selective reagents. Some examples of the selective histidine tagging by reagents that, in general, react more avidly with other nucleophilic residues were reported to date. For instance, Pramanik and colleagues achieved dominant PEGylation rh-interferon- $\alpha 2 \mathrm{~A}$ on histidine at mildly acidic levels of $\mathrm{pH}$ with a classic amine PEGylation succinimidyl carbonyl precursor. ${ }^{473}$ Another group reported selective reaction of His residues of D-amino acid oxidase with dansyl chloride in $0.05 \mathrm{M}$ phosphate buffer at $\mathrm{pH}$ 6.6. ${ }^{474}$ These reaction conditions resulted in virtually complete inactivation of the enzyme after the reaction and its complete reactivation after the reaction with $0.5 \mathrm{M}$ hydroxylamine $\left(\mathrm{NH}_{2} \mathrm{OH}\right)$. Such reactivation excludes reaction of primary amino groups, and amino acid analysis suggested that the reaction had not occurred with an oxygen nucleophile such as serine or tyrosine. Even $\alpha$-halo carbonyl compounds - phenacyl bromides, $\alpha$-halo carboxylic acids and amides - classically known for their selectivity towards thiols electrophiles as were found to be histidine selective on several substrates and in carefully tuned conditions. ${ }^{475-479}$

Described by Pauly in the beginning of the 20th century, ${ }^{480,481}$ the reaction with diazonium salts has been only used for colorimetric determining of His residues ${ }^{482}$ and was not further elaborated for bioconjugation. Other classical examples of chemical modification of histidine are mainly reactions with pyrocarbonate, sulfonyl chlorides, sulfonic esters, phenacyl- and acylbromides and activated esters. $^{229}$ These electrophiles react readily with other nucleophilic groups presented in proteins (thiols, amines, alcohols, or guanidino groups) and require a careful tuning of the reaction conditions to achieve sufficient selectivity. For instance, at low pH (generally < 6.0) these reactions are quite selective for histidine, as the main side reaction with the $\varepsilon$-amino group of lysine proceeds very slowly. ${ }^{483}$

Reader should, nonetheless, be aware, that these examples do not represent a general rule, but an exception from it. To avoid the reactions of more nucleophilic functions, the His residue must have been located in a unique microenvironment ${ }^{476,477}$ or have an enhanced nucleophilic character, ${ }^{478}$ but even in this case, prior modifications of highly reactive Cys residues are often inevitable. $^{479}$

\subsection{Tyrosine}

Tyrosine (Tyr, Y) is one of the important amino acid residues which is known to be the active centre in many enzymes (notably tyrosinespecific protein kinases) and is used in signal transduction and cell signalling. ${ }^{484,485}$ Occurring with intermediate to low frequency in native proteins, tyrosine is often considered as an attractive target in bioconjugation, despite being often partially or completely buried due to the amphiphilic nature of the phenolic group.

The reactivity of tyrosyl moiety is easily influenced by its deprotonation, which is a function of the microenvironment inside the protein. All described methodologies take advantage of either the peculiar chemical properties of the electron-rich aromatic ring, or the easiness of the tyrosine hydroxyl group to be transformed into highly reactive phenolate.

1.6.1 $O$-Derivatisation. Although $O$-acetylation of the tyrosyl residue with acetic anhydride and $\mathrm{N}$-acetylimidazole is arguably the most widely used technique for tyrosine modification, ${ }^{229}$ its application for conjugation is rather limited. Mainly, these limitations are due to low selectivity of acylation in the presence of other nucleophilic amino acid residues and modest stability of obtained conjugates.

An elegant approach - the affinity labelling - allows surpassing the selectivity issues of tyrosine acylation by ligand-tethered directing of the reaction. In the case of tyrosine-selective modification, an acyl transfer catalyst is connected to a ligand with a high affinity to the target protein. ${ }^{462,486}$ The acyl group activated by the anchored catalyst is brought to the binging pocket of the protein and transfers an acyl moiety on the nucleophilic Tyr residue in close proximity. Utilizing this methodology, Hamachi et al. ${ }^{486}$ demonstrated selective tagging of Y51 residue of Congerin II (Fig. 52) using a suitable saccharide as a ligand to the target lectin (carbohydrate-binding protein) and DMAP (4-dimethylaminopyridine) as an acyltransfer catalyst. In a similar way, Broo and collaborators ${ }^{487}$ have demonstrated the possibility of a site-specific acylation of a

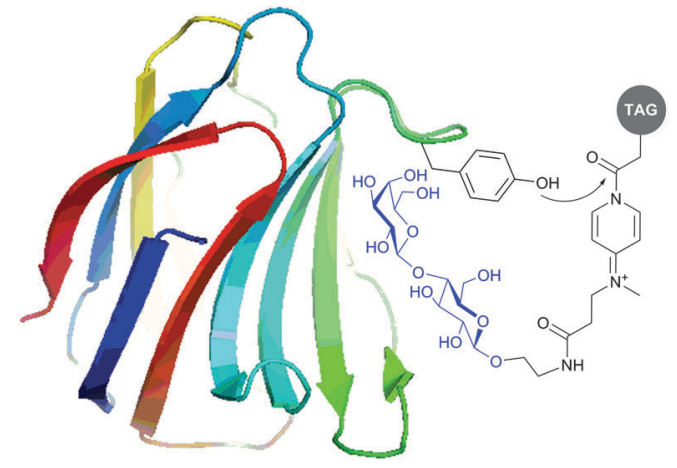

Fig. 52 Sugar-DMAP (4-dimethylaminopyridine) assisted Y51-specific acylation of Congerin II described by Hamachi et al. ${ }^{486}$ Schematic representation is made using lactose-ligated Congerin II crystal structure published by Muramoto et al. ${ }^{488}$ (pdb: 1IS4). 
tyrosine residue situated in an active site of human glutathione transferase (hGST).

Miller and collaborators have shown that biotinylation with NHS esters (see Section 1.1.2) may result in preferential $O$-acylation of hydroxyl-containing residues - serine, threonine and tyrosine (though to a greater extent of the first two) - when they are located two positions next to histidine (i.e. in sequences His-AA-Tyr, where AA refers to any amino acid). ${ }^{85,88}$

Several approaches for labelling involve the initial modification of tyrosine and successive conjugation of an obtained intermediate. For instance, an ortho-nitration of tyrosine with tetranitromethane $(\mathrm{TNM})^{489}$ or peroxynitrite ${ }^{490}$ results in obtaining of $o$-nitrotyrosine that can be then reduced by sodium dithionite $\left(\mathrm{Na}_{2} \mathrm{~S}_{2} \mathrm{O}_{4}\right)$ to form an $o$-aminotyrosine.

Although much less reactive than aliphatic amines at neutral $\mathrm{pH}$, the aromatic amine of $o$-aminotyrosine can selectively react with amine-reactive reagents at lower $\mathrm{pH}^{491,492}$ Namely, Nikov et $a{ }^{492}$ have demonstrated that selective labelling of aminotyrosines is achievable in the presence $\mathrm{N}$-terminal and $\varepsilon$-amino groups of lysines by using NHS-activated ester at particular reaction conditions (acetate buffer, pH 5.0, 2 hours). Exploitation of the $\mathrm{p} K_{\mathrm{a}}$ difference between aminotyrosyl residues and other reactive groups in proteins (4.75 for aminotyrosine, whilst much higher values for N-terminal and side-chain amino groups, see Section 1.1) allows selective labelling thereof. The method was validated on model peptides and then applied to a human serum albumin modification (Fig. 53).

Despite the reaction of TNM and peroxynitrite with proteins being reasonably specific for tyrosine, side reactions with histidine, methionine and tryptophan have been reported, as has oxidation of sulfhydryl groups. The latter would seem to be the most common side reaction, as it can result in disulfide bond formation and the formation of oxidation products such as sulfone and sulfenic acid derivatives. As a general rule, it is normally assumed that the reaction of nitration reagents with Cys residues proceeds equally well at $\mathrm{pH} 6$ and $\mathrm{pH}$ 8, while the reaction with tyrosine occurs at $\mathrm{pH} 8$ and not at $\mathrm{pH} 6$.

In a like manner, the phenol group in Tyr residues can be initially ortho-formylated with chloroform in an alkaline medium to a salicylaldehyde derivative, and then undergo a reaction with ortho-phenylenediamine derivatives to form fluorescent benzimidazoles as conjugation products (Fig. 54). ${ }^{493,494}$

Further exploiting the methodology developed by Trost and Toste for selective $O$ - and $C$-alkylation of phenols with $\pi$-allylpalladium complexes, ${ }^{495,496}$ Francis et al., have demonstrated the possibility of selective allylic alkylation of surface-exposed tyrosines of several full-size proteins. ${ }^{497}$

1.6.2 O-Oxidative coupling. In 1995, Kodadek and co-workers ${ }^{498}$ reported that $\mathrm{Ni}$ (II) complexed with a Gly-Gly-His (GGH) was chemically activated by oxone $\left(\mathrm{KHSO}_{5}\right)$ or magnesium monoperoxyphthalate (MMPP). A Ni(III) oxo intermediate is hypothesised to promote protein cross-linking. ${ }^{499}$ Other peptides, notably $\mathrm{His}_{6}{ }^{500,501}$ or the entire ribonuclease A protein, ${ }^{502}$ which can be incorporated into proteins of interest at the genetic level, have been shown to be effective ligands for nickel catalysed oxidative cross coupling.
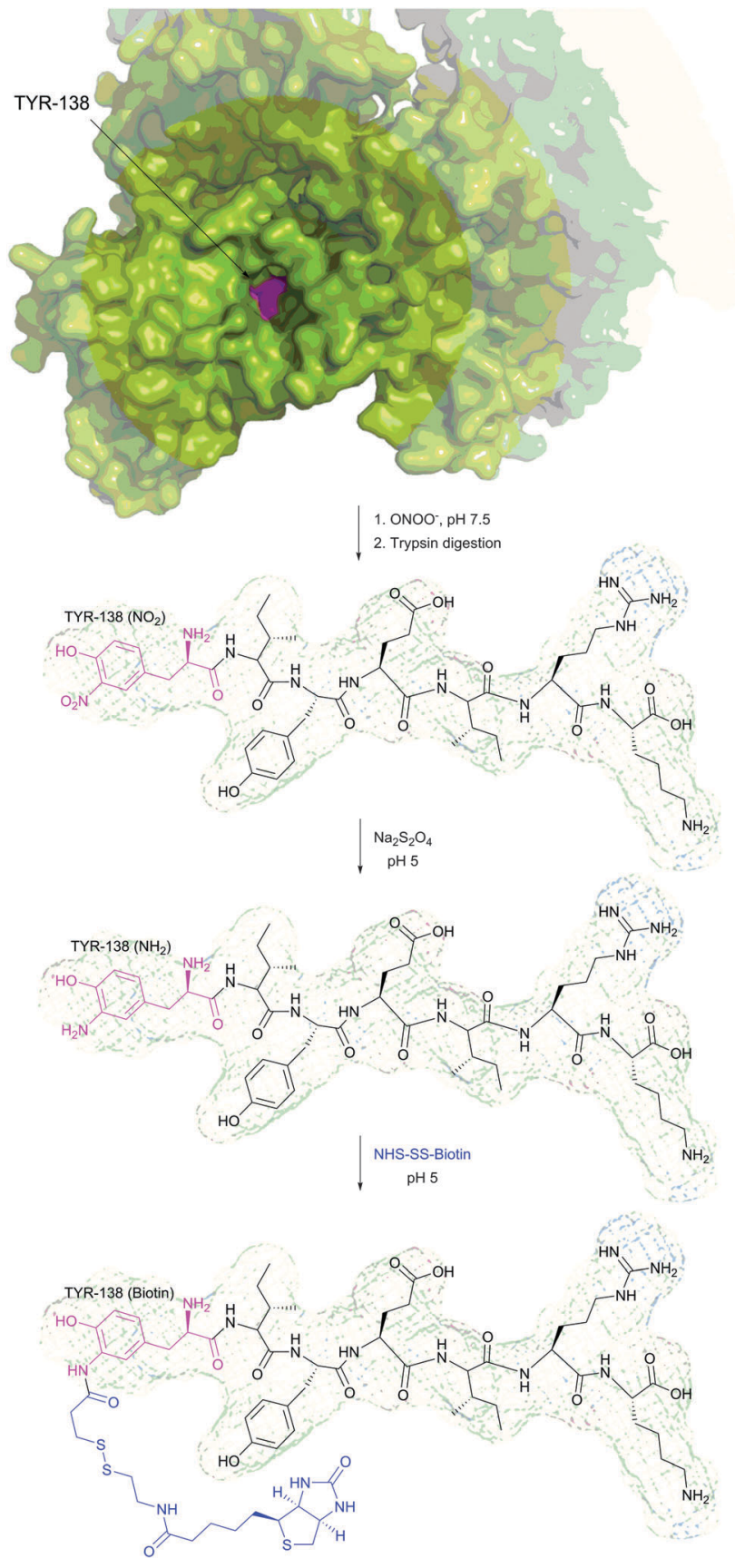

Fig. 53 Two-step biotinylation of HSA (pdb: 1AO6) described by Nikov et al. ${ }^{492}$ Preferential nitration of TYR-138 (shown in magenta) and TYR-411 residues of HSA with peroxynitrite was achieved using protocol described by Jiao and colleagues. ${ }^{490}$ Aminotyrosine of the peptide ${ }^{138}$ YIYEIARK ${ }^{144}$, obtained after the step of reduction with sodium dithionite and following digestion of nitrated HSA with trypsin, were selectively modified with a cleavable biotin-containing reactant at $\mathrm{pH}$ 5.0.

The photoactivable metal-catalysed version of tyrosine oxidation chemistry is significantly faster than the one achieved by Ni(II)peptide complexes. It has been largely exploited by Kodadek and co-workers for cross-linking of closely associated proteins. ${ }^{503-505}$

These coupling reactions are hypothesised to occur through the addition of tyrosyl radicals to adjacent Tyr residues (Fig. 55). It is worth mentioning, that in some cases nearby 


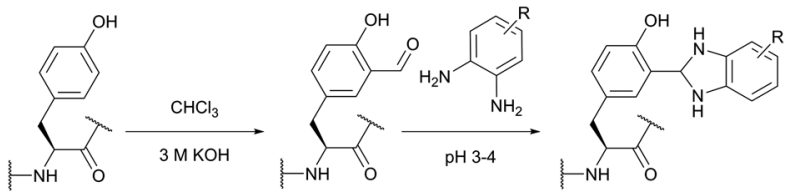

Fig. 54 Two-step tyrosine modification by selective formylation of the tyrosyl residue at the ortho position of its phenolic moiety (reaction with chloroform in potassium hydroxide solution) and further derivatisation of the resulting aldehyde described by Kai et al. and Ishida et al. ${ }^{493,494}$

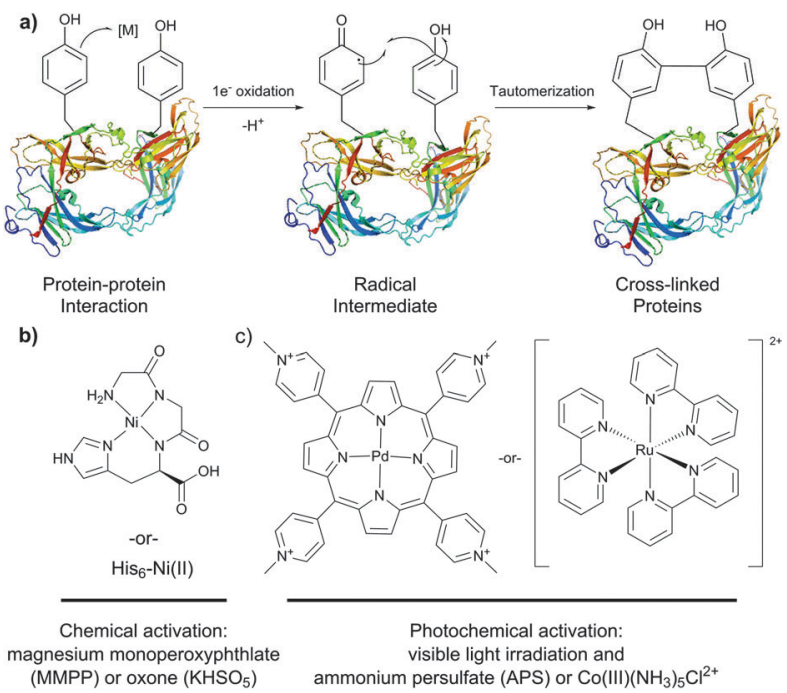

Fig. 55 Transition metal catalysed oxidative cross-linking. (a) Schematic representation of the protein-protein cross-linking, described by Burlingame et al. ${ }^{506}$ The tyrosyl radical generated from ecotin (pdb: 1ECZ) $\mathrm{D} 137 \mathrm{H}$ mutant after the abstraction of an electron with $\mathrm{Ni}(॥)-\mathrm{GGH}$ complex reacts with additional tyrosine residue on nearby protein that are in close proximity due to a significant protein-protein interaction to give a dimer. (b) Chemically activated by MMPP or oxone as stoichiometric oxidants, $\mathrm{Ni}(I)$-peptide complexes can be used as efficient catalysts of cross-linking. (c) Photochemically activated by visible light in the presence of $\left(\mathrm{NH}_{4}\right)_{2} \mathrm{~S}_{2} \mathrm{O}_{8}$ or $\mathrm{Co}(\mathrm{III})\left(\mathrm{NH}_{3}\right)_{5} \mathrm{Cl}_{2}{ }^{+}$as electron acceptors, transition metal complexes are used as catalysts of cross-linking.

tryptophan and other nucleophilic side chains can also participate in oxidation. ${ }^{503}$ For more details, the reader is directed to a review by Bonnafous and a publication of Francis that provide an excellent overview of oxidative cross-linking techniques. ${ }^{507,508}$

The use of cerium(Iv) ammonium nitrate (CAN) - a classical one-electron oxidant - for chemoselective ligation on tyrosine was demonstrated by Francis et al. (Fig. 56). ${ }^{509}$ After the optimisation of reaction conditions, the authors could achieve modification of tyrosine-containing proteins with high yields at neutral $\mathrm{pH}$ and low substrate concentration and applied this strategy to modify both native and introduced residues on proteins with polyethylene glycol (PEG) and small peptides, although dealing with the concurrent reaction of Trp residues. ${ }^{509}$

Notwithstanding the issue of specificity, photo-oxidation and oxidation of techniques of tyrosine ligation continue to be of considerable interest for the study of protein-protein interactions, ${ }^{510}$ mapping multi-protein complexes, ${ }^{511}$ or assembling of macromolecules. ${ }^{512}$

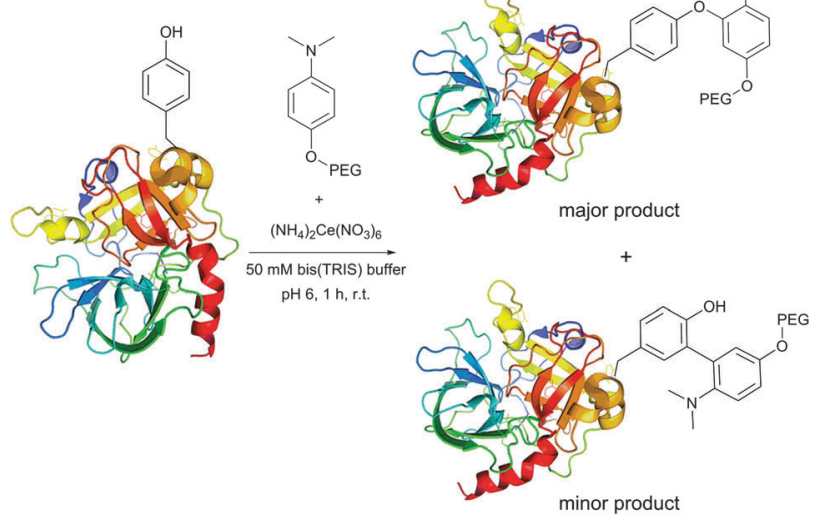

Fig. 56 PEGylation of four solvent-accessible Tyr residues of chymotrypsinogen (pdb: 1EX3) reported by Francis et al. ${ }^{509}$ (only Y171 residue is shown for simplicity reasons). Intermediate tyrosyl radical, generated in the presence of cerium(IV) ammonium nitrate (CAN), gives two addition products with electron-rich aniline derivative $(O-$ is preferred over C-arylation).

1.6.3 Diazonium reagents. Diazonium reaction of tyrosine has been of special interest ever since its introduction by Pauly in $1915 .{ }^{481}$ In 1959, inspired by these pioneering efforts, Higgins and Harrington advanced the use of this methodology and tried applied it to complex proteins. ${ }^{513}$ The authors concluded that the reaction was not confined to the tyrosine and emphasised its competitive nature and strong dependence on the relative concentration of protein and diazonium salt. Moreover, strongly acidic conditions generally required for the preparation of diazonium salts from anilines, ${ }^{514}$ are not compatible with $\mathrm{pH}$-sensitive proteins. Together with a relative instability of diazonium salts and the prerequisite of their preparation just prior to use, these drawbacks prevented this methodology from the widespread use.

The optimised conditions have nonetheless allowed its application for selective modification of tyrosines on the surface of bacteriophage $\mathrm{MS} 2,^{515,516}$ the modification of the tobacco mosaic virus, ${ }^{517}$ and the direct conjugation on proteins. ${ }^{518}$ Francis and co-workers have demonstrated that highly reactive diazonium salts (i.e. containing electron withdrawing groups in their structure) should be utilised in order to achieve efficient Tyr targeting and avoid concurrent reaction with Lys and His residues (see Section 1.5.4) ${ }^{517}$ Recently described by Barbas et al., formylbenzene diazonium hexafluorophosphate reagent ${ }^{519}$ represents an elegant example of a stable ready-to-use reagent for tyrosine labelling and introduction of an aldehyde bioorthogonal tag, capable for future bioorthogonal modifications (Fig. 57).

1.6.4 Mannich-type reaction. Albeit with no control of selectivity, tyrosine conjugation via Mannich-type cross-linking reaction have been first reported by Fraenkel-Conrat and Olcott ${ }^{520}$ and proceeded through the conjugation of tyrosines with imines formed in situ by condensation of lysine amino groups and formaldehyde. The reaction conditions, namely the necessity of using high concentrations of formaldehyde and significant heating, limit the utility of this approach for the vast majority of biological applications. 

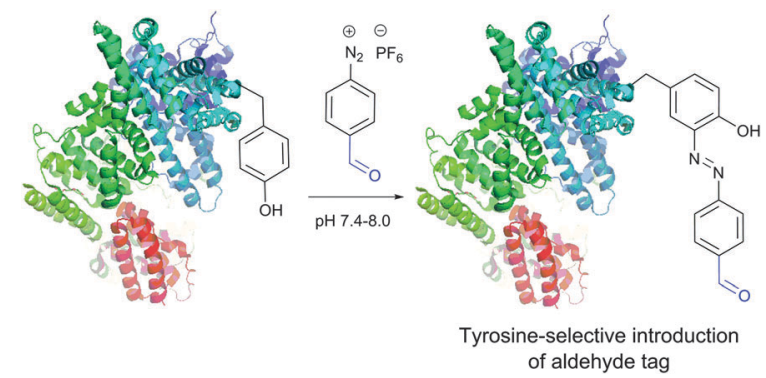

Fig. 57 Selective tyrosine labelling of BSA (pdb: 3V03) by 4-formylbenzene diazonium hexafluorophosphate (FBDP) described by Barbas et al. ${ }^{519}$ for installing a bioorthogonal aldehyde functionality.

The three-component Mannich-type methodology - involving the in situ reaction between a Tyr residue, an amine and formaldehyde was reincarnated more than 50 years later by Francis et al. ${ }^{521}$ The authors demonstrated the possibility of selective modification of tyrosine residues of $\alpha$-chymotrypsinogen A under mild conditions ( $\mathrm{pH} 6.5,25-37^{\circ} \mathrm{C}$ ) and at low concentration of the protein (20-200 $\mu \mathrm{M})$. However, 18 hours of incubation were needed to reach a reasonable level of tagging $(66 \%$ in the case of a fluorescent labelling, Fig. 58). The same group then used this to incorporate synthetic peptides into full-sized proteins. ${ }^{522}$

Despite recognised selectivity issues of a three-component Mannich-type approach for tyrosine labelling, ${ }^{523}$ its main advantage is the possibility to easily vary the participating partners: an aldehyde (Fig. 58, shown in blue) and an aniline residues (Fig. 58, shown in violet). In the following publication on the subject, Francis et al. have demonstrated the viability of NMR-based characterisation of the conjugate isotopically enriched by incorporation of ${ }^{13} C$-formaldehyde into the coupling reaction. ${ }^{523}$ Interestingly, while a reaction by-product arising from tryptophan indole ring was revealed, Cys moiety was found to not participate in the reaction, except in the case of a reduced disulfide, which formed a dithioacetal.

Using similar precursors - electron-rich aniline derivatives Tanaka et al. ${ }^{524}$ could demonstrate the potential of in situ obtained imines as fluorogenic probes for tyrosine labelling. While the educts, as well as the imine derivatives, exhibited

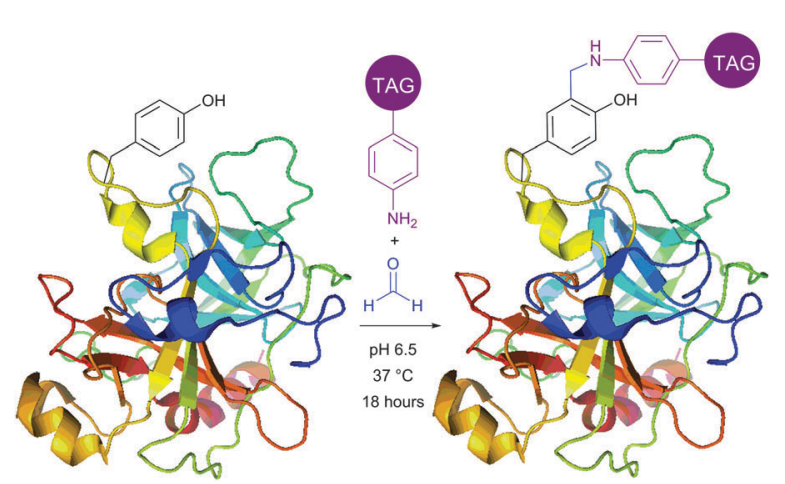

Fig. 58 Three-component Mannich-type selective modification of the Y154 Tyr residue of $\alpha$-chymotrypsinogen $A$ (pdb: 1EX3) by a rhodamine dye, described by Francis et al. ${ }^{521}$ a)

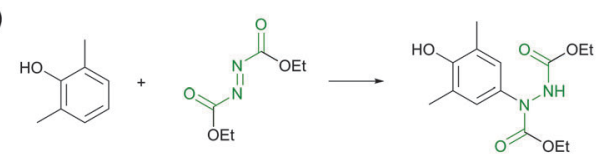

b)
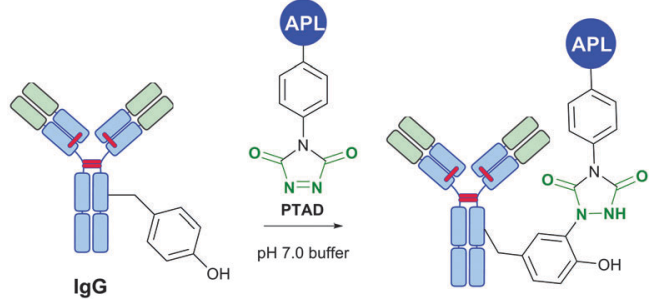

Fig. 59 Reaction of electron-rich arenes with azodicarboxyl compounds (shown in green). (a) First example described by Schroeter in 1969. (b) Conjugation of an HIV entry inhibitor aplaviroc (APL) containing a cyclic diazodicarboxamide derivative - 4-phenyl-3H-1,2,4-triazoline-3,5(4H)dione (PTAD) - with the IgG antibody demonstrated by Barbas. ${ }^{537}$

weak or no fluorescence, the addition products had a significantly higher (more than 100-fold) level of fluorescence.

In the successive study, the same group has expanded this approach to presynthesised cyclic imines completely excluding the need for using an excess of highly reactive formaldehyde. ${ }^{525}$ Although, the authors have clearly demonstrated the applicability of their methodology in water at room temperature over a wide $\mathrm{pH}$ range $(\mathrm{pH} 2-10)$ on a set of model phenols, no example of peptide or protein conjugation has been given.

1.6.5 Dicarboxylates and dicarboxamides. As early as 1969, the reaction of electron-rich arenes with acyclic diazodicarboxylates was reported by Schroeter (Fig. 59a). ${ }^{526}$ Numerous examples of electron-deficient diazodicarboxylates were established in further studies being mainly focused on their synthetic usefulness for electrophilic amination in organic solvents in the presence of activating protic or Lewis acid additives. ${ }^{527-534}$

However, these highly reactive reagents decompose rapidly in aqueous media, which makes them not suitable for bioconjugation. ${ }^{535}$ On the other hand, corresponding diazodicarboxyamide reagents are too stabilised and do not react with phenols in aqueous media. ${ }^{535}$ Cyclic diazodicarboxyamides like 4-phenyl3H-1,2,4-triazoline-3,5(4H)-dione (PTAD) were recently reported by Barbas and collaborators and represent a good compromise between reactivity and stability of diazodicarboxyl-containing reagents. ${ }^{536,537}$ Diazodicarboxylate-mediated tyrosine conjugation is applicable over a wide $\mathrm{pH}$ range, however the highest labeling efficiency was observed at $\mathrm{pH} 7-10 .{ }^{536}$ A versatile class of stable PTAD precursors, possessing different functional groups, was developed and applied for a selective tyrosine conjugation (Fig. 59b). Their utilisation implies prior to use oxidation with 1,3-dibromo-5,5-dimethylhydantoin and the addition of a small amount of TRIS (2-amino-2-hydroxymethyl-propane-1,3-diol) during the step of conjugation. The latter is of crucial importance for the coupling selectivity, for it is hypothesised to serve as a scavenger of a putative isocyanate by-product of the PTAD decomposition, which is promiscuous in labelling.

The non-selective labelling of other aromatic side chains of proteins is the Achilles' heel of the vast majority of approaches 
described for tyrosine labelling. Careful tuning of reaction conditions is important for achieving appropriate levels of selectivity. In some cases where purely chemical distinction of reactivity of amino acid moieties is not feasible, catalysis on the basis of molecular shape rather than local environment can be used to induce selectivity. This concept is routinely exploited by enzymes and allows enabling reactivity that would otherwise be kinetically impossible. In 2010, Popp and Ball used dirhodium metallopeptide catalysts for selective conjugation on tyrosine and tryptophan using the concept of the proximitydriven mechanism (see Section 5). ${ }^{454}$ In the following year, Silverman et al. have demonstrated a DNA-catalysed approach for selective labelling of tyrosine, although only on small peptide substrates. ${ }^{538}$

\subsection{Arginine}

With a $\mathrm{p} K_{\mathrm{a}}$ value above 12 , arginine (Arg, $\mathrm{R}$ ) is mainly presented in its protonated form in acidic, neutral, and even most basic environments. Effective delocalisation of a positive charge between nitrogen lone pairs and the double bond favours the formation of hydrogen bonds ${ }^{539,540}$ and makes the guanidinium side chain of arginine the least acidic cationic group among all 20 natural amino acids (Fig. 60). ${ }^{541}$

However, the $\mathrm{p} K_{\mathrm{a}}$ value of arginine was found to vary significantly in the microenvironments within certain proteins, ${ }^{542,543}$ allowing, in terms of Leitner and Lindner, ${ }^{544}$ the grouping of arginines in "exposed" or "partially buried" residues, basing on the difference of their reactivities.

Most of the described approaches for arginine labelling and modification exploit the chemistry of $\alpha$-dicarbonyl compounds. For instance, introduced by Takahashi ${ }^{545}$ as an arginyl reagent, phenylglyoxal has since been applied for the study of complex systems in the past decade. ${ }^{546-550}$ The reaction occurs under mild conditions and consists of two steps: first addition of phenylglyoxal resulting in the formation of hydrolytically instable imidazolidine diol, and the second step results in a relatively stable addition product (Fig. 61).

Substituted phenylglioxal analogs, such as $p$-hydroxyphenylglyoxal, $p$-nitrophenylglyoxal and 4-hydroxy-3-nitrophenylglyoxal, p-azidophenylglyoxal (APG) were reported for spectrophotometric and cross-linking study of the modification of arginine in proteins. ${ }^{551-555}$ None of these linkers have however been used in bond-forming conjugation. Because phenylglyoxal, like glyoxal, reacts with $\varepsilon$-amino groups at a significant rate, ${ }^{545}$ many efforts were made to increase its selectivity towards guanidinium residue. Cheung and Fonda have studied the effect of buffers

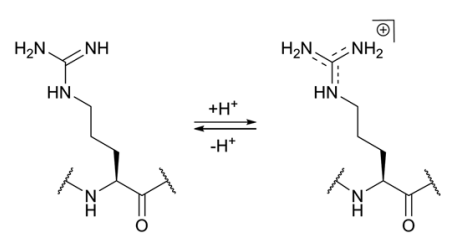

Fig. 60 Protonation of the arginine side chain at neutral pH.

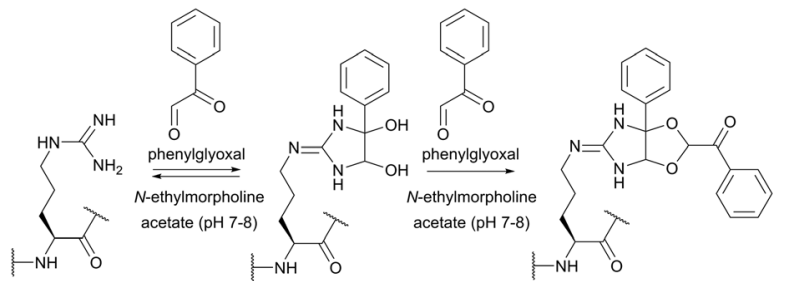

Fig. 61 Reaction of phenylglyoxal with the arginine moiety (reaction condition: $0.2 \mathrm{M} \mathrm{N}$-ethylmorpholine acetate buffer, $\mathrm{pH} 7-8$ ).

and $\mathrm{pH}$ on the reaction rate ${ }^{556}$ and found that the reaction of arginine is greatly accelerated in bicarbonate-carbonate buffer systems, possibly due to the stabilisation of the obtained diol.

Geminal diones - namely 2,3-butanedione (introduced by Yankeelov) ${ }^{557,558}$ and 1,2-cyclohexanedione (introduced by Itano $)^{559}$ - are another well-characterised reagents for the modification of Arg residues. The reaction progresses through the pathway that is similar to the phenylglyoxal addition. However, it was not until the observation that borate had a significant effect on the selectivity of the reaction that the use of this reagents became practical. ${ }^{560,561}$ The presence of borate in the solution allows shifting of the equilibrium during the addition to a guanidine moiety through the stabilisation of reversibly obtained diol (Fig. 62).

In 2005, using this approach, Lindner and colleagues have described a method for the selection of arginine-containing peptides from a tryptic digest of the model proteins (BSA, lysozyme, ovalbumin) by a solid phase capture and release. ${ }^{562}$ First, arginine containing peptides presented in the digest were covalently modified on the guanidine moiety with 2,3-butanedione and phenylboronic acid under alkaline conditions. Polymeric materials allowing the immobilisation of phenylboronic acid were then used to capture the arginine-peptides on a solid support while washing away all not covalently bonded arginine-free peptides. Finally, the arginine-peptides were cleaved again from the boronic acid beads due to the reversibility of the reaction. Photoactivable bifunctional reagents for cross-linking of arginine moieties have been elaborated by Ngo et al. and Politz et al. to study enzymes with an arginine at their active sites. ${ }^{555,563}$

Arginine-specific PEGylation of lysozyme using polyethylene glycols containing an $\alpha$-oxo-aldehyde motif in borate buffer was recently reported by Gauthier and Klok ${ }^{564}$ and represents mild and selective method for protein modification (Fig. 63). Other methods described to date ${ }^{565,566}$ possess selectivities, which are not sufficient (especially in the presence of Lys moieties) to consider them as suitable for bioconjugation.

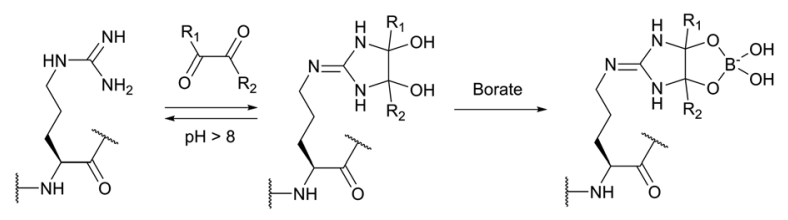

Fig. 62 Reaction of 2,3-butanedione with arginine residues of Carboxypeptidaze $\mathrm{A}$ (pdb: $1 \mathrm{HDU}$ ) in the presence of borate described by Riordan. ${ }^{560}$ 


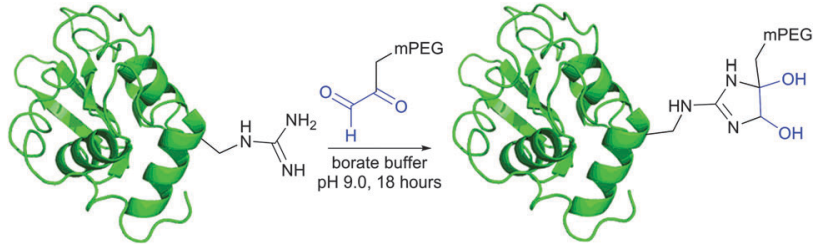

Fig. 63 Selective PEGylation of lysozyme's arginine side chains (pdb: 2LYZ) described by Gauthier and Klok. ${ }^{564}$

\subsection{Aspartic and glutamic acids}

Carboxylic acid groups can be found in protein structure either on its C-terminus, or as side chains of Asp and Glu. Due to the low reactivity of carboxylate in water, it is usually difficult to selectively conjugate proteins at these moieties. Carboxylic acid should thus generally be converted to a more reactive ester by means of so called activating reagents.

For more than a half century, carbodiimide-mediated activation is the most extensively used methodology for the modification of free-acids in protein. ${ }^{567,568}$ The reaction of carbodiimides with protonated carboxyl groups yields activated acylisoureas, which then react smoothly with a variety of nucleophiles, namely amines (Fig. 64). ${ }^{569}$ It is important to utilise weakly basic amines, that remain deprotonated and thus reactive at $\mathrm{pH}$ below 8.0, to avoid protein cross-linking occurring at higher $\mathrm{pH}$ values. For this reason, weakly basic hydrazides are often reagents of choice in coupling reactions with activated carboxylic acids. ${ }^{570}$ Although waterinsoluble carbodiimides (DCC, DIC) still continue to be useful for acid-selective protein conjugation, ${ }^{571,572}$ most current reports exploit water-soluble carbodiimides such as 1-ethyl-3-(3-dimethylaminopropyl)carbodiimide (EDC). Developed by Sheehan and Hlavka $^{573,574}$ these carbodiimides first proved their especial usefulness as zero-length cross-linking reagents to study proteins. ${ }^{574}$ Subsequent studies were devoted for the application of carbodiimides for quantitation of accessible carboxyl groups in proteins, ${ }^{567,568,575}$ preparation of antigenic conjugates,${ }^{576}$ and protein immobilisation. ${ }^{577}$

As mentioned previously, the upper limit for the optimal $\mathrm{pH}$ of carboxylate conjugation is defined by the reactivity of free amino groups present in protein. The lower limit is mainly determined by aqueous stability of the activating reagent. Borders and co-workers ${ }^{578}$ studied the stability of EDC in aqueous solution.
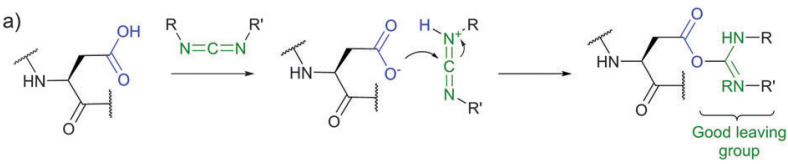

b)
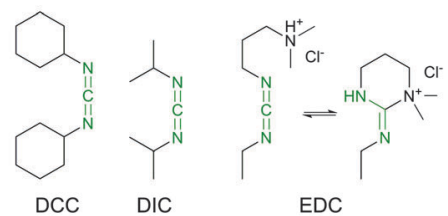

EDC

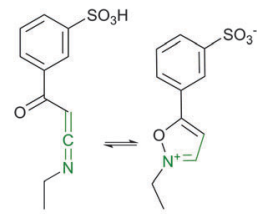

Woodward's reagent $\mathrm{K}$
Fig. 64 (a) Carbodiimide-mediated activation of the carboxylic acid side chain of glutamate. (b) Relevant examples of activating reagents.
It was found that EDC has a $T_{12}$ of 37 hours (pH 7.0), 20 hours (pH 6.0), and 3.9 hours (pH 5.0) in $50 \mathrm{mM}$ MES buffer at $25^{\circ} \mathrm{C}$; in the presence of $100 \mathrm{mM}$ glycine, the $T_{12}$ values were 15.8 hours, 6.7 hours, and 0.73 hours respectively. This supports the optimal $\mathrm{pH}$ for acid-selective conjugation to be in a range from 6.0 to 7.0. NHS (or its water-soluble analogue sulfoNHS) is often included in coupling protocols to improve efficiency or to create a more stable intermediate. Possible side-reactions involving activating reagents were recently reviewed by Valeur and Bradley. ${ }^{579}$

Woodward's reagent $\mathrm{K}$ (N-ethyl-5-phenylisoxazolium-3'sulfonate $)^{580}$ and analogous substrates were used as activating reagents of carboxyl groups for synthetic purposes. Bodlaender et al. ${ }^{581}$ used $N$-ethyl-5-phenylisoxazolium-3-sulfonate, the $N$-alkyl derivatives of 5-phenylisoxazolium fluoroborate, to activate carboxyl groups on trypsin for subsequent modification with methylamine or ethylamine.

Lastly, several studies revealed unexpected examples of carboxyl group modification with reagents usually reacting far more effectively with other nucleophiles. For instance, $p$-bromophenacyls and iodoacetamides have been found to selectively alkylate carboxylic acid moieties of pepsin and ribonuclease T1 respectively. ${ }^{582-584}$ However, the applicability of these reagents is not general and is appropriate on specific substrates only.

\subsection{Methionine}

In spite being often considered a rather simple target for chemical modification (mainly through the oxidation and the reaction with $\alpha$-halo acetic acids and their derivatives), ${ }^{585,586}$ only a handful of conjugation methodologies involving methionine (Met, M) were described up to the present.

All approaches described in literature exploit alkylation of Met residues in acidic media. Although many nucleophilic functional groups present in proteins can react with alkylating reagents, at low $\mathrm{pH}$ all of them except methionine exist in protonated forms, which greatly decreases their reactivity. ${ }^{587}$ Consequently, alkylations of other nucleophilic functional groups, such as thiols, are commonly conducted at high $\mathrm{pH},{ }^{400}$ while methionine is the only functional group in proteins able to react with alkylating reagents at low $\mathrm{pH}$.

Basing their research on pioneering studies being done by Toennies in the $1940 \mathrm{~s},{ }^{589,590}$ Kramer and Deming have recently reported a reversible chemoselective labelling of methionine in peptides and polypeptides. ${ }^{588}$ Treatment of the model peptide (PHCRKM) with alkylating reagents of different structures in $0.2 \mathrm{M}$ aqueous formic acid ( $\mathrm{pH} 2.4$ ) gave a single product, where only the Met residue was alkylated. This resulting sulfonium salts can readily be dealkylated by addition of pyridine-2 $(1 H)$-thione (PyS) to give the starting peptide as the sole product along with the alkylated PyS byproduct. The removal reaction was found to be selective and allows selective dealkylating using concentrations of PyS that do not react with the disulfide bond in cystine under identical conditions (Fig. 65). 


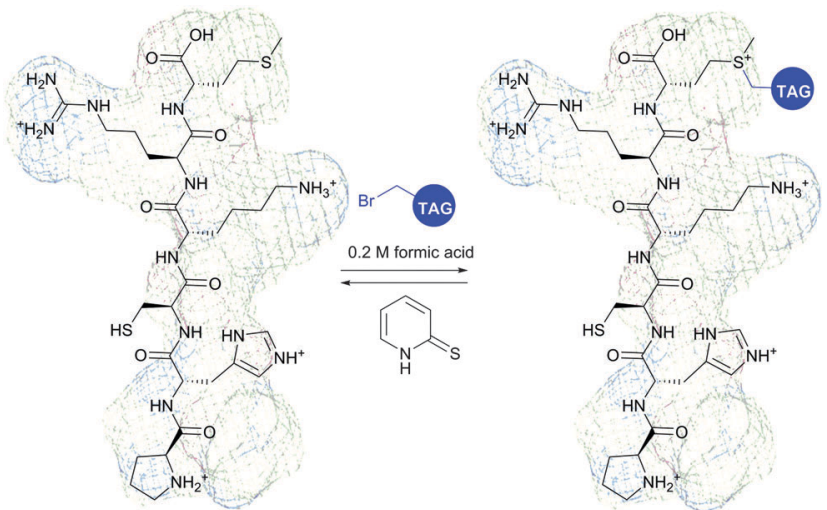

Fig. 65 Selective reversible modification of a 6 -amino-acid peptide (PHCRKM) via methionine alkylation reported by Kramer and Deming. ${ }^{588}$ No reactions with other amino acids were detected.

\section{N-terminal conjugation}

\section{$2.1 \alpha$-Amino groups}

The most important thing to note about N-terminal amino groups is that these are the only primary amines present in the protein structure that possess an adjacent amide bond in $\alpha$-position, which slightly influences their reactivity. Consequently, the majority of classical methodologies exploit this peculiarity of the N-terminus.

2.1.1 Classical approaches. As rather unpolarizable nucleophiles, amines react preferentially with hard electrophiles like acid anhydrides and acyl halogenides. ${ }^{591}$ The anchimeric influence of the adjacent amide bond consists in the lowering the $\mathrm{p} K$ value of the amine by electron withdrawal. Consequently, this makes the discerning of $\mathrm{N}$-terminal amino groups from the $\varepsilon$-amino groups of Lys residues achievable by working at a $\mathrm{pH}$ close to their $\mathrm{p} K$ values, i.e. under slightly acidic conditions. That is, virtually all methodologies described in the Section 1.1 of this review are, to some extent, applicable for selective labelling of N-terminal amino groups of the proteins.

In the classical work on acetylation of the growth hormone, Reid $^{592}$ has for the first time demonstrated the possibility of selective modification of $\mathrm{N}$-termini, if acetylation is performed with a relatively small amount of acetic anhydride. Further development of this approach has in several protocols for selective labelling of $\alpha$-amino groups of proteins, ${ }^{3,593}$ peptides, ${ }^{594,595}$ and proteomes. ${ }^{596-598}$ Under optimal reaction conditions, the use of a 5 -fold excess of amine-reactive reagent in PBS (pH 6.5) at $4{ }^{\circ} \mathrm{C}$, high levels of selectivity can be achieve after 2-24 hours of reaction. However, the preference for terminal amino groups achieved by control of $\mathrm{pH}$ is rather limited, mainly owing to the fickleness of the $\mathrm{p} K_{\mathrm{a}}$ of the amino group depending on the microenvironment and reaction conditions. Consequently, more proficient methods that rely upon increased chelating ability of N-termini, ${ }^{599}$ direct participation of the adjacent side chains or peptide bond, ${ }^{600,601}$ were developed and represent to date preferential approaches for bioconjugation.

2.1.2 Ketene-mediated conjugation. A method for selective $\mathrm{N}$-terminal modification of proteins by ketenes was introduces by Che and co-workers ${ }^{602}$ and consisted in $\mathrm{N}$-terminal ligation
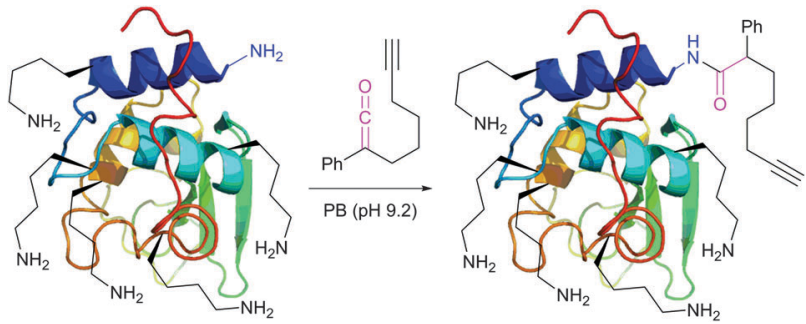

Fig. 66 Selective labelling of lysozyme (pdb: 2LYZ) described by Che. ${ }^{603}$ Moderate-to-high level of selectivity towards $\mathrm{N}$-terminal residue (K1) was achieved in the presence of 5 other in-chain lysines (LYS-13, LYS33, LYS-96, LYS-97 and LYS-116). The ketene was synthesised from the corresponding acid by a two-step protocol: transformation into a mixed anhydride (oxalyl chloride, DCM) followed by the transformation thereof into ketene upon the reaction with a base (TEA, THF).

of peptides through oxidative amide bond formation using the "[ $\left.\mathrm{Mn}\left(2,6-\mathrm{Cl}_{2} \mathrm{TPP}\right) \mathrm{Cl}\right] /$ alkyne $/ \mathrm{H}_{2} \mathrm{O}_{2}$ " systems. Initially, the method was tested on a set of six peptides and demonstrated its applicability. However, inevitable oxidation at Cys and Met residues hindered the application of the protocol in the field of bioconjugation. Only after having conducted the mechanistic studies of this approach, the authors have realised that ketenes generated in situ were the key intermediates accounting for the reactivity; therefore prior preparation thereof would allow for refraining from the need to use an oxidant. In the publication to follow, Che ${ }^{603}$ has introduced a general approach for the modification of $\mathrm{N}$-terminal $\alpha$-amino groups of a series of proteins and peptides using an isolated alkyne-functionalised ketene (Fig. 66). Interestingly, in contrast to classical approaches, increasing the $\mathrm{pH}$ of the reaction mixture did not significantly affect N-terminal selectivity of conjugation. For comparison, the ketene reagent was side-by-side benchmarked with a corresponding NHS-ester. Remarkably, much poorer N-terminal selectivity was obtained for all studied peptide substrates when the NHS ester was used. The reason for this impressive specificity of ketenes, however, remains unelucidated.

2.1.3 Transamination. It was after the success of the conversion of glyoxyloyl groups into glycyl groups (see Fig. 75) ${ }^{604}$ that Dixon and co-workers realised that if a terminal Gly residue can be made by transamination then a terminal residue of any kind might be transformed into a corresponding carbonyl-containing residue by transamination. These introduced carbonyl groups are not naturally occurring functionalities in proteins and can therefore be used as unique loci of attachment for synthetic groups through the formation of hydrazone or stable oxime bonds. ${ }^{605,606}$

Inspired by the pioneering works of Metzler and Snell, ${ }^{607}$ and Cennamo and collaborators ${ }^{608,609}$ on the transamination of simple amino acids and peptides under harsh conditions (heating at $100{ }^{\circ} \mathrm{C}$ and $\mathrm{pH}$ 5.0), Dixon and Moret ${ }^{610,611}$ developed a method for mild transamination in the presence of copper(II) salts, which allowed the reaction to pass at room temperature (Fig. 67). The isomerisation of the imine generated in situ by catalysed 1,3-proton shift transfer is the key step of the transformation which defines both its direction and the reaction rate. It is clearly the activation of $\alpha$-protons of $\mathrm{N}$-terminus by the 


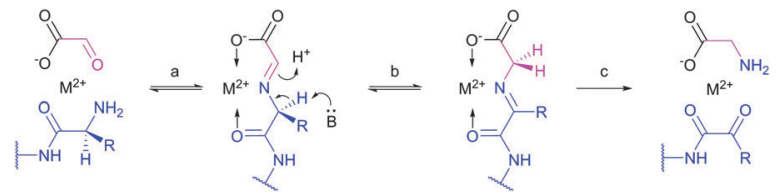

Fig. 67 General scheme of the transamination reaction activated by metal ions (typically $\mathrm{Cu}^{2+}$ and $\left.\mathrm{Ni}^{2+}\right) .{ }^{610}$ Principal steps of the reaction mechanism: (a) generation of the imine; (b) isomerisation of the obtained imine by proton removal; (c) hydrolysis of isomeric imine to generate transaminated reaction partners.

adjacent peptide bond and the metal ions that makes this reaction specific to $\alpha$-amino groups. Interestingly, Dixon reports that no traces of the reaction of lysine side chains were ever observed. ${ }^{600}$

Quite a wide range of reaction conditions has been tested ever since. The discovery that pyridine ${ }^{612}$ and acetate ${ }^{613}$ greatly accelerated the transamination of amino acids led to a slightly milder reaction conditions, which however were still too harsh to maintain the folded structure of most proteins, and were therefore more appropriate for sequence-analysis applications. ${ }^{614}$

Only recently Francis and co-workers ${ }^{601}$ have re-examined Cennamo's approach ${ }^{608}$ of amino acid transamination in the presence of pyridoxal-5-phosphate (PLP, vitamin B6) at $100{ }^{\circ} \mathrm{C}$ and found much milder the reaction conditions when modifying the N-terminal residues of peptides $\left(65^{\circ} \mathrm{C}\right.$ for reaction to be over in 2 hours; $25^{\circ} \mathrm{C}$ to achieve a complete conversion in 24 hours). Screening experiments on different $\mathrm{N}$-terminal amino acids of the peptides indicated that the aldehyde structure strongly influenced the reaction efficiency. Amusingly, known for more than 50 years PLP emerged as the most effective aldehyde among dozens being screened, affording the highest yields at milder reaction conditions.

The mechanism of PLP-catalysed transamination is depicted in Fig. 68. The reaction of PLP and N-terminal amine results in forming of a Schiff base aldimine (a). Then the $\alpha$-proton the

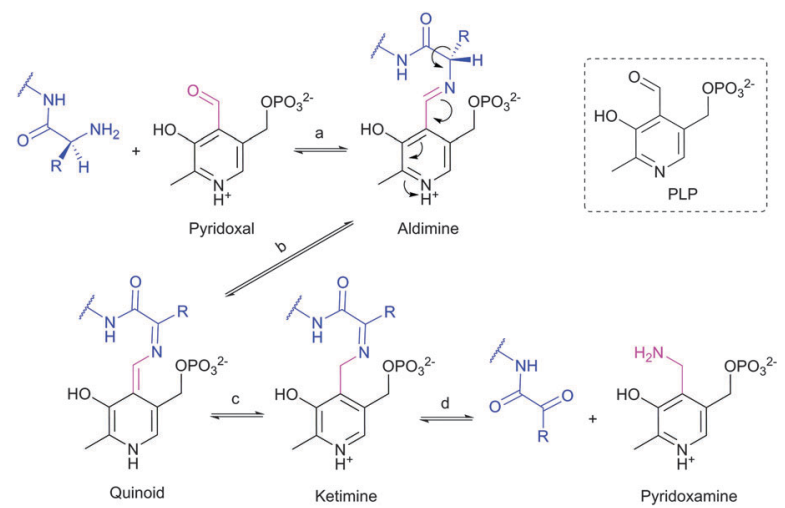

Fig. 68 Structure of PLP and mechanism of PLP-mediated transamination. Reaction pathway consists of (a) condensation reaction between pyridoxal and the amine; ( $b$ and $c$ ) tautomerisation of the obtained aldimine being favourable because of a much lower intrinsic $p K_{a}$ values of $\alpha$-proton (shown in blue); (d) hydrolysis of the resulting ketimine, accompanied by decarboxylation in the case of aspartic acid $(\mathrm{R}=$ $\left.-\mathrm{CH}_{2} \mathrm{COOH}\right)^{601}$

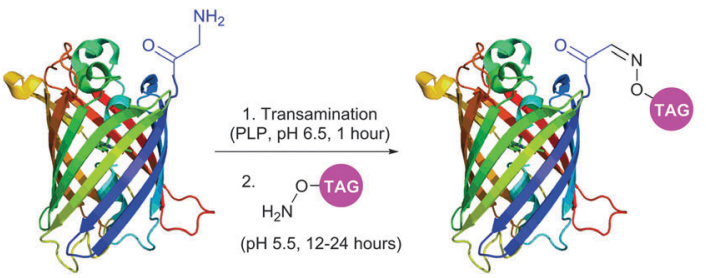

Fig. 69 Site-specific N-terminal labelling of EGFP (pdb: 2YOG). Proteins possessing $\mathrm{N}$-terminal carbonyl groups obtained by in the first step PLPmediated transamination were labelled with hydroxylamine probes in the second step. ${ }^{601}$

amino acid transferred to the $4^{\prime}$ position of the pyridoxal unit (b and c). Finally, hydrolysis of the obtained ketimine leads to the desired $\alpha$-ketoacid and pyridoxamine phosphate (d). Quinoid is an important intermediate for the transformation of aldimine to ketimine and can be found in all transamination reagents described to date.

Under optimal reaction conditions: $10-50 \mu \mathrm{M}$ protein and 10-50 mM PLP at $37^{\circ} \mathrm{C}$ in PB (pH 6-7), a complete conversion is generally achieved after $2-24$ hours. The resulting keto-proteins are generally rather stable and can be concentrated, stored, or lyophylised without any specific precautions. ${ }^{615}$ An example of transamination-conjugation methodology was demonstrated by Francis in the initial publication on selective labelling of an N-terminal glycine residue of horse heart myoglobin and enhanced green fluorescent protein (EGFP, Fig. 69). ${ }^{601}$

Although the side chain of the N-terminus does not participate directly in the transamination mechanism, the reaction rates were found to vary significantly depending on the amino acid in the $\mathrm{N}$-terminal position. ${ }^{616}$ Generally, the majority N-terminal amino acids provide high yields of the desired transaminated products; however, some residues (His, Trp, Lys, and Pro) generate adducts with PLP itself, while other are incompatible with the technique because of known side reactions (Ser, Thr, Cys and Trp) or complete inertness (Pro).

In the attempts for the investigation of transamination reaction scope and limitations, Francis and collaborators have prepared an 8000-member one-bead-one-sequence combinatorial peptide library in which the three $\mathrm{N}$-terminal residues were varied. ${ }^{617}$ Interestingly, the Ala-Lys (AK) motif was found to favour especially the transamination yields (the Lys residue is hypothesised to accelerate the isomerisation step of 1,3-proton shift acting as a general base). To demonstrate this, labelling of the Type III "Antifreeze" Protein and its mutant presenting the AKT sequence on the N-terminus were side-by-side benchmarked. At every time point analysed, the AKT terminus outperformed the wild-type one (GNQ) at different concentrations of PLP.

Although mild reaction conditions of PLP-mediated transamination render it amenable for the modification of intact proteins, ${ }^{618}$ the yields are generally not high and elevated temperatures are usually required, which largely limits the practical applicability of the approach. Given this situation, Francis and co-workers ${ }^{619}$ have utilised above-described combinatorial approach to identify another transaminative agents. As a result, $N$-methylpyridinium-4-carboxaldehyde benzenesulfonate 


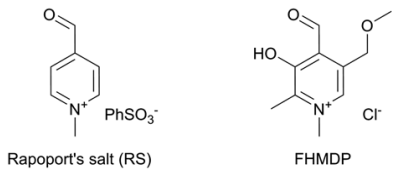

Fig. 70 PLP-analogues used as transamination agents: Rapoport's salt ${ }^{619}$ and FHMDP. ${ }^{621}$

salt (Rapoport's salt, RS, Fig. 70) was identified as a highly effective alternative to PLP. Furthermore, this was found to be particularly efficient for glutamate-rich sequences. ${ }^{619,620}$ The fact that several antibody isotypes dispose at least one glutamate-terminal chain makes RS particularly amenable for their selective conjugation. Remarkably, the difference of transamination reaction rates on Glu- and non-Glu polypeptides was significant enough for selective labelling of only the heavy chain of immunoglobulin G1 (containing the N-terminal Glu residue), while leaving unmodified the light chain. This was assigned to be due to the higher steric hindrance of the already less-reactive substrate ( $\mathrm{N}$-terminal Asp). To ensure these results an IgG1 mutant possessing Asp-Asp-Ser sequence on both chains was prepared. Indeed, this underwent the modification of both sites when exposed to RS. Facing another recognised drawback of Francis methodology, namely low efficiency for bulky amino acid termini (Leu, Ile, Val), Zhang et al. ${ }^{621}$ have elaborated an efficient PLP analogue, FHMDP (Fig. 70), that demonstrated much higher efficiency in transamination thereof.

The above-mentioned transformations provide just a few examples of the rapidly growing field of transaminative modification of proteins. Recent advances have also resulted in elaboration of general approaches for protein immobilisation (Fig. 71), ${ }^{15,622}$ dual fluorescent modification of periplasmic solute binding proteins, ${ }^{623}$ protein PEGylation and PEG-like conjugation (e.g. OEGMAtion), ${ }^{624}$ preparation of phage conjugates, ${ }^{625,626} \mathrm{~N}$-terminus proteomics, ${ }^{627,628}$ enabling Wittig ${ }^{629}$ and Pictet-Spengler ligation on transaminated proteins. ${ }^{630,631}$

2.1.4 2-Pyridinecarboxyaldehydes (2PCA). A promising approach for one-step $\mathrm{N}$-terminal selective modification of proteins using 2-pyridinecarboxyaldehyde (2PCA) derivatives was recently reported by Francis and co-workers. ${ }^{632}$ Because of its structural similarity to pyridoxal-5-phosphate and Rapoport's salt, 2PCA was occasionally discovered by the authors during a screening of various aldehyde reagents for their reactivity in transamination reaction (see Section 2.1.3).

Rather than demonstrating any ability to transaminate, 2PCA exclusively showed conversion to a pair of cyclic imidazolidinone
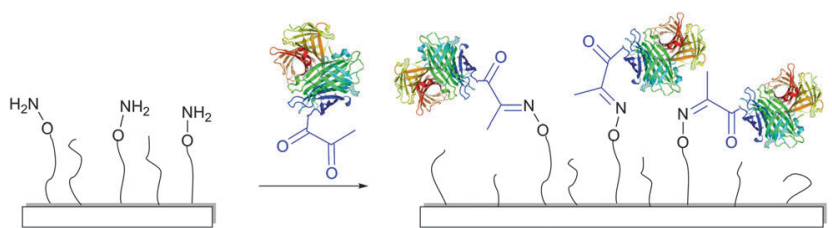

Fig. 71 Patterning of PLP-transaminated streptavidin (pdb: 4BX5) on aminooxy terminated polymer films. ${ }^{15}$ a)

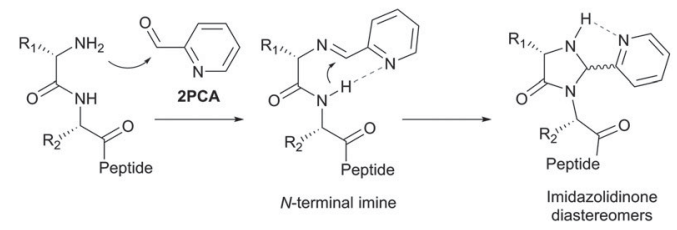

b)

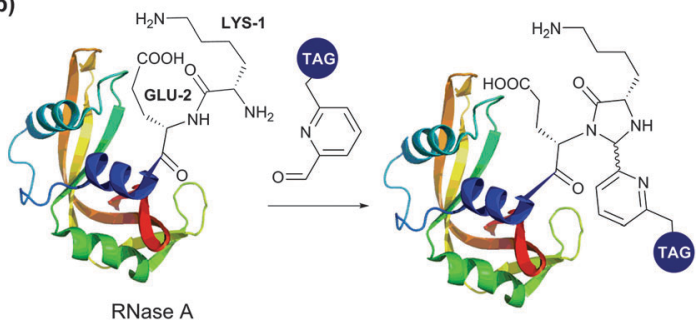

Fig. $72 \mathrm{~N}$-terminal protein modification using 2-pyridinecarboxyaldehydes (2PCT) reported by Francis et al. ${ }^{632}$ (a) Mechanism of the reaction of between $\mathrm{N}$-terminal amino group and 2PCA. (b) Modification of native RNase A (pdb: 7RSA).

diastereomers upon reaction with model peptides. The key step of the reaction mechanism is a nucleophilic attack of the adjacent amide nitrogen on the electrophilic carbon of the initially formed N-terminal imine (Fig. 72a). The presence of nitrogen heterocycles (namely pyridines) was found to be crucial for efficiency of this stereoelectronically disfavored condensation. It is noteworthy that Lys residues are unreactive in such a pathway, because of the lack of a neighbouring amide group suitable for cyclisation and higher $\mathrm{p} K_{\mathrm{a}}$ values compared to $\alpha$-amino groups. Furthermore, this methodology was also found to be compatible with the presence of free in-chain cysteines. This approach can therefore be considered as an orthogonal to other Lys-selective (see Section 1.1) and Cys-selective methodologies (see Section 1.3).

2PCA-mediated conjugation was found to be generally applicable for protein labelling (the authors demonstrated its application on a broad set of 12 different proteins including RNase A; Fig. 72b) except for $N$-acylated proteins (no imine formation) and peptides containing proline in position 2 (no cyclisation of the formed N-terminal imine). ${ }^{632}$ The resulting imidazolidinone-containing conjugates are generally moderately stable and decompose to starting protein by about $20-30 \%$ after 12 hours of incubation at $37{ }^{\circ} \mathrm{C}$ (in case of RNase A). This may limit the suitability of this methodology for several applications where stability of generated conjugates is crucial; however, the substrate variation could resolve the issue and such efforts are underway.

\subsection{Serine and threonine}

In 1960 Waller and Dixon have described the first procedure for selective N-terminal modification of peptides. It consisted of preparation of corticotrophin selectively acetylated on its terminal serine. ${ }^{633}$ Although being possible only under highly denaturing conditions of alkali exposure, the approach allowed for a spontaneous intramolecular $O \rightarrow N$ acyl transfer ${ }^{634}$ on the N-terminal Ser residues, while $O$-acetyl groups of in-chain Ser, Thr and Tyr residues were hydrolysed. The general idea of overcoming the 
entropy barrier of an otherwise slow intermolecular process by bringing two reacting partners together through a covalent linkage to initialize an intramolecular reaction has been later transformed into a large variety of selective modification reactions.

2.2.1 $O \rightarrow N$ shift of oxazolidines. 35 years after the early publication of Waller and Dixon, ${ }^{633}$ Liu and Tam ${ }^{635-637}$ have extended the applicability of $O \rightarrow N$ acyl transfer mediated methodologies by developing an approach to the chemical ligation of $\mathrm{N}$-terminal serine, threonine, and cysteine unprotected peptide segments with no need of using protecting groups. In order to make their methodology widely applicable, the authors proposed a general way to introduce an aldehyde function onto C-terminus by enzymatic coupling of a masked aldehyde, followed by chemical hydrolysis of the obtained intermediate. ${ }^{635}$ The key step of the process is the formation of the peptide bond through an intramolecular rearrangement between the two closely neighbouring carboxyl and secondary amino groups of formed of oxazolidine (in the case when Ser and Thr residues are involved) or thiazolidine (in the case of Cys residue, see Section 2.3.2) (Fig. 73). However, only in case when Cys-peptide was involved the reaction was found to be clean and no side products were observed. In contrast, when Thr- and Ser-peptides were used, it required refinement to ensure better yields. ${ }^{636}$

Although this methodology has demonstrated its high potential for the ligation of unprotected peptides, ${ }^{635,638,639}$ the generation of a "non-native" heterocyclic fragment at the site of ligating the two peptides made it extruded almost completely by other "native ligation" approaches; notably, by a similar mechanistically native chemical ligation (NCL) (see Section 2.3.1).

2.2.2 Periodate oxidation. A mild version of serine and threonine modification implies their prior conversion into glyoxyloyl derivatives via periodate oxidation first described by Fields and Dixon ${ }^{640}$ and later transformed into a general method for site-directed modification of proteins with N-terminal Ser or Thr by Geoghegan and Stroh. ${ }^{641}$

Based on the periodic acid mediated oxidation, ${ }^{642}$ the reaction occurs only when there exists the target site for the periodate to form a cyclic intermediate, that is to say, when the $\mathrm{N}$-terminal residue is represented by a serine or a threonine, or when hydroxylysine is present (rarely occurring in proteins). Possible side reactions include
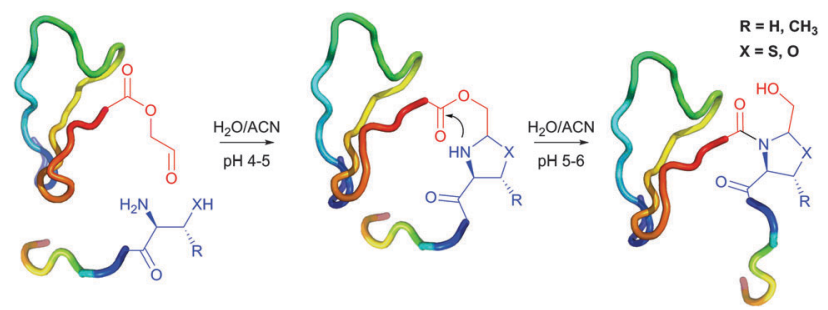

Fig. 73 General scheme of the ligation strategy proposed by Liu and Tam. ${ }^{635} \mathrm{~A}$ model 50 -residue peptide was obtained in good yield in ligation reaction between a 32-mer peptide VVSHFNDCPDSHTQFEFHGTCRFLVQEDKPAR containing C-terminal aldehyde function and a 17-mer peptide CHSGYVGARC(Ac-m)EHADLLA containing $\mathrm{N}$-terminal cysteine; in the case of Thr- and Ser-peptides, the reaction was moderately efficient. The peptides structures were simulated using the RaptorX web server. ${ }^{447}$

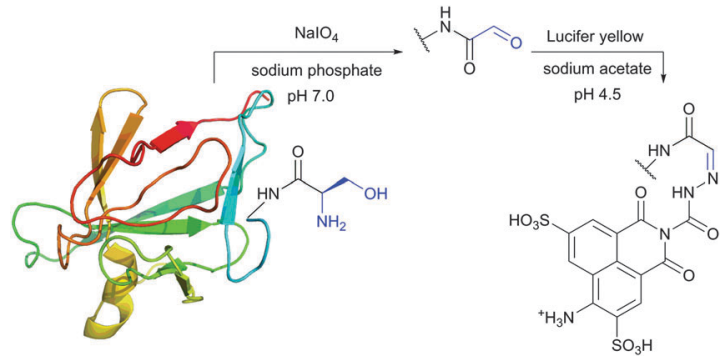

Fig. $74 \mathrm{~N}$-terminal serine labelling of recombinant murine interleukin-1 $\alpha$ (pdb: $2 \mathrm{KKI}$ ) with Lucifer yellow dye described by Geoghegan and Stroh. ${ }^{641}$ The method is also applicable if $\mathrm{N}$-terminal threonine is present.

oxidation of the side chains of Met, Trp, and His. However, the potential for side reactions can be diminished by using very low periodate-to-protein molar ratios, as demonstrated by Geoghegan and Stroh in their experiments on two model peptides, SIGSLAK and SYSMEHFRWG, and with recombinant murine interleukin- $1 \alpha$ (an $18 \mathrm{kDa}$ cytokine with N-terminal Ser, Fig. 74 ), ${ }^{641}$ or by conducting the oxidation at neutral $\mathrm{pH}^{643}$

As in the initial publication of Geoghegan and Stroh, ${ }^{641}$ obtained glyoxyloyl can serve as the locus for further chemical modification involving aldehyde-selective reactions (e.g. through the formation of stable oxime, hydrazone or previously described oxazolidine moieties). ${ }^{605,606}$ Robin and colleagues have demonstrated the possibility of using this two-step methodology for assembling two unprotected protein fragments: oxidised to glyoxyloyl-containing and hydrazide peptide derivatives. ${ }^{644}$ Rose and co-workers ${ }^{645}$ exploited the reactivity of generated glyoxyloyls towards $O$-alkyl hydroxylamine derivatives to synthesize a pentameric form of the cholera toxin subunit B.

Further investigation of periodate oxidation allowed its promoting for site-selective tagging, PEGylation, preparation of protein conjugates, protein capture and synthesis of large protein dendrimers. ${ }^{643,646-651}$ It is worth mentioning that periodate oxidation is incompatible with a number of protein classes. For instance, glycoproteins will undergo periodate-based cleavage of polysaccharide chains as a side reaction pathway. ${ }^{652}$

Lastly, glyoxyloyles can easily be transformed into corresponding amines via transamination reaction in the presence of copper(II) or nickel(II) salts. ${ }^{604,610,653}$ The reaction mechanism as well as need for both essential components of the system: the acceptor of the glyoxyloyl (usually aspartic acid or glycine) and the cation of a heavy metal are explained in Fig. 75. Despite being of moderate interest for bioconjugation by itself, this approach has initiated the development of a more general methodology for selective N-terminus modification - transaminative conjugation (Section 2.1.3). The reader is directed to a recent review by El Mahdi and Melnyk ${ }^{654}$ for a complete overview of the glyoxyloyl transformations in bioconjugation.

2.2.3 Phosphate-assisted ligation. Conceptually catching phosphate-assisted ligation at serine and threonine was recently reported by Payne and Thomas. ${ }^{655}$

The inherent reactivity of an N-terminal phosphorylated Ser or Thr residues was demonstrated to significantly facilitate the amide bond formation with a range of C-terminal peptide 


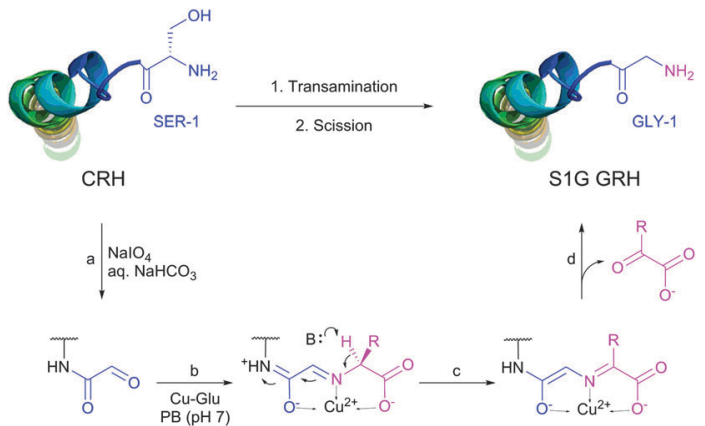

Fig. 75 Preparation of S1G mutant of corticotropin-releasing hormone $(\mathrm{GRH})$ by regioselective transformation of $\mathrm{N}$-terminal serine to glycine. ${ }^{604}$ Reaction steps: (a) selective serine oxidation by periodate; (b) formation of imine with amino group of glutamic acid precomplexated with $\mathrm{Cu}^{2+}$ (Cu-Glu); (c) base-assisted isomerisation of imine; (d) hydrolysis of imine, completed when aspartate and copper ions are removed.

thioesters. Although it is not yet clear what exact intermediate is formed during ligation, the authors have hypothesised that rapid acyl migration to the $\mathrm{N}$-terminal amine of a peptide occurs through the formation of unstable acyl phosphate (Fig. 76).

2.2.4 Indirect approaches. Ligation at Ser/Thr can also be achieved considering two distinct indirect approaches. Firstly,

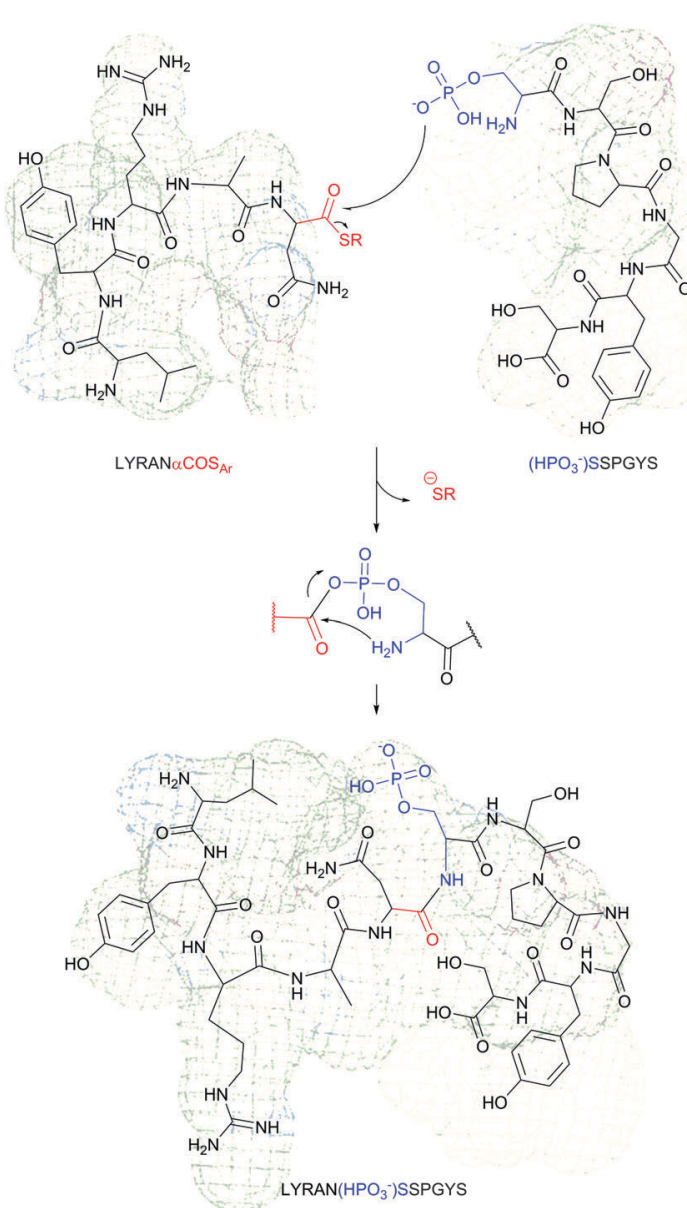

Fig. 76 Hypothesised mechanism of the phosphate-assisted ligation reported by Thomas et al. ${ }^{655}$
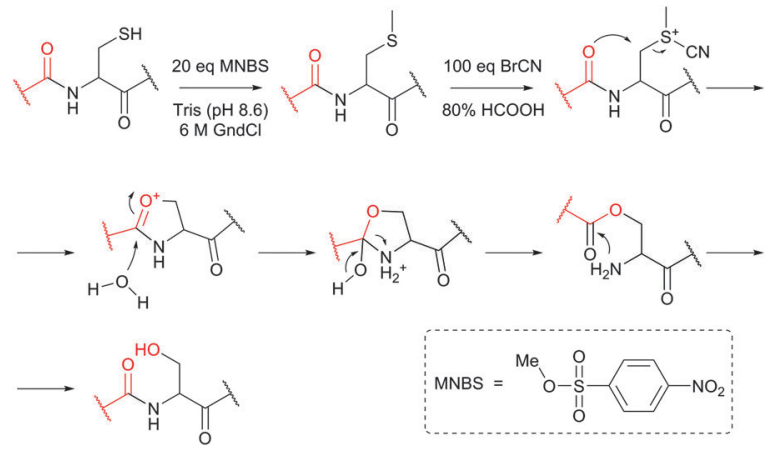

Fig. 77 Conversion of a Cys into a Ser residue by transforming the former into a corresponding methyl cysteine and following thereof by $\mathrm{CNBr}^{657}$ Methionine must be protected by transforming into corresponding sulfoxide form, because this is inactive for the CNBr reaction. ${ }^{658}$

as it was demonstrated by Danishefsky and co-workers, ${ }^{656}$ NCLdesulfurisation methodology can be used to access threonine at ligation sites (see Section 2.3.1.4). Secondly, as an alternative to the NCL-desulfurisation sequence, cysteine obtained during NCL can be chemically transformed into serine by methylation followed by activation of the obtained $S$-methylcysteine by cyanogen bromide (BrCN, Fig. 77). ${ }^{657}$

\subsection{Cysteine}

Generation of N-terminal Cys residue for native chemical ligation can be accomplished using solid-phase peptide synthesis, ${ }^{659}$ proteolytic processing, ${ }^{660}$ or by the spontaneous hydrolysis of intein fusion protein. ${ }^{661}$ Moreover, genetically directed, sitespecific incorporation of 1,2-aminothiols handle into proteins has been recently reported by Chin and associates. ${ }^{662}$

2.3.1 Native chemical ligation. The very principle of "chemical ligation" was coined by Kent in the early 1990s and consisted in an approach for covalent condensation of unprotected peptide segments by the means of "unique, mutually reactive functionalities designed to react only with each other and not with any of the functional groups found in peptides". ${ }^{663}$ That is, a general method that would enable the application of chemical tools to the world of the proteins. However, the original ligation chemistries exploited the reciprocal reactivity of chemical functions which are not present in native proteins and thus their prior introducing onto reacting partners is require and often associated with synthetic difficulties. Moreover, a non-native linkage is generated at the ligation site; therefore, many scientists remained sceptical about the validity of using such "analogous" proteins as tools for understanding the molecular basis of protein function.

In 1994, confronted with this criticism, Kent and co-workers ${ }^{64}$ introduced a versatile approach to the linkage of peptide fragments using a native peptide bond - native chemical ligation (NCL). Based on the original principles of the chemical ligation methodology ${ }^{663}$ and the ability of thioesters to undergo $S \rightarrow N$ acyl shift discovered by Wieland et al., ${ }^{665}$ NCL allowed to achieve chemoselective formation of the amide bond in the presence of unprotected nucleophilic amino acid side chains as alcohols 


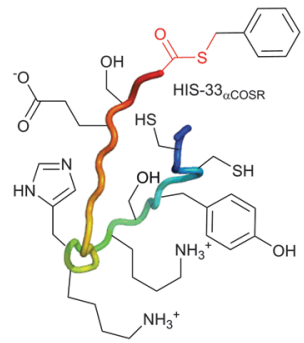

IL-8 (1-33 His- $\alpha$ COSR)
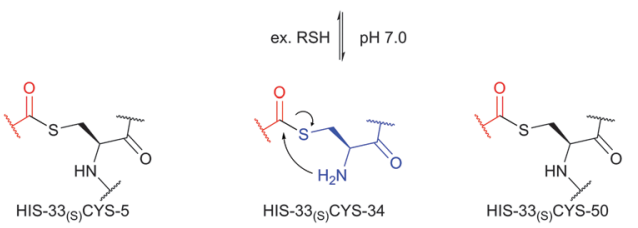

THIOESTER-LINKED INTERMEDIATES
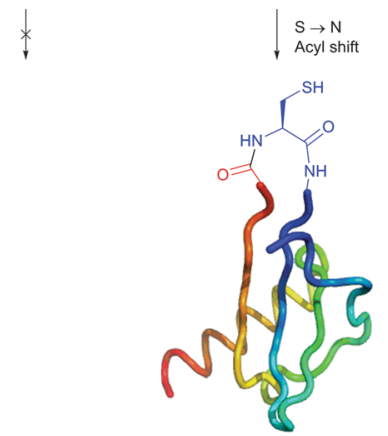

NATIVE POLYPEPTIDE (IL-8)

Fig. 78 Synthesis of the human interleukin 8 by native chemical ligation elaborated by Kent et al. ${ }^{664}$ The ligation reaction occurs between an unprotected peptide thioester fragment - IL-8(1-33 His- $\alpha$ COSR), and a second unprotected peptide possessing a N-terminal cysteine - IL-8(34-72). First step of thioester exchange results in different thioester-linked intermediates, among which only the peptide obtained from the corresponding $\mathrm{N}$-terminal cysteine can undergo following irreversible step of an $S-N$ acyl shift resulting in obtaining of a native amide bond at the linkage site.

(Ser/Thr), phenolates (Tyr), free amines (Lys), carboxylates (Glu/ Asp), or other thiolates (Cys) presented in the macromolecule.

By analogy with previously developed $O \rightarrow N$ acyl shift, the reversibility of the thioester-thiol exchange in the presence of an exogenous thiol additive coupled with the capture of the acyl segment by $S \rightarrow N$ acyl shift, being possible only in the case when the latter is brought in the close proximity to an amine in a $\mathrm{N}$-terminal cysteine thioester intermediate, result in an exquisite regioselectivity of this methodology. The product resulting from this $S \rightarrow N$ acyl shift represents a peptide, consisting of two fragments linked by a native peptide bond through a cysteine residue (Fig. 78).

Typically, the reaction performed in PS or PBS buffer $(\mathrm{pH}$ 7.0-8.5) at $37^{\circ} \mathrm{C}$ is complete in less than an hour and with high yields. ${ }^{666,667}$ Solubilizing agents such as guanidine hydrochloride or urea do not interfere with the ligation and are usually used to enhance the concentration of peptide segments, and thus increase reaction rate. It is important to prevent the thiolate of $\mathrm{N}$-terminal cysteine from the oxidation resulting in a disulfide-linked dimer, which is unreactive in the ligation.
A reductant (e.g. TCEP) or an excess of thiol corresponding to the thioester leaving group ( $4-5 \%, \mathrm{vol} / \mathrm{vol})$ is generally added to keep the Cys residues in reduced form. Moreover, the latter largely increases the overall rate of NCL by reversing the first step of transesterification for in-chain intermediate adducts deprived from the possibility to undergo $S \rightarrow N$ acyl shift and to generate a stable amide bond.

The first step in synthesizing a protein by NCL generally consists in defining the fragments to be used in the ligation reactions. Preferentially, naturally occurring AA-Cys motifs in the native sequence should be chosen as the ligation sites (AA stands for any amino acid). Val, Ile, Asp, Asn, Glu, Gln and Pro represent less favourable choices, because of lower ligation rates and possible side reactions, ${ }^{666}$ which, however, can be accelerated either by transformation of the corresponding thioesters into selenoesters, ${ }^{668}$ or by tuning the reaction $\mathrm{pH} .{ }^{669}$

Higher reaction rates were reported to be achievable while using good thiol-containing living groups, i.e. mildly acidic thiols such as thiophenol, 4-(carboxymethyl)thiophenol (MPAA), or 5-thio2-nitrobenzoic acid (TNB, the reduced form of Elman's reagent). These are generally generated in situ by thiol-thioester exchange from the relatively unreactive peptide- $\left({ }^{\alpha} \mathrm{COSCH}_{2} \mathrm{CH}_{2} \mathrm{CO}\right)$-Leu alkylthioester by adding an excess thereof $(1-5 \%$, vol $/ \mathrm{vol}){ }^{666}$

2.3.1.1 Sequential NCL. The complexity of proteins that can be obtained by NCL technique is limited by the maximum size of the accessible synthetic peptide segments. Two main approaches used today for the preparation thereof are: solid phase peptide synthesis pioneered by Merrifield (allowing the synthesis of proteins containing up to 50 residues), ${ }^{659}$ and recombinant DNA expression elaborated by Lobban et al. ${ }^{670}$ and Jackson et al. ${ }^{671}$ These allow for the preparation of peptide fragments containing up to 50, and up to 150 amino acid residues respectively.

Sequential native chemical ligation allows further extending of this limit by means of $\mathrm{N}$-terminal cysteine protected peptides. Three polypeptide fragments: a peptide ${ }_{1}-\mathrm{COSR}_{\mathrm{Ar}}$ (N-terminal fragment), a protected PG-Cys-peptide ${ }_{2}$ - $\operatorname{COSR}_{\mathrm{Ar}}$ (middle fragment), and a Cys-peptide $_{3}$ (C-terminal fragment), are thus assembled in a one-pot three step synthesis. Firstly, the middle fragment and the C-terminal fragment are ligated under the classical reaction conditions of NCL. Then protecting group is removed, uncovering N-terminal cysteine of the obtained polypeptide (central plus C-terminal fragment), which undergoes the second NCL with the N-terminal fragment to give the target protein (Fig. 79).

Since its introduction, sequential native chemical ligation has demonstrated its general applicability to the preparation of various complex assemblies. Consequently, several methodologies compatible with NCL for the protection of N-terminal Cys residues were elaborated. The most relevant among them are depicted in the Table 1.

The reader is referred to a recent review by Melnyk and collaborators $^{673}$ for a more complete overview of the sequential ligation strategies on proteins.

2.3.1.2 Kinetically controlled ligation. The simple observation that the reaction rate of NCL largely depends on the thioester 


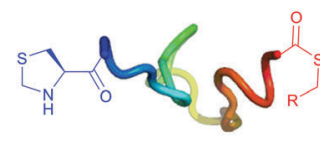

Thz-IGF(18-47) $\alpha \operatorname{COS}_{\text {Alk }}$
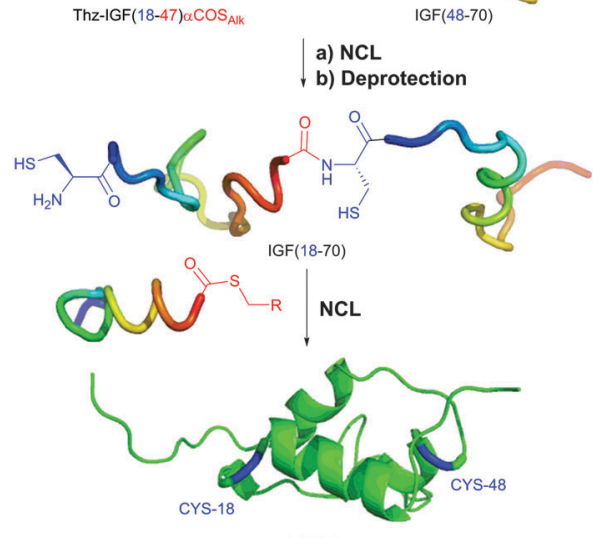

IGF-1

Fig. 79 Synthesis of insulin-like growth factor 1 (IGF-1) via sequential native chemical ligation described by Sohma et al. ${ }^{672}$ The reversible IGF(18-47) prevents its self-reaction with the $\alpha$-thioester moiety present in the same molecule. Thiazolidine protecting group can be easily removed by brief treatment with $\mathrm{NH}_{2} \mathrm{OMe} \cdot \mathrm{HCl}$ at $\mathrm{pH}$ 4. $\mathrm{NCL}$ reaction condition used: PB (pH 6.7), 6M GndCl, 10 mM MPPA, 20 mM TCEP.

nature was exploited by Bang et al. ${ }^{675}$ to introduce a convergent strategy for the synthesis of native peptides - kinetically controlled ligation (KCL).

The fact that the kinetics of NCL with alkylthioesters is significantly inferior of those with arylthioester makes it possible to control the intrinsic dual reactivity of a bifunctional Cys-peptide ${ }_{2}$ $\mathrm{COSR}_{\mathrm{Alk}}$ so that it would selectively react with a peptide ${ }_{1}-\mathrm{COSR}_{\mathrm{Ar}}$ and then undergo a classical NCL (i.e. in the presence of exogenous aryl thiol) with a third Cys-peptide ${ }_{3}$ to yield an assembled peptide -Cys-peptide $_{2}$-Cys-peptide -C $_{3}$ with no need to use protecting groups. Bang and collaborators have applied this methodology to assemble a 46-residue protein crambin from six peptide fragments (Fig. 80). protection of the $\alpha$-amino group of the central peptide fragment
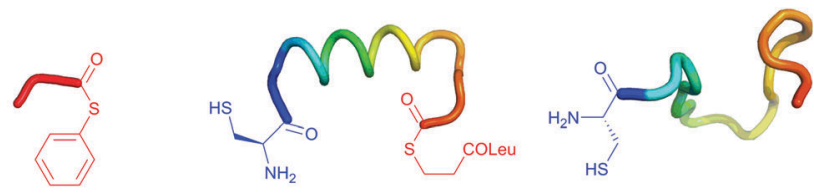

$\operatorname{TTC}(\alpha \operatorname{CosPh})$

CPSIVARSNFNACRLPGTPEAL $(\alpha$ COSAIk)

CATYTGCIIIPGATCPGDYAN

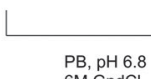

$\mathrm{PB}, \mathrm{pH} 6.8$
$6 \mathrm{M}$ GndCl

KCL

PB, $\mathrm{pH} 6.8$

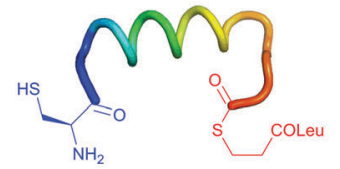

$6 \mathrm{M} \mathrm{GndCl} \quad \mathrm{NCL}$

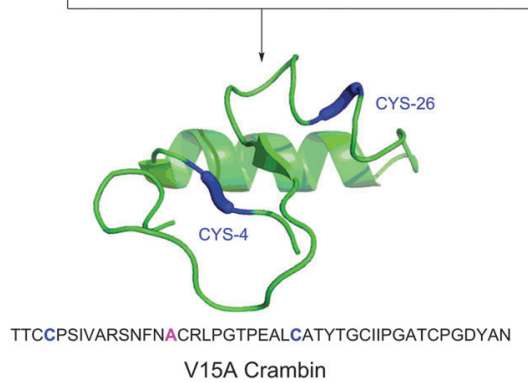

Fig. 80 Two final steps of the synthesis of V15A crambin (pdb: 3NIR) described by Bang and associated. ${ }^{675}$ The mutation was introduced for simplifying the prior KCL step of assembling the second peptide. Kinetically controlled ligation of $\mathrm{S}_{\mathrm{Ar}}$ thioester spontaneously occurs in aqueous media in the absence of exogenous thiophenol, while native chemical ligation of $\mathrm{S}_{\mathrm{Alk}}$ thioester must be accelerated by the addition of $1 \% \mathrm{PhSH}$.

Further advancing pioneering works done by Botti et al. ${ }^{686}$ on in situ acyl migration, KCL methodology has been recently extended from alkylthioesters to a full class of $O$-esters undergoing a spontaneous transformation to produce a thioester when exposed to a reducing agent through disulfide bond reduction followed by $O \rightarrow S$ acyl shift (Fig. 81). ${ }^{687}$

Through a thorough investigation Zheng et al. ${ }^{687}$ have defined that structures of the $O$-esters have an important effect on their reactivity. The authors have side-by-side benchmarked their methodology with previously described KCL by synthesizing the same V15A crambin (Fig. 80) by a one-pot one-step condensation of peptide segments and found its applicability to this system. Readily available by Fmoc solid-phase synthesis,

Table 1 Chemical functions generally utilised for the protection of $\mathrm{N}$-terminal cysteine during sequential native chemical ligation

\begin{tabular}{|c|c|c|c|}
\hline & Protecting group & Deprotection & Examples \\
\hline 1 & Thz & $\mathrm{NH}_{2} \mathrm{OMe} \mathrm{pH} 4$ & $\begin{array}{l}\text { IGF }{ }^{672}{ }^{6 I V-1 ~} \text { Tat }^{674}{ }^{67} \text { crambin }^{675,676} \\
\text { EPO, }{ }^{677} \text { PYP }^{678}\end{array}$ \\
\hline 2 & $\mathrm{Msc}^{679}$ & pH 12 & $\mathrm{SOD},{ }^{680} \mathrm{Abl-SH} 3^{681}$ \\
\hline $3^{a}$ & $\mathrm{Acm}^{682}$ & AgOAc, DTT & Crambin, ${ }^{683}$ DAGK $^{684}$ \\
\hline 4 & Mapoc $^{685}$ & $h \nu>300 \mathrm{~nm}$ & hBNP-32 685 \\
\hline
\end{tabular}

${ }^{a}$ Require a preparative-HPLC step before removal, gives lower overall yield compared with Thz. ${ }^{683}$ 

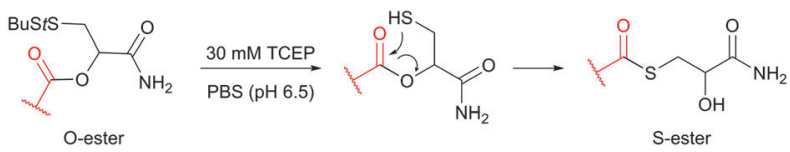

Fig. 81 Schematic representation of the $O \rightarrow S$ acyl shift of $O$-esters containing a disulfide bond described by Zheng et al. ${ }^{687}$

these $O$-ester scaffolds can expand the applicability of NCL to substrates with hardly accessible thioester peptide fragments.

\subsubsection{Access to C-terminal peptide thioesters}

Classical approaches. The preparation of C-terminal $\alpha$-thioesters involved in native chemical ligation is often associated with synthetic difficulties. Being especially reactive species, they either have to be introduced at the end of the synthetic pathway or be kept in a hidden form of thioester surrogates possessing higher stability.

Despite its recognised drawbacks due to hazardous acid treatment often leading to undesired side-reactions, the protocol of in situ neutralisation for Boc-based solid-phase peptide synthesis represents the most effective approach for the preparation of peptidyl thioesters. ${ }^{666,688-690}$ Alternatively, the Fmoc synthesis approach was investigated ${ }^{691-693}$ and found to be favoured when synthesizing phospho- and glycopeptides.

Expressed protein ligation (EPL). Introduced by Muir et al. in $1998,{ }^{694}$ EPL represents another approach for the preparation of $\alpha$-thioesters. It allows for obtaining the recombinant protein thioester by thiolysis of an intein fusion protein and thus enables a large pool of elaborated recombinant protein techniques for NCL. The reader is directed to several recent reviews of this area on chemical research for more details. ${ }^{695,696}$

$X \rightarrow S$ acyl-transfer. An elegant approach for keeping thioester in a relatively inert "hidden" form ready to be uncovered when required was first introduced by Danishefsky and colleagues in 2004 (Fig. 82). ${ }^{697}$ Several years later, the name "crypted thioesters" was coined by Otaka ${ }^{698}$ as a general term for such compounds. This approach is of especial interest, because it enables the assembly of peptide segments in $\mathrm{N}$-to-C direction, which is rather rare and often difficult to achieve. ${ }^{673}$ Indeed, all above-described methodologies rely on the assembling of polypeptide chain in $\mathrm{C}-\mathrm{to}-\mathrm{N}$ direction: for instance, the sequential native chemical ligation per se consists of iterated cycle of ligation-deprotection-ligation. .., i.e. adding new peptide fragments onto N-termini after deprotection thereof (see Section 2.3.1.1).

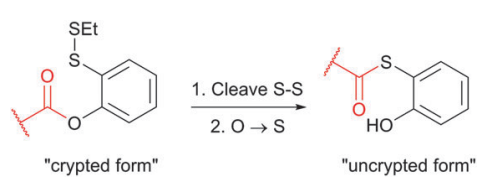

Fig. 82 Principle of "crypted thioesters" demonstrated by Danishefsky and co-workers. ${ }^{697}$ Intramolecular $\mathrm{O} \rightarrow \mathrm{S}$ migration of acyl residue resulting in generation of highly active thioester (its "uncrypted form") occurs only upon reductive cleavage of disulfide bond of reasonably stable "crypted form". a)
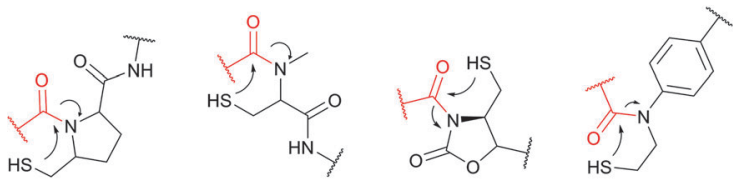

b)
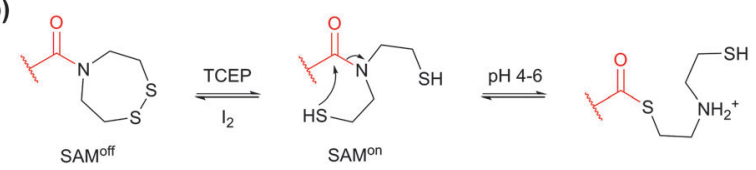

Fig. $83 \quad N \rightarrow S$ migration of acyl residue. The thioester-amide equilibrium is shifted towards the thioester form at low $\mathrm{pH}$ due to the protonation of the secondary amides. In the presence of 3-mercaptopropionic acid (MPA) all intermediates produce MPA-thioester. (a) From left to right: reduced form of Danishefsky's ester, ${ }^{697} 5$-mercaptomethyl prolines, ${ }^{701} \mathrm{~N}$-alkyl cysteamides, ${ }^{702}$ oxazolidinones, ${ }^{699}$ and $\mathrm{N}$-sulfanylethylamilides. ${ }^{703}$ (b) SAM ${ }^{\text {on/off }}$ approach by Melnyk and collaborators. ${ }^{711}$

Synthesis of thioesters in situ from stable amides via $N \rightarrow S$ acyl-transfer was demonstrated by Ohta et al. ${ }^{699}$ who studied acylated oxazolidinones derived from $S$-protected cysteine. These possess a distorted amide planarity provoking so called ground-state destabilisation ${ }^{700}$ and, as a consequence, favour the acyl migration to a deprotected thiolate. Nakahara and collaborators have studied and elaborated two classes of secondary amides amenable to the $N \rightarrow S$ acyl shift at low pH values: 5-mercaptomethyl prolines ${ }^{701}$ and $N$-alkyl cysteamides (Fig. 83a). ${ }^{702}$ Oxazolidinones $^{699}$ and $N$-sulfanylethylamilides (SEAlides) $^{703}$ described by Otaka and collaborators were found to possess similar aptitude towards $N \rightarrow S$ acyl-transfer shift at low $\mathrm{pH}$ (for a complete overview of $N \rightarrow S$ acyl-transfer systems described before 2010 see review by Kang and Macmillan). ${ }^{704}$ Erlich et $a .^{705}$ have recently applied $N$-alkyl cysteamide-based approach for the synthesis of 76-residue ubiquitin thioester, while Otaka and collaborators have demonstrated high potential of SEAlides by conducting the chemical synthesis of 162-redue active glycosylated GM2-activator protein. ${ }^{706}$

Almost simultaneously have two research groups reported a general approach, based on the application of bis(2-sulfanylethyl)amides (SAM) as precursors for NCL. ${ }^{707-710}$ An interesting extension of the methodology enabling the possibility of triggering the reactivity of SAM - so called SEA ${ }^{\text {on/off }}$ system - has been further elaborated by Melnyk and collaborators. ${ }^{711}$ The transition between reactive (SEA ${ }^{\text {on }}$ ) and unreactive (SEA ${ }^{\text {off }}$ ) states is simply triggered by mild oxidation/reduction procedures (Fig. 83b). $\mathrm{SEA}^{\text {off }}$ can be easily switched on via TCEP reduction, while the reverse switching off is achieved by mild oxidation with iodine. After few seconds, the excess of iodine is decomposed by the addition of dithiothreitol (DTT). Other amino acid residues susceptible to oxidation such as methionine or tryptophan are not affected because of this very short exposure to oxidant. However, cysteine residues must be protected with tertbutylsulfenyl groups to remain unaffected. At low $\mathrm{pH}$ values these are not reducible by DTT, allowing thus reliable protection of cysteines during the cycles of oxidative-reductive SAM triggering. 

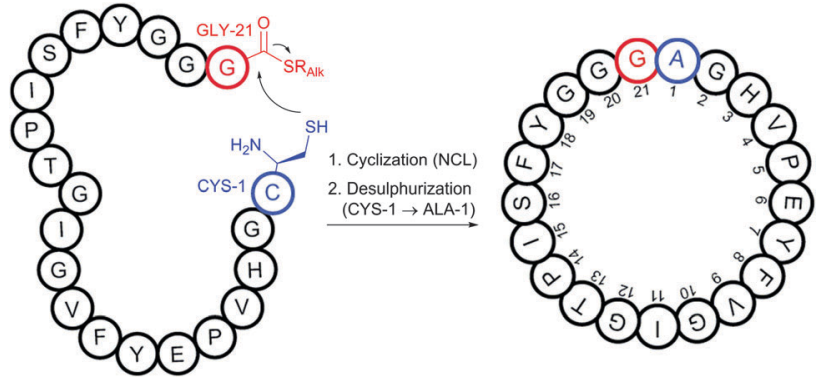

Fig. 84 Synthesis of 21-residue cyclic antibiotic peptide Microcin J25 via NCL-desulfurisation strategy resulting in alanyl-linked polypeptide chain developed by Yan and Dawson. ${ }^{712}$ Reaction conditions: cyclisation - Tris- $\mathrm{HCl}$, $6 \mathrm{M}$ GndCl; desulfurisation - NaOAc, pH 4.5, $6 \mathrm{M} \mathrm{GndCl,} \mathrm{H}_{2}\left(\mathrm{Pd}_{/} / \mathrm{Al}_{2} \mathrm{O}_{3}\right)$.

To demonstrate the potential of this newly established and optimised $\mathrm{SAM}^{\text {on/off }}$ system, Melnyk and collaborators have synthesised 85-residue domain of the hepotocyte growth factor (HGF 125-209). ${ }^{711}$

2.3.1.4 Indirect ligation on other amino acid using NCL. In 2001, Yan and Dawson ${ }^{712}$ have introduced an approach that, for the first time, allowed virtually conducting NCL at alanine. While the ligation still occurred at N-terminal cysteine, its subsequent desulfurisation with freshly prepared RANEY ${ }^{\mathbb{R}}$ nickel produced the native target sequence containing alanine residue at the ligation site (Fig. 84). This strategy has inspired the development of a large pool of various ligation junctions that includes phenylalanine, ${ }^{713,714}$ glycine, ${ }^{715}$ valine,${ }^{716,717}$ leucine, ${ }^{674,718}$ threonine, ${ }^{656}$ serine, ${ }^{657}$ proline, ${ }^{719-721}$ aspartate, ${ }^{722}$ glutamine, ${ }^{723}$ homocysteine, ${ }^{724,725}$ methionine (by subsequent $S$-methylation of the ligated homocysteinyl product by $p$-nitrobenzenesulfonate $)^{724}$ arginine ${ }^{726}$ and lysine ( $\alpha$ - or $\varepsilon$-selective) ligations. ${ }^{77,728}$

In order to extend native chemical ligation-desulfurisation approach to these amino acids, a SH-group should be attached to the carbon atom situated in the $\beta$-position to amino group (in some cases in the $\gamma$-position). The new building block containing a $\beta$-mercapto- $\alpha$-amino or $\gamma$-mercapto- $\alpha$-amino fragment is then introduced at the $\mathrm{N}$-terminal position during solid-phase peptide synthesis or by means of DNA-recombinant technologies. ${ }^{714}$ Following ligation is conducted under classic NCL conditions: at pH 7.5-8.0 in the presence of TCEP as reducing agent and 1\% of exogenous thiol additive. Finally, the desulfurisation of cysteine gives a nascent residue of interest (Table 2).

Besides aforementioned reduction on RANEY ${ }^{\mathbb{R}}$ nickel, various milder conditions such as nickel boride, ${ }^{712} \mathrm{Pd} / \mathrm{Al}_{2} \mathrm{O}_{3},{ }^{729}$ or metal-free conditions ${ }^{730,731}$ were developed to achieve efficient desulfurisation. More recently, in situ ligation-desulfurisation approach was also reported. $^{732,733}$

Desulfurisation-based methodology of the NCL expanding towards other amino acid junctions have contributed in many ways to prepare proteins and posttranslationally modified analogues for biochemical and structural analyses ever since its introduction. However, despite this broad utility, carrying out desulfurisation of the linkage site in the presence of other Cys residues in the protein sequence usually requires using of
Table 2 Desulfurisation-based NCL methodologies

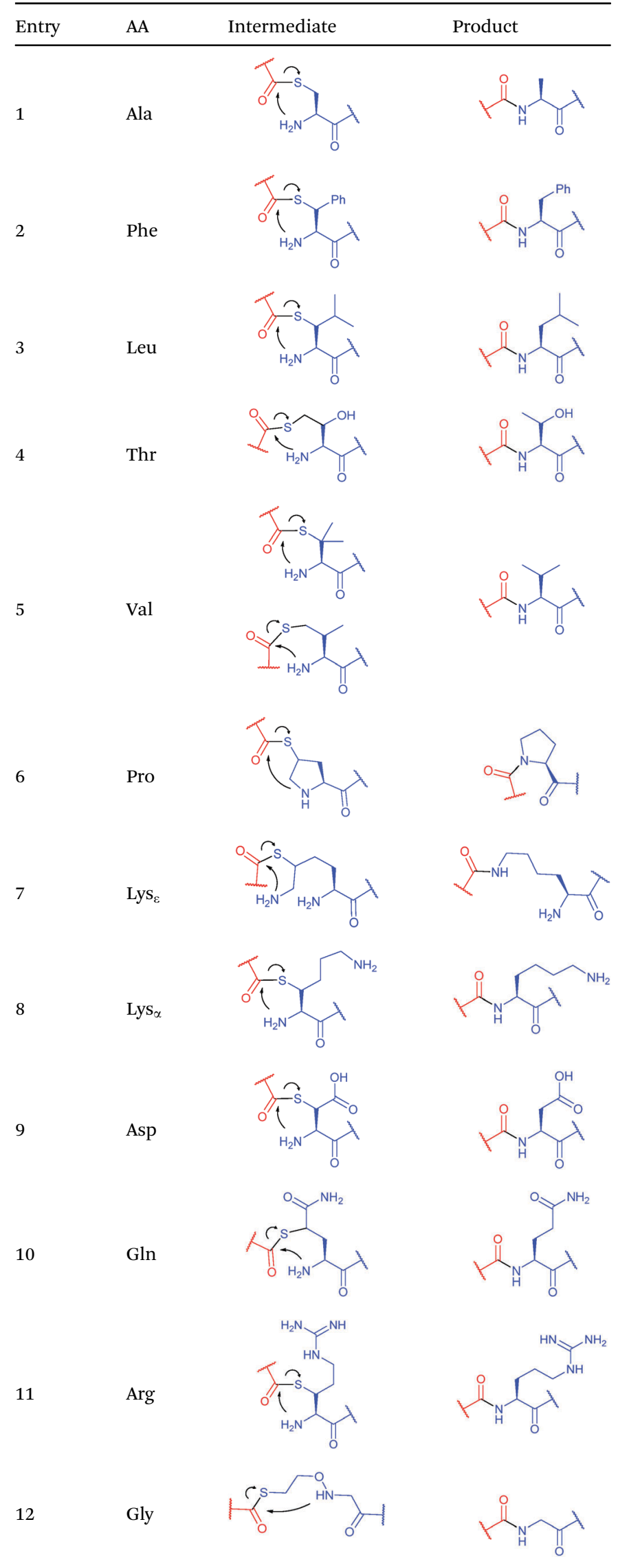


protecting groups. ${ }^{729,734}$ Not only does this necessity represent an undesirable step to protein synthesis, but it also implies some limitations on the applicability of the approach, mainly due to the solubility issues.

Developed by Dawson and collaborators ${ }^{735}$ the protocol for selective reduction of selenocysteines $(\mathrm{Sec}, \mathrm{U})$ in the presence of cysteine allowed overpassing the need for protection of thiolates and expanded the already established field of Sec-mediated native chemical ligations (see Section 2.6). ${ }^{736-739}$

The sensitivity of Sec peptides to reduction was noted in several works on selenocysteine ligations, ${ }^{737,738,740}$ During their preceding work on the synthesis of seleno-glutaredoxin 3 analogues (Se-Grx3), Dawson and associates ${ }^{741}$ have observed this incompatibility of Sec-containing proteins and peptides with TCEP-assisted native chemical ligation due to the generation of significant levels of a deselenised side product. In the publication to follow, the authors have successively applied the ligation-deselenisation strategy on a model peptide system. Accordingly, N-terminal Sec-peptide ${ }_{1}$ (UGLEFRSI-amide) prepared in the form of a diselenide dimer was ligated to the

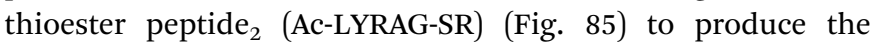
deselenised alanyl-peptide (Ac-LYRAGAGLEFRSI-amide) after the treatment with 50-fold excess TCEP at pH 5.5. Importantly, an excess of $200 \mathrm{mM}$ 4-mercaptophenylacetic acid (MPAA) was needed for the ligation step. MPAA is both served as a catalyst to activate the alkyl thioester and as mild reducing agent to generate a small pool of free selenol to facilitate the ligation reaction.

This selectivity of reduction with TCEP is hypothesised to be due to the weaker Se-C bond compared with the $\mathrm{S}-\mathrm{C}$ bond coupled with higher propensity of selenols to form radicals. ${ }^{742}$ It should be mention, however, that albeit upon heating, cysteine can be desulfurised in the absence of a radical initiator when treated with excess phosphine. ${ }^{743}$

Selenocysteine-mediated NCL deselenisation procedure have been recently exploited for selective ligation of selenolphenylalanine, ${ }^{74}$ and $\gamma$-selenolproline, ${ }^{720}$ easily transformable into peptides phenylalanine and proline respectively at the ligation site by treating Se-containing intermediates with TCEP or DTT. Analogously to $N \rightarrow S$ acyl transfer (Section 2.3.1.3), $N \rightarrow$ Se acyl shift was recently observed by Adams and Macmillan

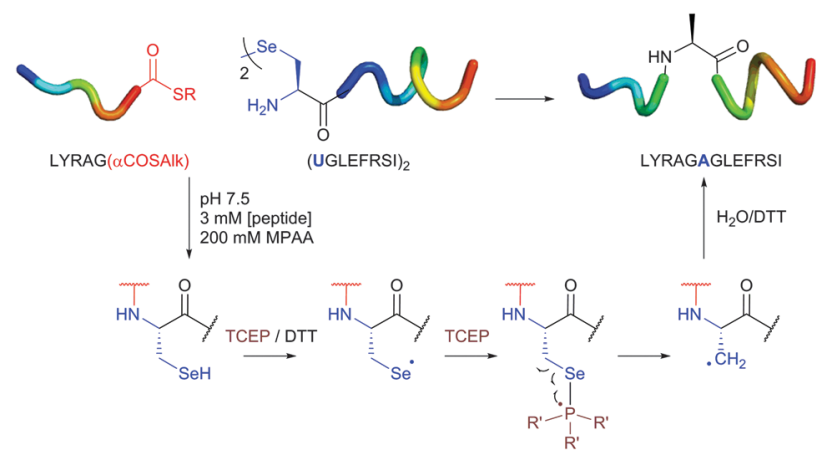

Fig. 85 Traceless ligation of peptides using selective deselenisation described by Dawson and collaborators. ${ }^{735}$ and allowed NCL to take place at lower temperatures and on shorter time scales. ${ }^{74}$ Corresponding selenoesters can be readily accessible by direct solid phase synthesis. ${ }^{746}$

NCL principles of $S \rightarrow N$ acyl transfer found their application in ligation assisted by proximity effect (see Section 5 ) ${ }^{747}$ allowing for conjugation of $\mathrm{N}$-terminal residues other that cysteine by auxiliary-mediated acyl transfer. Furthermore, the Cys side chain thiolate introduced during NCL can also provide a synthetic handle for further functionalisation using cysteineselective methodologies (see Section 1.2). It was recently demonstrated by Fang and co-workers that more accessible than thioesters simple phenyl esters could undergo native chemical ligation smoothly under the promotion of imidazole. ${ }^{748}$ Lastly, recently reviewed by Monbaliu and Katritzky ${ }^{749}$ Kemp's templatemediated thiol ligation, ${ }^{750-758}$ Tam's ligation by thiol/disulfide exchange, ${ }^{759-761}$ and other auxiliary-driven extensions of native chemical ligation, ${ }^{762,763}$ represent significant importance in the field of protein synthesis and can be considered as appropriate for bioconjugation.

2.3.2 Thiazolidine formation. Further expanding the applicability of the reaction of cysteine with formaldehyde described by Ratner and Clarke, ${ }^{764}$ Tam and collaborators have elaborated a method for the selective conjugation of $\mathrm{N}$-terminal cysteines with aldehydes, resulting in obtaining of stable thiazolidines. ${ }^{636,643,765-767}$ The reaction of 1,2-aminothiols readily occurs at slightly acidic $\mathrm{pH}$ of $4-5$, while the concurrent reaction of free amines with aldehydes results in obtaining Schiff bases reversibly under the same conditions.

The thiazolidine-mediated ligation was first applied by Tam and associates to the preparation of peptide dendrimers ${ }^{765,767}$ by attaching unprotected peptide dendrones containing Cys residues at their $\mathrm{N}$-termini to a branched core matrix with aldehyde functions. Botti et al. ${ }^{766}$ have transformed this approach to a general method for the preparation of cyclic peptides. Villain et al. have demonstrated that obtained thiazolidines (sometimes referred to as pseudo-prolines ${ }^{768}$ can be selectively cleaved by adding hydroxylamine derivatives, which react with aldehyde functions protected under the form of thiazolidine to form oximes. The authors applied this methodology for the covalent capture of proteins possessing N-terminal Cys or Thr residues (Fig. 86). ${ }^{769}$ Interestingly, under the same conditions $\mathrm{N}$-terminal Ser residues reacted only poorly.

Because of the recent advances in the semisynthesis of proteins and the encoding of 1,2-aminothiols into recombinant proteins, ${ }^{662,770,771}$ thiazolidine-mediated conjugation is now experiencing a reappraisal of its potential for bioconjugation. ${ }^{772,773}$ For instance, Casi et al. ${ }^{772}$ have exploited thiazolidine formation for the preparation of antibody-drug conjugates by site-specific incorporation of a potent drug, containing an aldehyde moiety, to engineered recombinant antibodies displaying a Cys residue at their N-terminus, or a 1,2-aminothiol at their C-terminus. ${ }^{772}$ Lastly, thiazolidines represent one of the most often used $\mathrm{N}$-terminal cysteine protecting groups for sequential NCL (see Table 1, Section 2.3.1.1).

2.3.3 2-Cyanobenzothiazoles (CBT). The reaction of 2-cyanobenzothiazole (CBT) with D-cysteine was first conducted by Field 


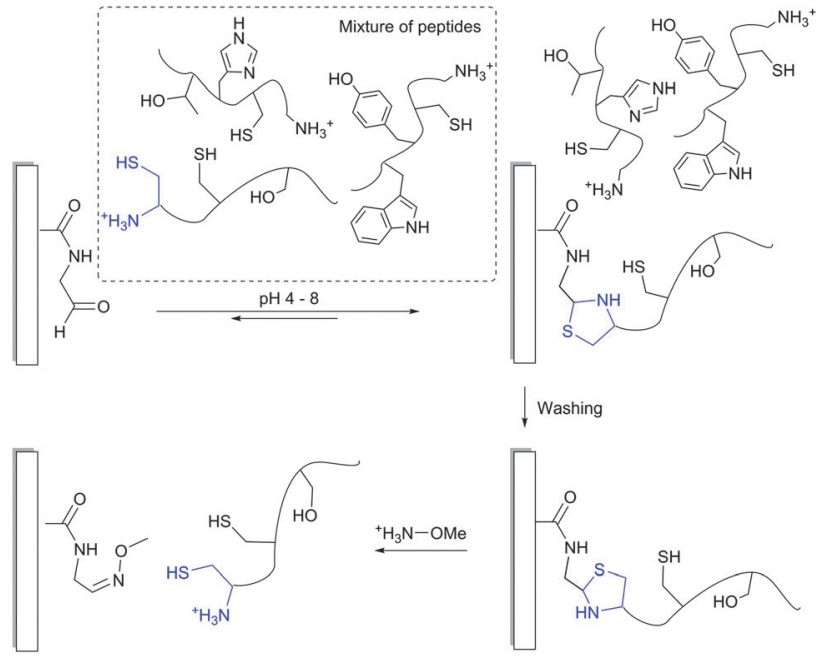

Fig. 86 General scheme of covalent capture purification of N-terminal cysteine containing proteins developed by Villain et al. ${ }^{769}$

and collaborators ${ }^{774}$ for the preparation of synthetic luciferin: a compound found in various living organisms and responsible for emitting light after being oxidised by a specific enzyme luciferase. Ever since its isolation $(9 \mathrm{mg}$ from 15000 firefly lanterns) ${ }^{775}$ luciferin enzymatic oxidation has been studied for the last 50 years. ${ }^{776}$

The regeneration pathway of luciferin in firefly was found to be consisted of the condensation of CBT with D-cysteine (Fig. 87). ${ }^{774}$ The reaction mechanism underlying this addition include first attack of cyano group of CBT by cysteine thiolate. This results in the formation of the electrophilic imidothiolate, subjected to the second attack by cysteine amino group to form thiazole structure after the yielding of ammonia gas.

Inspired by these early works, Rao and co-workers ${ }^{777}$ have further investigated the reaction of cyano-substituted aromatic compounds with amino-thiol substrates. They have demonstrated that benzotriazole motif plays an important role for the activation of the cysteine addition to a nitrile group. For instance, under optimal reaction conditions (PBS, $\mathrm{pH}$ 7.0-7.5) its replacement by other aromatic fragment such as picolinonitrile or benzonitrile largely decreases the reaction yield. All naturally occurring amino acids are unreactive towards CBT, except for cysteine owing the highest second-order rate constant among six other tested aminothiol substrates $\left(9.19 \mathrm{M}^{-1} \mathrm{~s}^{-1}\right.$, which is significantly higher than these of the majority of biocompatible click reactions). ${ }^{778}$ Finally, the efficiency and specificity of CBT-based labelling of terminal

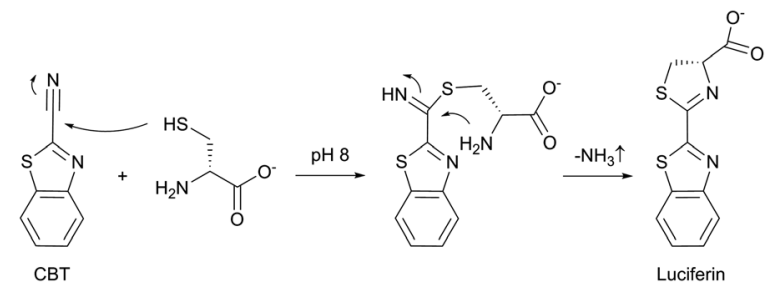

Fig. 87 Synthesis of luciferin by the reaction between cyanobenzothiazole (CBT) and D-cysteine. ${ }^{774}$

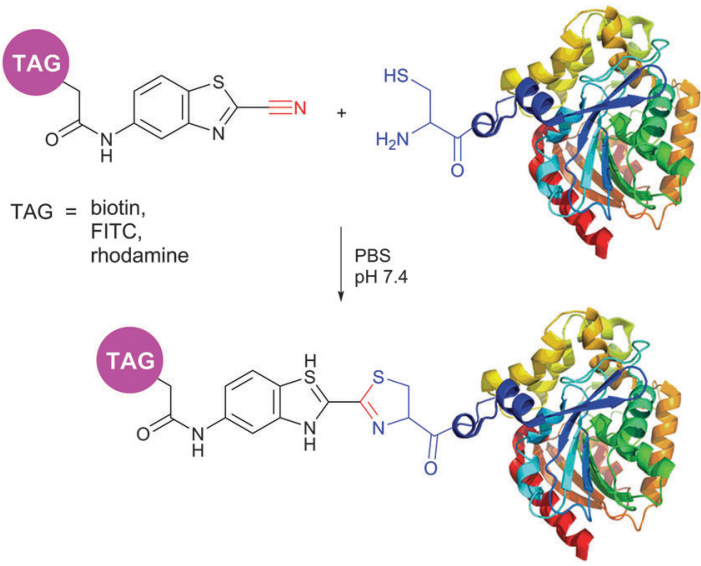

Fig. 88 Labelling of Renilla luciferase (pdb: 2PSD) by CBT-based probes. ${ }^{777} \mathrm{~N}$-terminal cysteine was generated by protease processing of the fusion protein.

cysteine residues was demonstrated on proteins in vitro (Fig. 88) as well as on cell surfaces.

In the publications to follow, Rao and colleagues have extended the applicability of CBT towards biocompatible condensations to create polymer assemblies in vitro and in living cells under the control of either $\mathrm{pH}$, disulfide reduction or enzymatic cleavage. ${ }^{779,780}$ Yuan et al. have taken advantage of this approach and developed a method for the determination of glutathione (GSH) concentration in vitro and in HepG2 human liver cancer cells. ${ }^{781}$ Jeon et al. ${ }^{782}$ have elaborated a CBT-based ${ }^{18} \mathrm{~F}$-probe radiolabelling of $\mathrm{N}$-terminal cysteine-bearing peptides and proteins. Two labelled substrates: a dimeric RGD-peptide $\left[{ }^{18} \mathrm{~F}\right] \mathrm{CBTRGD}_{2}$, and Renilla lucifierase bearing a cysteine at $\mathrm{N}$-terminus, have been synthesised with excellent radiochemical yields and shown good in vivo molecular PET imaging efficiency. Proceeding efficiently at physiological conditions, CBT-mediated $\mathrm{N}$-terminal Cys conjugation represents a useful alternative to existing approaches for protein labelling. ${ }^{783}$

\subsection{Tryptophan}

2.4.1 Sulfenylation-coupling. Encouraged by early reports from Scoffone and colleagues, who examined the site-selective modification of the nucleophilic 2-position of the tryptophan indole ring through electrophilic sulfenylation with various sulfenyl chlorides, ${ }^{784,785}$ Payne and collaborators have recently brought back into life a classical reagent for Trp selective modification - 2,4-dinitrophenylsulfenyl chloride (DNPS-Cl). ${ }^{786}$ The authors have demonstrated that, in acidic conditions, all nucleophilic amino acid side-chains except tryptophane (gives moderate yields of about $50 \%$ after 16 hours) either remained unmodified, as in the case of serine, threonine and the $\varepsilon$-amino groups of lysine, or were reversibly modified in the case of cysteine, which forms an easily reducible asymmetric disulfide. Further thiolytic cleavage of the resulting 2-Trp thioether derivatives with an external thiol nucleophile affords the corresponding 2-thiol Trp derivatives (2SH-Trp, Fig. 89a) in good yields. Interestingly, being placed on the N-terminus of the peptides, 2SH-Trp scaffolds were found to enhance the kinetics 
a)
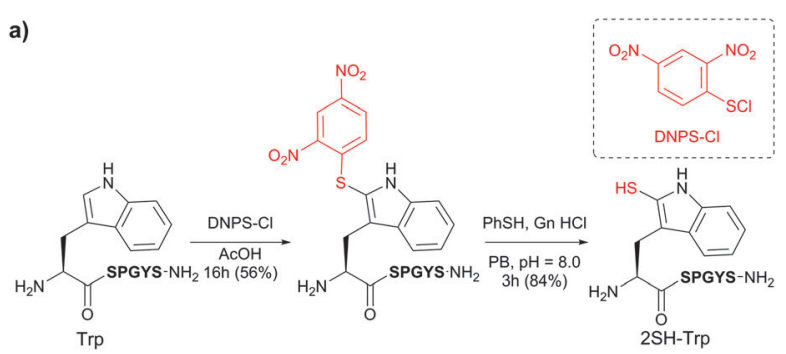

b)
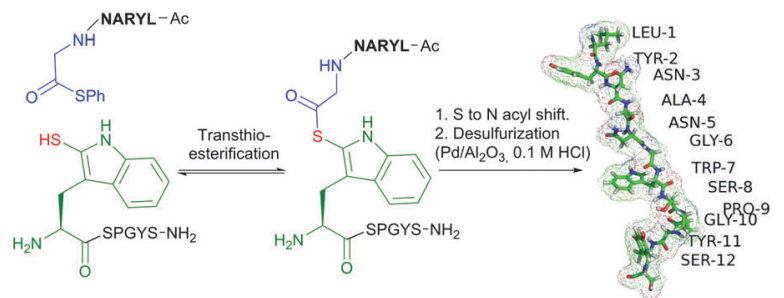

Fig. 89 Ligation-desulfurisation at tryptophan, reported by Payne and collaborators. $^{786}$ (a) Electrophilic sulfenylation of Trp in acidic conditions with DNPS- $\mathrm{Cl}$ and the obtention of the corresponding $2 \mathrm{SH}$-Trp derivative. (b) Auxiliary-assisted native ligation of $\mathrm{N}$-terminal $2 \mathrm{SH}$-Trp peptide $(2 \mathrm{SH}$ WSPGYS- $\mathrm{NH}_{2}$ ) with a thioester of the peptide Ac-LYRANG-SPh resulting in 12-mer peptide Ac-LYRANGWSPGYS after the desulfurisation step.

of the native ligation with peptide thioesters, and could thus serve as $\mathrm{N} \alpha$ acyl transfer auxiliaries (see Section 2.3.1). ${ }^{787}$

The proposed mechanism for this approach is mechanistically similar to NCL. It was hypothesised that the reaction would proceed via an initial step of the peptide thioester transthioesterification with an indole 2-thiol functionality followed by an $S$ - to $N$-acyl shift through a 7-membered ring transition state to generate a native amide bond. The last step of 2-thiol Trp desulfurisation results in obtention of a ligated product with only naturally occurring amino acid residues (Fig. 89b). Although this methodology represents a clever chemoselective approach for the ligation of completely unprotected peptide fragments through Trp moiety, the harshness of the reaction conditions of sulfenylation and desulfurisation limit it only to peptide substrated and don't allow its application on complex biomolecules.

2.4.2 Pictet-Spengler reaction. Another approach for the ligation of unprotected peptides was proposed by Li et al. ${ }^{788}$ and exploited the Pictet-Spengler reaction: an acid-catalysed intramolecular condensation between an iminium ion and an aromatic $C$-nucleophile described in $1911 .^{789}$ This non-natural ligation (peptides are linked by non-natural bonds) involves reaction of two peptide partners in acetic acid: one containing a Trp residue at its $\mathrm{N}$-term and another with a C-terminal aldehyde function. The latter should generally be introduced by means of solid-phase peptide synthesis on acetal resin (described by the same authors).

2.4.3 $N$-Acyl tryptophan isopeptides. Lastly, an interesting example of native $\mathrm{N}$-terminal Trp ligation, mechanistically very similar to NCL (see Section 2.3.1), was recently reported by Popov et al. ${ }^{790}$ The key intermediates of this methodology $\mathrm{N}$-acyl tryptophan isopeptides - undergo selective acyl transfer to yield natural peptides. These are, however, not accessible

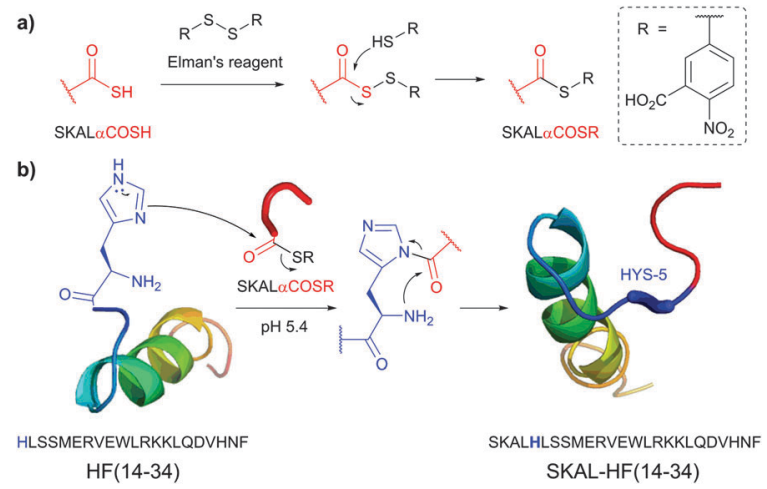

Fig. $90 \mathrm{~N}$-terminal histidine labelling described by Zhang and Tam. ${ }^{791}$ (a) Mechanism of thiocarboxylic acid activation by Ellman's reagent. (b) Labelling of bovine parathyroid hormone fragment (14-34, pdb: 1ZWC) with activated tetrapeptide thioacid.

directly by methods reported so far, which substantially restricts the applicability of the methodology.

\subsection{Histidine}

Despite being known for its particular importance for the acyl transfer in many enzymatic processes, ${ }^{665}$ histidine has been rarely used for bioconjugation. The only study describing the ligation of $\mathrm{N}$-terminal His peptides with activated thioester was reported by Zhang and Tam. ${ }^{791}$

Ellman's regent ${ }^{348}$ was used to activate the C-terminal peptide thiocarboxylic acid by forming acyldisulfide derivative, which is then nucleophilically attacked by $\mathrm{N}$-terminal histidine. Captured by the imidazole of the N-terminal histidine, the obtained $N_{\text {im }}$-acyl intermediate is hypothesised to undergo $N_{\mathrm{im}} \rightarrow N_{\alpha}$ shift to form histidine at the ligation site (Fig. 90). However, the

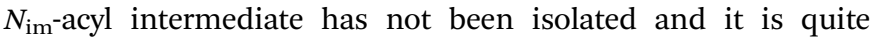
possible that regioselectivity is obtained simply because of anchimeric assistance of the proximal imidazole moiety at the ligation site.

Interestingly, no sign of coupling reaction has occurred when a corresponding non-activated C-terminal thiocarboxylic acid is participating in the reaction instead of the acyldisulfide. The reaction $\mathrm{pH}$ plays an important role on the effectiveness of the reaction. Only when maintained at slightly acidic values (pH 5-6) and in the absence of the thiol nucleophiles, would the imidazolyl moiety of histidine be the sole nucleophile present in the polypeptide. This methodology has been applied to generate histidine-containing peptides with yields up to $75 \%$.

\subsection{Selenocysteine}

Selenium and sulphur belong to the same main group of elements; therefore, 21st proteinogenic amino selenocysteine and Cys residue exhibit rather similar properties in terms of reactivity for bioconjugation. ${ }^{792-794}$ For instance, both Hilvert's ${ }^{738}$ and Raines's group ${ }^{736}$ demonstrated that C-terminal peptide thioesters react smoothly with peptide fragments containing a $\mathrm{N}$-terminal selenocysteine in exactly the same manner as with corresponding cysteine analogues. Presumably proceeding through the same mechanism as NCL (Section 2.3.1), the first 
step of the ligation process consists in the nucleophilic attack on the thioester by selenolate to give a selenoester intermediate that subsequently rearranges to give a native chemical bond.

Sec ligation can be chemoselective when conducted at slightly acidic $\mathrm{pH}$. Low intrinsic $\mathrm{p} K_{\mathrm{a}}$ values of selenocysteine $(5.2)^{795}$ and consequently its higher dissociation level at low $\mathrm{pH}$, endows this amino acid with unique biochemical properties, allowing regiospecific covalent conjugation with electrophilic compounds in the presence of the side chains of all other natural amino acids including the thiol group of Cys $\left(\mathrm{p} K_{\mathrm{a}}\right.$ 8.3). For instance, the reaction rate with selenocysteine was found to be 1000 fold faster than with cysteine at $\mathrm{pH} 5.0 .^{736}$ Moreover, the lower $\mathrm{pH}$ generally suppresses $\beta$-elimination of the selenol group from selenocysteine resulting in the obtaining of unreactive dehydroalanine. ${ }^{392}$

Initially, considerable efforts were made to show the applicability of selenocysteine NCL for the preparation of selenium-containing derivatives of enzymes and benchmarking activities thereof. Hilvert and associates synthesised a $\mathrm{C} 38 \mathrm{U}$ analogue of bovine pancreatic trypsin inhibitor (BPTI). Amusingly, the wild-type BPTI and its artificial analogue folded into alike conformations and demonstrated similar inhibiting affinity of trypsin and chymotrypsin. ${ }^{738}$ Raines et al. selected 124-residue ribonuclease A (RNase A) as a model protein for the investigations. ${ }^{736}$ DNA recombinant technology was utilised to prepare a C-terminal thioester fragment corresponding to residues 1-109, while standard solid phase peptide synthesis methodology was used to obtain a N-terminal Cys and Sec peptides corresponding to residues 110-124 (Fig. 91). Just as in the case of BPTI, the semisynthetic wild-type RNase A and C110U RNase A presented equivalent ribonucleolytic activities. Further advances in the field of Sec-NCL have resulted in synthesis and investigations of other different proteins such as seleno-glutaredoxin, ${ }^{796}$ azurin, ${ }^{797,798}$ and thioredoxine reductase.

Ease of the post-ligation transformation of selenocysteine to alanine (by deselenisation), dehydroalanine (by $\beta$-elimination) or non-natural amino acids (by addition reaction to dehydroalanine, see Section 1.3.8) became a spur to a further propagation of Sec-mediated methodologies as very effective tools in rational design of peptides and proteins. Quaderer and Hilvert ${ }^{799}$ exploited such transmutations of selenocysteine to access a series 16-residue cyclic peptides (Fig. 92).

In this initial report, the deselenisation step was conducted rather harshly (RANEY ${ }^{\circledR} \mathrm{Ni}, \mathrm{H}_{2}$ ), implying that all Cys residues (if there were any) would have been reduced as well. Recently, however, Dawson and collaborators ${ }^{735}$ have demonstrated that

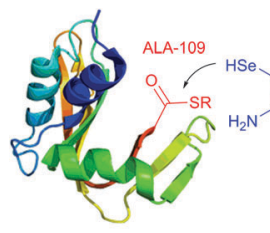

RNase A (1-109)
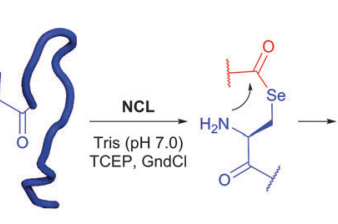

ig. 91 Selenocysteine native chemical ligation applied for the preparation of C110U mutant of RNase A (wild-type - pdb: 7RSA). ${ }^{736}$

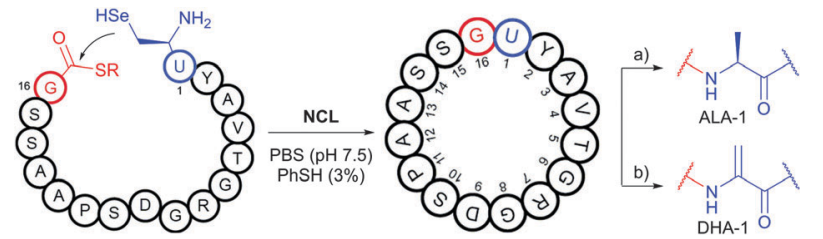

Fig. 92 Synthesis of cyclic 16-resiudes selenopeptides by native chemical ligation and following transformation thereof to (a) alanine (RANEY ${ }^{\circledR} \mathrm{Ni}$, $\mathrm{H}_{2}$, TCEP, 20\% AcOH); (b) dehydroalanines $\left(\mathrm{H}_{2} \mathrm{O}_{2}, 10 \% \mathrm{ACN}\right)$.

selenocysteine can be chemoselectively deselenated with TCEP in the presence of cysteines. This allowed overpassing the main limitation of the NCL-desulfurisation strategy (see Section 2.3.1.4), namely the inability to control regioselectivity of desulfurisations if several cysteines are present in the peptide or protein, and yielded in a pool of NCL-deselenisation strategies for mild incorporation of alanine, phenylalanine and proline into the ligation site by classic NCL approaches (see Table 2). Finally, selenocysteine peptides were found to efficiently undergo reverse NCL at acidic $\mathrm{pH}$ and thus to be of particular interest for the generation of thioesters by sequential $N \rightarrow$ Se acyl-transfer and substitution of the obtained selenoester by exogenous thiol (see Section 2.3.1.3). ${ }^{745}$

Because the incorporations of selenocysteine by the cell translational machinery are generally very laborious, ${ }^{800,801}$ selenopeptides are mainly obtained by SPPS. ${ }^{736,737,794}$

\subsection{Proline}

The oxidative coupling of $o$-aminophenols and $o$-catechols recently reported by Francis and collaborators ${ }^{802}$ represents an interesting approach for selective $\mathrm{N}$-terminal conjugation on proteins. The strategy consists in prior in situ oxidation of $o$-aminophenols and $o$-catechols to active coupling species using potassium ferricyanide (Fig. 93a) followed by their reaction with a protein (Fig. 93a). This approach was shown to work particularly well with proline (due to increased nucleophilicity thereof) and can therefore be considered as N-terminal Pro selective methodology. ${ }^{802}$ The key advantage of the approach compared to the majority of N-terminal selective methodologies is its fast second-order

a)
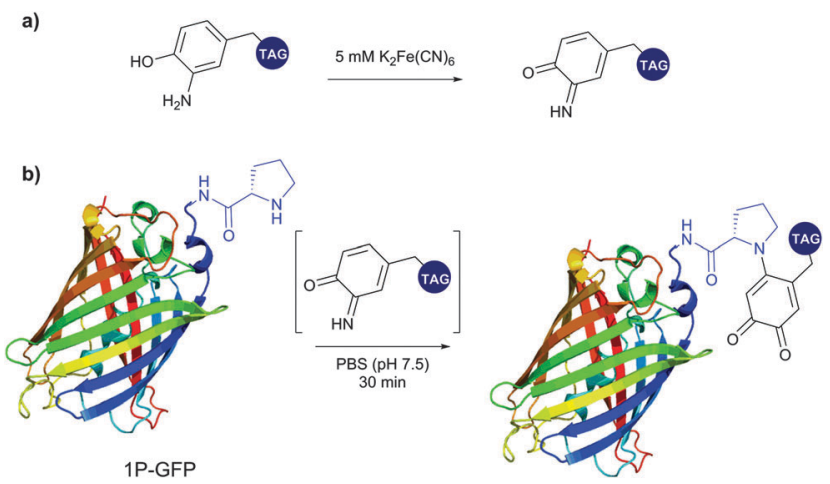

Fig. 93 Selective modification of 1P-GFP (Pro residue is introduced on $\mathrm{N}$-terminus) with o-aminophenol-PEG reagent (wild-type - pdb: 1GFL). ${ }^{802}$ (a) In situ oxidation of 0 -aminophenol derivatite. (b) Reaction of the active coupling species with $\mathrm{N}$-terminus of $1 \mathrm{P}$-GFP. 
kinetics in a single step transformation, which does not require prior oxidation of the N-terminus. Free cysteine residues, however, are also reactive during the oxidative coupling and must thus be protected, which represents the main drawback of the approach.

\section{C-terminal conjugation}

Chemical methods for C-termini conjugation are rather scarce. Definitely, previously mentioned approaches for the conjugation of side-chain carboxylates (see Section 1.5) can also be applied effectively to modify the terminal carboxylates. The selectivity, however, would be the main issue, for up to date there is no described method for somewhat selective activation of $\mathrm{N}$-terminal carboxylates by any activating reagent.

Because of the impetuous development of NCL (see Section 2.3.1), requiring C-terminal thiolates as reacting partners for $\mathrm{N}$-terminal Cys proteins, those became widely accessible, namely by means of SPPS. A perspective approach, exploiting these advances was proposed by Goody ${ }^{803}$ and collaborators, who developed a protocol for selective transformation of C-terminal thioesters to corresponding hydroxylamines, enabling thus the application of aldehyde- and ketone-selective methodologies on the C-terminus.

On the other hand, the unique position of protein C-termini has stimulated numerous efforts to target this location, which resulted in numerous enzymatic and intein-based approaches for C-terminal-selective protein modification. ${ }^{804-809}$ These methodologies are, however, not covered by this review devoted to chemical methods of bioconjugation.

\section{Sequence-selective approaches}

Several especially useful methodologies in bioconjugation exploit not a specific property of the residue, or its peculiar position in protein, but rather the synergetic effect of a batch of neighbouring amino acid residues. For instance, an example of such selectivity is the aforementioned selective modification of His-AA-Ser and His-AA-Thr peptide motifs (see Section 1.2) by usually promiscuous activated esters. In this case, the His imidazolyl side chain located in close proximity to Ser/Thr side chains increased the reactivity thereof towards electrophiles.

Metal-chelation methodologies are perhaps the most elaborated among sequence-selective approaches (Fig. 94). ${ }^{810}$ The oligohistidine sequence (usually $\mathrm{H}_{6}$ ), called His-tag, is known to interact robustly with transition-metal complexes, including a nitrilotriacetic acid (NTA) complex of $\mathrm{Ni}^{2+}$, therefore the sequence is widely used for protein purification by affinity chromatography. Similarly, oligoaspartates (most often $\left(\mathbf{D}_{4}\right)_{n}, n=1-3$ ) were developed for selective labelling with multinuclear $\mathrm{Zn}^{2+}$ complexes. ${ }^{811,812}$ The tetracysteine motif (CCPGCC) was reported to especially effectively chelate biarsenical dyes such as $4^{\prime}, 5^{\prime}$-bis(1,3,2-dithioarsolan-2-yl)fluorescein (FlAsH) ${ }^{5,813}$ while a similar structural tetraserine sequence (SSPGSS) was found to be selective for diborate-scaffolds.
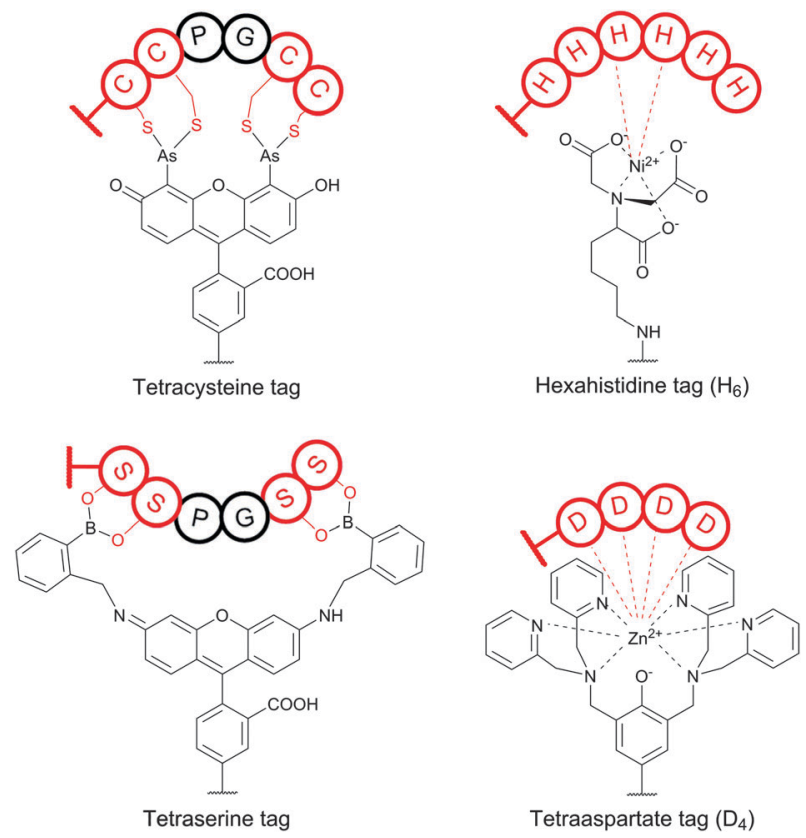

Fig. 94 Strategies for the selective conjugation of proteins based on metal-chelation: tetracysteine/biarsenical system, oligohistidine/nickelcomplex system, tetraserine-borate system, oligo-aspartate/zinc-complex system. $^{27}$

\section{Proximity-driven modifications}

All the above-mentioned methodologies are mainly residuespecific. That is to say, they exploit specific reactivity of the functional group of interest or of an assembly of residues. As a result, bioconjugations of highly nucleophilic Cys, Lys, and Tyr residues with electrophilic reagents are definitely prevalent among described methodologies.

The inherent reactivity is rare to be overcome, which largely limits of the scope of known methods for bioconjugation of the amino acids possessing low nucleophilicity. However, bringing the reaction partners into close proximity can accelerate a reaction thereof, which would not otherwise be possible because of the presence of other more reactive species. Routinely exploited by enzymes, this approach enables selectivity on the basis of the molecular shape rather than reactivity or the local environment (Fig. 95).

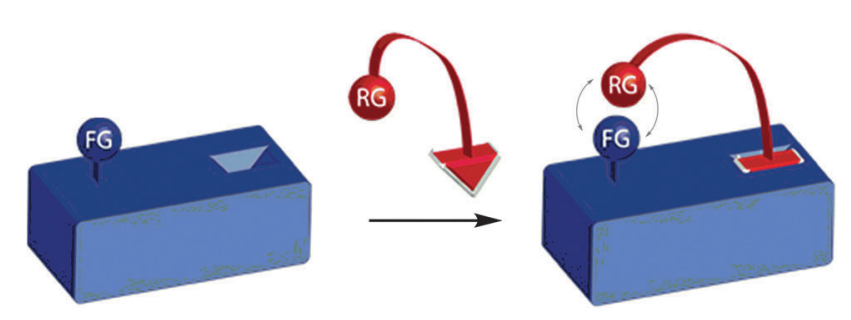

Fig. 95 Schematic representation of the proximity-driven approach for selective modification of macromolecules. Combining of promiscuous reactivity of the reactive group (RG, shown in red) with specific molecular recognition results in specific modification of the functional group of interest ( $F G$, shown in blue). 
Developed by Hamachi and collaborators in $2006,{ }^{462}$ the post-affinity-labelling approach can be used for the selective tethering a functional molecule at the proximity of the active site of enzymes. This method was then used on numerous substrates and enlarged the scope of selective conjugation especially on histidine and tyrosine (see Section 1.5.1). ${ }^{462,463,486,814}$ Despite these successes, a prevalent limitation of the applicability of the method due to the possible modification of the residues situated only in the vicinity of ligand-binding pocket of the target protein prevents this methodology from becoming a general approach for protein labelling.

In 2010, exploiting similar idea, Popp and Ball ${ }^{454}$ envisioned the combination of two previously described techniques: the coiled-coil based molecular recognition of complementary peptides ${ }^{455,815-817}$ and high catalytic activity of dirhodium complexes on carbene $\mathrm{C}-\mathrm{H}$ insertion, previously reported by Francis and collaborators ${ }^{452}$ for the selective modification of tryptophan (see Section 1.4.2). Two complementary peptides: one, containing a dirhodium catalytic centre (precomplexated through two glutamate side chains), and another, containing a side chain to be modified are thus involved in this methodology (Fig. 96a). Because of the compelled proximity of the side chain of interest and the active catalytic centre in the obtained supramolecular assembly, the reaction of rhodium-catalysed $\mathrm{C}-\mathrm{H}$ insertion is largely accelerated (more than $10^{3}$ times). ${ }^{818}$

As a result, the conjugation of amino acid residues with lower reactivities becomes possible. This allowed to expand the scope of originally tryptophan-selective dirhodium carbene methodology first to the other aromatic residues, phenylalanine and tyrosine, ${ }^{454}$ and then to over half of the naturally occurring amino acid residues (Fig. 96b). ${ }^{818}$ To date, dirhodium metallopeptide represent the only reported method for selective modification of Gln, Asn, and Phe side chains. The authors have also demonstrated the possibility to apply their methodology on chimeric proteins, containing fused coils, ${ }^{456}$ as well as on full-sised natural proteins possessing coiled-coils in their structures. ${ }^{818}$

Despite its important potential, the metallopeptides methodology is however not devoid of drawbacks. Because binding to dirhodium is nonselective and thus cannot be performed in the presence of other carboxylate-containing peptides, rhodiumpeptide complexes must be synthesised beforehand, which is often challenging mainly due to their poor solubility. ${ }^{819}$ Moreover, the method is restricted to proteins containing coiled coil fragments in their structures, which in case of the vast majority of targets would mean the need for resource- and timeconsuming expression of fused proteins.

Another approach, developed by Silverman and colleagues, exploits self-assembling of complementary DNA to bring into proximity two reacting fragments and allowed, although only on simple substrates, selective phosphorylation of tyrosine and serine, otherwise not feasible. ${ }^{538}$

Beyond coiled coils and DNA-based preorganisation, the principles of proximity-driven selectivity should be extended to other helix-binding protein domains and to biological molecular recognition generally. A significant broadening of the applicability of this elegant approach for protein modification,

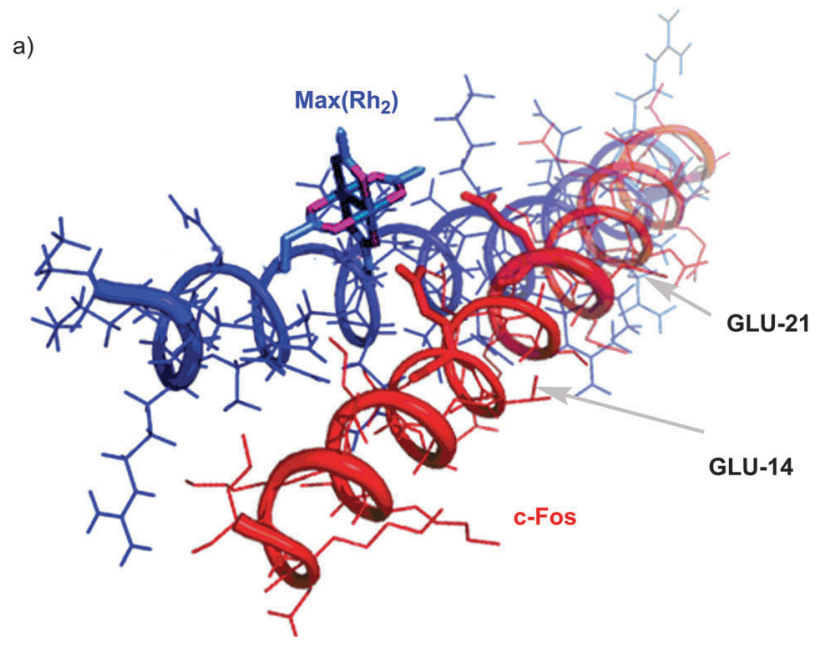

b)

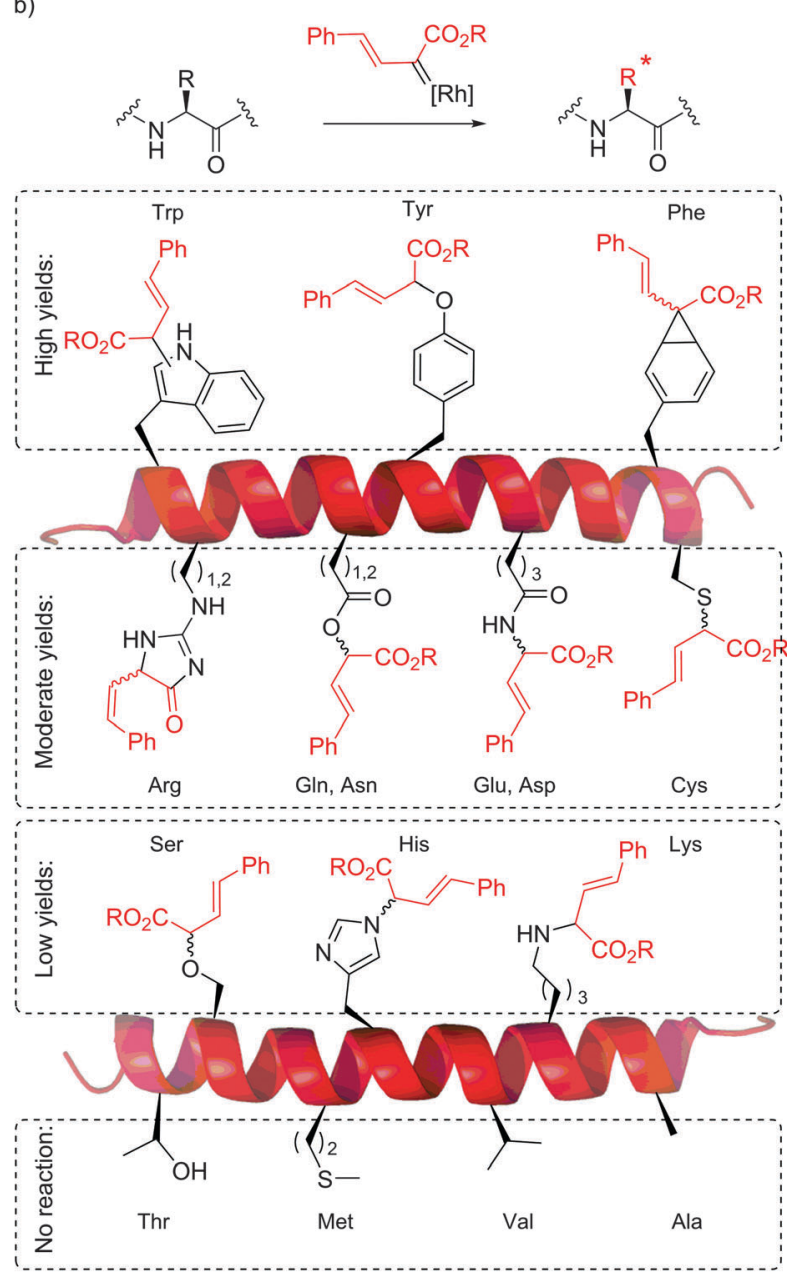

Fig. 96 (a) Modification of C-Fos (shown in red) catalysed by $\operatorname{Max}\left(\mathrm{Rh}_{2}\right)$ (shown in blue) metallopeptide described by Popp and Ball. ${ }^{818}$ Two possible coiled-coil alignments result in modification of GLU-14 and GLU-21 residues, located in close vicinity to the dirhodium catalytic center. (b) Proposed product bond connectivity.

biochemistry and biomaterials engineering is anticipated in the nearest future. 


\section{Conclusions}

The field of bioconjugation has expanded in the last 100 years and passed from a blind-eye modification of proteins one could found in nature to a well-established independent domain full of approaches allowing precise and reliable introduction of various tags into proteins' structure.

Many, if not most of these methods, however, often possess drawbacks limiting their general applicability. This fact has become of special consideration with a rise of novel exigent applications for bioconjugation, namely the preparation of new therapeutic conjugates, vaccines, and biomaterials. Also tremendous progress in the sensitivity of analytical methodologies as well as the need to work with smaller and smaller amounts of sample, often-unstandardized patient samples, highlighted the need for more efficient, selective and reliable bioconjugation methods.

Moreover, some parameters of the mode of conjugation, previously completely neglected, were recently revealed to be of paramount importance. For instance, the stability of the generated linkage and the distribution of products generated upon conjugation can be determining for the overall efficiency of the conjugate.

Overall, we believe that intensive ongoing research in the field of bioconjugation will result in more efficient and selective methodologies allowing specific conjugation of native proteins in complex biological media, and ultimately in living organisms.

\section{Acknowledgements}

We thank Dr S. Kolodych (Syndivia SAS), Dr J.-Y. Bonnefoy (Syndivia SAS), Dr A. Osypenko (UDS), and Dr M. Recher (UDS) for fruitful discussions that led to the redaction of this manuscript. We also acknowledge the financial support from SATT Conectus "BioReLiable" project, CNRS, University of Strasbourg, and International Center for Frontier Research in Chemistry (icFRC).

\section{References}

1 S. Zalipsky, Bioconjugate Chem., 1995, 6, 150-165.

2 J. M. Harris and R. B. Chess, Nat. Rev. Drug Discovery, 2003, 2, 214-221.

3 D. P. Baker, E. Y. Lin, K. Lin, M. Pellegrini, R. C. Petter, L. L. Chen, R. M. Arduini, M. Brickelmaier, D. Wen, D. M. Hess, L. Chen, D. Grant, A. Whitty, A. Gill, D. J. Lindner and R. B. Pepinsky, Bioconjugate Chem., 2006, 17, 179-188.

4 S. J. Bell, C. M. Fam, E. A. Chlipala, S. J. Carlson, J. I. Lee, M. S. Rosendahl, D. H. Doherty and G. N. Cox, Bioconjugate Chem., 2008, 19, 299-305.

5 B. A. Griffin, Science, 1998, 281, 269-272.

6 H. M. O'Hare and K. Johnsson, Curr. Opin. Struct. Biol., 2007, 17, 488-494.

7 X. Michalet, S. Weiss and M. Jäger, Macromolecules, 2006, 106, 1785-1813.

8 C. A. Royer, Chem. Rev., 2006, 106, 1769-1784.

9 C. R. Bagshaw and D. Cherny, Biochem. Soc. Trans., 2006, 34, 979-982.
10 B. F. Cravatt, A. T. Wright and J. W. Kozarich, Annu. Rev. Biochem., 2008, 77, 383-414.

11 M. Fernández-Suárez and A. Y. Ting, Nat. Rev. Mol. Cell Biol., 2008, 9, 929-943.

12 Q. Wang, T. Lin, L. Tang, J. E. Johnson and M. G. Finn, Angew. Chem., Int. Ed., 2002, 41, 459-462.

13 J. C. Smith, K.-B. Lee, Q. Wang, M. G. Finn, J. E. Johnson, M. Mrksich and C. A. Mirkin, Nano Lett., 2003, 3, 883-886.

14 D. W. P. M. Löwik, I. O. Shklyarevskiy, L. Ruizendaal, P. C. M. Christianen, J. C. Maan and J. C. M. van Hest, Adv. Mater., 2007, 19, 1191-1195.

15 K. L. Christman, R. M. Broyer, Z. P. Tolstyka and H. D. Maynard, J. Mater. Chem., 2007, 17, 2021.

16 M. J. Abedin, L. Liepold, P. Suci, M. Young and T. Douglas, J. Am. Chem. Soc., 2009, 131, 4346-4354.

17 L. S. Witus and M. B. Francis, Acc. Chem. Res., 2011, 44, 774-783.

18 M.-L. Chen, A. K. Adak, N.-C. Yeh, W.-B. Yang, Y.-J. Chuang, C.-H. Wong, K.-C. Hwang, J.-R. R. Hwu, S.-L. Hsieh and C.-C. Lin, Angew. Chem., Int. Ed., 2008, 47, 8627-8630.

19 A. Hucknall, D. H. Kim, S. Rangarajan, R. T. Hill, W. M. Reichert and A. Chilkoti, Adv. Mater., 2009, 21, 1968-1971.

20 Y.-L. Huang and C.-Y. Wu, Expert Rev. Vaccines, 2010, 9, 1257-1274.

21 S. Aggarwal, Nat. Biotechnol., 2010, 28, 1165-1171.

22 A. Mullard, Nat. Rev. Drug Discovery, 2013, 12, 329-332.

23 Y.-L. Huang, J.-T. Hung, S. K. C. Cheung, H.-Y. Lee, K.-C. Chu, S.-T. Li, Y.-C. Lin, C.-T. Ren, T.-J. R. Cheng, T.-L. Hsu, A. L. Yu, C.-Y. Wu and C.-H. Wong, Proc. Natl. Acad. Sci. U. S. A., 2013, 110, 2517-2522.

24 R. Adamo, A. Nilo, B. Castagner, O. Boutureira, F. Berti and G. J. L. Bernardes, Chem. Sci., 2013, 4, 2995-3008.

25 C. Gruttner, K. Muller and J. Teller, IEEE Trans. Magn., 2013, 49, 172-176.

26 A. Sinz, J. Mass Spectrom., 2003, 38, 1225-1237.

27 M. J. Hinner and K. Johnsson, Curr. Opin. Chem. Biol., 2010, 21, 766-776.

28 G. T. Hermanson, Bioconjugate Techniques, Academic Press, 2008.

29 F. H. Westheimer and D. E. Schmidt, Biochemistry, 1971, 10, 1249-1253.

30 G. W. Anderson, J. E. Zimmerman and F. M. Callahan, J. Am. Chem. Soc., 1964, 86, 1839-1842.

31 A. Lowe, Proc. R. Soc. Med., 1970, 63, 367-368.

32 A. H. Coons, H. J. Creech, R. N. Jones and E. Berliner, J. Immunol., 1942, 45, 159-170.

33 M. E. Annunziato, U. S. Patel, M. Ranade and P. S. Palumbo, Bioconjugate Chem., 1993, 4, 212-218.

34 H. J. Creech and R. N. Jones, J. Am. Chem. Soc., 1941, 63, 1670-1673.

35 L. Goodfriend and A. H. Sehon, Can. J. Biochem., 1958, 36, 1177-1184.

36 A. Rifai and S. S. Wong, J. Immunol. Methods, 1986, 94, 25-30.

37 A. Todrick and E. Walker, Biochem. J., 1937, 31, 297-298. 
38 P. Edman, Acta Chem. Scand., 1950, 4, 283-293.

39 D. Podhradský, Ĺ. Drobnica and P. Kristian, Experientia, 1979, 35, 154-155.

40 R. J. Seiwald, J. L. Riggs, J. H. Burckhalter, C. M. Downs and T. G. Metcalf, Am. J. Pathol., 1958, 34, 1081-1097.

41 R. P. Haugland, Molecular probes, in Handbook of fluorescent probes and research chemicals, 1996.

42 J. Tuls, L. Geren and F. Millett, J. Biol. Chem., 1989, 264, 16421-16425.

43 L. D. Burtnick, Biochim. Biophys. Acta, 1984, 791, 57-62.

44 M. Miki, Eur. J. Biochem., 1987, 164, 229-235.

45 M. Miki, J. Biochem., 1989, 106, 651-655.

46 A. Bellelli, R. Ippoliti, M. Brunori, Z. Kam, M. Benveniste, F. Emmanuel, E. Turpin, A. Alfsen and J. P. Frénoy, Biochem. Biophys. Res. Commun., 1990, 169, 602-609.

47 R. E. Dedmon, A. W. Holmes and F. Deinhardt, J. Bacteriol., 1965, 89, 734-739.

48 L. Amante, A. Ancona and L. Forni, J. Immunol. Methods, 1972, 1, 289-301.

49 A. F. Barbet and T. C. McGuire, Proc. Natl. Acad. Sci. U. S. A., 1978, 75, 1989-1993.

50 M. Schultzberg, J. M. Lundberg, T. Ho kfelt, L. Terenius, J. Brandt, R. P. Elde and M. Goldstein, Neuroscience, 1978, 3, 1169-1186.

51 W. P. Carney, R. H. Rubin, R. A. Hoffman, W. P. Hansen, K. Healey and M. S. Hirsch, J. Immunol., 1981, 126, 2114-2116.

52 A. Varadarajan, R. M. Sharkey, D. M. Goldenberg and M. F. Hawthorne, Bioconjugate Chem., 1991, 2, 102-110.

53 R. Pei, M. Arjomand-Shamsai, C. T. Deng, A. Cesbron, J. D. Bignon and J. H. Lee, Tissue Antigens, 1993, 41, 200-203.

54 M. Edidin, Y. Zagyansky and T. Lardner, Science, 2003, 191, 466-468.

55 K. K. Huang and C. R. C. Cantor, J. Mol. Biol., 1972, 67, 265-275.

56 S. H. Blose, J. Cell Biol., 1980, 86, 608-615.

57 S. Ohkuma, Y. Moriyama and T. Takano, Proc. Natl. Acad. Sci. U. S. A., 1982, 79, 2758-2762.

58 S. J. Morrison, H. D. Hemmati, A. M. Wandycz and I. L. Weissman, Proc. Natl. Acad. Sci. U. S. A., 1995, 92, 10302-10306.

59 C. A. Gabel, E. M. Eddy and B. M. Shapiro, J. Cell Biol., 1979, 82, 742-754.

60 P. Rosa-Neto, B. Wängler, L. Iovkova, G. Boening, A. Reader, K. Jurkschat and E. Schirrmacher, ChemBioChem, 2009, 10, 1321-1324.

61 K. E. Linder, M. D. Wen, D. P. Nowotnik, M. F. Malley, J. Z. Gougoutas, A. D. Nunn and W. C. Eckelman, Bioconjugate Chem., 1991, 2, 160-170.

62 C. F. Meares, M. J. McCall, D. T. Reardan, D. A. Goodwin, C. I. Diamanti and M. McTigue, Anal. Biochem., 1984, 142, 68-78.

63 S. S. Mirzadeh, M. W. M. Brechbiel, R. W. R. Atcher and O. A. O. Gansow, Bioconjugate Chem., 1989, 1, 59-65.

64 D. S. Wilbur, M.-K. Chyan, D. K. Hamlin, B. B. Kegley, R. Nilsson, B. E. B. Sandberg and M. Brechbiel, Bioconjugate Chem., 2002, 13, 1079-1092.
65 H. Shi, X. He, K. Wang, Y. Yuan, K. Deng, J. Chen and W. Tan, J. Nanomed. Nanotechnol., 2007, 3, 266-272.

66 M. Roederer, Conjugation of monoclonal antibodies, 2004, http://drmr.com/abcon/index.html.

67 E. C. E. Butcher and I. L. I. Weissman, J. Immunol. Methods, 1979, 37, 97-108.

68 A. M. Landel, Anal. Biochem., 1976, 73, 280-289.

69 D. S. Wilbur, M.-K. Chyan, H. Nakamae, Y. Chen, D. K. Hamlin, E. B. Santos, B. T. Kornblit and B. M. Sandmaier, Bioconjugate Chem., 2012, 23, 409-420.

70 P. R. Banks and D. M. Paquette, Bioconjugate Chem., 1995, 6, 447-458.

71 M. B. Smith and J. March, March's Advanced Organic Chemistry, John Wiley \& Sons, 2007.

72 G. P. Smith, Bioconjugate Chem., 2006, 17, 501-506.

73 O. Mäkelä, Immunology, 1966, 10, 81-86.

74 J. M. Becker, M. Wilchek and E. Katchalski, Proc. Natl. Acad. Sci. U. S. A., 1971, 68, 2604-2607.

75 M. Brinkley, Bioconjugate Chem., 1991, 3, 2-13.

76 G. W. Anderson, J. E. Zimmerman and F. M. Callahan, J. Am. Chem. Soc., 1963, 85, 3039.

77 G. W. Anderson, F. M. Callahan and J. E. Zimmerman, J. Am. Chem. Soc., 1967, 89, 178.

78 W.-C. Yang, H. Mirzaei, X. Liu and F. E. Regnier, Anal. Chem., 2006, 78, 4702-4708.

79 F. E. Regnier and S. Julka, Proteomics, 2006, 6, 3968-3979.

80 Y. Ishikawa, Y. Yamamoto, M. Otsubo, S. M. Theg and N. Tamura, Biochemistry, 2002, 41, 1972-1980.

81 B. Holmquist, S. Blumberg and B. L. Vallee, Biochemistry, 1976, 15, 4675-4680.

82 S. Blumberg and B. L. Vallee, Biochemistry, 1975, 14, 2410-2419.

83 M. D. Leavell, P. Novak, C. R. Behrens, J. S. Schoeniger and G. H. Kruppa, J. Am. Soc. Mass Spectrom., 2004, 15, 1604-1611.

84 P. Cuatrecasas and I. Parikh, Biochemistry, 1972, 11, 2291-2299.

85 B. T. Miller, T. J. Collins, G. T. Nagle and A. Kurosky, J. Biol. Chem., 1992, 267, 5060-5069.

86 B. T. Miller and A. Kurosky, Biochem. Biophys. Res. Commun., 1993, 196, 461-467.

87 B. T. Miller, Biochem. Biophys. Res. Commun., 1996, 218, 377-382.

88 B. T. Miller, T. J. Collins, M. E. Rogers and A. Kurosky, Peptides, 1997, 18, 1585-1595.

89 S. Mädler, C. Bich, D. Touboul and R. Zenobi, J. Mass Spectrom., 2009, 44, 694-706.

90 C. L. Swaim, J. B. Smith and D. L. Smith, Mikrochim. Acta, 2004, 15, 736-749.

91 J. S. Smith, B. T. Miller, S. L. Knock and A. Kurosky, Anal. Biochem., 1991, 197, 247-253.

92 S. Kalkhof and A. Sinz, Anal. Bioanal. Chem., 2008, 392, 305-312.

93 A. J. Lomant and G. Fairbanks, J. Mol. Biol., 1976, 104, 243-261.

94 J. V. Staros, R. W. Wright and D. M. Swingle, Anal. Biochem., 1986, 156, 220-222. 
95 N. Abello, H. A. M. Kerstjens, D. S. Postma and R. Bischoff, J. Proteome Res., 2007, 6, 4770-4776.

96 A. W. Yem, H. A. Zurcher-Neely, K. A. Richard, N. D. Staite, R. L. Heinrikson and M. R. Deibel, J. Biol. Chem., 1989, 264, 17691-17697.

97 V. C. Lombardi and D. A. Schooley, Anal. Biochem., 2004, 331, 40-45.

98 M. Morpurgo, E. A. Bayer and M. Wilchek, J. Biochem. Biophys. Methods, 1999, 38, 17-28.

99 M. Cooper, A. Ebner, M. Briggs, M. Burrows, N. Gardner, R. Richardson and R. West, J. Fluoresc., 2004, 14, 145-150.

100 Y. Deng, Z. Hou, L. Wang, C. Cherian, J. Wu, A. Gangjee and L. H. Matherly, Mol. Pharmacol., 2008, 73, 1274-1281.

101 D. Kahne and W. C. Still, J. Am. Chem. Soc., 1988, 110, 7529-7534.

102 J. M. Becker and M. Wilchek, Biochim. Biophys. Acta, 1972, 264, 165-170.

103 M. Trester-Zedlitz, K. Kamada, S. K. Burley, D. Fenyö, B. T. Chait and T. W. Muir, J. Am. Chem. Soc., 2003, 125, 2416-2425.

104 R. Kluger and A. Alagic, Bioinorg. Chem., 2004, 32, 451-472.

105 M. A. Trakselis, S. C. Alley and F. T. Ishmael, Bioconjugate Chem., 2005, 16, 741-750.

106 X. Tang, G. R. Munske, W. F. Siems and J. E. Bruce, Anal. Chem., 2005, 77, 311-318.

107 J. D. Chavez, N. L. Liu and J. E. Bruce, J. Proteome Res., 2011, 10, 1528-1537.

108 M. Q. Müller, F. Dreiocker, C. H. Ihling, M. Schäfer and A. Sinz, Anal. Chem., 2010, 82, 6958-6968.

109 J. W. Back, L. de Jong, A. O. Muijsers and C. G. de Koster, J. Mol. Biol., 2003, 331, 303-313.

110 M. M. Young, N. Tang, J. C. Hempel, C. M. Oshiro, E. W. Taylor, I. D. Kuntz, B. W. Gibson and G. Dollinger, Proc. Natl. Acad. Sci. U. S. A., 2000, 97, 5802-5806.

111 S. Avrameas, Immunochemistry, 1969, 6, 43-52.

112 B. F. Erlanger, Pharmacol. Rev., 1973, 25, 271-280.

113 M. C. Garnett, Adv. Drug Delivery Rev., 2001, 53, 171-216.

114 D. M. Schulz, C. Ihling, G. M. Clore and A. Sinz, Biochemistry, 2004, 43, 4703-4715.

115 S. Takeda, S. Tsukiji, H. Ueda and T. Nagamune, Org. Biomol. Chem., 2008, 6, 2187-2194.

116 N. K. Damle, Nat. Biotechnol., 2008, 26, 884-885.

117 S. C. Alley, N. M. Okeley and P. D. Senter, Curr. Opin. Chem. Biol., 2010, 14, 529-537.

118 S. Webb, Nat. Biotechnol., 2011, 29, 297-298.

119 W.-D. Janthur, N. Cantoni and C. Mamot, Int. J. Mol. Sci., 2012, 13, 16020-16045.

120 R. S. Zolot, S. Basu and R. P. Million, Nat. Rev. Drug Discovery, 2013, 12, 259-260.

121 P. M. Abdella, P. K. Smith and G. P. Royer, Biochem. Biophys. Res. Commun., 1979, 87, 734-742.

122 D. A. Zarling, A. Watson and F. H. Bach, J. Immunol., 1980, 124, 913-920.

123 R. J. Smith, R. A. Capaldi, D. Muchmore and F. Dahlquist, Biochemistry, 1978, 17, 3719-3723.
124 R. Reents, D. A. Jeyaraj and H. Waldmann, Drug Discovery Today, 2002, 7, 71-76.

125 G. Leriche, L. Chisholm and A. Wagner, Bioorg. Med. Chem., 2012, 20, 571-582.

126 Y. Jin Lee, Mol. BioSyst., 2008, 4, 816-823.

127 A. Sinz, S. Kalkhof and C. Ihling, J. Am. Soc. Mass Spectrom., 2005, 16, 1921-1931.

128 M. Wilchek and E. A. Bayer, Methods Enzymol., 1990, 184, 123-138.

129 E. A. Bayer and M. Wilchek, Methods Enzymol., 1990, 184, 138-160.

130 X. Chen, K. Muthoosamy, A. Pfisterer, B. Neumann and T. Weil, Bioconjugate Chem., 2012, 23, 500-508.

131 E. R. Goldman, E. D. Balighian, H. Mattoussi, M. K. Kuno, J. M. Mauro, P. T. Tran and G. P. Anderson, J. Am. Chem. Soc., 2002, 124, 6378-6382.

132 M. R. Lockett, M. F. Phillips, J. L. Jarecki, D. Peelen and L. M. Smith, Langmuir, 2008, 24, 69-75.

133 M. Yang, R. L. Teeuwen, M. Giesbers, J. Baggerman, A. Arafat, F. A. de Wolf, J. C. van Hest and H. Zuilhof, Langmuir, 2008, 24, 7931-7938.

134 A. E. Bolton and W. M. Hunter, Biochem. J., 1973, 133, 529-539.

135 J. J. Langone, Methods Enzymol., 1980, 70, 221-247.

136 P. Anderson, Methods Enzymol., 1986, 119, 281-283.

137 M. Glaser, V. A. Carroll, D. R. Collingridge, E. O. Aboagye, P. Price, R. Bicknell, A. L. Harris, S. K. Luthra and F. Brady, J. Labelled Compd. Radiopharm., 2002, 45, 1077-1090.

138 J. Russell, J. A. O’Donoghue, R. Finn, J. Koziorowski, S. Ruan, J. L. Humm and C. C. Ling, J. Nucl. Med., 2002, 43, 671-677.

139 D. R. Collingridge, V. A. Carroll, M. Glaser and E. O. Aboagye, Cancer Res., 2002, 62, 5912-5919.

140 J. J. Park, T. S. Lee, J. H. Kang, R. Song and G. J. Cheon, Appl. Radiat. Isot., 2011, 69, 56-62.

141 T. H. Ji and I. Ji, Anal. Biochem., 1982, 121, 286-289.

142 P. L. Ross, Mol. Cell. Proteomics, 2004, 3, 1154-1169.

143 L. R. Zieske, J. Exp. Bot., 2006, 57, 1501-1508.

144 K. Aggarwal, L. H. Choe and K. H. Lee, Briefings Funct. Genomics Proteomics, 2006, 5, 112-120.

145 H. Mirzaei and F. Regnier, Anal. Chem., 2006, 78, 4175-4183.

146 R. F. Borch, M. D. Bernstein and H. D. Durst, J. Am. Chem. Soc., 1971, 93, 2897-2904.

147 G. R. Gray, Arch. Biochem. Biophys., 1974, 163, 426-428.

148 J. C. Gildersleeve, O. Oyelaran, J. T. Simpson and B. Allred, Bioconjugate Chem., 2008, 19, 1485-1490.

149 B. A. Schwartz and G. R. Gray, Arch. Biochem. Biophys., 1977, 181, 542-549.

150 R. T. Lee and Y. C. Lee, Biochemistry, 1980, 19, 156-163.

151 T. Freire, S. Bay, S. Vichier-Guerre, R. Lo-Man and C. Leclerc, Mini-Rev. Med. Chem., 2006, 6, 1357-1373.

152 S. Y. Wong, Curr. Opin. Struct. Biol., 1995, 5, 599-604.

153 P. H. Seeberger and D. B. Werz, Nature, 2007, 446, 1046-1051.

154 L. Cipolla, F. Peri and C. Airoldi, Anti-Cancer Agents Med. Chem., 2008, 8, 92-121. 
155 S. B. Oppenheimer, M. Alvarez and J. Nnoli, Acta Histochem., 2008, 110, 6-13.

156 D. C. Tahmassebi and T. Sasaki, J. Org. Chem., 1998, 63, 728-731.

157 M. D. Bentley, M. J. Roberts and J. M. Harris, J. Pharm. Sci., 1998, 87, 1446-1449.

158 L. Tao, G. Mantovani, F. Lecolley and D. M. Haddleton, J. Am. Chem. Soc., 2004, 126, 13220-13221.

159 R. Mallik, T. Jiang and D. S. Hage, Anal. Chem., 2004, 76, 7013-7022.

160 R. Tripathi, S. Verma, J. Pandey and V. Tiwari, Curr. Org. Chem., 2008, 12, 1093-1115.

161 J. McFarland and M. Francis, J. Am. Chem. Soc., 2005, 127, 13490-13491.

162 G. Weber, Biochem. J., 1952, 51, 155-167.

163 B. S. Hartley and V. Massey, Biochim. Biophys. Acta, 1956, 21, 58-70.

164 W. R. Gray, Methods Enzymol., 1967, 11, 469-475.

165 C. Lefevre, H. C. Kang, R. P. Haugland, N. Malekzadeh, S. Arttamangkul and R. P. Haugland, Bioconjugate Chem., 1996, 7, 482-489.

166 J. A. J. Titus, R. R. Haugland, S. O. S. Sharrow and D. M. D. Segal, J. Immunol. Methods, 1981, 50, 193-204.

167 N. Seiler, Methods Biochem. Anal., 1970, 18, 259-337.

168 Methods in Molecular Biology, Humana Press, Totowa, NJ, 2009, vol. 588, pp. 43-48.

169 W. R. Gray, Methods Enzymol., 1967, 11, 139-151.

170 C. Gros and B. Labouesse, Eur. J. Biochem., 1969, 7, 463-470.

171 R. E. Boyle, J. Org. Chem., 1966, 31, 3880-3882.

172 J. K. Lin and J. Y. Chang, Anal. Chem., 1975, 47, 1634-1638.

173 R. A. Evangelista, A. Pollak, B. Allore, A. F. Templeton, R. C. Morton and E. P. Diamandis, Clin. Biochem., 1988, 21, 173-178.

174 A. Andreoni, G. Bottiroli, A. Colasanti, M. C. Giangarè, P. Riccio, G. Roberti and P. Vaghi, J. Biochem. Biophys. Methods, 1994, 29, 157-172.

175 B. M. Gorovits and P. M. Horowitz, J. Biol. Chem., 1995, 270, 13057-13062.

176 D. J. Herzig, A. W. Rees and R. A. Day, Biopolymers, 1964, 2, 349-360.

177 A. M. Gold, Biochemistry, 1965, 4, 897-901.

178 R. S. Tipson, J. Org. Chem., 1944, 9, 235-241.

179 R. K. Crossland, W. E. Wells and V. J. Shiner, J. Am. Chem. Soc., 1971, 93, 4217-4219.

180 K. Nilsson and K. Mosbach, Biochem. Biophys. Res. Commun., 1981, 102, 449-457.

181 Z. Ma, C. Gao, J. Ji and J. Shen, Eur. Polym. J., 2002, 38, 2279-2284.

182 K. Pugdee, Y. Shibata, N. Yamamichi, H. Tsutsumi, M. Yoshinari, Y. Abiko and T. Hayakawa, Dent. Mater. J., 2007, 26, 647-655.

183 C. Delgado, J. N. Patel, G. E. Francis and D. Fisher, Biotechnol. Bioprocess Eng., 1990, 12, 119-128.

184 G. E. Francis, D. Fisher, C. Delgado, F. Malik, A. Gardiner and D. Neale, Int. J. Hematol., 1998, 68, 1-18.
185 M. J. Roberts, M. D. Bentley and J. M. Harris, Adv. Drug Delivery Rev., 2012, 64, 116-127.

186 H.-J. Gais and S. Ruppert, Tetrahedron Lett., 1995, 36, 3837-3838.

187 H. T. F. Sanger, Biochem. J., 1951, 49, 481-490.

188 H. N. Eisen, S. Belman and M. E. Carsten, J. Am. Chem. Soc., 1953, 75, 4583-4585.

189 S. Shaltiel, Biochem. Biophys. Res. Commun., 1967, 29, 178-183.

190 K. Imai and Y. Watanabe, Anal. Chim. Acta, 1981, 130, 377-383.

191 A. A. Elbashir and F. Suliman, Appl. Spectrosc., 2011, 46, 222-241.

192 S. Uchiyama, T. Santa, N. Okiyama, T. Fukushima and K. Imai, Biomed. Chromatogr., 2001, 15, 295-318.

193 T. Santa, T. Fukushima, T. Ichibangase and K. Imai, Biomed. Chromatogr., 2008, 22, 343-353.

194 P. Smialowski and D. Frishman, in Data Mining Techniques for the Life Sciences, Humana Press, 2010, vol. 609, pp. 385-400, DOI: 10.1007/978-1-60327-241-4_22.

195 D. A. Sutton, S. E. Drewes and U. Welz, Biochem. J., 1972, 130, 589-595.

196 M. Lesniak, J. Gliemann, J. Roth, B. R. Easter, D. A. Sutton and S. E. Drewes, Hoppe-Seyler's Z. Physiol. Chem., 1979, 360, 467-472.

197 D. A. Sutton, B. R. Easter and S. E. Drewes, Hoppe-Seyler's Z. Physiol. Chem., 1976, 357, 971-976.

198 G. Losse and G. Mützler, Z. Chem., 1981, 21, 394-403.

199 D. L. Ladd and R. A. Snow, Anal. Biochem., 1993, 210, 258-261.

200 M. J. Hunter and M. L. Ludwig, J. Am. Chem. Soc., 1962, 84, 3491-3504.

201 R. R. Traut, A. Bollen, T.-T. Sun, J. W. B. Hershey, J. Sundberg and L. R. Pierce, Biochemistry, 1973, 12, 3266-3273.

202 M. Nomura, Science, 1973, 179, 864-873.

203 L. Wofsy and S. J. Singer, Biochemistry, 1963, 2, 104-116.

204 H. J. H. Schramm, Hoppe-Seyler's Z. Physiol. Chem., 1975, 356, 1375-1379.

205 H. Bozler, K. D. Jany and G. Pfleiderer, Biochim. Biophys. Acta, 1983, 749, 238-243.

206 Y. C. Lee, C. P. Stowell and M. J. Krantz, Biochemistry, 1976, 15, 3956-3963.

207 R. R. Mehlhorn, M. M. Swanson, L. L. Packer and P. P. Smith, Arch. Biochem. Biophys., 1980, 204, 471-476.

208 J. B. J. Denny and R. M. R. Roberts, J. Biol. Chem., 1982, 257, 2460-2468.

209 F. T. Wood, M. M. Wu and J. C. Gerhart, Anal. Biochem., 1975, 69, 339-349.

210 D. E. Wright, EP0539167, 1993, https://patentscope.wipo. int/search/en/detail.jsf?docId=EP12397580.

211 K. Ampon and G. E. Means, Biotechnol. Bioeng., 1988, 32, 689-697.

212 J. I. Gavrilyuk, U. Wuellner and C. F. Barbas, Bioorg. Med. Chem. Lett., 2009, 19, 1421-1424.

213 J. I. Gavrilyuk, U. Wuellner, S. Salahuddin, R. K. Goswami, S. C. Sinha and C. F. Barbas III, Bioorg. Med. Chem. Lett., 2009, 19, 3716-3720. 
214 L. F. Tietze, M. Arlt, M. Beller, K.-H. Glüsenkamp, E. Jähde and M. F. Rajewsky, Chem. Ber., 1991, 124, 1215-1221.

215 L. F. Tietze, C. Schroeter, S. Gabius, U. Brinck, A. GoerlachGraw and H. J. Gabius, Bioconjugate Chem., 1991, 2, 148-153.

216 F. Wurm, C. Dingels, H. Frey and H.-A. Klok, Biomacromolecules, 2012, 13, 1161-1171.

217 F. Wurm, T. Steinbach and H.-A. Klok, Chem. Commun., 2013, 49, 7815-7817.

218 D. Blakeslee and M. G. Baines, J. Immunol. Methods, 1976, 13, 305-320.

219 D. Blakeslee, J. Immunol. Methods, 1977, 17, 361-364.

220 A. Abuchowski, T. Es, N. C. Palczuk and F. F. Davis, J. Biol. Chem., 1977, 252, 3578-3581.

221 A. Abuchowski, J. R. McCoy, N. C. Palczuk, T. Es and F. F. Davis, J. Biol. Chem., 1977, 252, 3582-3586.

222 A. Matsushima, Chem. Lett., 1980, 773-776.

223 S. S. Arpicco, F. F. Dosio, A. A. Bolognesi, C. C. Lubelli, P. P. Brusa, B. B. Stella, M. M. Ceruti and L. L. Cattel, Bioconjugate Chem., 2002, 13, 757-765.

224 Y. Ikeda, J. Katamachi, H. Kawasaki and Y. Nagasaki, Bioconjugate Chem., 2013, 24, 1824-1827.

225 F. R. Gurd, Methods Enzymol., 1971, 25, 424-438.

226 I. Forristal, J. Sulfur Chem., 2005, 26, 163-185.

227 E. Valero, S. Tambalo, P. Marzola, M. Ortega-Muñoz, F. J. Lopez-Jaramillo, F. Santoyo-Gonzalez, J. de Dios López, J. J. Delgado, J. J. Calvino, R. Cuesta, J. M. Domínguez-Vera and N. Gálvez, J. Am. Chem. Soc., 2011, 133, 4889-4895.

228 A. Megia-Fernandez, F. Hernandez-Mateo and F. SantoyoGonzalez, Org. Biomol. Chem., 2013, 11, 2586-2596.

229 R. L. Lundblad, Chemical Reagents for Protein Modification, CRC Press, 3rd edn, 2010.

230 M.-P. Brun and L. Gauzy-Lazo, Antibody-Drug conjugates, 2013, 1045, 173-187.

231 S. Mädler, M. Seitz, J. Robinson and R. Zenobi, Mikrochim. Acta, 2010, 21, 1775-1783.

232 B. T. Miller, M. E. Rogers, J. S. Smith and A. Kurosky, Anal. Biochem., 1994, 219, 240-248.

233 S. Mädler, S. Gschwind and R. Zenobi, Role of arginine in chemical cross-linking with $\mathrm{N}$-hydroxysuccinimide esters, 2010, vol. 398.

234 F. Brotzel and H. Mayr, Org. Biomol. Chem., 2007, 5, 3814-3820.

235 D. Gilis, S. Massar, N. J. Cerf and M. Rooman, Genome Biol., 2001, 2, 1-12.

236 M. N. Fodje and S. Al-Karadaghi, Protein Eng., 2002, 15, 353-358.

237 S. J. Sirk, T. Olafsen, B. Barat, K. B. Bauer and A. M. Wu, Bioconjugate Chem., 2008, 19, 2527-2534.

238 J. J. Gorman, G. L. Corino and S. J. Mitchell, Eur. J. Biochem., 1987, 168, 169-179.

239 J. A. Burns, J. C. Butler, J. Moran and G. M. Whitesides, J. Org. Chem., 1991, 56, 2648-2650.

240 E. B. Getz, M. Xiao, T. Chakrabarty, R. Cooke and P. R. Selvin, Anal. Biochem., 1999, 273, 73-80.

241 R. E. Hansen and J. R. Winther, Anal. Biochem., 2009, 394, 147-158.
242 K. Tyagarajan, E. Pretzer and J. E. Wiktorowicz, Electrophoresis, 2003, 24, 2348-2358.

243 D. E. Shafer, J. K. Inman and A. Lees, Anal. Biochem., 2000, 282, 161-164.

244 D. R. Goddard and L. Michaelis, J. Biol. Chem., 1935, 112, 361-371.

245 N. Lundell and T. Schreitmüller, Anal. Biochem., 1999, 266, 31-47.

246 F. R. Gurd, Methods Enzymol., 1972, 25, 424-438.

247 M. L. Nielsen, M. Vermeulen, T. Bonaldi, J. Cox, L. Moroder and M. Mann, Nat. Methods, 2008, 5, 459-460.

248 B. I. Gerwin, J. Biol. Chem., 1967, 242, 451-456.

249 J. R. Kim, H. W. Yoon, K. S. Kwon, S. R. Lee and S. G. Rhee, Anal. Biochem., 2000, 283, 214-221.

250 N. J. Davis and S. L. Flitsch, Tetrahedron Lett., 1991, 32, 6793-6796.

251 D. Macmillan, R. Bill, K. Sage, D. Fern and S. Flitsch, Chem. Biol., 2001, 8, 133-145.

252 R. S. Swanwick, A. M. Daines, L.-H. Tey, S. L. Flitsch and R. K. Allemann, ChemBioChem, 2005, 6, 1338-1340.

253 J. F. Mackworth, Biochem. J., 1948, 42, 82-90.

254 P. I. Clark and G. Lowe, J. Am. Chem. Soc., 1977, 24, 923-924.

255 P. I. Clark and G. Lowe, Eur. J. Biochem., 1978, 84, 293-299.

256 R. W. Furlanetto and E. T. Kaiser, J. Am. Chem. Soc., 1970, 92, 6980-6982.

257 R. W. Furlanetto, M. Mochizuki and E. T. Kaiser, Biochem. Biophys. Res. Commun., 1974, 58, 192-196.

258 K. J. Schwartz, Y. Nakagawa and E. T. Kaiser, J. Am. Chem. Soc., 1976, 98, 6369-6378.

259 E. Kaiser and D. Lawrence, Science, 1984, 226, 505-511.

260 E. Friedmann and D. H. Marrian, Br. J. Pharmacol., 1949, 4, 105-108.

261 E. Friedmann, Biochim. Biophys. Acta, 1952, 9, 65-75.

262 T. Kajfež, B. Kamenar, V. Piližota and D. Fleš, Croat. Chem. Acta, 2003, 76, 343-346.

263 S. G. Bodige, M. A. Méndez-Rojas and W. H. Watson, J. Chem. Crystallogr., 1999, 29, 57-66.

264 D. G. Smyth, O. O. Blumenfeld and W. Konigsberg, Biochem. J., 1964, 91, 589-595.

265 R. D. Brown and K. S. Matthews, J. Biol. Chem., 1979, 254, 5128-5134.

266 Y. Ishii and S. S. Lehrer, Biophys. J., 1986, 50, 75-80.

267 C.-W. Wu, L. R. Yarbrough and F. Y. Wu, Biochemistry, 1976, 15, 2863-2868.

268 T. Climent, R. González-Luque and M. Merchán, J. Phys. Chem. A, 2003, 107, 6995-7003.

269 P. Schelté, C. Boeckler, B. Frisch and F. Schuber, Bioconjugate Chem., 2000, 11, 118-123.

270 S. Yoshitake, Y. Yamada, E. Ishikawa and R. Masseyeff, Eur. J. Biochem., 1979, 101, 395-399.

271 S. Yoshitake, M. Imagawa, E. Ishikawa, Y. Niitsu, I. Urushizaki, M. Nishiura, R. Kanazawa, H. Kurosaki, S. Tachibana and N. Nakazawa, J. Biochem., 1982, 92, 1413-1424.

272 S. Hashida, M. Imagawa, S. Inoue, K. H. Ruan and E. Ishikawa, J. Appl. Biochem., 1984, 6, 56-63. 
273 S. S. Ghosh, P. M. Kao, A. W. McCue and H. L. Chappelle, Bioconjugate Chem., 1990, 1, 71-76.

274 N. Dickgreber, P. Stoitzner, Y. Bai, K. M. Price, K. J. Farrand, K. Manning, C. E. Angel, P. R. Dunbar, F. Ronchese, J. D. Fraser, B. T. Bäckström and I. F. Hermans, J. Immunol., 2009, 182, 1260-1269.

275 T. Hu and Z. Su, Biotechnol. Lett., 2002, 24, 275-278.

276 K. Kafi, D. J. Betting, R. E. Yamada, M. Bacica, K. K. Steward and J. M. Timmerman, Mol. Immunol., 2009, 46, 448-456.

277 G. K. Ehrlich, H. Michel, T. Truitt and W. Riboulet, Bioconjugate Chem., 2013, 24, 2015-2024.

278 F. Kratz, A. Warnecke, K. Scheuermann, C. Stockmar, J. Schwab, P. Lazar, P. Drückes, N. Esser, J. Drevs, D. Rognan, C. Bissantz, C. Hinderling, G. Folkers, I. Fichtner and C. Unger, J. Med. Chem., 2002, 45, 5523-5533.

279 A. Warnecke, I. Fichtner, G. Sass and F. Kratz, Arch. Pharm., 2007, 340, 389-395.

280 V. Kersemans, B. Cornelissen, M. D. Minden, J. Brandwein and R. M. Reilly, J. Nucl. Med., 2008, 49, 1546-1554.

281 D. J. Betting, K. Kafi, A. Abdollahi-Fard, S. A. Hurvitz and J. M. Timmerman, J. Immunol., 2008, 181, 4131-4140.

282 A. Alisio, J. Biol. Chem., 2004, 279, 26540-26545.

283 L. Giron-Monzon, J. Biol. Chem., 2004, 279, 49338-49345.

284 N. S. Green, E. Reisler and K. N. Houk, Protein Sci., 2008, 10, 1293-1304.

285 F. T. Ishmael, M. A. Trakselis and S. J. Benkovic, J. Biol. Chem., 2003, 278, 3145-3152.

286 O. V. Kovalenko, X. Yang, T. V. Kolesnikova and M. E. Hemler, Biochem. J., 2004, 377, 407-417.

287 Y. K. Kwon, J. Cell Biol., 2003, 163, 375-384.

288 M. A. Stalteri and S. J. Mather, Bioconjugate Chem., 1995, 6, 179-186.

289 E. E. Leary Swan, K. C. Popat and T. A. Desai, Biomaterials, 2005, 26, 1969-1976.

290 A. Misra and P. Dwivedi, Anal. Biochem., 2007, 369, 248-255.

291 G. Elia, Curr. Protein Pept. Sci., 2010, ch. 3. Unit 3.6.

292 Y. Oda, T. Nagasu and B. T. Chait, Nat. Biotechnol., 2001, 19, 379-382.

293 S. Khurana, M. Kennedy, L. R. King and H. Golding, J. Virol., 2005, 79, 6791-6800.

294 H. Golding, J. Biol. Chem., 2005, 280, 29570-29577.

295 F.-U. Gast, I. Franke, G. Meiss and A. Pingoud, J. Biotechnol., 2001, 87, 131-141.

296 I. H. Cho, E. H. Paek, H. Lee, J. W. Choi and S. H. Paek, Biotechnol. Bioprocess Eng., 2004, 9, 112-117.

297 G. Y. Kang, G. Y. Han, J. Y. Kang, I.-H. Cho, H.-H. Park, S.-H. Paek and T. S. Kim, Sens. Actuators, B, 2006, 117, 332-338.

298 I.-H. Cho, E.-H. Paek, H. Lee, J. Y. Kang, T. S. Kim and S.-H. Paek, Anal. Biochem., 2007, 365, 14-23.

299 A. Pompella, S. Dominici, C. Cambiaggi, J. Frank and H. K. Biesalski, Methods Mol. Biol., 2002, 196, 305-312.

300 J. M. Kuiper, R. Pluta, W. H. C. Huibers, F. Fusetti, E. R. Geertsma and B. Poolman, Protein Sci., 2009, 18, 1033-1041.
301 X. Chen, Y. Zhou, X. Peng and J. Yoon, Chem. Soc. Rev., 2010, 39, 2120.

302 Y. Zhang, V. S. Bhatt, G. Sun, P. G. Wang and A. F. Palmer, Bioconjugate Chem., 2008, 19, 2221-2230.

303 S. Ahlgren, A. Orlova, D. Rosik, M. Sandström, A. Sjöberg, B. Baastrup, O. Widmark, G. Fant, J. Feldwisch and V. Tolmachev, Bioconjugate Chem., 2008, 19, 235-243.

304 C. A. Boswell, J. Marik, M. J. Elowson, N. A. Reyes, S. Ulufatu, D. Bumbaca, V. Yip, E. E. Mundo, N. Majidy, M. Van Hoy, S. N. Goriparthi, A. Trias, H. S. Gill, S. P. Williams, J. R. Junutula, P. J. Fielder and L. A. Khawli, J. Med. Chem., 2013, 56, 9418-9426.

305 M. K. Han, P. Lin, D. Paek, J. J. Harvey, E. Fuior and J. R. Knutson, Biochemistry, 2002, 41, 3468-3476.

306 J. M. West, J. Biol. Chem., 2003, 279, 945-951.

307 F. Fernandes, L. M. S. Loura, R. Koehorst, R. B. Spruijt, M. A. Hemminga, A. Fedorov and M. Prieto, Biophys. J., 2004, 87, 344-352.

308 T. G. Nishino, K. Kitano, K. Kojima, T. Ogishima, A. Ito and S. Kitada, J. Biochem., 2007, 141, 889-895.

309 S. K. Wright and R. E. Viola, Anal. Biochem., 1998, 265, 8-14.

310 P. G. Mastroberardino, A. L. Orr, X. Hu, H. M. Na and J. T. Greenamyre, Free Radical Biol. Med., 2008, 45, 971-981.

311 G. Mantovani, F. Lecolley, L. Tao, D. M. Haddleton, J. Clerx, J. J. L. M. Cornelissen and K. Velonia, J. Am. Chem. Soc., 2005, 127, 2966-2973.

312 S. Matsui and H. Aida, J. Chem. Soc., Perkin Trans. 2, 1978, 1277-1280.

313 J. Kalia and R. T. Raines, Bioorg. Med. Chem. Lett., 2007, 17, 6286-6289.

314 L. M. Tedaldi, M. E. B. Smith, R. I. Nathani and J. R. Baker, Chem. Commun., 2009, 6583-6585.

315 D. D. Roberts, S. D. Lewis, D. P. Ballou, S. T. Olson and J. A. Shafer, Biochemistry, 1986, 25, 5595-5601.

316 M. E. B. Smith, F. F. Schumacher, C. P. Ryan, L. M. Tedaldi, D. Papaioannou, G. Waksman, S. Caddick and J. R. Baker, J. Am. Chem. Soc., 2010, 132, 1960-1965.

317 C. P. Ryan, M. E. B. Smith, F. F. Schumacher, D. Grohmann, D. Papaioannou, G. Waksman, F. Werner, J. R. Baker and S. Caddick, Chem. Commun., 2011, 47, 5452-5454.

318 L. Castañeda, A. Maruani, F. F. Schumacher, E. Miranda, V. Chudasama, K. A. Chester, J. R. Baker, M. E. B. Smith and S. Caddick, Chem. Commun., 2013, 49, 8187-8189.

319 J. Porath and R. Axén, Methods Enzymol., 1976, 44, 19-45.

320 M. S. Masri and M. Friedman, J. Protein Chem., 1988, 7, 49-54.

321 G. Houen and O. M. Jensen, J. Immunol. Methods, 1995, 181, 187-200.

322 M. Morpurgo, F. M. Veronese, D. Kachensky and J. M. Harris, Bioconjugate Chem., 1996, 7, 363-368.

323 D. C. Meadows and J. Gervay-Hague, Med. Res. Rev., 2006, 26, 793-814.

324 P. Bailon and C.-Y. Won, Expert Opin. Drug Delivery, 2009, 6, 1-16. 
325 F. Rusmini, Z. Zhong and J. Feijen, Biomacromolecules, 2007, 8, 1775-1789.

326 M. Ortega-Muñoz, J. Morales-Sanfrutos, A. Megia-Fernandez, F. J. Lopez-Jaramillo, F. Hernandez-Mateo and F. SantoyoGonzalez, J. Mater. Chem., 2010, 20, 7189-7196.

327 J. Morales-Sanfrutos, J. Lopez-Jaramillo, M. Ortega-Muñoz, A. Megia-Fernandez, F. Perez-Balderas, F. Hernandez-Mateo and F. Santoyo-Gonzalez, Org. Biomol. Chem., 2010, 8, 667-675.

328 F. J. Lopez-Jaramillo, M. Ortega-Muñoz, A. Megia-Fernandez, F. Hernandez-Mateo and F. Santoyo-Gonzalez, Bioconjugate Chem., 2012, 23, 846-855.

329 L. Li, S.-W. Tsai, A.-L. Anderson, D. A. Keire, A. A. Raubitschek and J. E. Shively, Bioconjugate Chem., 2002, 13, 110-115.

330 J. Morales-Sanfrutos, F. J. Lopez-Jaramillo, F. HernandezMateo and F. Santoyo-Gonzalez, J. Org. Chem., 2010, 75, 4039-4047.

331 F. Lopez-Jaramillo, F. Hernandez Mateo, F. Santoyo Gonzalez and E. Leung, Integr. Proteom., 2012, 16, 301-326.

332 H. Ovaa, et al., Angew. Chem., Int. Ed., 2003, 42, 3626-3629.

333 T. Posner, Chem. Ber., 1905, 38, 646-657.

334 C. E. Hoyle and C. N. Bowman, Angew. Chem., Int. Ed., 2010, 49, 1540-1573.

335 A. Dondoni and A. Marra, Chem. Soc. Rev., 2012, 41, 573-586.

336 N. Floyd, B. Vijayakrishnan, J. R. Koeppe and B. G. Davis, Angew. Chem., Int. Ed., 2009, 121, 7938-7942.

337 A. Dondoni, A. Massi, P. Nanni and A. Roda, Chem. - Eur. J., 2009, 15, 11444-11449.

338 L.-Z. Wu, Y.-B. Sheng, J.-B. Xie and W. Wang, J. Mol. Struct., 2008, 882, 101-106.

339 K. C. A. Garber and E. E. Carlson, ACS Chem. Biol., 2013, 8, 1671-1676.

340 R. M. Stolz and B. H. Northrop, J. Org. Chem., 2013, 78, 8105-8116.

341 L. Markey, S. Giordani and E. M. Scanlan, J. Org. Chem., 2013, 78, 4270-4277.

342 A. Massi and D. Nanni, Org. Biomol. Chem., 2012, 10, 3791-3807.

343 H. Bader, L. C. Cross, I. Heilbron and E. Jones, J. Chem. Soc., 1949, 619-623.

344 M. Lo Conte, S. Pacifico, A. Chambery, A. Marra and A. Dondoni, J. Org. Chem., 2010, 75, 4644-4647.

345 M. L. Conte, S. Staderini, A. Marra, M. Sanchez-Navarro, B. G. Davis and A. Dondoni, Chem. Commun., 2011, 47, 11086-11088.

346 M. W. Crankshaw and G. A. Grant, Curr. Protein Pept. Sci., 2001, ch. 15. unit 15.1.

347 M. L. Anson, J. Gen. Physiol., 1940, 23, 321-331.

348 G. L. Ellman, Arch. Biochem. Biophys., 1959, 82, 70-77.

349 G. L. Kenyon and T. W. Bruice, Methods Enzymol., 1977, 47, 407-430.

350 A. Fontana, E. Scoffone and C. A. Benassi, Biochemistry, 1968, 7, 980-986.

351 T. P. King, Y. Li and L. Kochoumian, Biochemistry, 1978, 17, 1499-1506.
352 W. M. Macindoe, A. H. van Oijen and G.-J. Boons, Chem. Commun., 1998, 847-848.

353 B. G. Davis, M. A. Maughan, M. P. Green, A. Ullman and J. B. Jones, Tetrahedron: Asymmetry, 2000, 11, 245-262.

354 D. P. Gamblin, P. Garnier, S. J. Ward, N. J. Oldham, A. J. Fairbanks and B. G. Davis, Org. Biomol. Chem., 2003, 1, 3642 .

355 B. G. Davis, Macromolecules, 2002, 102, 579-602.

356 Y. J. Zhao, Y. Q. Zhai, Z. G. Su and G. H. Ma, Polym. Adv. Technol., 2010, 21, 867-873.

357 D. P. Gamblin, P. Garnier, S. van Kasteren, N. J. Oldham, A. J. Fairbanks and B. G. Davis, Angew. Chem., Int. Ed., 2004, 116, 846-851.

358 G. J. L. Bernardes, D. P. Gamblin and B. G. Davis, Angew. Chem., Int. Ed., 2006, 118, 4111-4115.

359 G. J. L. Bernardes, J. P. Marston, A. S. Batsanov, J. A. K. Howard and B. G. Davis, Chem. Commun., 2007, 3145-3147.

360 M. Steiner, I. Hartmann, E. Perrino, G. Casi, S. Brighton, I. Jelesarov, G. J. Bernardes and D. Neri, Chem. Sci., 2013, 4, 297-302.

361 M. Kunstelj, K. Fidler, S. Skrajnar, M. Kenig, V. Smilović, M. Kusterle, S. Caserman, I. Zore, V. G. Porekar and S. Jevševar, Bioconjugate Chem., 2013, 24, 889-896.

362 G. J. L. Bernardes, E. J. Grayson, S. Thompson, J. M. Chalker, J. C. Errey, F. El Oualid, T. D. W. Claridge and B. G. Davis, Angew. Chem., Int. Ed., 2008, 47, 2244-2247.

363 K. Whiteman, C. Audette and S. D. Wilhelm, Bioconjugate Chem., 2009, 21, 84-92.

364 M. S. Rosendahl, D. H. Doherty, D. J. Smith, S. J. Carlson, E. A. Chlipala and G. N. Cox, Bioconjugate Chem., 2005, 16, 200-207.

365 M. T. Petersen, P. H. Jonson and S. B. Petersen, Protein Eng., 1999, 12, 535-548.

366 H. J. Leung, G. Xu, M. Narayan and H. A. Scheraga, J. Pept. Res., 2005, 65, 47-54.

367 T. Arakawa, S. J. Prestrelski, L. O. Narhi, T. C. Boone and W. C. Kenney, J. Protein Chem., 1993, 12, 525-531.

368 S. Brocchini, S. Balan, A. Godwin, J.-W. Choi, M. Zloh and S. Shaunak, Nat. Protoc., 2006, 1, 2241-2252.

369 J. M. Thornton, J. Mol. Biol., 1981, 151, 261-287.

370 R. Bhattacharyya, D. Pal and P. Chakrabarti, Protein Eng., 2004, 17, 795-808.

371 S. Shaunak, A. Godwin, J.-W. Choi, S. Balan, E. Pedone, D. Vijayarangam, S. Heidelberger, I. Teo, M. Zloh and S. Brocchini, Nat. Chem. Biol., 2006, 2, 312-313.

372 S. Balan, J.-W. Choi, A. Godwin, I. Teo, C. M. Laborde, S. Heidelberger, M. Zloh, S. Shaunak and S. Brocchini, Bioconjugate Chem., 2007, 18, 61-76.

373 S. Brocchini, A. Godwin, S. Balan, J.-W. Choi, M. Zloh and S. Shaunak, Adv. Drug Delivery Rev., 2008, 60, 3-12.

374 H. Khalili, A. Godwin, J.-W. Choi, R. Lever and S. Brocchini, Bioconjugate Chem., 2012, 23, 2262-2277.

375 A. Lewis, Y. Tang, S. Brocchini, J.-W. Choi and A. Godwin, Bioconjugate Chem., 2008, 19, 2144-2155.

376 L. Castañeda, Z. V. F. Wright, C. Marculescu, T. M. Tran, V. Chudasama, A. Maruani, E. A. Hull, J. P. M. Nunes, 
R. J. Fitzmaurice, M. E. B. Smith, L. H. Jones, S. Caddick and J. R. Baker, Tetrahedron Lett., 2013, 54, 3493-3495.

377 M. W. Jones, R. A. Strickland, F. F. Schumacher, S. Caddick, J. R. Baker, M. I. Gibson and D. M. Haddleton, J. Am. Chem. Soc., 2012, 134, 1847-1852.

378 F. F. Schumacher, V. A. Sanchania, B. Tolner, Z. V. F. Wright, C. P. Ryan, M. E. B. Smith, J. M. Ward, S. Caddick, C. W. M. Kay, G. Aeppli, K. A. Chester and J. R. Baker, Sci. Rep., 2013, 3, 1-8.

379 M. E. B. Smith, J. Baker, F. F. Schumacher, S. Caddick, V. Chudasama and A. Maruani, WO2013132268, 2013, https://patentscope.wipo.int/search/en/detail.jsf?docId= WO2013132268.

380 F. F. Schumacher, M. Nobles, C. P. Ryan, M. E. Smith, A. Tinker, S. Caddick and J. R. Baker, Bioconjugate Chem., 2011, 22, 132-136.

381 M. W. Jones, R. A. Strickland, F. F. Schumacher, S. Caddick, J. R. Baker, M. I. Gibson and D. M. Haddleton, Chem. Commun., 2012, 48, 4064-4066.

382 J. Collins, J. Tanaka, P. Wilson, K. Kempe, T. P. Davis, M. P. McIntosh, M. R. Whittaker and D. M. Haddleton, Bioconjugate Chem., 2015, 26, 633-638.

383 V. Chudasama, M. E. B. Smith, F. F. Schumacher, D. Papaioannou, G. Waksman, J. R. Baker and S. Caddick, Chem. Commun., 2011, 47, 8781-8783.

384 A. Maruani, M. E. B. Smith, E. Miranda, K. A. Chester, V. Chudasama and S. Caddick, Nat. Commun., 2015, 6, 6645.

385 M. J. Tucker, J. R. Courter, J. Chen, O. Atasoylu, A. B. Smith and R. M. Hochstrasser, Angew. Chem., Int. Ed., 2010, 49, 3612-3616.

386 S. P. Brown and A. B. Smith, J. Am. Chem. Soc., 2015, 137, 4034-4037.

387 P. Wilson, A. Anastasaki, M. R. Owen, K. Kempe, D. M. Haddleton, S. K. Mann, A. P. R. Johnston, J. F. Quinn, M. R. Whittaker, P. J. Hogg and T. P. Davis, J. Am. Chem. Soc., 2015, 137, 4215-4222.

388 C. J. Chapman, K. J. Wadsworth and C. G. Frost, J. Organomet. Chem., 2003, 680, 206-211.

389 C. J. Chapman, A. Matsuno, C. G. Frost and M. C. Willis, Chem. Commun., 2007, 3903-3905.

390 C. J. Chapman, J. D. Hargrave, G. Bish and C. G. Frost, Tetrahedron, 2008, 64, 9528-9539.

391 L. Navarre, R. Martinez, J.-P. Genet and S. Darses, J. Am. Chem. Soc., 2008, 130, 6159-6169.

392 G. J. L. Bernardes, J. M. Chalker, J. C. Errey and B. G. Davis, J. Am. Chem. Soc., 2008, 130, 5052-5053.

393 J. M. Chalker, L. Lercher, N. R. Rose, C. J. Schofield and B. G. Davis, Angew. Chem., Int. Ed., 2012, 124, 1871-1875.

394 P. N. Collier, A. D. Campbell, I. Patel and R. J. K. Taylor, Tetrahedron Lett., 2000, 41, 7115-7119.

395 J. M. Chalker, S. B. Gunnoo, O. Boutureira, S. C. Gerstberger, M. Fernández-González, G. J. L. Bernardes, L. Griffin, H. Hailu, C. J. Schofield and B. G. Davis, Chem. Sci., 2011, 2, 1666-1676.

396 I. Photaki, J. Am. Chem. Soc., 1963, 85, 1123-1126.

397 D. H. Strumeyer, W. N. White and D. E. Koshland, Proc. Natl. Acad. Sci. U. S. A., 1963, 50, 931-935.
398 H. Weiner, W. N. White, D. G. Hoare and D. E. Koshland, J. Am. Chem. Soc., 1966, 88, 3851-3859.

399 D. H. Rich, J. Tam, P. Mathiaparanam, J. A. Grant and C. Mabuni, J. Chem. Soc., Chem. Commun., 1974, 897-898.

400 J. Chalker, G. Bernardes, Y. Lin and B. Davis, Chem. - Asian J., 2009, 4, 630-640.

401 T. J. Holmes and R. G. Lawton, J. Am. Chem. Soc., 1977, 99, 1984-1986.

402 F. C. Rowan, M. Richards, R. A. Bibby, A. Thompson, R. Bayliss and J. Blagg, ACS Chem. Biol., 2013, 8, 2184-2191.

403 R. I. Nathani, P. Moody, V. Chudasama, M. E. B. Smith, R. J. Fitzmaurice and S. Caddick, Chem. Sci., 2013, 4, 3455-3458.

404 K. Hashimoto, M. Sakai, T. Okuno and H. Shirahama, Chem. Commun., 1996, 1139-1140.

405 N. M. Okeley, Y. Zhu and W. A. van der Donk, Org. Lett., 2000, 2, 3603-3606.

406 F. P. Seebeck and J. W. Szostak, J. Am. Chem. Soc., 2006, 128, 7150-7151.

407 J. Wang, S. M. Schiller and P. G. Schultz, Angew. Chem., Int. Ed., 2007, 119, 6973-6975.

408 J. Guo, J. Wang, J. S. Lee and P. G. Schultz, Angew. Chem., Int. Ed., 2008, 120, 6499-6501.

409 Y. O. You, M. R. Levengood, L. A. F. Ihnken, A. K. Knowlton and W. A. van der Donk, ACS Chem. Biol., 2009, 4, 379-385.

410 Y. A. Lin, O. Boutureira and L. Lercher, J. Am. Chem. Soc., 2013, 135, 12156-12159.

411 H. Lindley, Nature, 1956, 178, 647-648.

412 M. A. Raftery and R. D. Cole, J. Biol. Chem., 1966, 241, 3457-3461.

413 M. D. Simon, F. Chu, L. R. Racki, C. C. de la Cruz, A. L. Burlingame, B. Panning, G. J. Narlikar and K. M. Shokat, Cell, 2007, 128, 1003-1012.

414 A. M. Spokoyny, Y. Zou, J. J. Ling, H. Yu, Y.-S. Lin and B. L. Pentelute, J. Am. Chem. Soc., 2013, 135, 5946-5949.

415 C. Zhang, A. M. Spokoyny, Y. Zou, M. D. Simon and B. L. Pentelute, Angew. Chem., Int. Ed., 2013, 52, 1-6.

416 N. Toda, S. Asano and C. F. Barbas III, Angew. Chem., Int. Ed., 2013, 52, 12592-12596.

417 H.-Y. Shiu, T.-C. Chan, C.-M. Ho, Y. Liu, M.-K. Wong and C.-M. Che, Chem. - Eur. J., 2009, 15, 3839-3850.

418 C. Heinis, T. Rutherford, S. Freund and G. Winter, Nat. Chem. Biol., 2009, 5, 502-507.

419 S. Chen, J. Touati and C. Heinis, Chem. Commun., 2014, 50, 5267-5269.

420 A. O.-Y. Chan, J. L.-L. Tsai, V. K.-Y. Lo, G.-L. Li, M.-K. Wong and C.-M. Che, Chem. Commun., 2013, 49, 1428-1430.

421 P. D. Halphen and T. C. Owen, J. Org. Chem., 1973, 38, 3507-3510.

422 W. E. Truce and G. J. W. Tichenor, J. Org. Chem., 1972, 37, 2391-2396.

423 O. De Lucchi, V. Lucchini, C. Marchioro, G. Valle and G. Modena, J. Org. Chem., 1986, 51, 1457-1466.

424 M. Journet, A. Rouillard, D. Cai and R. D. Larsen, J. Org. Chem., 1997, 62, 8630-8631.

425 O. Arjona, R. Medel, J. Rojas, A. M. Costa and J. Vilarrasa, Tetrahedron Lett., 2003, 44, 6369-6373. 
426 B. A. Trofimov, A. G. Mal'kina, O. A. Shemyakina, V. V. Nosyreva, A. P. Borisova, S. S. Khutsishvili and L. B. Krivdin, Synthesis, 2009, 3136-3142.

427 H.-Y. Shiu, H.-C. Chong, Y.-C. Leung, M.-K. Wong and C.-M. Che, Chem. - Eur. J., 2010, 16, 3308-3313.

428 O. Koniev, G. Leriche, M. Nothisen, J.-S. Remy, J.-M. Strub, C. Schaeffer-Reiss, A. Van Dorsselaer, R. Baati and A. Wagner, Bioconjugate Chem., 2014, 25, 202-206.

429 S. Kolodych, O. Koniev, Z. Baatarkhuu, J.-Y. Bonnefoy, F. Debaene, S. Cianférani, A. Van Dorsselaer and A. Wagner, Bioconjugate Chem., 2015, 26, 197-200.

430 V. Hong, A. A. Kislukhin and M. G. Finn, J. Am. Chem. Soc., 2009, 131, 9986-9994.

431 S. Dziadek, S. Jacques and D. R. Bundle, Chemistry, 2008, 14, 5908-5917.

432 M. W. Jones, G. Mantovani, S. M. Ryan, X. Wang, D. J. Brayden and D. M. Haddleton, Chem. Commun., 2009, 5272-5274.

433 C. Haase and O. Seitz, Justus Liebigs Ann. Chem., 2009, 2096-2101.

434 F. K. Hansen, K. Ha, E. Todadze, A. Lillicotch, A. Frey and A. R. Katritzky, Org. Biomol. Chem., 2011, 9, 7162-7167.

435 K. Ha, M. Chahar, J.-C. M. Monbaliu, E. Todadze, F. K. Hansen, A. A. Oliferenko, C. E. Ocampo, D. Leino, A. Lillicotch, C. V. Stevens and A. R. Katritzky, J. Org. Chem., 2012, 77, 2637-2648.

436 O. Bol'shakov, J. Kovacs, M. Chahar, K. Ha, L. Khelashvili and A. R. Katritzky, J. Pept. Sci., 2012, 18, 704-709.

437 S. S. Panda, C. El-Nachef, K. Bajaj, A. O. Al-Youbi, A. Oliferenko and A. R. Katritzky, Chem. Biol. Drug Des., 2012, 80, 821-827.

438 J.-C. M. Monbaliu, G. Dive, C. V. Stevens and A. R. Katritzky, J. Chem. Theory Comput., 2013, 9, 927-934.

439 K. Gevaert, P. Van Damme, L. Martens and J. Vandekerckhove, Anal. Biochem., 2005, 345, 18-29.

440 D. E. Koshland, Y. D. Karkhanis and H. G. Latham, J. Am. Chem. Soc., 1964, 86, 1448-1450.

441 W. E. Savige and A. Fontana, Methods Enzymol., 1977, 47, 442-453.

442 J. E. Bell, F. J. Castellino, I. P. Trayer and R. L. Hill, J. Biol. Chem., 1975, 250, 7579-7585.

443 H. R. Horton and D. E. Koshland, J. Am. Chem. Soc., 1965, 87, 1126-1132.

444 M. Strohalm, J. Šantrůček, R. Hynek and M. Kodíček, Biochem. Biophys. Res. Commun., 2004, 323, 1134-1138.

445 H. J. Teuber, O. Glosauer and U. Hochmuth, Eur. J. Inorg. Chem., 1964, 557-562.

446 A. Foettinger, M. Melmer, A. Leitner and W. Lindner, Bioconjugate Chem., 2007, 18, 1678-1683.

447 M. Källberg, H. Wang, S. Wang, J. Peng, Z. Wang, H. Lu and J. Xu, Nat. Protoc., 2012, 7, 1511-1522.

448 A. Foettinger, A. Leitner and W. Lindner, J. Mass Spectrom., 2006, 41, 623-632.

449 A. Foettinger, A. Leitner and W. Lindner, J. Proteome Res., 2007, 6, 3827-3834.

450 J. Antos and M. Francis, J. Am. Chem. Soc., 2004, 126, 10256-10257.
451 T. Ye and M. A. McKervey, Macromolecules, 1994, 94, 1091-1160.

452 J. M. Antos, J. M. McFarland, A. T. Iavarone and M. B. Francis, J. Am. Chem. Soc., 2009, 131, 6301-6308.

453 J. M. Antos and M. B. Francis, J. Am. Chem. Soc., 2004, 126, 10256-10257.

454 B. V. Popp and Z. T. Ball, J. Am. Chem. Soc., 2010, 132, 6660-6662.

455 D. N. Woolfson, Adv. Protein Chem., 2005, 70, 79-112.

456 Z. Chen, B. V. Popp, C. L. Bovet and Z. T. Ball, ACS Chem. Biol., 2011, 6, 920-925.

457 J. L. Howland, The Encyclopedia of Molecular Biology, Biochemical Education, 1995.

458 S. Li and M. Hong, J. Am. Chem. Soc., 2011, 133, 1534-1544. 459 X. Li, H. Ma, S. Dong, X. Duan and S. Liang, Talanta, 2004, 62, 367-371.

460 X. Li, H. Ma, L. Nie, M. Sun and S. Xiong, Anal. Chim. Acta, 2004, 515, 255-260.

461 R. E. Parker and N. S. Isaacs, Chem. Rev., 1959, 59, 737-799.

462 Y. Takaoka, H. Tsutsumi, N. Kasagi, E. Nakata and I. Hamachi, J. Am. Chem. Soc., 2006, 128, 3273-3280.

463 H. Wakabayashi, M. Miyagawa, Y. Koshi, Y. Takaoka, S. Tsukiji and I. Hamachi, Chem. - Asian J., 2008, 3, 1134-1139.

464 N. Miyaura and A. Suzuki, Macromolecules, 1995, 95, 2457-2483.

465 M. Meldal and C. W. Tornøe, Macromolecules, 2008, 108, 2952-3015.

466 J. Z. Hearon, J. Natl. Cancer Inst., 1948, 9, 1-11.

467 X. Wang, J. Jia, Z. Huang, M. Zhou and H. Fei, Chem. - Eur. J., 2011, 17, 8028-8032.

468 D.-L. Ma, W.-L. Wong, W.-H. Chung, F.-Y. Chan, P.-K. So, T.-S. Lai, Z.-Y. Zhou, Y.-C. Leung and K.-Y. Wong, Angew. Chem., Int. Ed., 2008, 47, 3735-3739.

469 K. Uchida and E. R. Stadtman, Proc. Natl. Acad. Sci. U. S. A., 1992, 89, 4544-4548.

470 R. Zamora, M. Alaiz and F. J. Hidalgo, Chem. Res. Toxicol., 1999, 12, 654-660.

471 F. J. Hidalgo and R. Zamora, J. Biol. Chem., 1993, 268, 16190-16197.

472 R. Chand Bollineni, M. Fedorova and R. Hoffmann, Analyst, 2013, 138, 5081-5088.

473 D. Wylie, M. Voloch, S. Lee, Y. H. Liu, S. Cannon Carlson, C. Cutler and B. Pramanik, Pharm. Res., 2001, 18, 1354-1360.

474 T. Nishino, V. Massey and C. H. Williams, J. Biol. Chem., 1980, 255, 3610-3616.

475 E. B. Williams, S. Krishnaswamy and K. G. Mann, J. Biol. Chem., 1989, 264, 7536-7545.

476 R. G. Fruchter and A. M. Crestfield, J. Biol. Chem., 1967, 242, 5807-5812.

477 M. C. Lin, W. H. Stein and S. Moore, J. Biol. Chem., 1968, 243, 6167-6170.

478 T. T. Inagami and H. H. Hatano, J. Biol. Chem., 1969, 244, 1176-1182.

479 T. Y. Liu, J. Biol. Chem., 1967, 242, 4029-4032.

480 H. Pauly, Hoppe-Seyler's Z. Physiol. Chem., 1905, 159-160. 
481 H. Pauly, Hoppe-Seyler's Z. Physiol. Chem., 1915, 94, 284-290.

482 F. Zaheer and B. H. Nicholson, Biochim. Biophys. Acta, Protein Struct., 1971, 251, 38-43.

483 P. Dominici, B. Tancini and C. Borri Voltattorni, J. Biol. Chem., 1985, 260, 10583-10589.

484 F. Marks, U. Klingmüller and K. Müller-Decker, Cellular signal processing: an introduction to the molecular mechanisms of signal transduction, lavoisier.eu, 2009.

485 M. A. Lemmon and J. Schlessinger, Cell, 2010, 141, 1117-1134.

486 Y. Koshi, E. Nakata, M. Miyagawa, S. Tsukiji, T. Ogawa and I. Hamachi, J. Am. Chem. Soc., 2008, 130, 245-251.

487 S. Håkansson, J. Viljanen and K. S. Broo, Biochemistry, 2003, 42, 10260-10268.

488 T. Shirai, Y. Matsui, C. Shionyu-Mitsuyama, T. Yamane, H. Kamiya, C. Ishii, T. Ogawa and K. Muramoto, J. Mol. Biol., 2002, 321, 879-889.

489 J. F. Riordan, M. Sokolovsky and B. L. Vallee, J. Am. Chem. Soc., 1966, 88, 4104-4105.

490 K. Jiao, S. Mandapati, P. L. Skipper, S. R. Tannenbaum and J. S. Wishnok, Anal. Biochem., 2001, 293, 43-52.

491 H. Neurath and R. A. Kenner, Biochemistry, 1971, 10, 551-557.

492 G. Nikov, V. Bhat, J. S. Wishnok and S. R. Tannenbaum, Anal. Biochem., 2003, 320, 214-222.

493 M. Kai, J. Ishida and Y. Ohkura, J. Chromatogr. B, Biomed. Appl., 1988, 430, 271-278.

494 J. Ishida, M. Kai and Y. Ohkura, J. Chromatogr. A, 1986, 356, 171-177.

495 B. M. Trost and F. D. Toste, J. Am. Chem. Soc., 1998, 120, 9074-9075.

496 B. M. Trost and F. D. Toste, J. Am. Chem. Soc., 1998, 120, 815-816.

497 S. Tilley and M. Francis, J. Am. Chem. Soc., 2006, 128, 1080-1081.

498 K. C. Brown, S.-H. Yang and T. Kodadek, Biochemistry, 1995, 34, 4733-4739.

499 K. C. Brown, Z. Yu, A. L. Burlingame and C. S. Craik, Biochemistry, 1998, 37, 4397-4406.

500 D. A. Fancy, K. Melcher, S. A. Johnston and T. Kodadek, Chem. Biol., 1996, 3, 551-559.

501 D. A. Fancy and T. Kodadek, Tetrahedron, 1997, 53, 11953-11960.

502 G. Gill, A. A. Richter-Rusli, M. Ghosh, C. J. Burrows and S. E. Rokita, Chem. Res. Toxicol., 1997, 10, 302-309.

503 K. Kim, D. A. Fancy, D. Carney and T. Kodadek, J. Am. Chem. Soc., 1999, 121, 11896-11897.

504 D. A. Fancy and T. Kodadek, Proc. Natl. Acad. Sci. U. S. A., 1999, 96, 6020-6024.

505 D. A. Fancy, C. Denison, K. Kim, Y. Xie, T. Holdeman, F. Amini and T. Kodadek, Chem. Biol., 2000, 7, 697-708.

506 M. D. Person, K. C. Brown, S. Mahrus, C. S. Craik and A. L. Burlingame, Protein Sci., 2009, 10, 1549-1562.

507 T. Kodadek, I. Duroux-Richard and J. Bonnafous, Trends Pharmacol. Sci., 2005, 26, 210-217.
508 J. Antos and M. Francis, Curr. Opin. Chem. Biol., 2006, 10, 253-262.

509 K. L. Seim, A. C. Obermeyer and M. B. Francis, J. Am. Chem. Soc., 2011, 133, 16970-16976.

510 I. Duroux-Richard, P. Vassault, G. Subra, J.-F. Guichou, E. Richard, B. Mouillac, C. Barberis, J. Marie and J.-C. Bonnafous, Chem. Biol., 2005, 12, 15-24.

511 C. Denison, J. Proteome Res., 2004, 3, 417-425.

512 S. Meunier, E. Strable and M. G. Finn, Chem. Biol., 2004, 11, 319-326.

513 H. G. Higgins and K. J. Harrington, Arch. Biochem. Biophys,, 1959, 85, 409-425.

514 A. Roglans, A. Pla-Quintana and M. Moreno-Mañas, Chem. Rev., 2006, 106, 4622-4643.

515 J. M. Hooker, E. W. Kovacs and M. B. Francis, J. Am. Chem. Soc., 2004, 126, 3718-3719.

516 J. M. Hooker, A. Datta, M. Botta, K. N. Raymond and M. B. Francis, Nano Lett., 2007, 7, 2207-2210.

517 T. L. Schlick, Z. Ding, E. W. Kovacs and M. B. Francis, J. Am. Chem. Soc., 2005, 127, 3718-3723.

518 M. W. Jones, G. Mantovani, C. A. Blindauer, S. M. Ryan, X. Wang, D. J. Brayden and D. M. Haddleton, J. Am. Chem. Soc., 2012, 134, 7406-7413.

519 J. Gavrilyuk, H. Ban, M. Nagano, W. Hakamata and C. F. Barbas, Bioconjugate Chem., 2012, 23, 2321-2328.

520 H. Fraenkel-Conrat and H. S. Olcott, J. Biol. Chem., 1948, 174, 827-843.

521 N. S. Joshi, L. R. Whitaker and M. B. Francis, J. Am. Chem. Soc., 2004, 126, 15942-15943.

522 D. Romanini and M. Francis, Bioconjugate Chem., 2008, 19, 153-157.

523 J. M. McFarland, N. S. Joshi and M. B. Francis, J. Am. Chem. Soc., 2008, 130, 7639-7644.

524 H.-M. Guo, M. Minakawa and F. Tanaka, Chem. Rev., 2008, 73, 3964-3966.

525 M. Minakawa, H.-M. Guo and F. Tanaka, J. Org. Chem., 2008, 73, 8669-8672.

526 S. H. Schroeter, J. Org. Chem., 1969, 34, 4012-4015.

527 H. Mitchell and Y. Leblanc, J. Org. Chem., 1994, 59, 682-687.

528 Y. Leblanc and N. Boudreault, J. Org. Chem., 1995, 60, 4268-4271.

529 J. S. Yadav, B. V. S. Reddy, G. M. Kumar and C. Madan, Synlett, 2001, 1781-1783.

530 J. S. Yadav, B. V. S. Reddy, G. Veerendhar, R. S. Rao and K. Nagaiah, Chem. Lett., 2002, 318-319.

531 S. Bombek, R. Lenaršič and M. Kočevar, Chem. Commun., 2002, 1494-1495.

532 W. J. Kinart and C. M. Kinart, J. Organomet. Chem., 2003, 665, 233-236.

533 G. L. Chee, Synth. Commun., 2006, 36, 2151-2156.

534 S. Brandes, M. Bella, A. Kjærsgaard and K. A. Jørgensen, Angew. Chem., Int. Ed., 2006, 45, 1147-1151.

535 G. Desimoni, G. Faita, P. P. Righetti, A. Sfulcini and D. Tsyganov, Tetrahedron, 1994, 50, 1821-1832.

536 H. Ban, J. Gavrilyuk and I. C. F. Barbas, J. Am. Chem. Soc., 2010, 132, 1523-1525. 
537 H. Ban, M. Nagano, J. Gavrilyuk, W. Hakamata, T. Inokuma and C. F. Barbas, Bioconjugate Chem., 2013, 24, 520-532.

538 O. Y. Wong, P. I. Pradeepkumar and S. K. Silverman, Biochemistry, 2011, 50, 4741-4749.

539 C. L. Borders, J. A. Broadwater, P. A. Bekeny, J. E. Salmon, A. S. Lee, A. M. Eldridge and V. B. Pett, Protein Sci., 1994, 3, 541-548.

540 L. Shimoni and J. P. Glusker, Protein Sci., 1995, 4, 65-74.

541 R. M. C. Dawson, Data for Biochemical Research, Oxford University Press, USA, 3rd edn, 1989.

542 B. H. McMahon, M. Fabian, F. Tomson, T. P. Causgrove, J. A. Bailey, F. N. Rein, R. B. Dyer, G. Palmer, R. B. Gennis and W. H. Woodruff, Biochim. Biophys. Acta, 2004, 1655, 321-331.

543 Y. Xiao, M. S. Hutson, M. Belenky, J. Herzfeld and M. S. Braiman, Biochemistry, 2004, 43, 12809-12818.

544 A. Leitner and W. Lindner, Anal. Chem., 2005, 77, 4481-4488.

545 K. Takahashi, J. Biol. Chem., 1968, 243, 6171-6179.

546 N. A. R. R. Manohar, Biochem. J., 1984, 224, 703-707.

547 C. L. Borders, J. E. Saunders, D. M. Blech and I. Fridovich, Biochem. J., 1985, 230, 771-776.

548 G. Bailin, Biochem. Int., 1985, 11, 161-170.

549 K. Varalakshmi, H. S. Savithri and N. A. Rao, Biochem. J., 1986, 236, 295-298.

550 H. M. Eun, Biochem. Int., 1988, 6171-6179.

551 R. B. Yamasaki, A. Vega and R. E. Feeney, Anal. Biochem., 1980, 109, 32-40.

552 R. B. Yamasaki, D. A. Shimer and R. E. Feeney, Anal. Biochem., 1981, 111, 220-226.

553 J. Strévey, V. Vachon, B. Beaumier, S. Giroux and R. Béliveau, Biochim. Biophys. Acta, 1992, 1106, 110-116.

554 E. B. Mukouyama, T. Hirose and H. Suzuki, J. Biochem., 1998, 123, 1097-1103.

555 T. T. Ngo, C. F. Yam, H. M. Lenhoff and J. Ivy, J. Biol. Chem., 1981, 256, 11313-11318.

556 S.-T. Cheung and M. L. Fonda, Biochem. Biophys. Res. Commun., 1979, 90, 940-947.

557 J. A. Yankeelov Jr, Biochemistry, 1970, 9, 2433-2439.

558 J. A. Yankeelov Jr, Methods Enzymol., 1972, 25, 566-579.

559 K. Toi, E. Bynum, E. Norris and H. A. Itano, J. Biol. Chem., 1967, 242, 1036-1043.

560 J. F. Riordan, Biochemistry, 1973, 12, 3915-3923.

561 L. Patthy and E. L. Smith, J. Biol. Chem., 1975, 250, 557-564.

562 A. Foettinger, A. Leitner and W. Lindner, J. Chromatogr. A, 2005, 1079, 187-196.

563 S. M. Politz, H. F. Noller and P. D. McWhirter, Biochemistry, 1981, 20, 372-378.

564 M. A. Gauthier and H.-A. Klok, Biomacromolecules, 2011, 12, 482-493.

565 K. Takahashi, J. Biochem., 1976, 80, 1173-1176.

566 M. Pischetsrieder, J. Agric. Food Chem., 1996, 44, 2081-2085.

567 D. G. Hoare and D. E. Koshland Jr, J. Am. Chem. Soc., 1966, 88, 2057-2058.

568 D. G. Hoare and D. E. Koshland, J. Biol. Chem., 1967, 242, 2447-2453.
569 H. G. Khorana, Chem. Rev., 1953, 53, 145-166.

570 R. Renthal, M. Cothran, N. Dawson and G. J. Harris, Biochim. Biophys. Acta, 1987, 897, 384-394.

571 A. Das and L. G. Ljungdahl, J. Bacteriol., 1997, 179, 3746-3755.

572 G. M. Cook, S. Keis, H. W. Morgan, C. von Ballmoos, U. Matthey, G. Kaim and P. Dimroth, J. Bacteriol., 2003, 185, 4442-4449.

573 J. C. Sheehan and J. J. Hlavka, J. Org. Chem., 1956, 21, 439-441.

574 J. C. Sheehan and J. J. Hlavka, J. Am. Chem. Soc., 1957, 79, 4528-4529.

575 K. L. Carraway and D. E. Koshland Jr, Methods Enzymol., 1972, 25, 616-623.

576 T. L. Goodfriend, L. Levine and G. D. Fasman, Science, 1964, 144, 1344-1346.

577 N. Xia, Y. Xing, G. Wang, Q. Feng, Q. Chen, H. Feng, X. Sun and L. Liu, Int. J. Electrochem. Sci., 2013, 8, 2459-2467.

578 M. A. Gilles, A. Q. Hudson and C. L. Borders, Anal. Biochem., 1990, 184, 244-248.

579 E. Valeur and M. Bradley, Chem. Soc. Rev., 2009, 38, 606-631.

580 R. B. Woodward, R. A. Olofson and H. Mayer, J. Am. Chem. Soc., 1961, 83, 1010-1012.

581 P. Bodlaender, G. Feinstein and E. Shaw, Biochemistry, 1969, 8, 4941-4949.

582 B. F. Erlanger, S. M. Vratsanos, N. Wassermann and A. G. Cooper, J. Biol. Chem., 1965, 240, PC3447-PC3448.

583 E. Gross and J. L. Morell, J. Biol. Chem., 1966, 241, 3638-3639.

584 K. Takahashi, W. H. Stein and S. Moore, J. Biol. Chem., 1967, 242, 4682-4690.

585 W. Vogt, Free Radical Biol. Med., 1995, 18, 93-105.

586 A. N. Glazer, Annu. Rev. Biochem., 1970, 39, 101-130.

587 K. Lindorff-Larsen and J. R. Winther, Anal. Biochem., 2000, 286, 308-310.

588 J. R. Kramer and T. J. Deming, Chem. Commun., 2013, 49, 5144-5146.

589 G. Toennies, J. Biol. Chem., 1940, 132, 455-456.

590 G. Toennies and J. J. Kolb, J. Am. Chem. Soc., 1945, 67, 849-851.

591 T.-L. Ho, Macromolecules, 1975, 75, 1-20.

592 E. Reid, Nature, 1951, 1, 955.

593 O. Kinstler, G. Molineux, M. Treuheit, D. Ladd and C. Gegg, Adv. Drug Delivery Rev., 2002, 54, 477-485.

594 G. Gaudriault and J. P. Vincent, Peptides, 1992, 13, 1187-1192.

595 I. Sélo, L. Négroni, C. Créminon, J. Grassi and J. M. Wal, J. Immunol. Methods, 1996, 199, 127-138.

596 M. Yamaguchi, T. Nakazawa, H. Kuyama, T. Obama, E. Ando, T.-A. Okamura, N. Ueyama and S. Norioka, Anal. Chem., 2005, 77, 645-651.

597 G. Xu and S. R. Jaffrey, Cold. Spring. Harb. Protoc., 2010, 2010, 1-15 (pdb.prot5528).

598 D. Bertaccini, S. Vaca, C. Carapito, F. Arsène-Ploetze, A. Van Dorsselaer and C. Schaeffer-Reiss, J. Proteome Res., 2013, 12, 3063-3070. 
599 T. M. Rana and C. F. Meares, Bioconjugate Chem., 1990, 1, 357-362.

600 H. B. F. Dixon, J. Protein Chem., 1984, 3, 99-108.

601 J. M. Gilmore, R. A. Scheck, A. P. Esser-Kahn, N. S. Joshi and M. B. Francis, Angew. Chem., Int. Ed., 2006, 45, 5307-5311.

602 W.-K. Chan, C.-M. Ho, M.-K. Wong and C.-M. Che, J. Am. Chem. Soc., 2006, 128, 14796-14797.

603 A. O.-Y. Chan, C.-M. Ho, H.-C. Chong, Y.-C. Leung, J.-S. Huang, M.-K. Wong and C.-M. Che, J. Am. Chem. Soc., 2012, 134, 2589-2598.

604 H. Dixon and L. R. Weitkamp, Biochem. J., 1962, 34, 462-468.

605 W. P. Jencks, J. Am. Chem. Soc., 1959, 81, 475-481.

606 J. Kalia and R. T. Raines, Angew. Chem., Int. Ed., 2008, 47, 7523-7526.

607 D. E. Metzler and E. E. Snell, J. Am. Chem. Soc., 1952, 74, 979-983.

608 C. Cennamo, Naturwissenschaften, 1954, 41, 39.

609 C. Cennamo, B. Carafoli and E. P. Bonetti, J. Am. Chem. Soc., 1956, 78, 3523-3527.

610 H. Dixon, Biochem. J., 1964, 92, 661-666.

611 H. Dixon and V. Moret, Biochem. J., 1965, 94, 463-469.

612 H. Mix and F. W. Wilcke, Hoppe-Seyler's Z. Physiol. Chem., 1960, 318, 148-158.

613 S. Van Heyningen and H. B. Dixon, Biochem. J., 1969, 114, 70-71.

614 H. B. Dixon and R. Fields, Methods Enzymol., 1972, 25, 409-419.

615 L. S. Witus and M. Francis, Curr. Protoc. Chem. Biol., 2010, 2, 125-134.

616 R. A. Scheck, M. T. Dedeo, A. T. Iavarone and M. B. Francis, J. Am. Chem. Soc., 2008, 130, 11762-11770.

617 L. S. Witus, T. Moore, B. W. Thuronyi, A. P. Esser-Kahn, R. A. Scheck, A. T. Iavarone and M. B. Francis, J. Am. Chem. Soc., 2010, 132, 16812-16817.

618 R. A. Scheck and M. B. Francis, ACS Chem. Biol., 2007, 2, 247-251.

619 L. S. Witus, C. Netirojjanakul, K. S. Palla, E. M. Muehl, C.-H. Weng, A. T. Iavarone and M. B. Francis, J. Am. Chem. Soc., 2013, 135, 17223-17229.

620 K. S. Palla, L. S. Witus, K. J. Mackenzie, C. Netirojjanakul and M. B. Francis, J. Am. Chem. Soc., 2015, 137, 1123-1129.

621 M. Zhang, X. Zhang, J. Li, Q. Guo and Q. Xiao, Chin. J. Chem., 2011, 29, 1715-1720.

622 M. Dettin, N. Muncan, A. Bugatti, F. Grezzo, R. Danesin and M. Rusnati, Bioconjugate Chem., 2011, 22, 1753-1757.

623 A. P. Crochet, M. M. Kabir, M. B. Francis and C. D. Paavola, Biosens. Bioelectron., 2010, 26, 55-61.

624 W. Gao, W. Liu, J. A. Mackay, M. R. Zalutsky, E. J. Toone and A. Chilkoti, Proc. Natl. Acad. Sci. U. S. A., 2009, 106, 15231-15236.

625 Z. M. Carrico, M. E. Farkas, Y. Zhou, S. C. Hsiao, J. D. Marks, H. Chokhawala, D. S. Clark and M. B. Francis, ACS Nano, 2012, 6, 6675-6680.

626 K. K. Palaniappan, R. M. Ramirez, V. S. Bajaj, D. E. Wemmer, A. Pines and M. B. Francis, Angew. Chem., Int. Ed., 2013, 125, 4949-4953.
627 K. Sonomura, H. Kuyama, E.-I. Matsuo, S. Tsunasawa and O. Nishimura, Bioorg. Med. Chem. Lett., 2009, 19, 6544-6547.

628 K. Sonomura, H. Kuyama, E.-I. Matsuo, S. Tsunasawa, S. Futaki and O. Nishimura, Anal. Biochem., 2011, 410, 214-223.

629 M.-J. Han, D.-C. Xiong and X.-S. Ye, Chem. Commun., 2012, 48, 11079-11081.

630 T. Sasaki, K. Kodama, H. Suzuki, S. Fukuzawa and K. Tachibana, Bioorg. Med. Chem. Lett., 2008, 18, 4550-4553.

631 P. Agarwal, J. van der Weijden, E. M. Sletten, D. Rabuka and C. R. Bertozzi, Proc. Natl. Acad. Sci. U. S. A., 2013, 110, 46-51.

632 J. I. MacDonald, H. K. Munch, T. Moore and M. B. Francis, Nat. Chem. Biol., 2015, 11, 326-331.

633 J. P. Waller and H. B. Dixon, Biochem. J., 1960, 75, 320-328.

634 M. Brenner, J. P. Zimmermann, J. Wehrmüller, P. Quitt and I. Photaki, Experientia, 1955, 11, 397-399.

635 C. F. C. Liu and J. P. J. Tam, Proc. Natl. Acad. Sci. U. S. A., 1994, 91, 6584-6588.

636 C.-F. Liu and J. P. Tam, J. Am. Chem. Soc., 1994, 116, 4149-4153.

637 J. P. Tam, C. Rao, C. F. Liu and J. Shao, Int. J. Pept. Protein Res., 1995, 45, 209-216.

638 Z. Miao and J. P. Tam, J. Am. Chem. Soc., 2000, 122, 4253-4260.

639 J. P. Tam, Q. Yu and J. L. Yang, J. Am. Chem. Soc., 2001, 123, 2487-2494.

640 R. Fields and H. Dixon, Biochem. J., 1968, 106, 883-887.

641 K. F. Geoghegan and J. G. Stroh, Bioconjugate Chem., 1992, 3, 138-146.

642 B. H. Nicolet and L. A. Shinn, J. Am. Chem. Soc., 1939, 61, 1615.

643 L. Zhang and J. P. Tam, Anal. Biochem., 1996, 233, 87-93.

644 H. F. Gaertner, K. Rose, R. Cotton, D. Timms, R. Camble and R. E. Offord, Bioconjugate Chem., 1992, 3, 262-268.

645 J. Chen, W. Zeng, R. Offord and K. Rose, Bioconjugate Chem., 2003, 14, 614-618.

646 K. F. Geoghegan, M. J. Emery, W. H. Martin, A. S. McColl and G. O. Daumy, Bioconjugate Chem., 1993, 4, 537-544.

647 S. D. Mikolajczyk, D. L. Meyer, J. J. Starling, K. L. Law, K. Rose, B. Dufour and R. E. Offord, Bioconjugate Chem., 1994, 5, 636-646.

648 K. Rose, W. Zeng, P.-O. Regamey, I. V. Chernushevich, K. G. Standing and H. F. Gaertner, Bioconjugate Chem., 1996, 7, 552-556.

649 H. F. Gaertner and R. E. Offord, Bioconjugate Chem., 1996, 7, 38-44.

650 E. H. Nardin, J. M. Calvo-Calle, G. A. Oliveira, P. Clavijo, R. Nussenzweig, R. Simon, W. Zeng and K. Rose, Vaccine, 1998, 16, 590-600.

651 D. Chelius and T. A. Shaler, Bioconjugate Chem., 2003, 14, 205-211.

652 Y. Zeng, T. N. C. Ramya, A. Dirksen, P. E. Dawson and J. C. Paulson, Nat. Methods, 2009, 6, 207-209.

653 H. B. Dixon, Biochem. J., 1968, 107, 124.

654 O. El Mahdi and O. Melnyk, Bioconjugate Chem., 2013, 24, 735-765. 
655 G. L. Thomas and R. J. Payne, Chem. Commun., 2009, 4260-4262.

656 J. Chen, P. Wang, J. Zhu, Q. Wan and S. J. Danishefsky, Tetrahedron, 2010, 66, 2277-2283.

657 R. Okamoto and Y. Kajihara, Angew. Chem., Int. Ed., 2008, 47, 5402-5406.

658 R. Kaiser and L. Metzka, Anal. Biochem., 1999, 266, 1-8.

659 R. B. Merrifield, J. Am. Chem. Soc., 1963, 85, 2149-2154.

660 S. Chattopadhaya, R. Srinivasan, D. S. Y. Yeo, G. Y. J. Chen and S. Q. Yao, Bioorg. Med. Chem., 2009, 17, 981-989.

661 J. Xiao, A. Burn and T. J. Tolbert, Bioconjugate Chem., 2008, 19, 1113-1118.

662 D. P. Nguyen, T. Elliott, M. Holt, T. W. Muir and J. W. Chin, J. Am. Chem. Soc., 2011, 133, 11418-11421.

663 M. Schnolzer and S. Kent, Science, 1992, 256, 221.

664 P. Dawson, T. Muir, I. Clark-Lewis and S. Kent, Science, 1994, 266, 776.

665 T. Wieland, E. Bokelmann, L. Bauer, H. U. Lang and H. Lau, Justus Liebigs Ann. Chem., 1953, 583, 129-149.

666 T. M. Hackeng, J. H. Griffin and P. E. Dawson, Proc. Natl. Acad. Sci. U. S. A., 1999, 96, 10068-10073.

667 E. C. B. Johnson and S. B. H. Kent, J. Am. Chem. Soc., 2006, 128, 6640-6646.

668 T. Durek and P. F. Alewood, Angew. Chem., Int. Ed., 2011, 50, 12042-12045.

669 B. Dang, T. Kubota, K. Mandal, F. Bezanilla and S. B. H. Kent, J. Am. Chem. Soc., 2013, 135, 11911-11919.

670 P. E. Lobban and A. D. Kaiser, J. Mol. Biol., 1973, 78, 453-471.

671 D. A. Jackson, R. H. Symons and P. Berg, Proc. Natl. Acad. Sci. U. S. A., 1972, 69, 2904-2909.

672 Y. Sohma, B. L. Pentelute, J. Whittaker, Q.-X. Hua, L. J. Whittaker, M. A. Weiss and S. B. H. Kent, Angew. Chem., Int. Ed., 2008, 47, 1102-1106.

673 L. Raibaut, N. Ollivier and O. Melnyk, Chem. Soc. Rev., 2012, 41, 7001-7015.

674 Z. Harpaz, P. Siman, K. S. A. Kumar and A. Brik, ChemBioChem, 2010, 11, 1232-1235.

675 D. Bang, B. L. Pentelute and S. B. H. Kent, Angew. Chem., Int. Ed., 2006, 45, 3985-3988.

676 D. Macmillan, Angew. Chem., Int. Ed., 2006, 45, 7668-7672.

677 Y. Yuan, J. Chen, Q. Wan, Z. Tan, G. Chen, C. Kan and S. J. Danishefsky, J. Am. Chem. Soc., 2009, 131, 5432-5437.

678 W. R. Gordon, D. Bang, W. D. Hoff and S. B. Kent, Bioorg. Med. Chem., 2013, 21, 3436-3442.

679 G. I. Tesser and I. C. Balvert-Geers, Int. J. Pept. Protein Res., 1975, 7, 295-305.

680 A. D. Gorse and J. E. Gready, Protein Eng., 1997, 10, 23-30. 681 J. A. Camarero, G. J. Cotton, A. Adeva and T. W. Muir, J. Pept. Res., 2009, 51, 303-316.

682 D. Veber, J. Milkowski, S. Varga, R. Denkewalter and R. Hirschmann, J. Am. Chem. Soc., 1972, 94, 5456-5461.

683 D. Bang and S. Kent, Angew. Chem., Int. Ed., 2004, 43, 2534-2538.

684 S. Lahiri, M. Brehs, D. Olschewski and C. F. W. Becker, Angew. Chem., Int. Ed., 2011, 50, 3988-3992.
685 S. Ueda, M. Fujita, H. Tamamura, N. Fujii and A. Otaka, ChemBioChem, 2005, 6, 1983-1986.

686 P. Botti, M. Villain, S. Manganiello and H. Gaertner, Org. Lett., 2004, 6, 4861-4864.

687 J.-S. Zheng, H.-K. Cui, G.-M. Fang, W.-X. Xi and L. Liu, ChemBioChem, 2010, 11, 511-515.

688 R. Ingenito, E. Bianchi, D. Fattori and A. Pessi, J. Am. Chem. Soc., 1999, 121, 11369-11374.

689 S. Aimoto, Biopolymers, 1999, 51, 247-265.

690 M. Schnölzer, P. Alewood, A. Jones and D. Alewood, Int. J. Pept. Protein Res., 2007, 13, 31-44.

691 R. von Eggelkraut-Gottanka, A. Klose, A. G. Beck-Sickinger and M. Beyermann, Tetrahedron Lett., 2003, 44, 3551-3554.

692 J. B. Blanco-Canosa and P. E. Dawson, Angew. Chem., Int. Ed., 2008, 47, 6851-6855.

693 F. Thomas, J. Pept. Sci., 2013, 19, 141-147.

694 T. W. Muir, D. Sondhi and P. A. Cole, Proc. Natl. Acad. Sci. U. S. A., 1998, 95, 6705-6710.

695 R. David, M. P. O. Richter and A. G. Beck-Sickinger, Eur. J. Biochem., 2004, 271, 663-677.

696 R. R. Flavell and T. W. Muir, Acc. Chem. Res., 2009, 42, 107-116.

697 J. D. Warren, J. S. Miller, S. J. Keding and S. J. Danishefsky, J. Am. Chem. Soc., 2004, 126, 6576-6578.

698 K. Sato, A. Shigenaga, K. Tsuji, S. Tsuda, Y. Sumikawa, K. Sakamoto and A. Otaka, ChemBioChem, 2011, 12, 1840-1844.

699 Y. Ohta, S. Itoh, A. Shigenaga, S. Shintaku, N. Fujii and A. Otaka, Org. Lett., 2006, 8, 467-470.

700 A. Romanelli, A. Shekhtman, D. Cowburn and T. W. Muir, Proc. Natl. Acad. Sci. U. S. A., 2004, 101, 6397-6402.

701 F. Nagaike, Y. Onuma, C. Kanazawa, H. Hojo, A. Ueki, Y. Nakahara and Y. Nakahara, Org. Lett., 2006, 8, 4465-4468.

702 H. Hojo, Y. Onuma, Y. Akimoto, Y. Nakahara and Y. Nakahara, Tetrahedron Lett., 2007, 48, 25-28.

703 A. Otaka, K. Sato, H. Ding and A. Shigenaga, Chem. Rec., 2012, 12, 479-490.

704 J. Kang and D. Macmillan, Org. Biomol. Chem., 2010, 8, 1993-2002.

705 L. A. Erlich, K. S. A. Kumar, M. Haj-Yahya, P. E. Dawson and A. Brik, Org. Biomol. Chem., 2010, 8, 2392-2396.

706 K. Sato, A. Shigenaga, K. Kitakaze, K. Sakamoto, D. Tsuji, K. Itoh and A. Otaka, Angew. Chem., Int. Ed., 2013, 52, 7855-7859.

707 N. Ollivier, J. Dheur, R. Mhidia, A. Blanpain and O. Melnyk, Org. Lett., 2010, 12, 5238-5241.

708 J. Dheur, N. Ollivier, A. Vallin and O. Melnyk, J. Org. Chem., 2011, 76, 3194-3202.

709 W. Hou, X. Zhang, F. Li and C.-F. Liu, Org. Lett., 2011, 13, 386-389.

710 N. Ollivier, L. Raibaut, A. Blanpain, R. Desmet, J. Dheur, R. Mhidia, E. Boll, H. Drobecq, S. L. Pira and O. Melnyk, J. Pept. Sci., 2013, 20, 92-97.

711 N. Ollivier, J. Vicogne, A. Vallin, H. Drobecq, R. Desmet, O. El Mahdi, B. Leclercq, G. Goormachtigh, V. Fafeur and O. Melnyk, Angew. Chem., Int. Ed., 2011, 51, 209-213. 
712 L. Yan and P. Dawson, J. Am. Chem. Soc., 2001, 123, 526-533.

713 D. Crich and A. Banerjee, J. Am. Chem. Soc., 2007, 129, 10064-10065.

714 P. Botti and S. Tchertchian, WO2006133962, 2006, https:// patentscope.wipo.int/search/en/detail.jsf?docId=WO2006 133962.

715 L. E. Canne, S. J. Bark and S. B. Kent, J. Am. Chem. Soc., 1996, 118, 5891-5896.

716 C. Haase, H. Rohde and O. Seitz, Angew. Chem., Int. Ed., 2008, 47, 6807-6810.

717 J. Chen, Q. Wan, Y. Yuan, J. Zhu and S. J. Danishefsky, Angew. Chem., Int. Ed., 2008, 47, 8521-8524.

718 Z. Tan, S. Shang and S. J. Danishefsky, Angew. Chem., Int. Ed., 2010, 49, 9500-9503.

719 S. Shang, Z. Tan, S. Dong and S. J. Danishefsky, J. Am. Chem. Soc., 2011, 133, 10784-10786.

720 S. D. Townsend, Z. Tan, S. Dong, S. Shang, J. A. Brailsford and S. J. Danishefsky, J. Am. Chem. Soc., 2012, 134, 3912-3916.

721 H. Ding, A. Shigenaga, K. Sato, K. Morishita and A. Otaka, Org. Lett., 2011, 13, 5588-5591.

722 R. E. Thompson, B. Chan, L. Radom, K. A. Jolliffe and R. J. Payne, Angew. Chem., Int. Ed., 2013, 52, 9723-9727.

723 P. Siman, S. V. Karthikeyan and A. Brik, Org. Lett., 2012, 14, 1520-1523.

724 J. P. Tam and Q. Yu, Biopolymers, 1998, 46, 319-327.

725 C. Dose and O. Seitz, Org. Biomol. Chem., 2004, 2, 59-65.

726 L. R. Malins, K. M. Cergol and R. J. Payne, ChemBioChem, 2013, 14, 559-563.

727 K. K. Pasunooti, R. Yang, S. Vedachalam, B. K. Gorityala, C.-F. Liu and X.-W. Liu, Bioorg. Med. Chem. Lett., 2009, 19, 6268-6271.

728 R. Yang, K. K. Pasunooti, F. Li, X.-W. Liu and C.-F. Liu, J. Am. Chem. Soc., 2009, 131, 13592-13593.

729 Y.-Y. Yang, S. Ficht, A. Brik and C.-H. Wong, J. Am. Chem. Soc., 2007, 129, 7690-7701.

730 Q. Wan and S. J. Danishefsky, Angew. Chem., Int. Ed., 2007, 119, 9408-9412.

731 C. Haase and O. Seitz, Angew. Chem., Int. Ed., 2008, 47, 1553-1556.

732 P. Siman, O. Blatt, T. Moyal, T. Danieli, M. Lebendiker, H. A. Lashuel, A. Friedler and A. Brik, ChemBioChem, 2011, 12, 1097-1104.

733 T. Moyal, H. P. Hemantha, P. Siman, M. Refua and A. Brik, Chem. Sci., 2013, 4, 2496-2501.

734 B. L. Pentelute and S. B. Kent, Org. Lett., 2007, 9, 687-690.

735 N. Metanis, E. Keinan and P. E. Dawson, Angew. Chem., Int. Ed., 2010, 49, 7049-7053.

736 R. J. Hondal, B. L. Nilsson and R. T. Raines, J. Am. Chem. Soc., 2001, 123, 5140-5141.

737 M. D. Gieselman, L. Xie and W. A. van der Donk, Org. Lett., 2001, 3, 1331-1334.

738 R. Quaderer, A. Sewing and D. Hilvert, Helv. Chim. Acta., 2001, 84, 1197-1206.

739 N. A. McGrath and R. T. Raines, Acc. Chem. Res., 2011, 44, 752-761.
740 S. N. Gorlatov and T. C. Stadtman, Proc. Natl. Acad. Sci. U. S. A., 1998, 95, 8520-8525.

741 N. Metanis, E. Keinan and P. E. Dawson, J. Am. Chem. Soc., 2006, 128, 16684-16691.

742 P. E. Dawson, Isr. J. Chem., 2011, 51, 862-867.

743 Z. Wang, T. Rejtar, Z. S. Zhou and B. L. Karger, Rapid Commun. Mass Spectrom., 2010, 24, 267-275.

744 L. R. Malins and R. J. Payne, Org. Lett., 2012, 14, 3142-3145.

745 A. L. Adams and D. Macmillan, J. Pept. Sci., 2013, 19, 65-73.

746 A. Ghassemian, X. Vila-Farrés, P. F. Alewood and T. Durek, Bioorg. Med. Chem., 2013, 21, 3473-3478.

747 D. M. Coltart, Tetrahedron, 2000, 56, 3449-3491.

748 G.-M. Fang, H.-K. Cui, J.-S. Zheng and L. Liu, ChemBioChem, 2010, 11, 1061-1065.

749 J.-C. M. Monbaliu and A. R. Katritzky, Chem. Commun., 2012, 48, 11601-11622.

750 D. S. Kemp, S.-L. Leung and D. J. Kerkman, Tetrahedron Lett., 1981, 22, 181-184.

751 D. S. Kemp and D. J. Kerkman, Tetrahedron Lett., 1981, 22, 185-186.

752 D. S. Kemp, D. J. Kerkman, S.-L. Leung and G. Hanson, J. Org. Chem., 1981, 46, 490-498.

753 D. S. Kemp and N. G. Galakatos, J. Org. Chem., 1986, 51, 1821-1829.

754 D. S. Kemp, N. G. Galakatos, B. Bowen and K. Tan, J. Org. Chem., 1986, 51, 1829-1838.

755 D. S. Kemp, N. G. Galakatos, S. Dranginis, C. Ashton, N. Fotouhi and T. P. Curran, J. Org. Chem., 1986, 51, 3320-3324.

756 D. S. Kemp and R. I. Carey, J. Org. Chem., 1989, 54, 3640-3646.

757 D. S. Kemp and D. R. Buckler, Tetrahedron Lett., 1991, 32, 3009-3012.

758 D. S. Kemp and D. R. Buckler, Tetrahedron Lett., 1991, 32, 3013-3016.

759 C.-F. Liu, C. Rao and J. P. Tam, Tetrahedron Lett., 1996, 37, 933-936.

760 X.-H. Tan, X. Zhang, R. Yang and C.-F. Liu, ChemBioChem, 2008, 9, 1052-1056.

761 X. Zhang, F. Li and C.-F. Liu, Chem. Commun., 2011, 47, 1746-1748.

762 A. Dirksen and P. E. Dawson, Curr. Opin. Chem. Biol., 2008, 12, 760-766.

763 G. Zou, H. Ochiai, W. Huang, Q. Yang, C. Li and L.-X. Wang, J. Am. Chem. Soc., 2011, 133, 18975-18991.

764 S. Ratner and H. T. Clarke, J. Am. Chem. Soc., 1937, 59, 200-206.

765 C. Rao and J. P. Tam, J. Am. Chem. Soc., 1994, 116, 6975-6976.

766 P. Botti, T. D. Pallin and J. P. Tam, J. Am. Chem. Soc., 1996, 118, 10018-10024.

767 J. Shao and J. P. Tam, J. Am. Chem. Soc., 1995, 117, 3893-3899.

768 T. Wöhr, F. Wahl, A. Nefzi, B. Rohwedder, T. Sato, X. Sun and M. Mutter, J. Am. Chem. Soc., 1996, 118, 9218-9227. 
769 M. Villain, J. Vizzavona and K. Rose, Chem. Biol., 2001, 8, 673-679.

770 T. W. Muir, Annu. Rev. Biochem., 2003, 72, 249-289.

771 G. Casi, Curr. Opin. Struct. Biol., 2003, 13, 589-594.

772 G. Casi, N. Huguenin-Dezot, K. Zuberbühler, J. Scheuermann and D. Neri, J. Am. Chem. Soc., 2012, 134, 5887-5892.

773 G. J. L. Bernardes, M. Steiner, I. Hartmann, D. Neri and G. Casi, Nat. Protoc., 2013, 8, 2079-2089.

774 E. H. White, F. McCapra and G. F. Field, J. Am. Chem. Soc., 1963, 85, 337-343.

775 B. Bitler and W. D. McElroy, Arch. Biochem. Biophys., 1957, 72, 358-368.

776 J. Li, L. Chen, L. Du and M. Li, Chem. Soc. Rev., 2012, 42, 662-676.

777 H. Ren, F. Xiao, K. Zhan, Y.-P. Kim, H. Xie, Z. Xia and J. Rao, Angew. Chem., Int. Ed., 2009, 48, 9658-9662.

778 C. P. Ramil and Q. Lin, Chem. Commun., 2013, 49, 11007-11022.

779 G. Liang, H. Ren and J. Rao, Nat. Chem., 2010, 2, 54-60.

780 G. Liang, J. Ronald, Y. Chen, D. Ye, P. Pandit, M. L. Ma, B. Rutt and J. Rao, Angew. Chem., Int. Ed., 2011, 50, 6283-6286.

781 Y. Yuan, J. Zhang, M. Wang, B. Mei, Y. Guan and G. Liang, Anal. Chem., 2013, 85, 1280-1284.

782 J. Jeon, B. Shen, L. Xiong, Z. Miao, K. H. Lee, J. Rao and F. T. Chin, Bioconjugate Chem., 2012, 23, 1902-1908.

783 Y. Yuan and G. Liang, Org. Biomol. Chem., 2014, 12, 865-871. 784 E. Scoffone, A. Fontana and R. Rocchi, Biochem. Biophys. Res. Commun., 1966, 25, 170-174.

785 E. Scoffone, A. Fontana and R. Rocchi, Biochemistry, 1968, 7, 971-979.

786 L. R. Malins, K. M. Cergol and R. J. Payne, Chem. Sci., 2014, 5, 260-266.

787 J. Offer, Biopolymers, 2010, 94, 530-541.

788 X. Li, L. Zhang, S. E. Hall and J. P. Tam, Tetrahedron Lett., 2000, 41, 4069-4073.

789 A. Pictet and T. Spengler, Eur. J. Inorg. Chem., 1911, 2030-2036.

790 V. Popov, S. S. Panda and A. R. Katritzky, Org. Biomol. Chem., 2013, 11, 1594.

791 L. Zhang and J. Tam, Tetrahedron Lett., 1997, 1, 3-6.

792 N. Metanis, J. Beld and D. Hilvert, The Chemistry of Selenocysteine, John Wiley \& Sons, 2009, DOI: 10.1002/ 9780470682531.pat0582.

793 D. J. Craik and A. C. Conibear, J. Org. Chem., 2011, 76, 4805-4817.

794 L. R. Malins, N. J. Mitchell and R. J. Payne, J. Pept. Sci., 2013, 20, 64-77.

795 L. A. Wessjohann, A. Schneider, M. Abbas and W. Brandt, Biol. Chem., 2007, 388, 997-1006.
796 G. Casi, G. Roelfes and D. Hilvert, ChemBioChem, 2008, 9, 1623-1631.

797 S. M. Berry, M. D. Gieselman, M. J. Nilges, W. A. van der Donk and Y. Lu, J. Am. Chem. Soc., 2002, 124, 2084-2085.

798 M. Ralle, S. M. Berry, M. J. Nilges, M. D. Gieselman, W. A. van der Donk, Y. Lu and N. J. Blackburn, J. Am. Chem. Soc., 2004, 126, 7244-7256.

799 R. Quaderer and D. Hilvert, Chem. Commun., 2002, 2620-2621.

800 K. Forchhammer, J. Heider and C. Baron, Trends Biochem. Sci., 1991, 16, 463-467.

801 M. Muttenthaler and P. F. Alewood, J. Pept. Sci., 2008, 14, 1223-1239.

802 A. C. Obermeyer, J. B. Jarman and M. B. Francis, J. Am. Chem. Soc., 2014, 136, 9572-9579.

803 L. Yi, H. Sun, Y.-W. Wu, G. Triola, H. Waldmann and R. S. Goody, Angew. Chem., Int. Ed., 2010, 49, 9417-9421.

804 A. Schwarz, C. Wandrey, E. A. Bayer and M. Wilchek, Methods Enzymol., 1990, 184, 160-162.

805 A. Keppler, S. Gendreizig, T. Gronemeyer, H. Pick, H. Vogel and K. Johnsson, Nat. Biotechnol., 2002, 21, 86-89.

806 I. Chen, M. Howarth, W. Lin and A. Y. Ting, Nat. Methods, 2005, 2, 99-104.

807 R. R. Flavell, P. Kothari, M. Bar-Dagan, M. Synan, S. Vallabhajosula, J. M. Friedman, T. W. Muir and G. Ceccarini, J. Am. Chem. Soc., 2008, 130, 9106-9112.

808 J. M. Antos, G.-L. Chew, C. P. Guimaraes, N. C. Yoder, G. M. Grotenbreg, M. W.-L. Popp and H. L. Ploegh, J. Am. Chem. Soc., 2009, 131, 10800-10801.

809 S. Tsukiji and T. Nagamune, ChemBioChem, 2009, 10, 787-798.

810 N. Soh, Sensors, 2008, 8, 1004-1024.

811 A. Ojida, K. Honda, D. Shinmi, S. Kiyonaka, Y. Mori and I. Hamachi, J. Am. Chem. Soc., 2006, 128, 10452-10459.

812 C. T. Hauser and R. Y. Tsien, Proc. Natl. Acad. Sci. U. S. A., 2007, 104, 3693-3697.

813 S. R. Adams, R. E. Campbell, L. A. Gross, B. R. Martin, G. K. Walkup, Y. Yao, J. Llopis and R. Y. Tsien, J. Am. Chem. Soc., 2002, 124, 6063-6076.

814 T. Yamaguchi, M. Asanuma, S. Nakanishi, Y. Saito, M. Okazaki, K. Dodo and M. Sodeoka, Chem. Sci., 2014, 5, 1021-1029.

815 P. Burkhard, J. Stetefeld and S. V. Strelkov, Trends Cell Biol., 2001, 11, 82-88.

816 G. Grigoryan and A. E. Keating, Curr. Opin. Struct. Biol., 2008, 18, 477-483.

817 D. A. D. Parry, R. D. B. Fraser and J. M. Squire, J. Struct. Biol., 2008, 163, 258-269.

818 B. V. Popp and Z. T. Ball, Chem. Sci., 2011, 2, 690.

819 A. N. Zaykov, K. R. MacKenzie and Z. T. Ball, Chem. - Eur. J., 2009, 15, 8961-8965. 